\title{
The self-assembly and dynamics of
}

weakly multivalent, peptide-based, host-

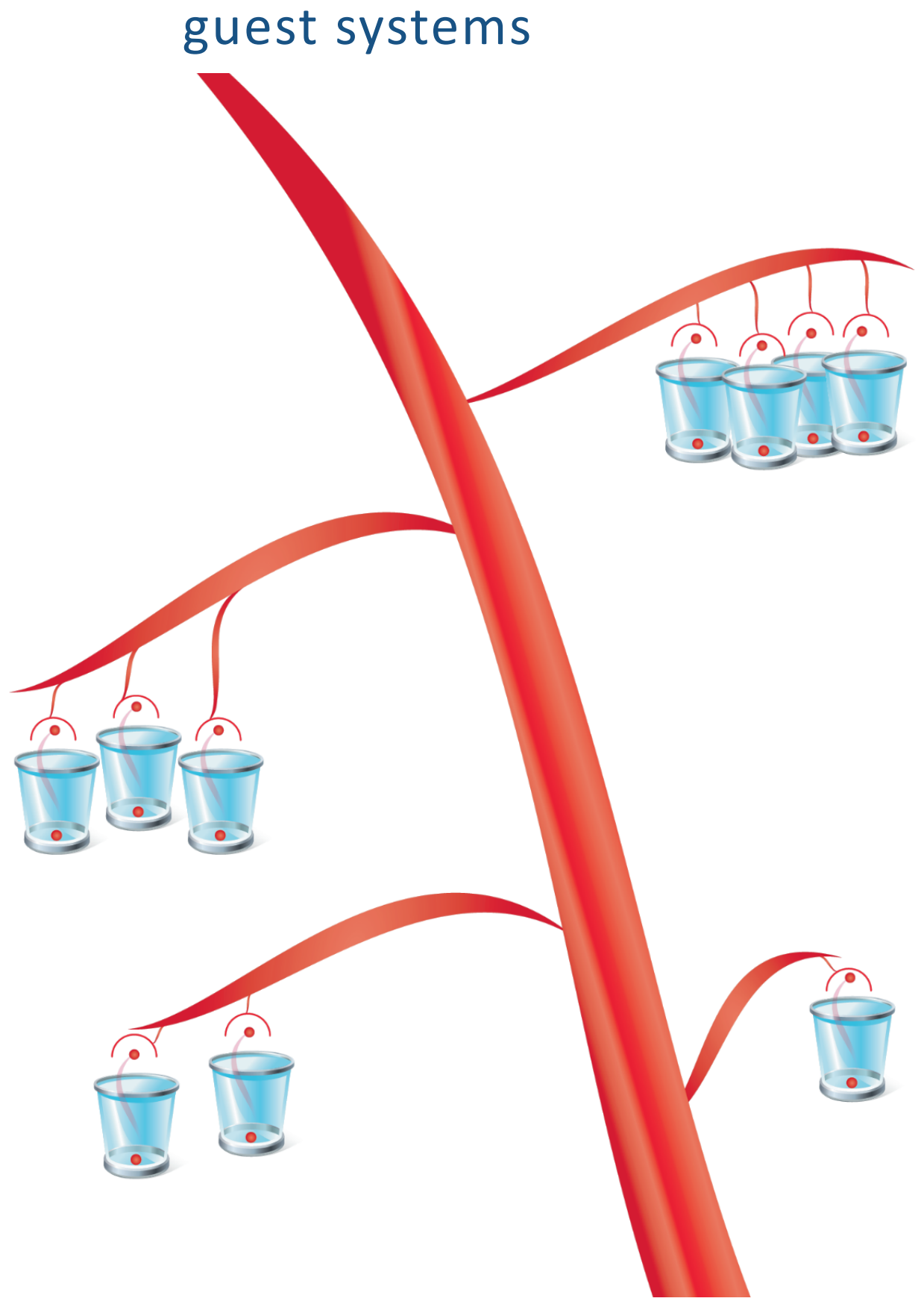

Tushar Narendra Satav 


\section{THE SELF-ASSEMBLY AND DYNAMICS OF WEAKLY MULTIVALENT, PEPTIDE-BASED, HOST-GUEST SYSTEMS}

Tushar Narendra Satav 
Members of the committee:

$\begin{array}{lll}\text { Chairman: } & \text { Prof. dr. ir. J.W.M. Hilgenkamp } & \text { (University of Twente) } \\ \text { Promotors: } & \text { Prof. dr. ir. P. Jonkheijm } & \text { (University of Twente) } \\ & \text { Prof. dr. ir. J. Huskens } & \text { (University of Twente) } \\ \text { Members: } & \text { dr. D. Thompson } & \text { (University of Limerick) } \\ & \text { Prof. dr. B. J. Ravoo } & \text { (University of Münster) } \\ \text { dr. ir. T.F.A. de Greef } & \text { (Eindhoven University of } \\ & \text { Prof. dr. M. Karperien } & \text { Technology) } \\ & \text { Prof. dr. J.J.L.M. Cornelissen } & \text { (University of Twente) } \\ \end{array}$

The research described in this thesis was performed within the laboratories of the Molecular Nanofabrication (MnF) group, the MESA+ institute for Nanotechnology, and the Department of Science and Technology (TNW) of the University of Twente. This research was supported by the European Research Council through Starting Grant Sumoman (259183).
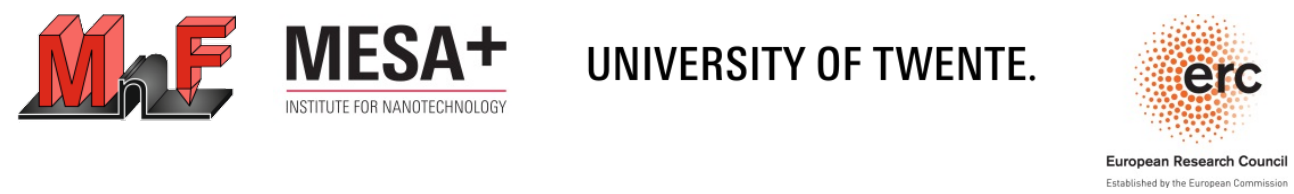

The self-assembly and dynamics of weakly multivalent, peptide-based, host-guest systems Copyright (c) 2015, Tushar Narendra Satav, Enschede, The Netherlands.

All rights reserved. No part of this thesis may be reproduced or transmitted in any form, by any means, electronic or mechanical without prior written permission of the author.

ISBN: $\quad 978-90-365-3955-5$

DOI: $\quad$ 10.3990/1.9789036539555

Cover art: Chaitanya Ghate, Tushar Satav

Printed by: Gildeprint Drukkerijen - The Netherlands

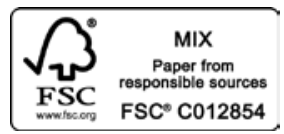




\title{
THE SELF-ASSEMBLY AND DYNAMICS OF WEAKLY MULTIVALENT, PEPTIDE- BASED, HOST-GUEST SYSTEMS
}

\author{
DISSERTATION \\ to obtain \\ the degree of doctor at the University of Twente, \\ on the authority of the rector magnificus \\ Prof. dr. H. Brinksma, \\ on account of the decision of the graduation committee, \\ to be publicly defended \\ on Thursday October 29, 2015 at 12.45 h
}

by

Tushar Narendra Satav

Born on March 24, 1987

in Amravati, India 
This dissertation has been approved by:

Promotors: $\quad$ Prof. dr. ir. P. Jonkheijm

Prof. dr. ir. J. Huskens 
"Nothing has such power to broaden the mind as the ability to investigate systematically and truly all that comes under thy observation in life."

Marcus Aurelius

This thesis is dedicated to my mom. 



\section{Table of contents}

Chapter 1: General introduction

1.1 References

Chapter 2: Effects of variations in ligand density on cell signaling

2.1 Introduction

$2.2 \quad$ Surfaces with stochastic display of ligands 8

2.2.1 Ligand density variations in monolayers 8

2.2.2 Ligand density in bio-sensing 15

2.2.3 Activating the function of growth factor by 16

2.2.4 Ligand density variations in hydrogels 20

2.2.5 Ligand density variations on peptide amphiphiles 23

2.2.6 Ligand density variations in peptide nucleic acids 24

$2.3 \quad$ Surfaces with uniform arrangement of ligands 28

2.3.1 Ligand density variations on nanocorals 28

2.3.2 Spatial clustering of ligands 29

2.4 Conclusions and future perspectives $\quad 30$

$2.5 \quad$ References 31

Chapter 3: Employing the weakly binding aromatic amino acids for multivalent peptide complexation with $\beta$-CD printboards

3.1 Introduction

3.2 Results and discussion $\quad 41$

3.2.1 Synthesis of guest peptides and their binding to $\beta$ - 43 CD SAMs

3.2.2 Linear free energy relationship 47

3.2.3 Multivalency binding model 48 
3.2.4 Influence of molecular geometry on binding 51 affinity

$\begin{array}{lll}3.3 & \text { Conclusions } & 54\end{array}$

3.4 Acknowledgments $\quad 54$

3.5 Materials and methods $\quad 55$

$\begin{array}{lll}3.6 & \text { References } & 58\end{array}$

Chapter 4: Dual functional EYPD peptide conjugates

61

facilitate binding to $\beta-C D$ surfaces and $\alpha_{9} \beta_{1}$ integrins

$\begin{array}{lll}4.1 & \text { Introduction } & 62\end{array}$

4.2 Results and discussion $\quad 63$

4.2.1 Contribution from phenylalanine to the binding of 64 a bioactive peptide to $\beta$-CD surface

4.2.2 Peptide dendrimer conjugates 69

$\begin{array}{lll}4.3 & \text { Conclusions } & \mathbf{7 6}\end{array}$

$\begin{array}{lll}4.4 & \text { Acknowledgments } & 77\end{array}$

$\begin{array}{lll}4.5 & \text { Materials and methods } & 77\end{array}$

$\begin{array}{lll}4.6 & \text { References } & 84\end{array}$

Chapter 5: Peptide-functionalized dendrimers on $\beta$-CD 87 substrates as platforms to trigger VEGF signaling

$\begin{array}{lll}5.1 & \text { Introduction } & \mathbf{8 8}\end{array}$

$\begin{array}{lll}5.2 & \text { Results and discussion } & 89\end{array}$

5.2.1 Preorganization of VEGF: A bio-signaling 89 complex on a $\beta$-CD SAM

5.2.2 Effect of variation in exposed peptide density on 90 integrin clustering

5.2.3 Effect of exposed peptide density on the 92 morphology of human dermal microvascular endothelial cells (HMVECs)

5.2.4 Effect of variation in exposed peptide density on 94 VEGF-A signaling

5.2.5 VEGF-mediated angiogenesis induced by 97 dendrimer-coated platforms 
5.2.6 Towards advanced biomedical application 101

$\begin{array}{lll}5.3 & \text { Conclusions } & 102\end{array}$

$\begin{array}{lll}5.4 & \text { Acknowledgments } & 103\end{array}$

$\begin{array}{lll}5.5 & \text { Materials and methods } & 103\end{array}$

$\begin{array}{lll}5.6 & \text { References } & 106\end{array}$

Chapter 6: Insights in the surface dynamics of weakly 111 multivalent systems

$\begin{array}{lll}\text { 6.1 Introduction } & 112\end{array}$

$\begin{array}{lll}\text { 6.2 } & \text { Results and discussion } & 113\end{array}$

6.2.1 Spreading of multivalent peptides in printing 113 experiments

6.2.2 Fluorescence recovery after photo bleaching 119 (FRAP) experiments

6.2.3 Theoretical considerations 121

$\begin{array}{lll}6.3 & \text { Conclusions } & 131\end{array}$

6.4 Acknowledgments 131

6.5 Materials and methods $\quad 131$

$\begin{array}{lll}6.6 & \text { References } & 136\end{array}$

Chapter 7: Modulating the nucleated self-assembly of tri- $\boldsymbol{\beta}^{3}$ peptides using cucurbit[n]urils

$\begin{array}{llr}7.1 & \text { Introduction } & 140\end{array}$

$\begin{array}{lll}7.2 & \text { Results and discussion } & 141\end{array}$

$\begin{array}{lll}7.3 & \text { Conclusions } & 152\end{array}$

$\begin{array}{lll}7.4 & \text { Acknowledgments } & 152\end{array}$

7.5 Materials and methods 152

$\begin{array}{lll}7.6 & \text { References } & 158\end{array}$

$\begin{array}{ll}\text { Summary } & 163\end{array}$

$\begin{array}{ll}\text { Samenvatting } & 165\end{array}$ 
About the author 


\section{Chapter 1}

\section{General introduction}

Self-assembly is the process by which individual components aggregate to form an ordered structure. In molecular self-assembly, the structure and properties of the monomer determine the nature and type of the assembly, ${ }^{1}$ although unraveling the intricate relationships between the monomers and the assembly is still one of the most important task of supramolecular chemistry. ${ }^{2-6}$ Self-assembled monolayers (SAMs) constitute an important segment in self-assembly research. For example, SAMs of $\beta$-CD form an attractive surface, due to the well-defined organization of $\beta$-CD molecules in the monolayers and the versatility of substrates onto which these SAMs can be fabricated. ${ }^{3,7} \beta$-CD molecules are known to form host-guest interactions with various molecules. ${ }^{8}$ This interaction can lead to a well-ordered organization of guest molecules on the $\beta$-CD monolayer which can be exploited for designing the next generation of nanomaterials. ${ }^{9}$

Depending on the nature of the interacting guest molecules, physical properties of the assemblies can be manipulated. ${ }^{3}$ For example, changing the multivalency in the molecular structure allows the manipulation of the affinity with the surface. These tunable differences in the surface affinity then lead to differences in the ligand density (surface coverage). This control over ligand density has tremendous importance in designing biomaterials for interrogating cell signaling. ${ }^{10,11}$ Various covalent chemistries have been reported to provide variation of the ligand density on material interfaces, but the use of non-covalent self-assembly of a bioactive ligand at an interface to vary its density and to control a biological response is so far understudied.

The aim of the work described in this thesis is to tune the host-guest interactions of multivalent, supramolecular systems to modulate the binding affinity and the selfassembly properties. A model system was developed to understand the effects of the valency and the intrinsic affinity of aromatic amino acids in a multivalent ligand on its overall binding affinity, the coverage, and the dynamics when interacting with the $\beta$-CD surface, in order to design interfaces with a tunable ligand density and dynamic 
properties for potential biomedical applications. Moreover, cucurbit[n]uril macrocycles with different cavity sizes were used to modulate the assembly of chiral $\beta$-peptides.

Chapter 2 provides an overview of the literature available on the concept of ligand density variation in biomaterial design and its implication on the cellular bio-signaling pathways. Strategies to vary the density of bioactive ligands on various materials ranging from SAMs on gold substrates (2D) to hydrogels (3D) are discussed, with a focus on their impact on cellular signaling on the nanoscale.

The effect of the number of aromatic amino acids in multivalent peptides on the binding affinity with host $\beta$-CD SAMs is studied in Chapters 3 and 4. The observed dependence of the overall binding affinity on the valency has been modelled to establish a physicalmathematical correlation between the valency of interaction, the intrinsic binding affinity, and the overall binding affinity. The influence of the weaker binding phenylalanine compared to the stronger binding tyrosine on the binding affinity of the multivalent peptide has been investigated. Bioactive peptides containing these two guest amino acids (tyrosine and phenylalanine) have been conjugated to PAMAM dendrimers of various sizes. The assembly behavior of these globular peptide dendrimer conjugates on $\beta$-CD SAMs has been investigated (Chapter 4). From the binding affinity of these dendrimers their valency of interaction with the $\beta-C D$ surface is quantified. This number is then used to quantify the peptide density on the $\beta$-CD surface available for inducing a biological response. These surfaces, with an exposed ligand density controlled by variation of the type and generation of dendrimer, are then used to study the effect of ligand density variation on the biological response in Chapter 5.

The effect of the position of the aromatic amino acids in a multivalent ligand on its surface motion is investigated in Chapter 6. Three peptides, which differed in the numbers of phenylalanine and tyrosine units as well as their position in the peptide, have been synthesized such that their overall binding affinities with the $\beta$-CD surface are similar. Surface diffusion of these peptides in the presence of various concentration of $\beta-C D$ in solution has been investigated. Different mechanisms involved in surface diffusion and their dependence on the positioning of the guest moieties in the multivalent peptides have been studied.

The influence of cucurbit[n]urils on the self-assembly of a $\beta$-peptide into protofibrils and mature fibers has been investigated in Chapter 7. The modulation of the assembly process through interactions between the cucurbit[n]urils $(\mathrm{CB}[\mathrm{n}] \mathrm{s})$ and $\beta$-tyrosines in a 
tri- $\beta^{3}$-peptide is dependent on the size of $\mathrm{CB}[\mathrm{n}]$. The observed differences in selfassembly of the peptide between $\mathrm{CB}[7]$ and the larger $\mathrm{CB}[8]$ are correlated to their difference in cavity size and the corresponding host-guest behavior. The temperature effects of the self-assembly processes are addressed as well.

\subsection{References}

1. Whitesides, G.M. \& Boncheva, M. Beyond molecules: Self-assembly of mesoscopic and macroscopic components. Proceedings of the National Academy of Sciences 99, 4769-4774 (2002).

2. Korevaar, P.A. et al. Pathway complexity in supramolecular polymerization. Nature 481, 492-496 (2012).

3. Huskens, J. et al. A model for describing the thermodynamics of multivalent host-guest interactions at interfaces. Journal of the American Chemical Society 126, 6784-6797 (2004).

4. Perl, A. et al. Gradient-driven motion of multivalent ligand molecules along a surface functionalized with multiple receptors. Nature Chemistry 3, 317-322 (2011).

5. Huskens, J. Diffusion: molecular velcro in flatland. Nature Nanotechnology 9, 500-502 (2014).

6. Ma, X. \& Zhao, Y. Biomedical applications of supramolecular systems based on host-guest interactions. Chemical Reviews 115, 7794-7839 (2014).

7. Onclin, S., Mulder, A., Huskens, J., Ravoo, B.J. \& Reinhoudt, D.N. Molecular Printboards: Monolayers of $\beta$-Cyclodextrins on Silicon Oxide Surfaces. Langmuir 20, 5460-5466 (2004).

8. Rekharsky, M. \& Inoue, Y. Chiral recognition thermodynamics of $\beta$ cyclodextrin: the thermodynamic origin of enantioselectivity and the enthalpy-entropy compensation effect. Journal of the American Chemical Society 122, 4418-4435 (2000).

9. Crespo-Biel, O., Dordi, B., Reinhoudt, D.N. \& Huskens, J. Supramolecular layer-by-layer assembly: alternating adsorptions of guest- and hostfunctionalized molecules and particles using multivalent supramolecular 
interactions. Journal of the American Chemical Society 127, 7594-7600 (2005).

10. Kilian, K.A. \& Mrksich, M. Directing stem cell fate by controlling the affinity and density of ligand-receptor interactions at the biomaterials interface. Angewandte Chemie International Edition 51, 4891-4895 (2012).

11. Koepsel, J.T. et al. A chemically-defined screening platform reveals behavioral similarities between primary human mesenchymal stem cells and endothelial cells. Integrative Biology 4, 1508-1521 (2012). 


\title{
Chapter 2
}

\section{Effects of variations in ligand density on cell}

signaling

\begin{abstract}
Multiple simultaneous interactions between receptors and ligands dictates extracellular and intracellular activities of cells. Concept of multivalency is generally used to explain interaction between two or more ligands with receptors on cell surfaces. Various strategies are reported to "induce” multivalency in materials to study their effect on cell behavior. But very few strategies are reported whereby one can tune this multivalency with precise control over density and spacing of ligands to investigate their effect on bio signaling pathways elicited by cells. In this chapter we discuss few such strategies to control density and spacing and their implications on biological functions of cells.
\end{abstract}

This Chapter was published in : T. Satav, J. Huskens, P. Jonkheijm, Small 2015 DOI: $10.1002 /$ smll.201500747. 


\subsection{Introduction}

Interactions between cell receptors and their ligands are the main responsible actors as a prelude to many cell signaling cascades. They govern the communication between cells and their surrounding environment.

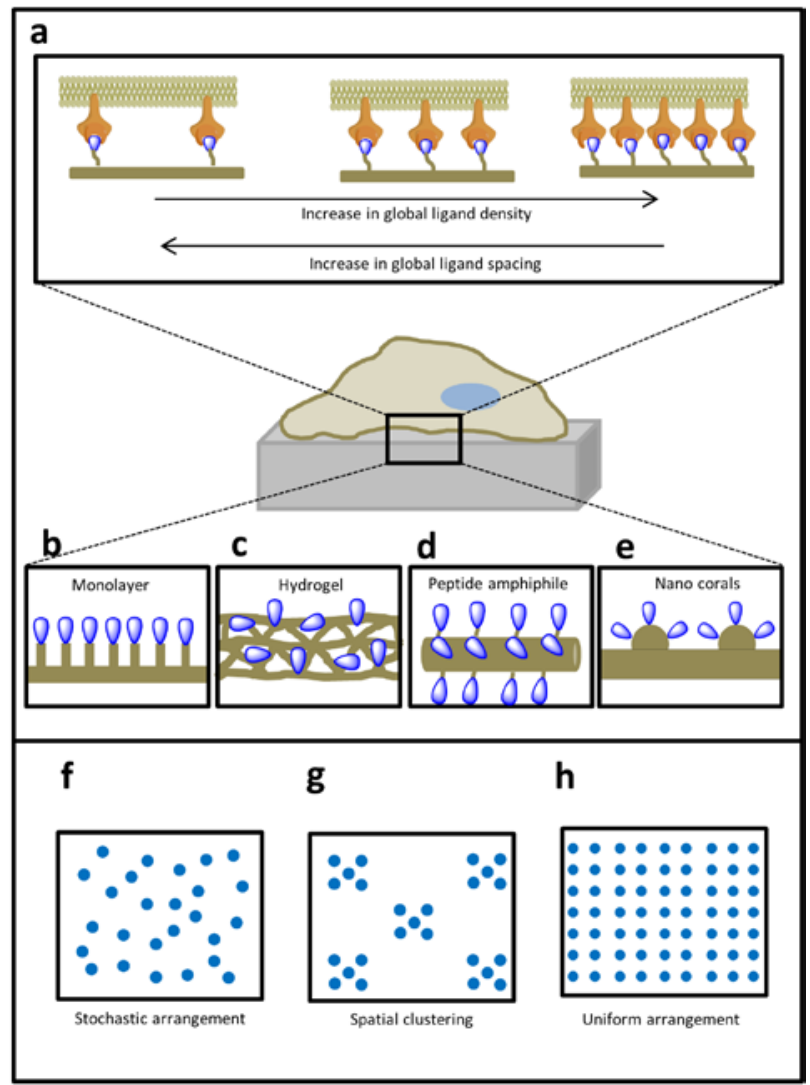

Figure 2.1 - a) Schematic illustration of various strategies to control ligand density or spacing to initiate cell signaling. b-e) Various surfaces used to control the display of ligands on surfaces. f-h) Different arrangements of ligands on surfaces (b-e) with varying global and local ligand densities.

Most of the receptors reside on the cytoplasmic membrane of cells as individual monomers but orchestrate their function as oligomeric complexes. Various examples in the literature show that when ligands are presented in multivalent formats (Figure 2.1) an increase in the selectivity of the binding with receptors is observed leading to a more 
efficient and sensitive cell signaling. ${ }^{1-4}$ This amplification of ligand receptor interactions through these multivalent formats helps to unravel features of cell signaling events which are often difficult to assess under in vivo conditions. ${ }^{5}$ For example, the cell is able to recognize adhesion ligands via interaction with transmembrane integrin proteins yielding the formation of focal adhesions through which the cytoskeleton of a cell connects to the extracellular matrix (ECM) or surfaces of biomaterials. Crucial in the maturation of these focal adhesions is the optimal spatial display of the adhesion ligands to fulfill intrinsic cell specific conditions on the geometry of cell contacts. ${ }^{6-8}$ Apart from the chemical derivatization of biomaterial surfaces to display ligands in suitable formats, other studies showed that focal adhesion formation can also be gently modulated when cells are exposed to materials that have intricate variations in physical factors such as their rigidity or nanotopographies. ${ }^{9-14}$ The importance of biomaterial design for regenerative medicine, medical devices and diagnostics is steadily increasing. At present, studies are mainly focused on changing physical properties, such as size, shape, mechanical properties, surface texture and compartmentalization in order to enhance the function of a biomaterial, once it is placed into a biological environment. However, we review and discuss that parameters like variation in ligand density and ligand spacing in biomaterials can profoundly regulate biological responses. ${ }^{15,16}$ Based on our survey of recent literature we believe that a tremendous opportunity is available for exploring methods to control the display of ligands on surfaces to modulate cell responses. ${ }^{[5]}$ In this review we will present an overview of various strategies to initiate cellular signaling as a consequence of variations in ligand densities on surfaces. Model systems that we selected comprise of self-assembled monolayers (SAMs), hydrogels, peptide amphiphiles, peptide nucleic acids and nano-corals. Although some of the model systems are challenging to translate into a clinical setting, they have yielded interesting details on the relevance of programmed ligand density for initiating cell signaling. 


\subsection{Surfaces with stochastic display of ligands}

\subsubsection{Ligand density variations on monolayers}

Self-assembled monolayers (SAMs) of alkanethiolates on gold are an excellent model system to immobilize bioactive molecules (Figure 2.1a/b, 2.2 and 2.3). ${ }^{17}$ The strategy involves mixing different ratios of ethylene glycol-terminated thiols and for example, maleimide-terminated thiols (Figure 2.3). The gold surfaces are incubated with these solutions to form uniform monolayers via the gold-thiol interaction. The maleimide group that is present in the monolayer can then be coupled to different bioactive ligands containing thiols (e.g. cysteine-containing peptides) to form surfaces with different densities of active ligands. Variations in the average ligand density can be used to relate to variations in recorded cell responses. In one report, the transmembrane integrin binding sequence Gly-Arg-Gly-Asp-Ser-Pro (GRGDSP) (Figure 2.2a/b) was presented on these SAMs in different densities and their effect on attachment, spreading, proliferation and differentiation was studied as a function of ligand density. ${ }^{12}$ To control the average ligand density in each SAM array Murphy and coworkers have mixed a bioactive GRGDSP peptide with a non-bioactive GRGESP peptide to keep the total peptide density constant at 5\% during the peptide coupling to $\mathrm{N}$-hydroxysuccinimide (NHS)-activated SAMs (Figure 2.2b). Relative quantification of the ligand density was verified by labeling the bioactive peptides with a fluorescent marker. The fluorescence intensity of each spot was found to be directly proportional to amount of active peptides included in the coupling reaction of the peptide to the surface. A clear effect of active ligand density on cell attachment and morphology was observed (Figure 2.2c). 


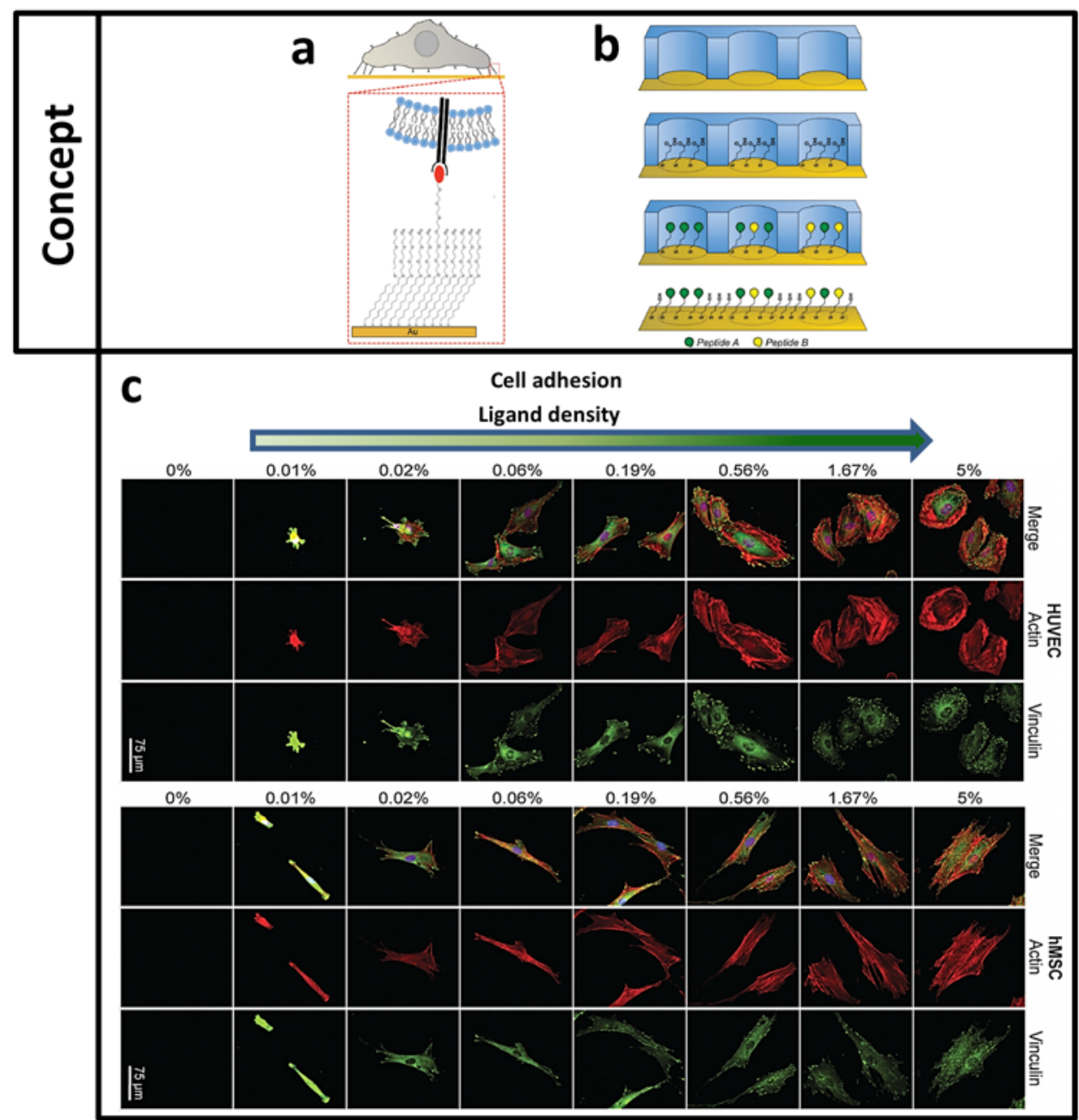

Figure 2.2 - a) SAMs can be designed to present covalently immobilized biomolecules such as the integrin binding ligand Gly-Arg-Gly-Asp-Ser-Pro (GRGDSP) which bind to cells. Effects of non-specific protein adsorption were minimized via oligo(ethylene glycol) moieties. b) Schematic representation of the fabrication of SAM arrays using an elastomeric stencil approach. c) Screening for cell morphology using SAM arrays presenting varying densities of RGD ligands (vinculin: green, actin: red, and nuclei: blue). (Reprinted with permission from Integr. Biol., 2012, 4, 1508-1521. Copyright 2012 RSC.)

An increase in cell adhesion as a function of ligand density was found to be independent on the cell types used, i.e., human umbilical vein endothelial cell (HUVECs) and human 
mesenchymal stem cells (hMSCs) (Figure 2.2c). In addition, the minimum density of GRGDSP required for observing cell attachment was also the same for both of these cell types. Both cell types were reported previously to show vinculin dependent focal adhesion formation and f-actin structure at high densities of active ligands. ${ }^{18}$ However, in this report by Murphy and coworkers in contrast, HUVECs and hMSCs showed distinct differences in focal adhesion organization at each GRGDSP density (Figure 2.2c). HUVECs showed thick f-actin stress fibers terminated by focal adhesions at the cell periphery while hMSCs showed f-actin stress fibers running longitudinally through the cells and terminated by focal adhesions at high ligand density. Upon decreasing the active ligand density HUVECs showed more sharp changes in their stress fiber formation. A more longitudinal orientation of the stress fibers and focal adhesions located at the ends of the cytoskeletal structure was observed. In the case of hMSCs the recorded changes were more subtle upon decreasing the active ligand density (Figure 2.2c). At very low active ligand density both cell types showed a compact cytoskeletal structure with highly localized f-actin and vinculin staining (Figure 2.2c). Quantification of the focal adhesion density, size and average staining intensity confirmed that the bioactive peptide density modulated the formation of focal adhesion contacts for both cell types while in the case of HUVECs a significantly higher expression of focal adhesions was observed for several GRGDSP densities in agreement with literature data. ${ }^{19-21}$ While hMSCs showed an increased level of $\beta 1$ integrin expression upon increasing the RGD density in the arrays, HUVECs lacked this response. This observation is quite remarkable as it suggests that ligand density not only influences cell adhesion and morphology but also holds promise to control biochemical signaling pathways via controlling the level of integrin expression. Changes in the active ligand density was also found to positively affect the cell proliferation ability of the platform. For both cell types cell proliferation was found to be elevated after $72 \mathrm{~h}$ upon increasing the active ligand density. While cell migration in the case of hMSCs appeared insensitive to changes in the active ligand density, HUVECs showed that only at lower active ligand density over $30 \%$ of the cells were migrating. When soluble active ligands were added to the medium the migration of the cells on the surface increased, which is presumably related to the exchange of array bound active and soluble unbound inactive ligands, which mimics a lower active ligand density array. ${ }^{13}$ In a report by Mrksich and coworkers, SAMs were exploited to investigate whether an appropriate ligand density in 
combination with an appropriated ligand affinity can lead to differential differentiation of hMSCs. ${ }^{22}$ Their SAMs were prepared by immobilizing either a linear or a cyclic variant of the cell adhesion peptide RGD to maleimide- functionalized SAMs in either high (1\%) or low $(0.1 \%)$ density (Figure $2.3 \mathrm{a})$.

It has been reported in literature that the cyclic variant of RGD has a higher binding affinity for the $\alpha_{\mathrm{v}} \beta_{3}$ integrin receptor when compared to the linear variant. Moreover, this $\alpha_{\mathrm{v}} \beta_{3}$ integrin receptor is known to be involved in adhesion and osteogenesis in mesenchymal stem cells (MSCs). ${ }^{23-25}$ Cells were then cultured on each SAM and after fixation of the cells the alkaline phosphatase (AP) expression was visualized, which is a known initial stage marker of osteogenesis (Figure 2.3c). $44 \%$ of the cells on SAMs with a high density of the high affinity (i.e. cyclic RGD) peptide showed AP expression comparable to cells that were cultured on fibronectin control SAMs (48\%). However, for cells that were cultured on SAMs with a low density of the high affinity peptide a lower expression (30\%) of AP was detected, which was higher than the AP expression level on bare SAMs (25\%). MSCs cultured on SAMs with the low affinity (i.e. linear RGD) peptide at either high or low density showed AP expression levels that were comparable to those observed on the bare SAMs. When MSCs were cultured on SAMs with control peptides (RDG) also no significant AP expression was observed indicating the specificity of the RGD-integrin interaction. These results could be validated by reverse transcriptase PCR (RT-PCR) by quantification of the AP mRNA transcript. Furthermore, MSCs cultured on the SAMs with the high affinity ligands showed a higher expression of runt-related transcription factor 2 (Runx2), which is also an indicator of cells differentiating into osteoblasts (Figure 2.3d). Together the results suggested that the SAMs with a high density of high affinity cyclic RGD peptides is most effective to promote osteogenesis. In contrast, when MSCs were cultured on SAMs with a high density of the low affinity ligand the highest expression of the skeletal muscle marker myogenic regulatory transcription factor (MyoD) was observed. When cells were stained for $\beta 3$-tubulin, which is a marker for neurogenic differentiation of MSCs, the highest expression of $\beta 3$-tubulin was observed on the SAMs with a low density of the low affinity ligands. 


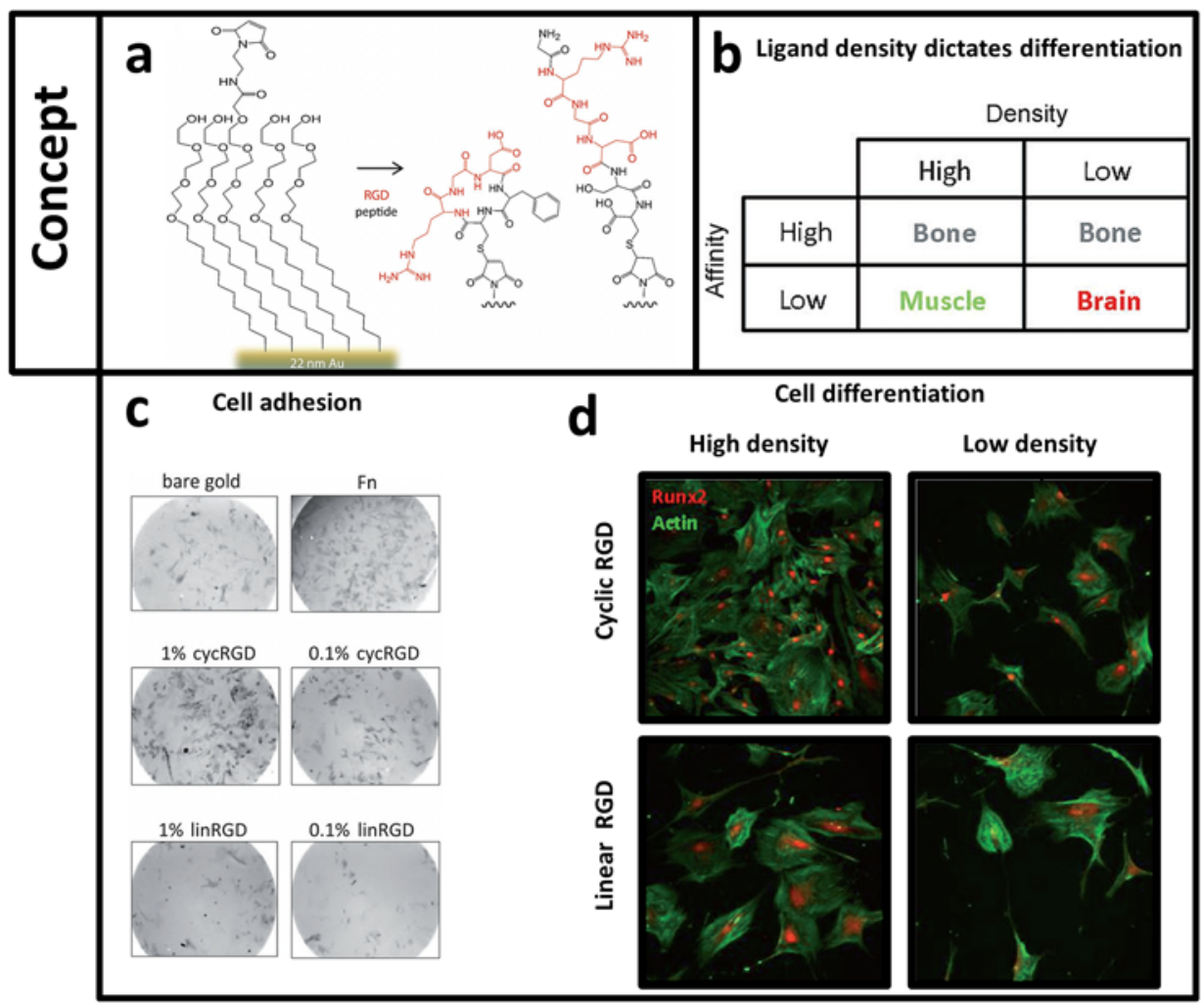

Figure 2.3 - a) Monolayers presenting adhesion ligands (RGD) were prepared on a glass slide having gold islands. Either linear or cyclic RGD ligands (shown in red) were coupled to maleimide groups in the SAM. b) Table summarizing the preferred differentiation outcomes for cells cultured on the four monolayer surfaces. c) Phase contrast images of mesenchymal stem cells stained for the osteogenesis marker, alkaline phosphatase (AP, dark gray), after 10 days of culture. d) The density and affinity of an adhesion ligand influences the differentiation of mesenchymal stem cells as analyzed using immunofluorescence imaging of markers for e.g. osteogenesis (Runx2). (Reprinted with permission from Angew. Chem. Int. Ed., 2012, 51, 48914895. Copyrights 2012 Wiley.)

In addition to neural specific marker $\beta 3$-tubulin, SAMs with a low density of the low affinity peptide also showed an elevated expression of MyoD, indicating that cells on this type of SAM show myogenic and neurogenic differentiation. Cells cultured on SAMs of high affinity ligands showed a higher focal adhesion density when compared to SAMs of low affinity ligands irrespective of ligand density, while on SAMs with a 
low density of low affinity ligands a higher expression of muscle specific myosin heavy chain (MYH) was observed. Collectively these results showed that the ligand affinity in combination with the density on the SAM clearly influences MSC differentiation (Figure 2.3b). The observations are in agreement with for example observations by others when varying the mechanical properties of the substrate onto which cells were cultured. $^{7}$ The relationship between ligand density and stem cell differentiation found in this study can inspire new design rules to modify surfaces for stem cell applications. Moreover this molecular approach of fabricating surfaces to control stem cell fate using ligand density variation technology will be immensely helpful to engineer biomaterials for stem cell therapy. For example, to enhance functional recovery after spinal cord injury, ligand density variation technology can be used to enhance neural stem cell attachment on biomaterial scaffolds. Thus ligand density variation technology might act as a powerful tool to optimize the bioefficacy of conventional bare biomedical scaffolds. $^{26}$

In the above cases SAMs were made up of alkanethiolates that are known to form monolayers with high packing density. Such high density surfaces are beyond reach when peptides were to be immobilized onto surfaces directly mainly due to conformational limits. ${ }^{27}$ To increase the peptide density in such strategies, Jiang and coworkers introduced a tetraproline linker to the bioactive ligand yielding monolayers with an increased ligand density compared to monolayers in the absence of the proline linker. Another way to increase the ligand density was achieved by introducing surface topography. 


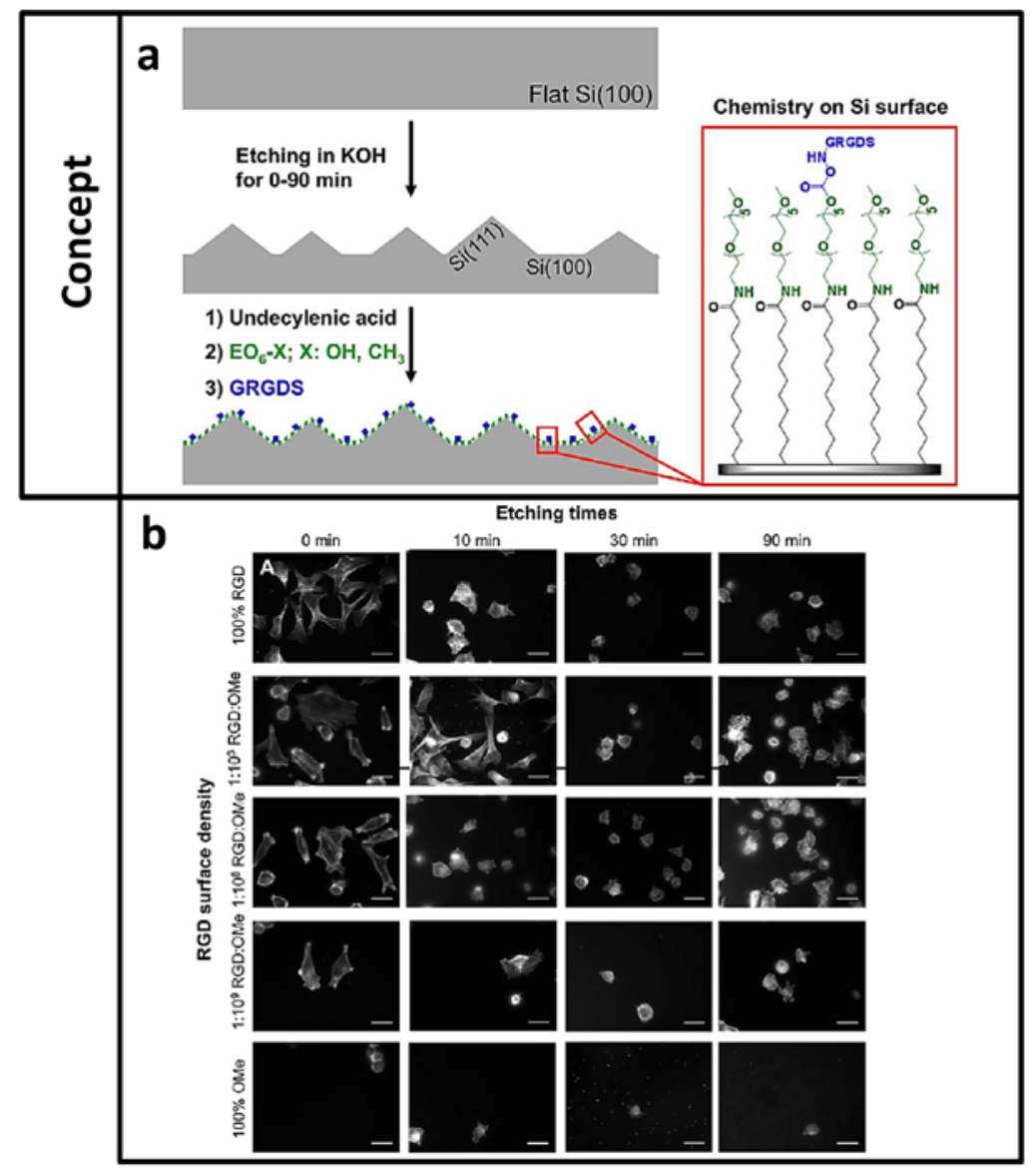

Figure 2.4 - a) Depiction of an adhesive substratum on which surface features and chemistry could be independently controlled. Etching times determine the size of pyramidal features and hence surface roughness. Subsequent functionalization with self-assembled monolayers controls RGD densities. b) Inspection of the various substrata using epifluorescence microscopy shows that serum-starved endothelial cells adhere to flat and to etched surfaces. (Reprinted from PLoS ONE, 2011, 6, 1-13.)

In a report by Gooding and coworkers, it was found that surfaces containing bioactive ligands on pyramidal topographical features showed less cell adhesion than on flat surfaces (Figure 2.4). ${ }^{28}$ However in the case of surfaces with topographical features maximum cell spreading and focal adhesion length was dependent on optimal ligand density irrespective of features and their sizes. ${ }^{29}$ These studies suggest the complex interplay between topography and variation in ligand density on endothelial cell 
behavior. These findings confirm the need for better control of these parameters in the design of materials for biomedical applications and implantable devices.

\subsubsection{Ligand density in bio-sensing}

Multivalency is known to bring selectivity in ligand-receptor interactions. This selectivity is known to be sharply dependent on a threshold ligand density. ${ }^{30}$ Below this minimum density, ligands are not able to bind to receptors while above this limit ligands can selectively bind to receptors. Ligand density-dependent bio-sensing is a viable strategy for various disease diagnostic applications. One example where such strategy has been applied was for the high-throughput sensing of cancer cells through exploiting the interaction between RGD ligands and integrins. ${ }^{31}$ Label-free resonant waveguide grating (RWG) imaging was exploited to investigate the correlation between cell spreading kinetics of cancer (HeLa) cells and the average ligand density. The ligand density on the RWG sensor surface was tuned over four orders of magnitudes by coadsorbing an biologically inactive PEG polymer with that of an RGD- functionalized polymer. HeLa cells were grown on the RWG sensors and a direct correlation was established between the maximum biosensor response and the ligand density. It was observed that the maximum biosensor response (which depended on the fraction of the sensor surface covered with spread cells) increased with an increase in active ligand density in the polymer adsorbed on the sensor surface. Saturation of the biosensor response at very high ligand density was observed, which was attributed to reaching an interligand spacing below $10 \mathrm{~nm}$, which is same as the distance between two integrin's on the cell surface. The rate of spreading was found to be independent of ligand density variations. This was attributed to the fact that the rate constant of spreading depends on the growth of filopodia governed by actin polymerization which is independent of ligand density variations. Various studies show a significant impact of the RGD ligand density on cell spreading, however most of the studies are restricted to quantifying cell adhesion and spreading at a single time point without considering the dynamic aspect of adhesion and spreading. ${ }^{11,32-34}$ This device can be applied to live cell assays where obtaining reliable and high quality kinetic data is crucial. 


\subsubsection{Activating the function of growth factor by spatially organizing binding ligands on SAMs}

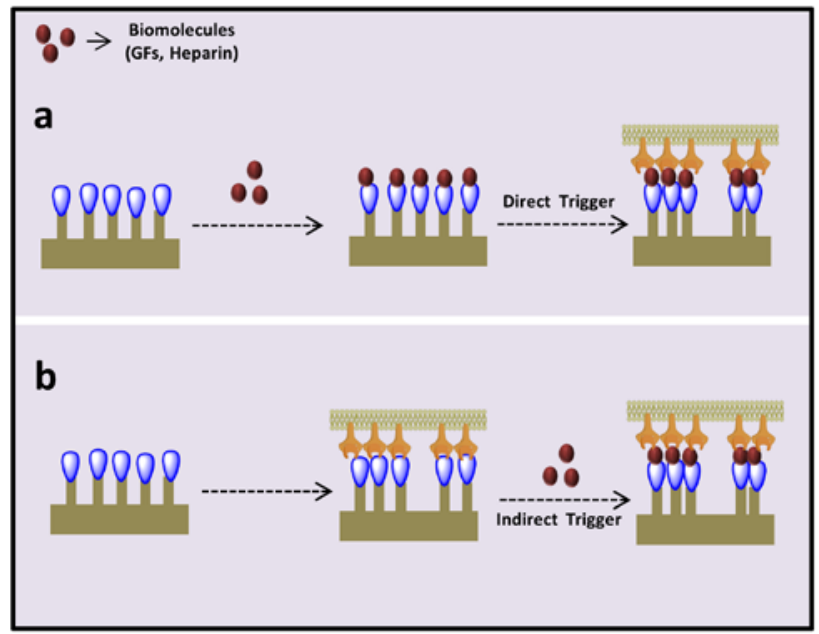

Figure 2.5 - Two types of strategies to initiate cell signaling are depicted schematically. a) Direct activation is achieved when ligands can directly interact with growth factors (GFs) to initiate a cell signaling pathway. b) Indirect activation is achieved when ligands first interact with GF receptors and this receptor ligand interaction (receptor clustering) then recruits GFs to these sites. Both strategies are influenced by surface ligand density and spacing.

Growth factor (GF) proteins are an important class of biomolecules involved in regulating cellular growth, proliferation and differentiation. ${ }^{35}$ Growth factors can interact with a specific growth factor receptor embedded within the plasma membrane surface of a cell. This interaction leads to the activation of biochemical signaling pathway. However in many cases precise spatial control is required over the activation of growth factor signaling (Figure 2.6a,b). For example, TGF- $\beta$ signaling is initiated when the growth factor binds and mediates the assembly and activation of a cell-surface receptor complex composed of sub-type I and II of TGF- $\beta$ receptors (T $\beta$ RI and T $\beta$ RII). ${ }^{36}$ TGF- $\beta$ binds tightly to T $\beta$ Rs with $K_{d}$ of $5-30 \mathrm{pM}$ and depends on avidity (Figure 6b). On the cell surface, T $\beta$ RI and T $\beta$ RII exist as noncovalent homodimers and this organization can promote TGF- $\beta$ complexation. Kiessling and coworkers devised a strategy to preorganize the TGF- $\beta$ receptor complex on surfaces by preparing SAMs 
displaying either peptide ligand LTGKNFPMFHRN (Pep1) or MHRMPSFLPTTL (Pep2) that both can interact with both T $\beta$ Rs but interfere with neither TGF- $\beta$ complexation nor signaling (Figure 2.6). ${ }^{37}$

The observation of the translocation of Smad2/3 from the cytosol to the nucleus confirms specific TGF- $\beta$ signaling (Figure 2.6c) when cells were grown on either the Pep1 or Pep2-SAMs. The Smad2/3 translocalization was inhibited by a T $\beta$ RI kinase inhibitor (SB-431542), indicating that Pep1- and Pep2-SAMs act through the TßRI kinase (Figure 2.6c). In addition, the expression levels of genes PAI-1 and Snail, which are associated to Smad3 nuclear translocation, were up-regulated in growth medium. Interestingly, the epithelial cells cultured on Pep1- or Pep2-SAMs lost polarity and adopted a mesenchymal morphology (Figure 2.6d). Peptide-functionalized SAMs also promoted the up-regulation of alpha-smooth muscle actin ( $\alpha$-SMA), which is a mesenchymal marker (Figure 2.6d). By spatially organizing ligands on surfaces, Kiessling and coworkers showed that it is possible to gain control over growth factor activity. An important advantage of this strategy is that no exogenous supply of growth factor was required. Due to clustering of cell surface receptors by ligands presented on SAMs, the threshold required for growth factor activation was decreased. Thus, growth factor present in tissue (at low levels) is sufficient to trigger the bio-signaling pathway. 


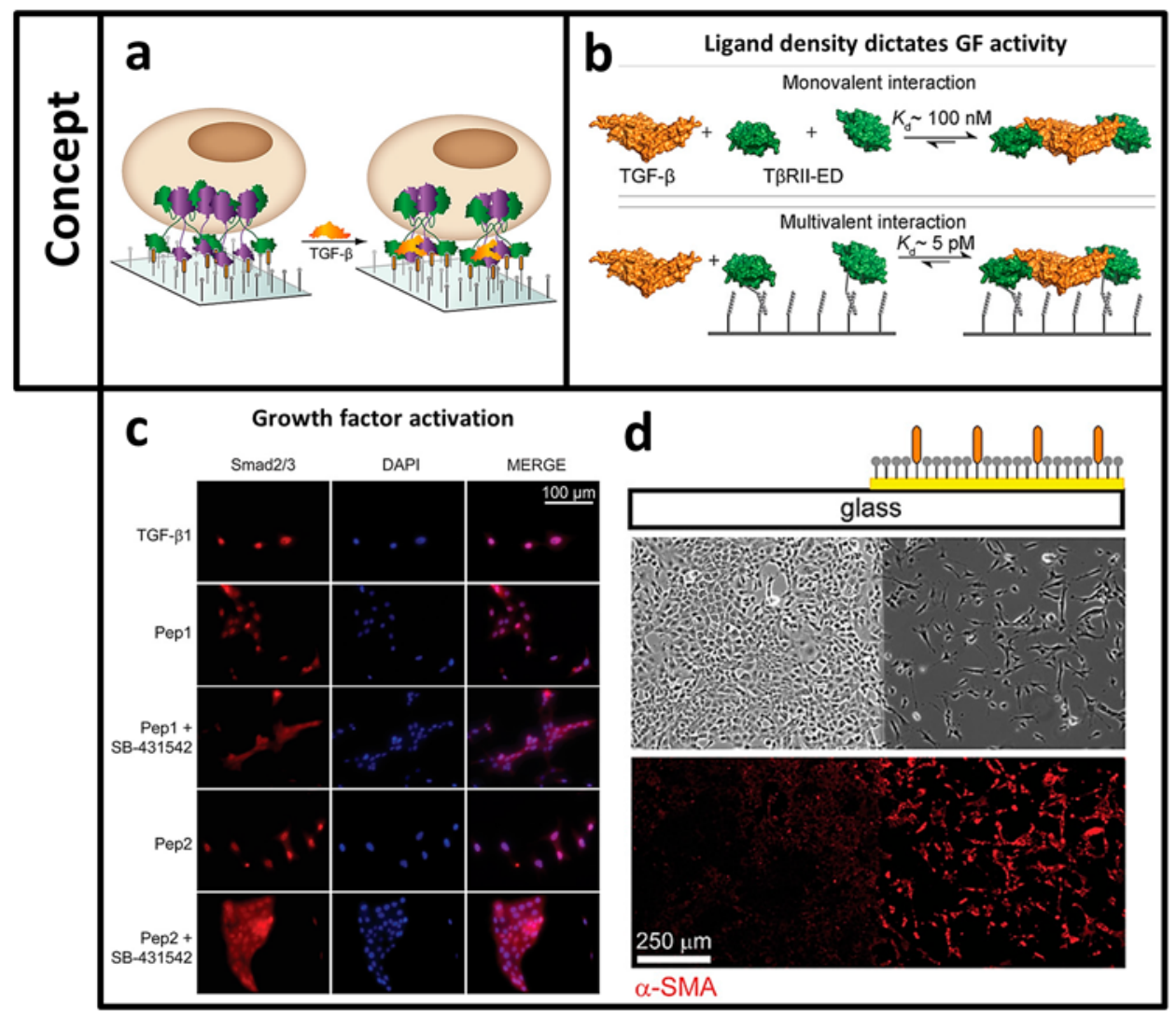

Figure 2.6 - a) The TGF- $\beta$ signaling complex is preorganized using a SAM composed of an alkanethiol displaying a T $\beta$ R-binding peptide. b) TGF- $\beta$ binds to soluble monomeric T $\beta$ RII-ED with modest affinity $\left(K_{d} \sim 100 \mathrm{nM}\right)$, but binds to immobilized T $\beta$ RII-ED with high functional affinity $\left(K_{d} \sim 5 \mathrm{pM}\right)$. c) Cells cultured for $48 \mathrm{~h}$ with TGF- $\beta$ treatment or on Pep1 and Pep2(which are specific for T $\beta \mathrm{R}-\mathrm{I}$ and T $\beta \mathrm{R}-\mathrm{II}$ extracellular domain) functionalized surfaces were stained for Smad2/3. All conditions were also evaluated in the presence of T $\beta$ R-I kinase inhibitor (SB-431542) for comparison. d) Cell fate is controlled by ligand density dependent GF activation as indicated by $\alpha$-SMA staining. (Reprinted from Proc. Natl. Acad. Sci. USA, 2011, 108, 11745 11750. Copyrights 2011 PNAS.)

An alternative strategy to modulate growth factor activity can be achieved by modulating the density of growth factor-binding ligands on surfaces. As an example, Murphy and coworkers prepared SAMs with KRTGQYKL peptide ligands that are specific for sequestering heparin that specifically binds fibroblast growth factor-2 (FGF- 
2). ${ }^{38}$ The SAMs also contained RGD adhesive ligands along with the heparin-binding ligand. The SAM with the highest density of heparin-binding ligands (2\%) showed increased cell proliferation in the presence of FBS and FGF-2 compared to SAMs with a lower density of this peptide $(<1 \%)$. This suggests that the ligand density can also be used to indirectly influence the growth factor activity. In one report by Gaus and coworkers ligand density was used to study integrin-mediated VEGF activation. ${ }^{39}$ Silicon surfaces were functionalized with 1-amino-hexa(ethylene oxide) and 1-amino hexa(ethylene oxide) monomethyl ether. The hydroxyl-terminated molecules were activated with 4-dimethyl aminopyridine (DMAP) and treated with GRGDS peptide. The RGD density was varied by changing the ratio of 1-amino-hexa(ethylene oxide) to 1-amino-hexa(ethylene oxide) monomethyl ether. The final RGD density was calculated using X-ray photoelectron spectroscopy. Average RGD spacing was calculated assuming a random distribution of RGD-functionalized glycol with the inert glycol molecule. On the RGD-modified surfaces biphasic endothelial cell adhesion was observed. Optimal cell adhesion was observed on surfaces with moderate ligand spacing. Surprisingly, surfaces with minimum RGD spacing (maximum ligand density) did not translate into increased cell adhesion or spreading. Next, the influence of RGD density on integrin activation and growth factor signaling was studied. $\alpha 5 \beta 1$ integrin activation was found highest on surfaces with moderate ligand spacing, while surfaces with lower and higher ligand spacings showed similar levels of integrin activation. A similar trend was observed for VEGF activation. Thus, nanoscale variation in ligand spacing (or density) can significantly influence the level of receptor activation which in turn leads to differences in regulation of signaling pathways in cells. These results confirm that surfaces with tailored ligand densities can be used to control the stability and activation of growth factors on these surfaces. Strategies such as these are of importance to improve treatments based on growth factor therapy and to improve the incorporation and prolonged function of implants in surrounding tissue.

Ligand density can also be used to control the long-term self-renewal and differentiation of cells for advanced cell therapies. One such report by Brandenberger and coworkers involved the use of acrylate surfaces exposing different densities of active peptide ligands. ${ }^{40}$ In this study various peptides derived from active domains of extracellular matrix proteins were conjugated to the acrylate surfaces. Human embryonic stem cells (hESC) adhered to these SAMs dependent on the ligand density. At higher 
concentrations of ligands robust cell attachment was observed, which was comparable to matrigel control surfaces.

\subsubsection{Ligand density variations in hydrogels}

Although monolayers as discussed above are ideal platforms for rapid and easy quantification of cell surface interactions, the three-dimensional (3D) environment as present under in vivo conditions is not included. Cells show a different response in a 2D compared to a 3D environment. For instance matrix metalloproteinases (MMPs) are not necessary in the formation of capillary networks on (monolayer) surfaces, however MMPs are imperative for capillary growth in a 3D environment due to restriction in cell movement. ${ }^{41-44}$ Another example is that endothelial cells in a $2 \mathrm{D}$ culture are directly exposed to soluble growth factors ${ }^{41}$ whereas in a $3 \mathrm{D}$ environment growth factors have to diffuse into the hydrogel to spatially guide vascularization. ${ }^{45-47}$ These examples and others have shown the importance of studying the effects of ligand density variation in 3D on cell behavior. In a recent study performed by Murphy and coworkers, poly(ethylene glycol)-based hydrogels were functionalized with different densities of RGD peptides (Figure 2.7a) in an array format (Figure 2.7b), and the pro-angiogenic behavior in human umbilical vein endothelial cells (HUVECs) was evaluated. ${ }^{48}$ One type of hydrogel constructs contained RGD ligands and MMP degradable peptide ligands (KCGGPQGIWGQGCK), while in another hydrogel none of these peptides were included. The RGD ligand density was varied by mixing RGD and RDG (scrambled peptide) hydrogels, while maintaining the overall peptide concentration the same (Figure 2.7c). Furthermore, the modulus of the hydrogel was tuned by changing the crosslinking density from 4.2, 5 to $7 \%$ w/v. In hydrogels having a low modulus, capillary like structure (CLS) length, which is a measure for the extent of cell adhesion, was found to be directly proportional to the adhesion peptide density in the hydrogel. In contrast, in the high modulus hydrogels, the CLS length increased only until a certain density of adhesion peptide after which no significant increase in CLS length was measured (Figure 2.7d). In hydrogels supplemented with a VEGFR-2 inhibitor the ligand dependence trend was changed (Figure 2.7e). In low modulus hydrogel CLS length increased with increase in ligand density, while in medium and high modulus hydrogel there was no influence of ligand density on CLS length. These results 
demonstrate the intricate interplay between the ligand density as well as underlying material property (modulus).

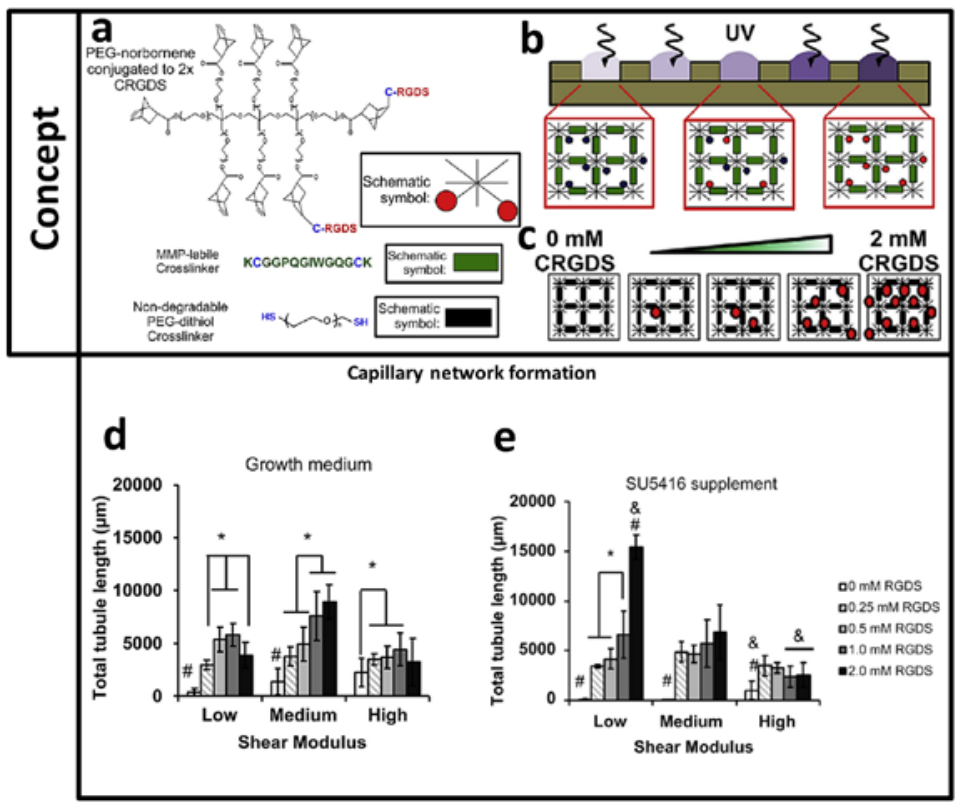

Figure 2.7 - a) Molecules included in PEG hydrogels. b) The hydrogel spots are crosslinked using UV light. c) Pictorial representation of GRGDS density change. d) Total tubule length was determined by manually measuring tubule lengths throughout the spots from epifluorescence Zstack images. The cells were stained using Cell Tracker Green and Hoechst nuclear stain $24 \mathrm{~h}$ after encapsulation. e) Tubulogenesis when VEGFR2 was inhibited by $10 \mathrm{mM}$ SU5416 supplementation. ${ }^{*} p<0.05 \& p<0.05$ compared to all equivalent CRGDS concentrations in other modulus conditions. (Reprinted with permission from Biomaterials., 2014, 45, 2149 - 2161. Copyrights 2014 Elsevier.)

In another study by Kiessling and coworkers, acrylamide-based hydrogels were immobilized on glass surfaces to form matrices, and RGD peptides were immobilized into these matrices at different densities (Figure 2.8a) to study the self-renewal of human pluripotent stem cells. ${ }^{49}$ Immobilizing the hydrogels on surfaces aids easy handling and cell analysis. Also in this study the ligand density was found to be directly proportional to cell adhesion. Moreover the results of this study showed that depending 
on the type of peptide used i.e. either integrin binding or glycosaminoglycan binding peptide, pluripotency of human embryonic stem cells was controlled.

Mesenchymal stem cells (MSCs) attracted great attention in tissue engineering and regenerative medicine. ${ }^{50,51}$ MSCs have been reported to be highly sensitive to cues from the physical environment such as elasticity ${ }^{7,52-54}$ geometry $^{55}$ and topography ${ }^{56,57}$ which affects their growth and ability to differentiate in to different lineages. Apart from these physical cues, influence of the spatial arrangement of ligands on MSC behavior was studied. $^{58}$

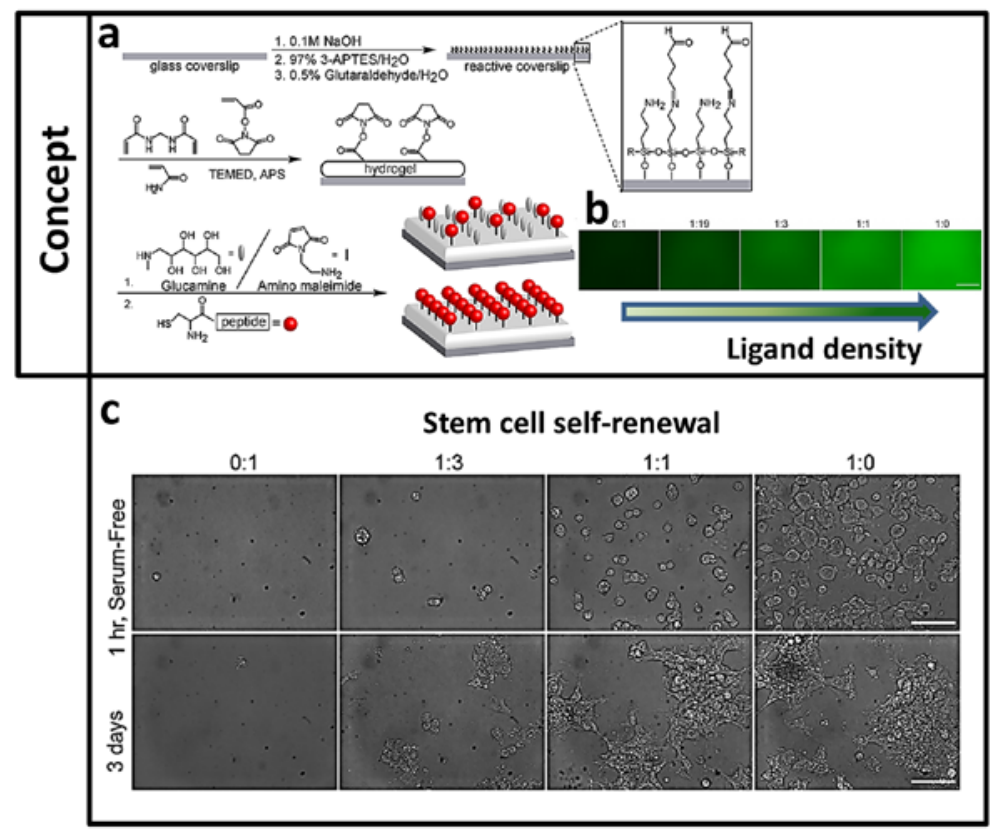

Figure 2.8- a) Production of polyacrylamide hydrogels with controlled presentation of peptides. Hydrogels were appended onto functionalized glass coverslips. Functionalization of these materials was conducted to introduce a nonbinding group (glucamine) and peptide sequences of interest. b) Fluorescence microscopy images of hydrogels functionalized with fluorescein-labeled peptide (FITC-Acp-GRGDSC). (Ratio represents the maleimide peptide:glucamine composition in hydrogel). c) Bright-field images of embryonal carcinoma cells cultured on hydrogels under serum-free conditions. Role of ligand density in cell binding (top) and results after 3 days of growth (bottom). Scale bars: b) $500 \mu \mathrm{m}$, c) $100 \mu \mathrm{m}$. (Reprinted with permission from ACS Nano, 2012, 6, 10168 - 10177. Copyrights 2012 ACS.) 
In this context, RGD surfaces with different ligand density and interligand spacing were fabricated on maleimide-functionalized polystyrene-block-poly(ethyleneoxide) copolymers. Surfaces with an average lateral spacing of 34, 44, 50 and $62 \mathrm{~nm}$ were prepared. hMSCs cultured on 50 and $62 \mathrm{~nm}$ surfaces showed smaller spread areas when compared to cells cultured on 34 or 44 nm surfaces. Moreover cells were well spread on $34 \mathrm{~nm}$ surfaces compared to $62 \mathrm{~nm}$ surfaces. Also immunostaining for focal adhesion kinase (FAK) showed a decreased expression in hMSCs with interligand distance greater than $50 \mathrm{~nm}$. Further morphological changes were induced by ligand spacing leading to differences in migration and differentiation behavior. An increase in migration rate was observed on surfaces with ligand spacings between 34 and $50 \mathrm{~nm}$ with a subsequent decrease on surfaces with a $62 \mathrm{~nm}$ interligand spacings. An increase in migration on 34 and $50 \mathrm{~nm}$ surfaces could be due to an elevated adhesion strength providing optimum strength to support cells but with efficient turnover of focal adhesions. Further increase in ligand spacing was proposed to show less stable protrusions and therefore a slower migration could be observed. More interestingly osteogenic differentiation was found to be pronounced on surfaces where RGD was spaced by $34 \mathrm{~nm}$ while in the case of $62 \mathrm{~nm}$ spaced RGD ligands a more adipogenic differentiation was observed. Furthermore, when cells were incubated on surfaces with a double osteo- and adipogenic induction medium, cells showed osteogenic differentiation on the $34 \mathrm{~nm}$ spacing and adipogenic differentation on the $62 \mathrm{~nm}$ spacing. These findings prove that the lateral spacing of ligands influences stem cell differentiation.

\subsubsection{Ligand density variations on peptide amphiphiles}

Peptide amphiphiles combine the features of amphiphilic surfactants with the biological functions of specific peptides and can therefore self-assemble into biologically active nanostructures (Figure 2.1c). ${ }^{59}$ A specific biological function of a monovalent peptide can easily be amplified in the self-assembled nanostructures by mixing in a high amount of peptide amphiphiles containing the binding epitope to create a high local surface density of the peptide ligands at the exterior of the nanostructure. Depending on the epitope density and dynamics the cell response to the PA nanostructures can be regulated. ${ }^{60}$ When PAs containing RGD ligands were presented to cells, cell adhesion 
was found to be integrin dependent and modulated by changing the ligand density. The ligand density on the PA nanostructures was tuned in this case by changing the peptide architecture from a linear peptide exposing only one RGD per epitope (162 pmol/ $\mathrm{cm}^{2}$ ) to a branched peptide having two RGD per epitope $\left(325 \mathrm{pmol} / \mathrm{cm}^{2}\right) .{ }^{61,62}$ In another example the TGF- $\beta 1$ activity could be controlled by adjusting the TGF- $\beta$ binding epitope density on the PA nanostructures. ${ }^{63}$ When the TGF- $\beta$ binding epitopes were presented on the PA nanostructures in $10 \mathrm{~mol} \%$ ligand density, TGF- $\beta$ was bound and released slower when compared to a control epitope. These TGF- $\beta$ binding PA nanostructures could also bind exogenous TGF- $\beta$ and this possibility was under in vivo conditions leading to a significant regenerative response without addition of exogenous TGF- $\beta .^{63}$ In some cases changes in length, shape, charge and ligand density of active ligand can lead to differences in mechanical properties of PAs. ${ }^{45,46}$ These changes in material stiffness can profoundly influence the cell signaling thus interfering with the effect of ligand density. This problem can be rectified by mixing the peptide with nonbioactive peptide such that the gelation property of the scaffold is mainly controlled by a non-bioactive peptide. ${ }^{64}$ Due to the tremendous modularity potential of peptide amphiphiles, ligand density variation technology using PAs offers a straightforward strategy to further optimize treatments in which injectable scaffolds can lead to positive clinical outcomes e.g. in the treatment of osteoarthritis or ophthalmological disorders. For more detailed information on PAs and their behavior with cells the reader is referred to a recent review. ${ }^{59}$

\subsubsection{Ligand density variations in peptide nucleic acids}

Peptide nucleic acid (PNA) molecules are similar to DNA molecules, but have a chargeneutral backbone consisting of peptide-like molecules and side chains bearing nucleobases. These structural features of PNA molecules allow them to bind more stably with complementary DNA. With some minor modifications in the structure of PNA, active ligands can be attached to the side chain. In a report from Appella and coworkers side chains of PNA molecule were modified with l-lysine derivative were used for displaying ligands (Figure 2.9). ${ }^{65}$ It was observed that this modification of the PNA side chain did not interfere with the binding of PNA with complementary DNA. The l-lysine handle was further conjugated to an integrin antagonist ligand with known 
anticancer activity. ${ }^{[66]}$ Using this approach, four different PNA molecules with a different number of ligands attached to its side chain were synthesized. These modified PNAs were then complexed with linear DNA strands of different lengths (Figure 2.9b,c). With these DNA-PNA nanoscaffolds the ligand density of the integrin antagonist was varied from 1 to 45 with high spatial precision. These nanoscaffolds were then screened for their activity $\left(I C_{50}\right)$ using a cell-based assay. Upon increasing the number of ligands per PNA molecule, an increase in its activity was observed. A similar increase in activity was observed when the PNA repeats (number of PNAs attached) on DNA strands were increased. From these observations a clear influence of ligand density per nanoscaffold on its activity was observed. Moreover, scaffolds with maximum activity were screened in vivo. An elevated biological activity of the nanoscaffolds with conjugated ligands was observed when compared to unconjugated monovalent ligands.

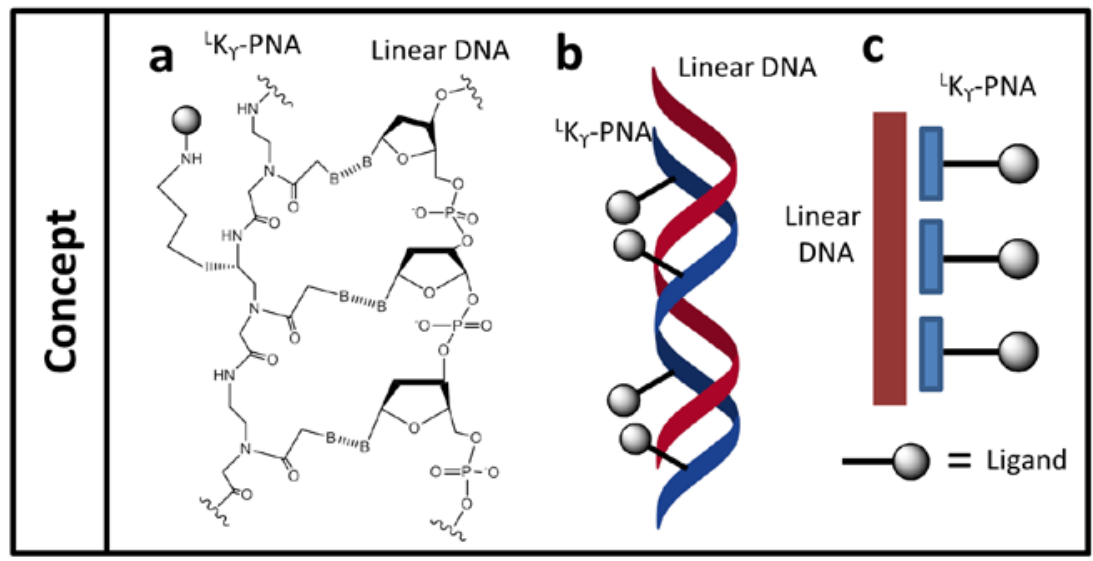

Figure 2.9 - a) Controlling the display of ligand density on PNA nanoscaffolds. Chemical structure of ${ }^{\mathrm{L}} \mathrm{K}_{\Upsilon}$-PNA bound to DNA. b, c) Ribbon and cartoon diagrams of four ${ }^{\mathrm{L}} \mathrm{K}_{\gamma}$-PNAs (each bearing one ligand) bound to a linear DNA.

In an alternative strategy from the same group, peptide nucleic acids (PNA) were conjugated to DNA to control the density of ligands and to study their effect on G protein coupled receptor (GPCR) induced signaling pathways. ${ }^{67}$ This type of receptors were present on mammalian cell surfaces and is responsible for collecting information from outside the cell to regulate the cell's internal machinery. In order to probe the 
influence of ligand spacing on a GPCR pathway, programmable multivalent scaffolds were developed as discussed above. ${ }^{65,68}$

The number of ligands on these scaffolds was varied in this case from 1 to 15 yielding different degrees of interligand spacings. Initial binding studies showed that the PNA:DNA complex bearing two ligands bound significantly better to GPCR than a corresponding monovalent complex. Next, the influence of the interligand spacing on the binding affinity was studied. To this end, a series of bivalent constructs was synthesized where the position of the ligand on PNA was systematically changed to generate complexes with different interligand spacings (Figure 2.10). Surprisingly, DNA was found to have a detrimental effect on the binding of receptors at low valencies. Replacing DNA by complementary PNA yielded L-PNA:PNA complexes that were identified as the weakest binders in the case of the highest and lowest interligand spacing while strong binders were found for the intermediate spacings. Owing to excellent reliability of programming ligand density in spatially designed nucleic acid scaffolds, a model system is at hand to interrogate the effect of variation in ligand density for other ligand receptor systems. Moreover these multivalent LPNA:PNA complexes can be used to design the next generation of biosensors by incorporating them into electrochemical, optoelectronic and microarray-based sensing devices while ligand density variation can be used to maximize the signal to noise ratio of the final biosensing device. ${ }^{69}$ 


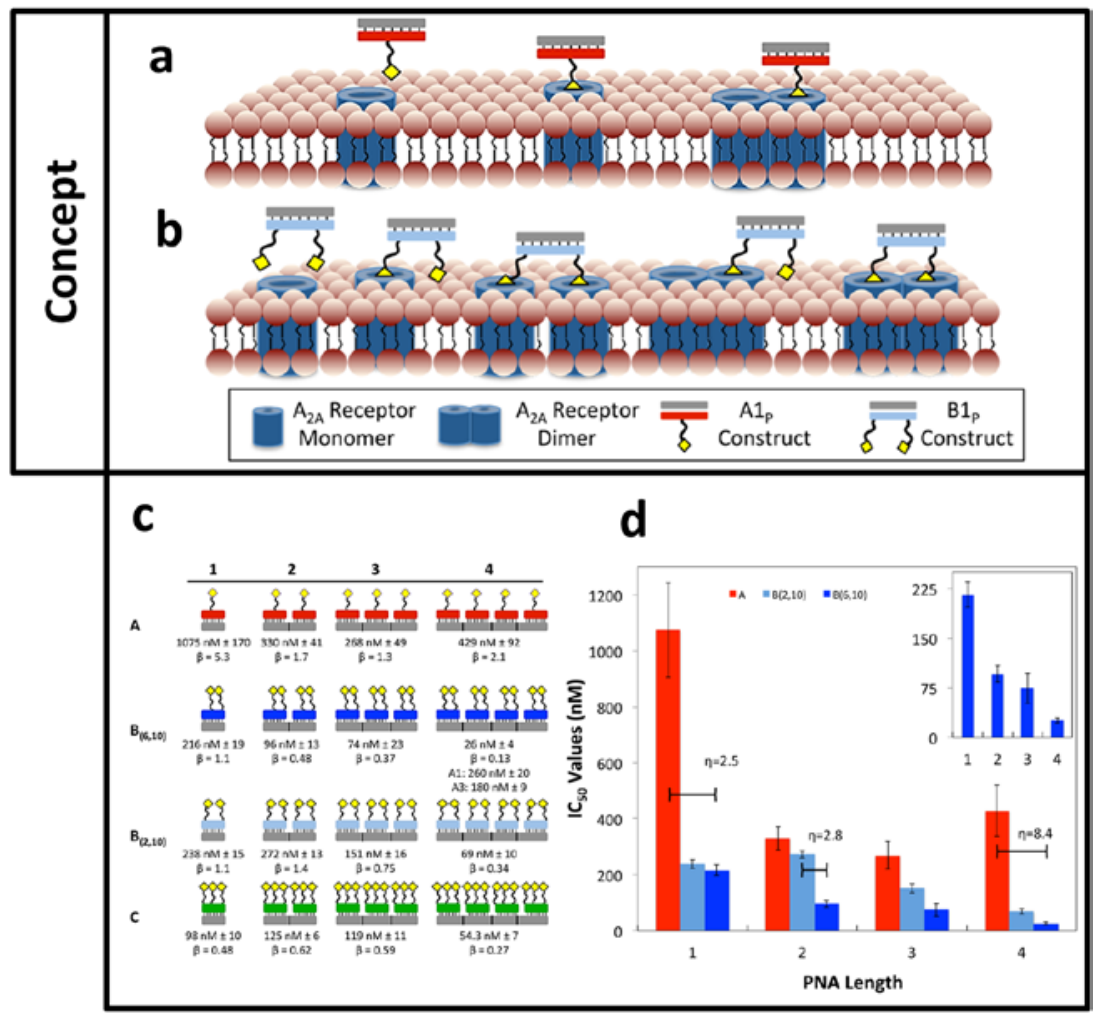

Figure 2.10 - A statistical model showing different binding states between L-PNA:PNA and the receptor. A subset of these states is highlighted for the a) monovalent complex and b) bivalent complexes. L-PNA:DNA multivalent library and landscape. c) L-PNA:PNA multivalent library with the associated $I C_{50}$ and $\beta$ values. Complex $\mathrm{B}(6,10) 4 \mathrm{P}$ was also screened for binding to other human AR subtypes A1AR (260 nM) and A3AR (180 nM). d) Multivalent landscape highlighting the relationships between the A (red), B(2,10) (light blue), B(6,10) (dark blue), and C (green) type L-PNA constructs when annealed to various lengths of complementary PNA. The inset shows the progressively increasing binding affinity of the B $(6,10)$ family as the length of the PNA complement is increased. Key $\eta$ values signal an increase in the individual ligand binding affinity. (Reprinted with permission from J. Am. Chem. Soc., 2014, 136, 12296-12303. Copyrights 2014 ACS.) 


\subsection{Surfaces with uniform arrangement of ligands}

\subsubsection{Ligand density variations on nanocorals}

Nanocorals are 3D nanostructured platforms consisting of uniform arrays of gold dots on solid surfaces. They are generally fabricated using block copolymer micellar nanolithography. ${ }^{70}$ Using this technique, very uniform arrangements of gold dots on solid surfaces can be obtained. Nanopattern formation involves the formation of 2D close-packed layers of block co-polymer micelles with gold particles followed by hydrogen plasma etching and passivation. The ligands of interest are then grafted on to the gold dots using thiol-gold chemistry. Dunlop and coworkers ${ }^{71}$ have investigated the influence of ligand spacing on the cellular response when T cell and natural killer (NK) cell were incubated on these platforms. To study T-cell stimulation gold particles were functionalized with $\mathrm{F}\left(\mathrm{ab}^{\prime}\right)_{2}$ fragments derived from the UCHT-1 antibody that binds the CD3 $\varepsilon$ component of the TCR complex. ${ }^{72} \mathrm{NK}$ cell stimulating surfaces were prepared by functionalizing gold nanoparticles with a biotin alkanethiol, followed by streptavidin and biotinylated CD16-binding antibodies (3G8 mAb and rituximab). When these surfaces were plated with either T cells or NK cells, both cell types showed a decrease in signaling level upon increase in ligand spacing (or decrease in ligand density). Spacing between two gold nanoclusters of around $69 \mathrm{~nm}$ was sufficient to decrease $\mathrm{T}$ cell signaling down to background levels, while in the case of NK cells the spacing was found to be around $104 \mathrm{~nm}$. These results show strong dependence of immunoreceptor signaling on ligand spacing (or density).

\subsubsection{Spatial clustering of ligands}

The examples discussed above and other literature data show the extraordinary capacity of cells to respond differentially to differences in average ligand densities on surfaces. However, cells are known to be responsive to controlled spatial groupings of ligands (Figure 2.1). ${ }^{9,10}$ To achieve spatial grouping of ligands, Spatz and coworkers have adopted the self-assembly of gold-loaded block copolymer micelles to achieve lateral distances of 20-250 $\mathrm{nm}$ between Au clusters depending on the molecular weight of the polymers (figure 2.11). ${ }^{73}$ 


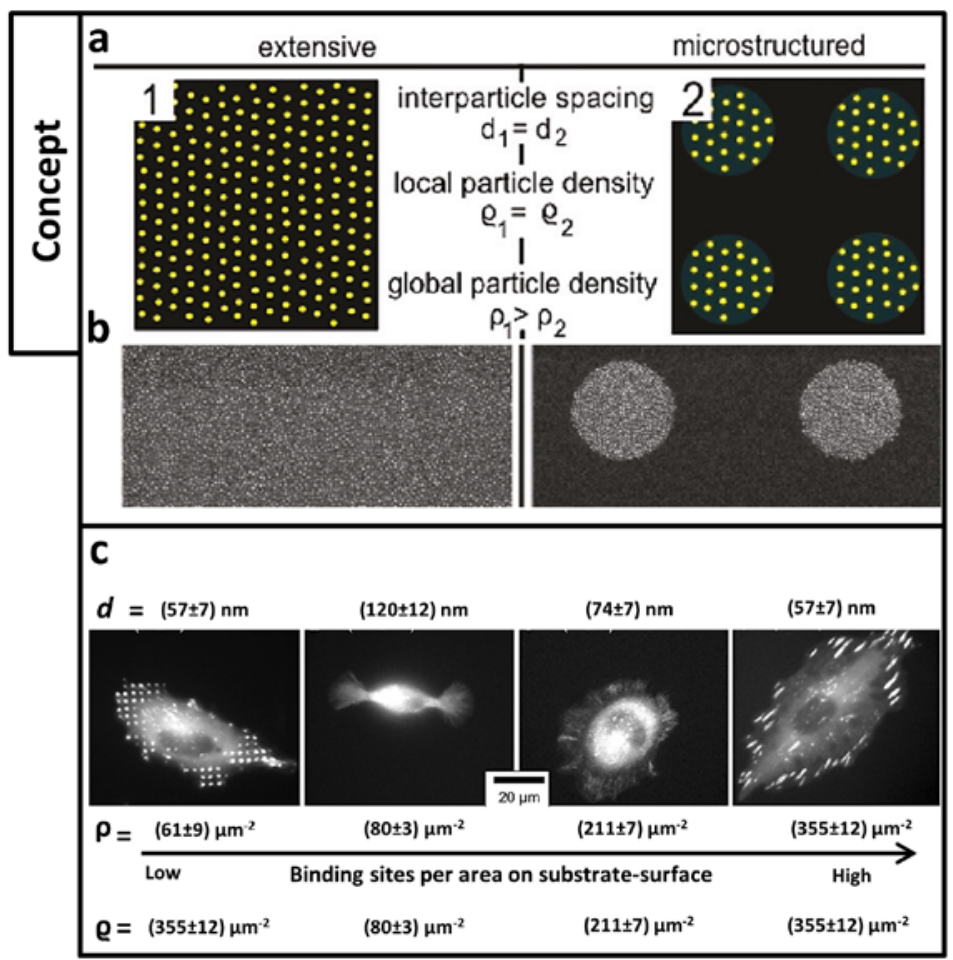

Figure 2.11 - a) Scheme showing hierarchical organization of nanoparticle arrays in microdomains leading to the separation of local particle density (interparticle distance) from global particle density. b) Actual images of extensive and micronanostructured surfaces. c) Micrographs of REF-YFP-Pax cells $12 \mathrm{~h}$ after seeding on micro/nanostructured surfaces or extensive nanopattern surfaces with different interligand spacings (denoted by $d$ ). Focal adhesions (visible as bright patches on the cell periphery) develop well on substrates with an interligand spacing of less than $70 \mathrm{~nm}$. Underneath the global particle density (denoted by $\rho$ ) and local particle density (denoted by Q) for each surface is given. (Reprinted with permission from Nano Lett., 2011, 11, 1469-1476. Copyrights 2011 ACS.).

The global density of the particles was reduced during a photolithography step by partially removing them. This process created islands of nanoparticle arrays of ca. 1.5 $\mu \mathrm{m}$ separated by empty regions of about $1.7 \mu \mathrm{m}$ (Figure 2.11a). Arrays were made that differ in inter particle spacing $d$ and in ligand spacing $\rho$ (global) and $\varrho$ (local). After connecting RGD ligands to the gold particles, rat embryonic fibroblasts were grown 
over the arrays. A remarkable dependence of ligand spacing on cellular adhesion was observed. On all surfaces the projected cell area increased as a function of time.

After 6 h cell spreading was similar on the surfaces with low global ligand densities. While after longer incubation times (12 h or $24 \mathrm{~h}$ ) cells started discriminating between minute changes in ligand spacing. The spreading area of cells on surfaces with a smaller interligand spacing $(57 \mathrm{~nm})$ was significantly larger than on surfaces with a larger interligand spacing (70 nm). In case of surfaces with an interligand spacing of less than $60 \mathrm{~nm}$, it was observed that cells spread more on an extensive uniformly patterned surface compared to a micro/nanostructured surface. On surfaces with an interligand spacing greater than $70 \mathrm{~nm}$ focal adhesion formation was found to be suppressed compared to surfaces where ligands are spaced $57 \mathrm{~nm}$ apart. The shape of focal adhesion formation was found to be dictated by the geometry of the surface, i.e. "island"-like or uniform. However, microstructuring of the surface did not influence the number of focal adhesions formed: in the case of micro/nanostructured surfaces, the strength of focal adhesions was similar to uniformly covered surfaces. These results indicate that the finding on patterned surfaces, where an increase in global ligand density corresponds to an increase in adhesion strength until a saturation level, does not apply to micro/nanostructured surfaces. This variation in local vs global ligand density provides a novel aspect in the concept and understanding of the effects of variation in ligand spacing on cell adhesion strength and focal adhesion formation. This nanoscale view at focal adhesion formation as a function of ligand spacing can help to develop novel materials for biomedical applications as well as to design implantable devices. For example, nanodot organization and ligand density variation technology can be implemented using standard fabrication techniques in designing surfaces of cardiovascular implants with an optimal number of effective integrin clusters necessary to achieve endothelial cell adhesion and promote endothelization of the implant.

\subsection{Conclusions and future perspectives}

The literature examples discussed above have shown the extraordinary capacity of cells to respond to ligand density changes at various length scales. Ligand density variation which acted as an extracellular signal not only influenced characteristics of cells like morphology and migration but also influenced cell responses such as differentiation, 
proliferation and apoptosis. Biochemical cues provided to cells in terms of differences in ligand density also showed to influence recruitment and activity of biomolecules like growth factors and proteoglycans. These differences in levels of sensitization of ligands was shown to influence the extent of cellular signaling. Some studies investigating the impact of ligand density on cell adhesion showed that cells were able to spatially recognize the changes in density of ligands. Interestingly a window of high and low density of ligands is present, i.e. above and below these threshold limits of ligand spacing no cell adhesion was observed. Moreover structural changes in the nature of the interface, i.e. the dimensionality (2D or 3D) or topography have been shown to impact the influence of ligand density. Different responses have been observed in the case of interfaces where ligand density is varied along with these parameters.

So far many bioactive interfaces have been reported that are limited in their ability to immobilize the ligands of interest in varying density. With the emerging classes of interfaces discussed in this review, it is possible to precisely vary the density or spacing of ligands in both 2D and 3D fashion. The translation of ligand density variation from $2 \mathrm{D}$ to $3 \mathrm{D}$ surfaces is, however, challenging due to the presence of uneven and curved edges on surfaces of most if not all medical implants and devices. Moreover, extrapolating outcomes of studies of ligand density variation technology on flat surfaces, will need verification on final 3D device structures. Likewise, substantial work will be required to investigate the impact of ligand density variation on medical devices and implants under in vivo conditions, and to study in greater detail the effect of subtle changes in ligand density on biological developmental processes like organogenesis. All of these studies will bring the design of biomaterials to a higher level that is more physiologically informed about optimal ligand densities to maximize the bio-efficacy upon implantation.

\subsection{References}

1. Changeux, J.-P., Thiéry, J., Tung, Y. \& Kittel, C. On the cooperativity of biological membranes. Proceedings of the National Academy of Sciences 57, 335-341 (1967).

2. Groves, J.T. Molecular organization and signal transduction at intermembrane junctions. Angewandte Chemie 117, 3590-3605 (2005). 
3. Heldin, C.-H. Dimerization of cell surface receptors in signal transduction. Cell 80, 213-223 (1995).

4. Weiss, A. \& Schlessinger, J. switching signals on or off by receptor dimerization. Cell 94, 277-280 (1998).

5. Fasting, C. Schalley, C. A. \& Haag, r. Multivalency as a chemical organization and action principle. Angewandte Chemie International Edition 51, 1047210498 (2012).

6. Geiger, B., Bershadsky, A., Pankov, R. \& Yamada, K.M. Transmembrane crosstalk between the extracellular matrix and the cytoskeleton. Nat Rev Mol Cell Biol 2, 793-805 (2001).

7. Engler, A.J., Sen, S., Sweeney, H.L. \& Discher, D.E. Matrix elasticity directs stem cell lineage specification. Cell 126, 677-689 (2006).

8. Zaidel-Bar, R., Itzkovitz, S., Ma'ayan, A., Iyengar, R. \& Geiger, B. Functional atlas of the integrin adhesome. Nat Cell Biol 9, 858-867 (2007).

9. Geiger, B., Spatz, J.P. \& Bershadsky, A.D. Environmental sensing through focal adhesions. Nat Rev Mol Cell Biol 10, 21-33 (2009).

10. Geiger, B., Yehuda-Levenberg, S. \& Bershadsky, A.D. Molecular interactions in the submembrane plaque of cell-cell and cell-matrix adhesions. Cells Tissues Organs 154, 46-62 (1995).

11. Cavalcanti-Adam, E.A. et al. Cell spreading and focal adhesion dynamics are regulated by spacing of integrin ligands. Biophysical Journal 92, 2964-2974 (2007).

12. Koepsel, J.T. \& Murphy, W.L. A chemically-defined screening platform reveals behavioral similarities between primary human mesenchymal stem cells and endothelial cells. Integrative Biology 4, 1508-1521 (2012).

13. Shabbir, S.H., Eisenberg, J.L. \& Mrksich, M. An Inhibitor of a cell adhesion receptor stimulates cell migration. Angewandte Chemie International Edition 49, 7706-7709 (2010).

14. Kiessling, L.L. \& Grim, J.C. Glycopolymer probes of signal transduction. Chemical Society Reviews 42, 4476-4491 (2013).

15. Slaughter, B.V., Khurshid, S.S., Fisher, O.Z., Khademhosseini, A. \& Peppas, N.A. Hydrogels in Regenerative Medicine. Advanced Materials 21, 3307-3329 (2009). 
16. Mitragotri, S. \& Lahann, J. Physical approaches to biomaterial design. Nat Mater 8, 15-23 (2009).

17. Houseman, B.T., Gawalt, E.S. \& Mrksich, M. Maleimide-functionalized selfassembledmonolayers for the preparation of peptide and carbohydrate biochips. Langmuir 19, 1522-1531 (2002).

18. Geiger, B., Tokuyasu, K.T., Dutton, A.H. \& Singer, S.J. Vinculin, an intracellular protein localized at specialized sites where microfilament bundles terminate at cell membranes. Proceedings of the National Academy of Sciences 77, 4127-4131 (1980).

19. Hudalla, G.A. \& Murphy, W.L. Immobilization of peptides with distinct biological activities onto stem cell culture substrates using orthogonal chemistries. Langmuir 26, 6449-6456 (2010).

20. Hudalla, G.A. \& Murphy, W.L. Using “click” chemistry to prepare sam substrates to study stem cell adhesion. Langmuir 25, 5737-5746 (2009).

21. Koepsel, J.T. \& Murphy, W.L. Patterning discrete stem cell culture environments via localized self-assembled monolayer replacement. Langmuir 25, 12825-12834 (2009).

22. Kilian, K.A. \& Mrksich, M. Directing stem cell fate by controlling the affinity and density of ligand-receptor interactions at the biomaterials interface. Angewandte Chemie International Edition 51, 4891-4895 (2012).

23. Mas-Moruno, C., Rechenmacher, F. \& Kessler, H. Cilengitide: The first antiangiogenic small molecule drug candidate. design, synthesis and clinical evaluation. Anti-Cancer Agents in Medicinal Chemistry 10, 753-768 (2010).

24. Huebsch, N. et al. Harnessing traction-mediated manipulation of the cell/matrix interface to control stem-cell fate. Nat Mater 9, 518-526 (2010).

25. Su, J.-L. et al. CYR61 regulates bmp-2-dependent osteoblast differentiation through the $\alpha \mathrm{v} \beta 3$ integrin/integrin-linked kinase/erk pathway. Journal of Biological Chemistry 285, 31325-31336 (2010).

26. Teng, Y.D. Functional recovery following traumatic spinal cord injury mediated by a unique polymer scaffold seeded with neural stem cells. Proceedings of the National Academy of Sciences 99, 3024-3029 (2002). 
27. Nowinski, A.K., Sun, F., White, A.D., Keefe, A.J. \& Jiang, S. Sequence, structure, and function of peptide self-assembled monolayers. Journal of the American Chemical Society 134, 6000-6005 (2012).

28. Le Saux, G., Magenau, A., Böcking, T., Gaus, K. \& Gooding, J.J. The relative importance of topography and RGD ligand density for endothelial cell adhesion. PLoS ONE 6, e21869 (2011).

29. Curtis, A.S.G. \& Wilkinson, C.D.W. Reactions of cells to topography. Journal of Biomaterials Science, Polymer Edition 9, 1313-1329 (1998).

30. Martinez-Veracoechea, F.J. \& Frenkel, D. Designing super selectivity in multivalent nano-particle binding. Proceedings of the National Academy of Sciences 108, 10963-10968 (2011).

31. Orgovan, N. et al. Dependence of cancer cell adhesion kinetics on integrin ligand surface density measured by a high-throughput label-free resonant waveguide grating biosensor. Sci. Rep. 4 (2014).

32. Reinhart-King, C.A., Dembo, M. \& Hammer, D.A. The dynamics and mechanics of endothelial cell spreading. Biophysical Journal 89, 676-689 (2005).

33. Dubin-Thaler, B.J., Giannone, G., Döbereiner, H.-G. \& Sheetz, M.P. Nanometer analysis of cell spreading on matrix-coated surfaces reveals two distinct cell states and steps. Biophysical Journal 86, 1794-1806 (2004).

34. Frisch, T. \& Thoumine, O. Predicting the kinetics of cell spreading. Journal of Biomechanics 35, 1137-1141 (2002).

35. Tayalia, P. \& Mooney, D.J. Controlled growth factor delivery for tissue engineering. Advanced Materials 21, 3269-3285 (2009).

36. Li, L., Klim, J.R., Derda, R., Courtney, A.H. \& Kiessling, L.L. Spatial control of cell fate using synthetic surfaces to potentiate TGF- $\beta$ signaling. Proceedings of the National Academy of Sciences (2011).

37. Li, L., Orner, B.P., Huang, T., Hinck, A.P. \& Kiessling, L.L. Peptide ligands that use a novel binding site to target both TGF-[ $\beta]$ receptors. Molecular BioSystems 6, 2392-2402 (2010).

38. Hudalla, G.A., Koepsel, J.T. \& Murphy, W.L. Surfaces that sequester serumborne heparin amplify growth factor activity. Advanced Materials 23, 54155418 (2011). 
39. Le Saux, G. Spacing of integrin ligands influences signal transduction in endothelial cells. Biophysical Journal 101, $764-773$ (2011).

40. Melkoumian, Z. et al. Synthetic peptide-acrylate surfaces for long-term selfrenewal and cardiomyocyte differentiation of human embryonic stem cells. Nat Biotech 28, 606-610 (2010).

41. Stratman, A.N. et al. Endothelial cell lumen and vascular guidance tunnel formation requires MT1-MMP-dependent proteolysis in 3-dimensional collagen matrices, Vol. 114. (2009).

42. Sacharidou, A. et al. Endothelial lumen signaling complexes control 3D matrix-specific tubulogenesis through interdependent Cdc42- and MT1-MMPmediated events, Vol. 115. (2010).

43. Hanjaya-Putra, D. et al. Vascular endothelial growth factor and substrate mechanics regulate in vitro tubulogenesis of endothelial progenitor cells. Journal of Cellular and Molecular Medicine 14, 2436-2447 (2010).

44. Koepsel, J.T., Nguyen, E.H. \& Murphy, W.L. Differential effects of a soluble or immobilized VEGFR-binding peptide. Integrative Biology 4, 914-924 (2012).

45. Shamloo, A. \& Heilshorn, S.C. Matrix density mediates polarization and lumen formation of endothelial sprouts in VEGF gradients. Lab on a Chip 10, 3061-3068 (2010).

46. Calvani, M., Rapisarda, A., Uranchimeg, B., Shoemaker, R.H. \& Melillo, G. Hypoxic induction of an HIF-1 $\alpha$-dependent bFGF autocrine loop drives angiogenesis in human endothelial cells, Vol. 107. (2006).

47. Abaci, H.E., Truitt, R., Tan, S. \& Gerecht, S. Unforeseen decreases in dissolved oxygen levels affect tube formation kinetics in collagen gels, Vol. 301. (2011).

48. Nguyen, E.H., Zanotelli, M.R., Schwartz, M.P. \& Murphy, W.L. Differential effects of cell adhesion, modulus and VEGFR-2 inhibition on capillary network formation in synthetic hydrogel arrays. Biomaterials 35, 2149-2161 (2014).

49. Musah, S. et al. Glycosaminoglycan-binding hydrogels enable mechanical control of human pluripotent stem cell self-renewal. ACS Nano 6, 10168-10177 (2012). 
50. Murphy, W.L., McDevitt, T.C. \& Engler, A.J. Materials as stem cell regulators. Nat Mater 13, 547-557 (2014).

51. Wen, J.H. et al. Interplay of matrix stiffness and protein tethering in stem cell differentiation. Nat Mater 13, 979-987 (2014).

52. Rowlands, A.S., George, P.A. \& Cooper-White, J.J. Directing osteogenic and myogenic differentiation of MSCs: interplay of stiffness and adhesive ligand presentation, Vol. 295. (2008).

53. Winer, J.P., Janmey, P.A., McCormick, M.E. \& Funaki, M. Bone marrowderived human mesenchymal stem cells become quiescent on soft substrates but remain responsive to chemical or mechanical stimuli. Tissue Engineering Part A 15, 147-154 (2008).

54. Cameron, A.R., Frith, J.E. \& Cooper-White, J.J. The influence of substrate creep on mesenchymal stem cell behaviour and phenotype. Biomaterials 32, 5979-5993 (2011).

55. Cukierman, E., Pankov, R., Stevens, D.R. \& Yamada, K.M. Taking cell-matrix adhesions to the third dimension. Science 294, 1708-1712 (2001).

56. Kantawong, F. et al. Whole proteome analysis of osteoprogenitor differentiation induced by disordered nanotopography and mediated by ERK signalling. Biomaterials 30, 4723-4731 (2009).

57. Yim, E.K.F., Darling, E.M., Kulangara, K., Guilak, F. \& Leong, K.W. Nanotopography-induced changes in focal adhesions, cytoskeletal organization, and mechanical properties of human mesenchymal stem cells. Biomaterials 31, 1299-1306 (2010).

58. Frith, J.E., Mills, R.J. \& Cooper-White, J.J. Lateral spacing of adhesion peptides influences human mesenchymal stem cell behaviour. Journal of Cell Science 125, 317-327 (2012).

59. Cui, H., Webber, M.J. \& Stupp, S.I. Self-assembly of peptide amphiphiles: From molecules to nanostructures to biomaterials. Peptide Science 94, 1-18 (2010).

60. Storrie, H. et al. Supramolecular crafting of cell adhesion. Biomaterials 28, 4608-4618 (2007). 
61. Guler, M.O., Soukasene, S., Hulvat, J.F. \& Stupp, S.I. Presentation and recognition of biotin on nanofibers formed by branched peptide amphiphiles. Nano Letters 5, 249-252 (2004).

62. Guler, M.O. et al. Presentation of RGDS epitopes on self-assembled nanofibers of branched peptide amphiphiles. Biomacromolecules 7, 1855-1863 (2006).

63. Shah, R.N. et al. Supramolecular design of self-assembling nanofibers for cartilage regeneration. Proceedings of the National Academy of Sciences 107, 3293-3298 (2010).

64. Anderson, J.M., Andukuri, A., Lim, D.J. \& Jun, H.-W. Modulating the gelation properties of self-assembling peptide amphiphiles. ACS Nano 3, 3447-3454 (2009).

65. Englund, E.A. et al. Programmable multivalent display of receptor ligands using peptide nucleic acid nanoscaffolds. Nat Commun 3, 614 (2012).

66. Mas-Moruno, C., Rechenmacher, F. \& Kessler, H. Cilengitide: The first antiangiogenic small molecule drug candidate. design, synthesis and clinical evaluation. Anti-Cancer Agents in Medicinal Chemistry 10, 753-768 (2010).

67. Dix, A.V. et al. Programmable nanoscaffolds that control ligand display to a gprotein-coupled receptor in membranes to allow dissection of multivalent effects. Journal of the American Chemical Society 136, 12296-12303 (2014).

68. Englund, E.A. \& Appella, D.H. $\gamma$-Substituted peptide nucleic acids constructed from l-lysine are a versatile scaffold for multifunctional display. Angewandte Chemie International Edition 46, 1414-1418 (2007).

69. Briones, C. \& Moreno, M. Applications of peptide nucleic acids (PNAs) and locked nucleic acids (LNAs) in biosensor development. Anal Bioanal Chem 402, 3071-3089 (2012).

70. Lohmüller, $\mathrm{T}$. et al. Nanopatterning by block copolymer micelle nanolithography and bioinspired applications. Biointerphases 6, MR1-MR12 (2011).

71. Delcassian, D. et al. Nanoscale ligand spacing influences receptor triggering in t cells and nk cells. Nano Letters 13, 5608-5614 (2013).

72. Arnett, K.L., Harrison, S.C. \& Wiley, D.C. Crystal structure of a human CD3$\varepsilon / \delta$ dimer in complex with a UCHT1 single-chain antibody fragment. 
Proceedings of the National Academy of Sciences of the United States of America 101, 16268-16273 (2004).

73. Deeg, J.A. et al. Impact of local versus global ligand density on cellular adhesion. Nano Letters 11, 1469-1476 (2011). 


\title{
Chapter 3
}

\section{Employing the weakly binding aromatic amino acids for multivalent peptide complexation with $\beta$ - \\ CD printboards}

\begin{abstract}
In this chapter we show the development of the concept of programmable multivalency to modulate the binding affinity of multivalent peptide guests with a receptor-modified surface. Multivalent peptides with 3-6 aromatic amino acid (tyrosine, Y) units were synthesized. Aromatic amino acids are known to form host-guest complexes with $\beta$ cyclodextrin ( $\beta$-CD). When these multivalent peptides were allowed to interact with a $\beta$ CD self-assembled monolayer (SAM), a linear enhancement of the binding affinity (log $K$ ) was observed upon the addition of each additional Y guest unit in the peptide. When the $\mathrm{Y}$ residues were replaced with other, non-aromatic, amino acids complete loss of binding affinity was observed. a minimum spacing between the $\mathrm{Y}$ units in the peptide was found to be crucial for achieving specific binding of the multivalent peptide with the $\beta$-CD SAM, but longer spacer lengths did not lead to a detectable change in affinity. Moreover, a linear peptide showed a similar affinity as a branched peptide containing the same number of and spacing between the aromatic amino acids.
\end{abstract}




\subsection{Introduction}

The ability to quantitatively tune binding affinities from multiple supramolecular interactions holds high importance in nanofabrication as well as in understanding cellular biology. ${ }^{1}$ Cumulative thermodynamically and kinetically stable interactions from multiple supramolecular interaction pairs have been achieved. ${ }^{2}$ As a step further, with the model of multivalency developed in our group, the overall binding affinity was deciphered into the intrinsic binding constant of each interaction pair, the geometries of the host and guest molecules (which control the effective molarity, $E M$ ), and the number of interaction sites. ${ }^{3}$ With the guidance of this model, a number of delicate nanocomplexes held together by supramolecular interactions have been established. ${ }^{4}$ Even the number of simultaneously bound sites can be deduced from the measured overall binding affinity and the intrinsic affinity provided by each binding site. ${ }^{5,6}$ Conversely, the model can be used to predict affinities of new multivalent guests, thus allowing fine tuning of the overall affinity and dynamics. However, in case of multivalent systems where the individual interaction is weak, such a programmable affinity has not been shown and relationships for different valencies have not been studied so far. At the same time, strong interactions emerging from cumulative multiple weak interactions have attracted ever more attention, since such interactions provide the basis to achieve selectivity in supramolecular systems, ${ }^{7,8}$ and provide biological function. $^{1,7}$

In this chapter, cyclodextrins (CDs) are used as receptors. CDs are cyclic oligo-glucose molecules that exist in three different forms, known as $\alpha-C D, \beta-C D$, and $\gamma-C D$, which have 6,7 , and 8 glucose units, respectively. ${ }^{9} \beta$-CD is particularly appealing as it forms 1:1 inclusion complexes with a large number of guest molecules. ${ }^{7} \beta$-CD molecules can be assembled on the gold surface to form a self-assembled monolayer (SAM) known as the "molecular printboard". ${ }^{10}$ These molecular printboards act as an array of receptor (host) molecules that can be used to study the binding of guest molecules. ${ }^{2}$

Peptides constitute a particularly interesting class of poly-functional guest molecules not only because they can possess a biological function, but also because their structure can easily be programmed to control the position of amino acids in the peptide chain. ${ }^{11}$ However, since the monovalent interactions between amino acids and cyclodextrins are 
too weak to be utilized, the molecular recognition between peptides and cyclodextrins have been sparsely studied. ${ }^{12}$

Here, we demonstrate that by employing the weak molecular recognition between tyrosine (Tyr) and $\beta-C D$, the overall association constant between a peptide containing a different number of tyrosines (multivalent guest) and $\beta$-CD SAMs (multivalent host) can be systematically programmed. We have investigated implications of the spacing between the guest amino acids, the specificity of guest binding (by using serine instead of tyrosine), and the molecular geometry (linear vs branched) on the overall binding affinity with the host surface.

\subsection{Results and discussion}

The peptides employed in this chapter were composed of three kinds of amino acid residues. The phenoxy group of Tyr serves as the guest group expected to bind with $\beta$ CDs; serine (Ser) and glycine (Gly) serve as the spacer and at the same time Ser enhances the solubility of the peptides, while Gly provides flexibility to the peptides because of its small size. Using these amino acids, peptides with the general structural motif Ac(SGGYGGS $)_{n}\left(Y_{n}\right)$ were synthesized. This motif provides a $3.2 \mathrm{~nm}$ separation between adjacent Tyr residues in the peptide. This distance permits the binding of all $\mathrm{Y}$ units in a peptide with $\beta$-CD receptors present in the SAM, in which the center-to-center spacing of adjacent $\beta$-CD cavities is $2.1 \mathrm{~nm}$, as determined previously. ${ }^{13}$ The schematic illustrations of the molecular structures of the peptides and the $\beta$-CD SAM is shown in Figure 3.1. Additionally, the spacing between $\mathrm{Y}$ units was varied, by changing the numbers of S, G, and Y units in the peptides, to provide the structures shown in Table 3.1. 
a

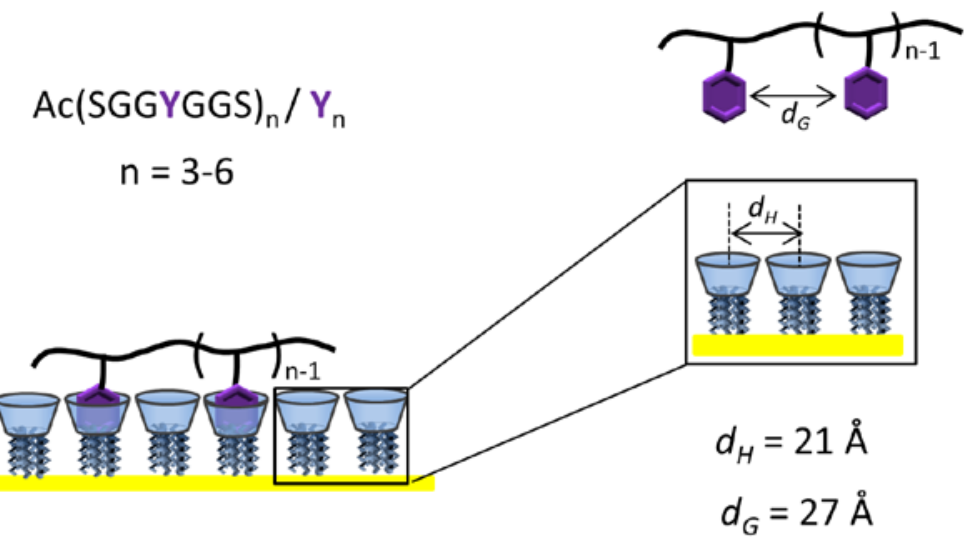

b
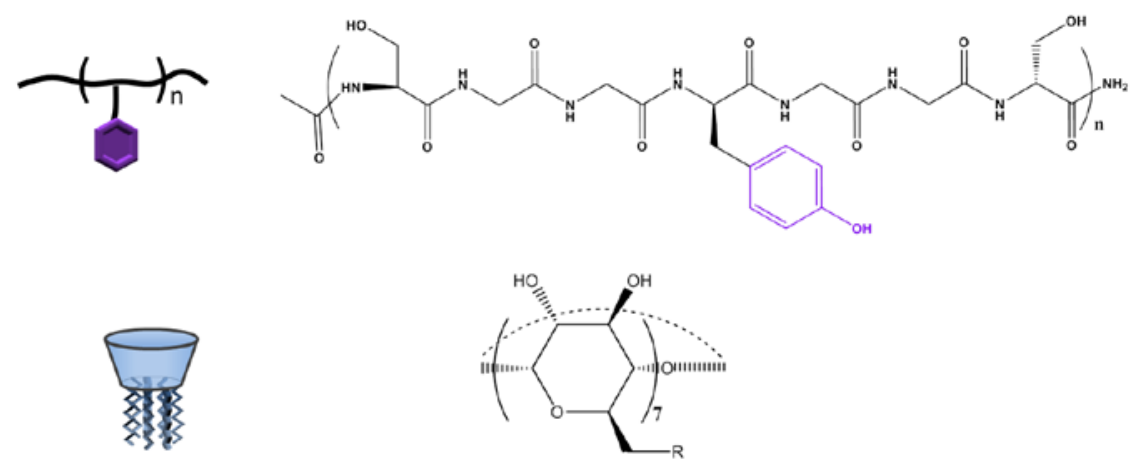

$$
\mathrm{R}=\mathrm{NHC}(\mathrm{O})\left(\mathrm{CH}_{2}\right)_{11} \mathrm{~S}\left(\mathrm{CH}_{2}\right)_{11} \mathrm{CH}_{3}
$$

Figure 3.1 - a) A cartoon representation showing multivalent peptides involved in supramolecular complexation with a $\beta$-CD SAM on gold. b) Chemical structures of guest tyrosine-containing peptides and host $\beta$-CD. 


\begin{tabular}{|c|c|}
\hline Peptide & $d_{G}(\AA)$ \\
\hline $\mathrm{Ac}(\mathrm{SGGYGGS})_{4}$ & 27 \\
\hline $\mathrm{Ac}(\mathrm{SGYGS})_{4}$ & 19 \\
\hline $\mathrm{Ac}(\mathrm{SGY})_{4}$ & 11 \\
\hline $\mathrm{Ac} \mathrm{SYYGYYS}$ & $8(\mathrm{YGY})$ \\
& $4(\mathrm{YY})$ \\
\hline
\end{tabular}

Table 3.1 - Peptides with varying distances between Y units, $d_{G}$, to investigate the effect of intertyrosine spacing on the binding affinity.

\subsubsection{Synthesis of guest peptides and their binding to $\beta$-CD SAMs}

Peptides were synthesized by automated solid phase peptide synthesis using an FmocRink Amide MBHA resin. Purification of the peptides was achieved using reversed phase high performance liquid chromatography (HPLC). The quality of the peptides was checked by analytical HPLC and ESI mass spectrometry. Details are provided in the materials and methods section.

The binding affinities of the synthesized peptides with the $\beta$-CD SAM were determined using surface plasmon resonance (SPR) spectroscopy. Briefly, a solution of a guest molecule is flowed along the surface of bound host ( $\beta$-CD SAM). If the interaction between host and guest takes place, it results in a change of the SPR coupling angle, $\alpha$. Generally, a change of the coupling angle is directly proportional to the surface coverage of the guest. ${ }^{14,15}$ Thus, the angle change, $\Delta \alpha$, was monitored for solutions of increasing guest concentrations until no further angle change was observed, indicating the complete saturation of the host surface with guest molecules as done previously for evaluating other multivalent host-guest interactions at the interface. ${ }^{3}$ As shown in Figure 3.2, solutions with various concentrations of peptides were flowed through the SPR fluidic chamber and the change in coupling angle was recorded. After each concentration and prior to the next, the surfaces were re-generated by rinsing off the peptides using either PBS for the trivalent peptide or buffer with $\beta$-CD for the higher valent peptides. 

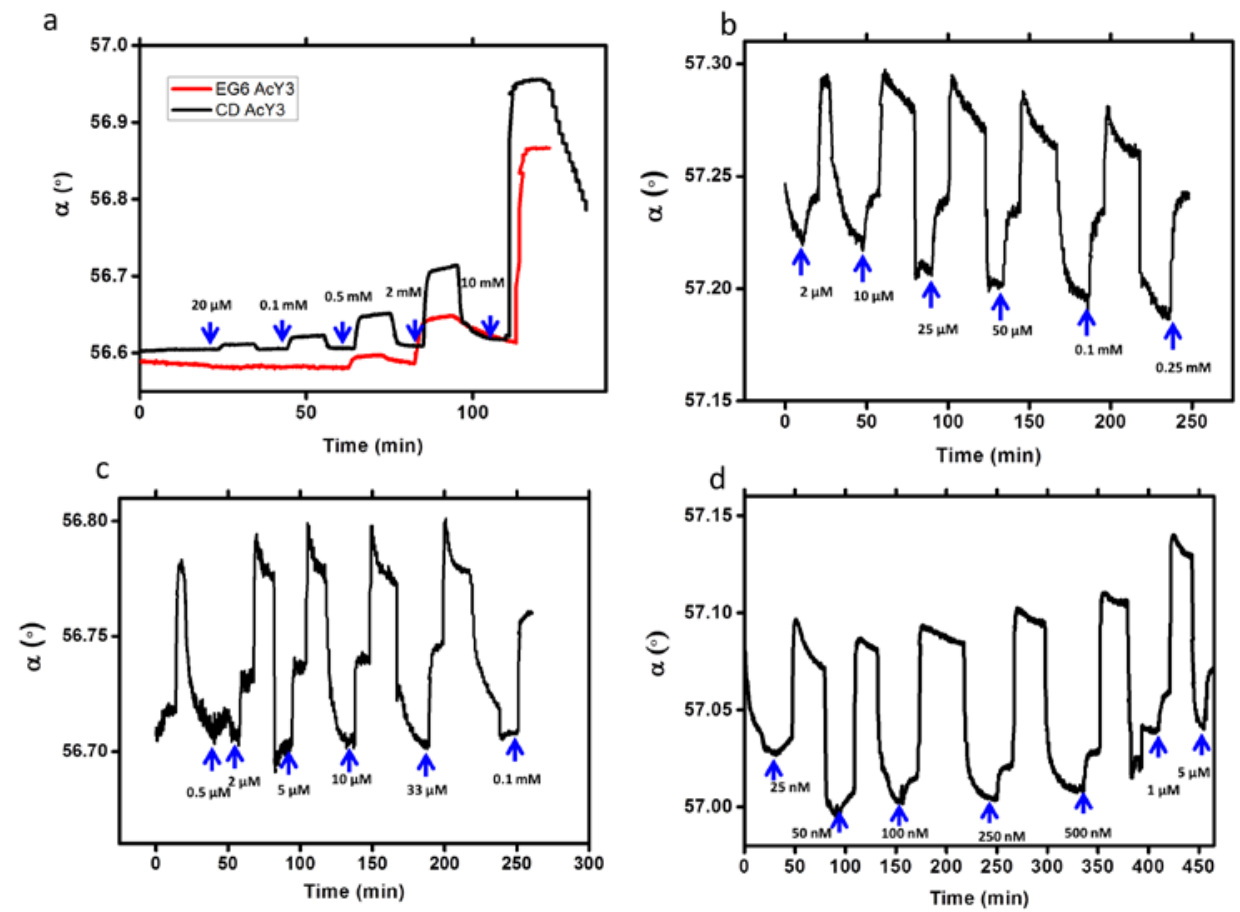

Figure 3.2 - SPR titration data for a) Ac(SGGYGGS) $)_{3}, Y_{3}$; b) Ac(SGGYGGS) $)_{4}, Y_{4}$; c) Ac(SGGYGGS) $)_{5}, Y_{5}$; d) Ac(SGGYGGS) $)_{6}, Y_{6}$, upon binding to a $\beta$-CD SAM (black curves, a-d) or to a $\mathrm{EG}_{6}$ SAM (red curve, a) Graphs show the change in coupling angle as a function of time while different concentrations of peptide, alternated with buffer, are introduced at indicated time points. Following each binding event, the substrate was washed with PBS (a) or $5 \mathrm{mM} \beta$-CD in PBS (b-d) followed by PBS buffer, until a stable baseline was obtained.

Control titration experiments were performed by following the angle change upon flowing solutions with various concentrations of peptide over a reference hexa(ethylene glycol) methyl ether thiol $\left(\mathrm{EG}_{6}\right)$ SAM, where no host-guest interactions can occur. The SPR response on these $\mathrm{EG}_{6}$ surfaces is represented by the red lines in Figure 3.2. The signals observed for the $\mathrm{EG}_{6} \mathrm{SAM}$ at high guest concentrations are attributed to the change of the refractive index of the respective guest solutions, and are used to correct the signals observed for adsorption to the $\beta$-CD SAMs. Changes in the coupling angle $\alpha$ over the $\beta$-CD SAM, after subtraction of the values obtained at the $\mathrm{EG}_{6} \mathrm{SAM}$, were plotted as a function of peptide concentration for all peptide sequences (Figure 3.3). 

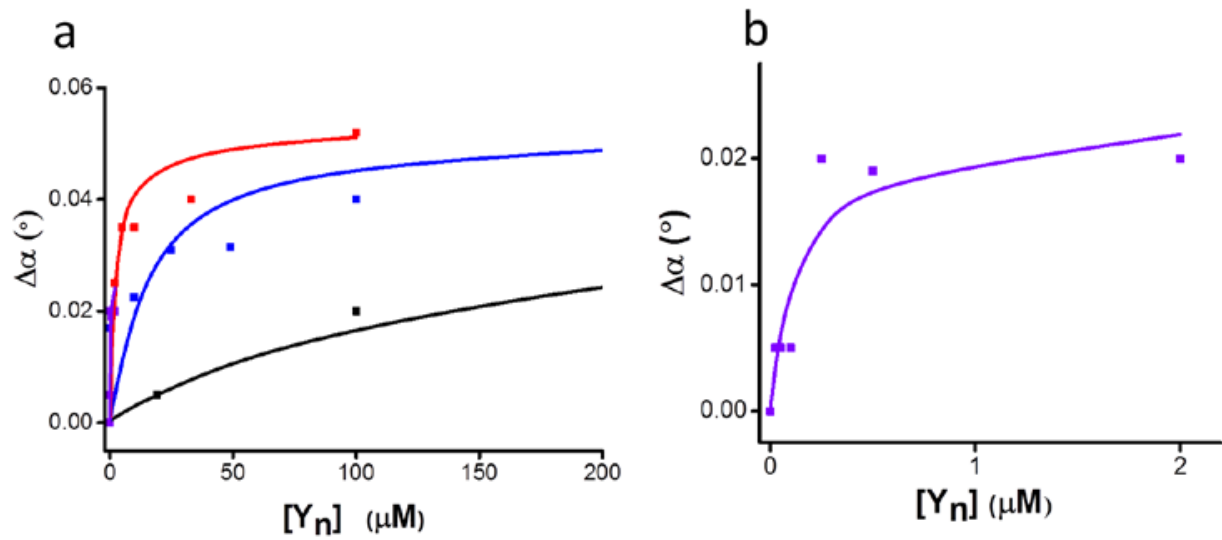

Figure 3.3 - Changes of the SPR coupling angle (markers) and Langmuir fits (curves) for the binding of the a) $\mathrm{Y}_{3}$ (black), $\mathrm{Y}_{4}$ (blue), $\mathrm{Y}_{5}$ (red) and b) $\mathrm{Y}_{6}$ (violet) peptides to a $\beta$-CD SAM.

\begin{tabular}{|c|c|}
\hline Peptide & $K_{L M}\left(10^{4} \mathrm{M}^{-1}\right)$ \\
\hline $\mathrm{Y}_{3}$ & 0.58 \\
\hline $\mathrm{Y}_{4}$ & 6.8 \\
\hline $\mathrm{Y}_{5}$ & 43 \\
\hline $\mathrm{Y}_{6}$ & 707 \\
\hline
\end{tabular}

Table 3.2 - Langmuir affinity constants $\left(K_{L M}\right)$ calculated from fitting the SPR data of the binding of each peptide to the $\beta$-CD SAM (Figure 3.3) to a 1:1 Langmuir binding model.

We fitted this set of data to a simple Langmuir model (Table 3.2), to estimate changes in binding affinity $\left(K_{L M}\right)$ upon changing the number of tyrosines in the peptide. We observed that, as the number of tyrosines in the peptide increases, the peptide concentration required for reaching the saturation level decreases, indicating that the higher valent peptides have a higher affinity, which is in line with the expectation for these multivalent systems. This is confirmed by the $K_{L M}$ values given in Table 3.2, which show an increase of affinity of about 3 orders over magnitude when changing the peptide valency from 3 to 6 . 


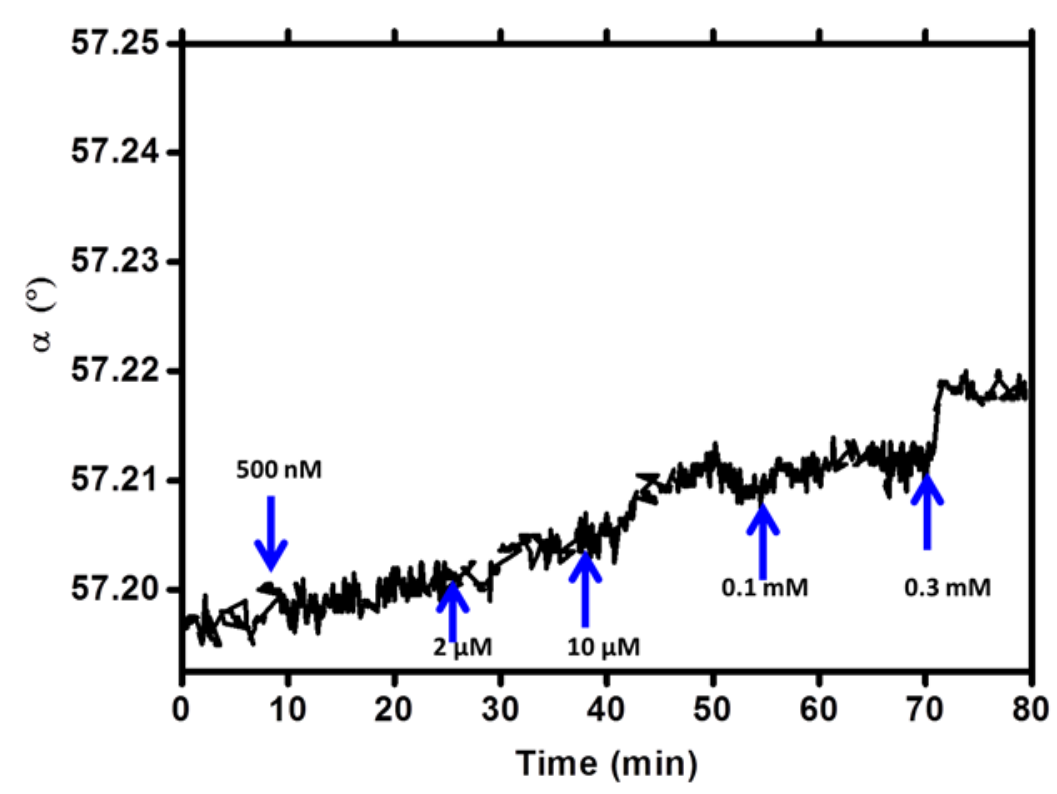

Figure 3.4 - SPR titration of the control peptide Ac(SGGSGGS) $)_{5}$ at a $\beta$-CD SAM.

To verify whether the amino acids Ser and Gly give rise to non-specific interactions, the peptide Ac(SGGSGGS) $)_{5}$ was synthesized, which represents a similar molecular structure as that of the pentavalent guest molecule Ac(SGGYGGS) $)_{5}\left(\mathrm{Y}_{5}\right)$ but each Tyr residue is replaced by a Ser residue. Afterwards the SPR response when flowing this control peptide over the $\beta$-CD SAM was measured (Figure 3.4). The experiment showed very little SPR response indicating that no binding of the control peptide to the $\beta$-CD SAM occurred, up to a $0.3 \mathrm{mM}$ peptide concentration. The control experiment presented a clear contrast to the binding of the pentavalent guest Ac(SGGYGGS) $)_{5}$, which showed a clear change of the SPR coupling angle already at a concentration of $0.5 \mu \mathrm{M}$. This result indicates that the Tyr residue is essential for the binding motif, and that the spacers have a negligible contribution to the overall affinity of the peptide with the $\beta$-CD SAM. 


\subsubsection{Linear free energy relationship}

A linear enhancement in binding free energy (as expressed by $\log K_{L M}$ ) was observed after plotting the $\log K_{L M}$ values against the valency (number of Y units) of the peptides as shown in Figure 3.5. This observation suggests that all tyrosines in the peptides are contributing to the overall binding affinity. To further probe the empirical relationship between $\log K_{L M}$ and the valency $n_{Y}$, the data was fitted to a linear free energy relationship (LFER), using $\log K_{L M}=\mathrm{C}+\beta \cdot n_{Y}$, where $\mathrm{C}$ is the intercept (with the $\mathrm{y}$ axis) and $\beta$ is the slope. From the linear fit, a $C$ value of 1.22 and a $\beta$ value of 0.85 were obtained.

An intrinsic binding constant at the surface of $120 \mathrm{M}^{-1}$ for a single interaction was derived, as is directly visible from the crossing point of the fitted line at a valency of 1 (monovalent tyrosine). This value is in good agreement with the reported binding constant $\left(120 \mathrm{M}^{-1}\right)$ for complexation of tyrosine with $\beta$-CD in solution. ${ }^{12}$ Moreover, the slope of the line (0.85) indicates that each additional tyrosine in the peptide leads to an enhancement of the binding affinity $\left(K_{L M}\right)$ by a factor 7 . This is a sign that the system is weakly multivalent. In such a system, the multivalency enhancement factor is moderate (1-10), meaning that each guest unit is for a significant part of the time in the unbound state and rapidly equilibrating between the bound and unbound states (for more detailed characteristics and implications of weakly multivalent system the reader is referred to Chapter 6). 


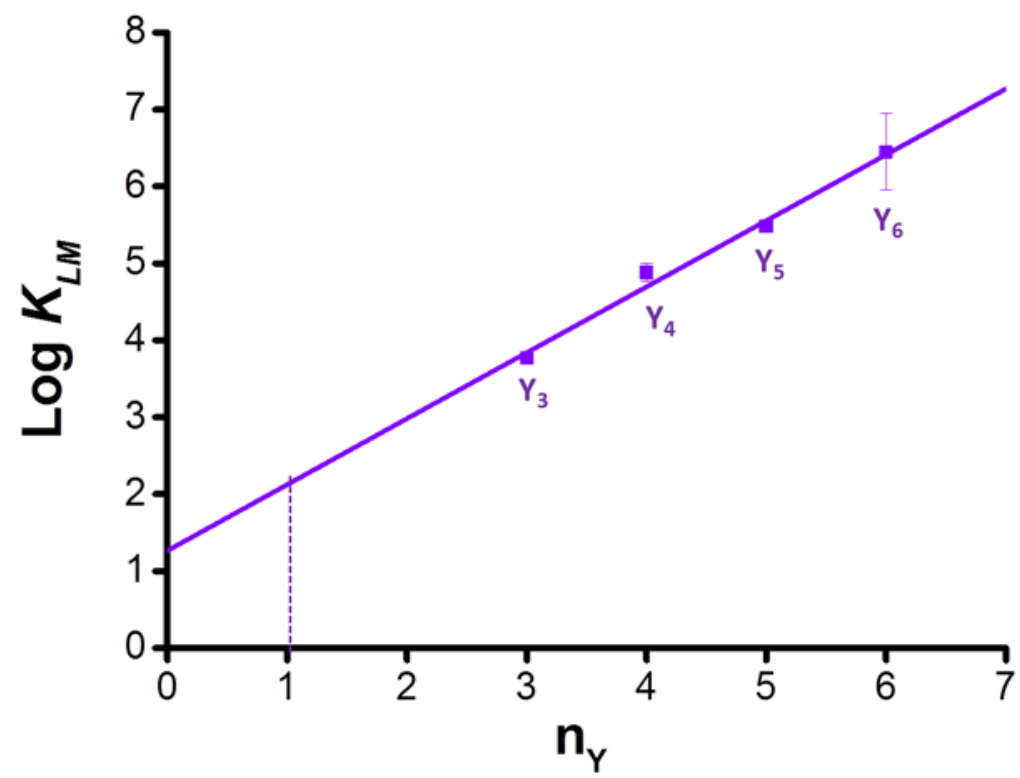

Figure 3.5 - Binding affinity values ( $\log K_{L M}$ ) obtained from the SPR titrations of the $\mathrm{Y}_{3}-\mathrm{Y}_{6}$ peptides adsorbing to $\beta$-CD SAMs, plotted against the number of tyrosines (valency, $n_{Y}$ ) in the peptide. The solid line represents a fit of the $\log K_{L M}$ values to a LFER $\left(\log K_{L M}=\mathrm{C}+\beta \cdot n_{Y}\right)$. The vertical dashed line at $n_{Y}=1$ crosses the LFER at the intrinsic affinity of a single Y- $\beta$-CD interacting pair. Error bars represent standard errors from two individual SPR experiments for $\mathrm{Y}_{4}$, $\mathrm{Y}_{5}$ and $\mathrm{Y}_{6}$ peptides.

\subsubsection{Multivalent binding model}

In order to make one generalized model to fit the SPR data of the binding of all peptides to the $\beta$-CD SAM, we plotted the experimental data vs. the peptide concentration and fitted the data to a multivalency model (Figure 3.6). The multivalent binding model is based on a full, explicit description of all (plausible) species involved in host-guest interactions at the $\beta$-CD SAM, as has been described before by our group. ${ }^{3}$ The model described here is an extension of that model as it incorporates the linear nature of the peptides used here. The model starts with the intermolecular binding event of first guest (tyrosine), followed by intramolecular steps for the subsequent guest units. Only the solution phase binding constant between tyrosine and $\beta-C D$ is included as the 
monovalent, intrinsic binding constant $\left(K_{i}\right)$ of the peptide, and an effective molarity (EM) value is included to provide the probability of intramolecular ring closure, as reported previously in the multivalent surface binding model for an adamantane guest system. ${ }^{3,12}$ An inverse relationship between $E M$ and the spacing between bound guest units is assumed here. In other words, the model assumes $E M_{i j}=E M_{0} /(j-i)$, where $j>i$, for species for which no sites are bound to the surface between Tyr sites $i$ and $j . E M_{0}$ is equal to $E M_{\max }$ at low coverage, but scales with the fraction of free surface $\beta$-CD sites $\theta_{f}$, so $E M_{i j}=\left(E M_{\max } \times \theta_{f}\right) /(j-i)$. Overall, all data for all peptides can thus be fitted simultaneously, yielding a $K_{i}$ and an $E M_{\max }$ value as the fit parameters. Fitting of the SPR data with the Langmuir and multivalency models showed similar correlation coefficients suggesting that both the models fit the data equally well. Graphically, a difference can be observed: the multivalency model keeps rising, following the data in a more gradual rise of the signal (Figure 3.6), whereas the Langmuir fit tends to level off sharper to a plateau (Figure 3.3).

We tried to optimize the fitting of the data with the multivalency model by testing various scenarios for fixing or changing the $K_{i}$ and $E M_{\max }$ values. Leaving both parameters free led to $K_{i}$ and $E M_{\max }$ values of $102 \mathrm{M}^{-1}$ and $58 \mathrm{mM}$, respectively, yet several other scenarios gave comparable overall errors. A clear compensating relationship between $K_{i}$ and $E M_{\max }$ was observed, i.e., similar quality fits could be obtained by fixing $K_{i}$ to a higher value and obtaining a lower $E M_{\max }$ value as the fit parameter. This is understandable, as the observed (qualitative and quantitative) upward trend with increasing valency means that the multivalency enhancement factor, which can also be written as the product of $K_{i} \times E M$ (with $E M=E M_{\max }$ ), cannot change a lot. ${ }^{3}$ A good scenario therefore was fixing the $K_{i}$ value to that known from literature $\left(120 \mathrm{M}^{-}\right.$ $\left.{ }^{1}\right)$ and optimizing the effective molarity value. The resulting effective molarity value was $46 \mathrm{mM}$, which is close to values observed before for the multivalent binding at $\beta$ CD SAMs. ${ }^{3}$ This corresponds to a $K_{i} \times E M$ value of 6 , which compares well to the multivalent enhancement factor (7) obtained from the slope found of the LFER, as discussed above. 

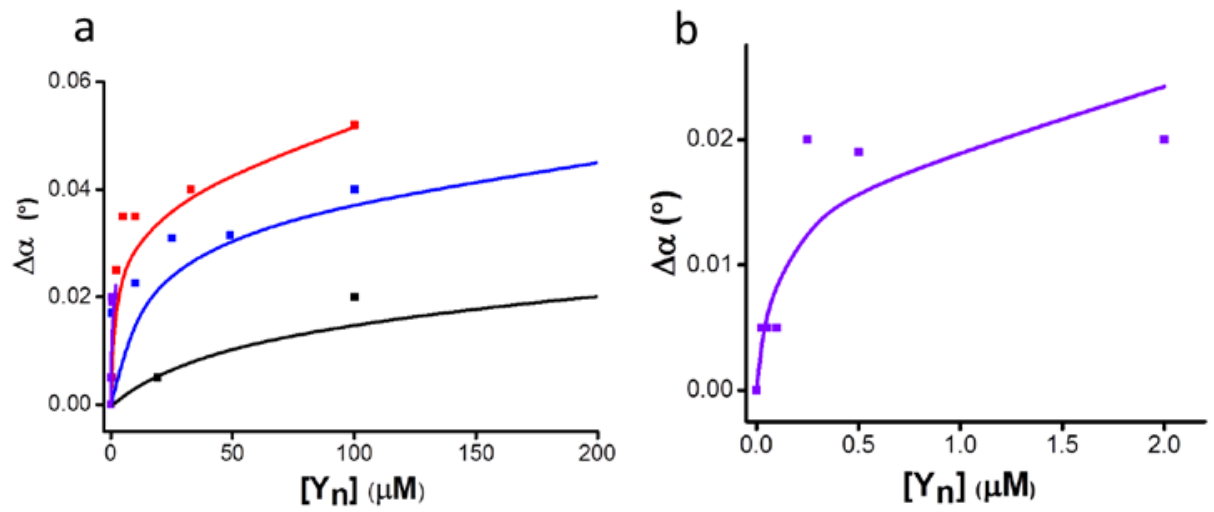

Figure 3.6 - Changes of the SPR coupling angle (markers) and multivalency model fits (curves) for the binding of the a) $\mathrm{Y}_{3}$ (black), $\mathrm{Y}_{4}$ (blue), $\mathrm{Y}_{5}$ (red) and b) $\mathrm{Y}_{6}$ (violet) peptides to a $\beta$-CD SAM.

\begin{tabular}{|c|c|c|}
\hline Peptide & $\begin{array}{c}K_{\text {ov }}{ }^{a} \\
\left(10^{4} \mathrm{M}^{-1}\right)\end{array}$ & $\begin{array}{c}K_{\text {ov }}{ }^{b} \\
\left(10^{4} \mathrm{M}^{-1}\right) \\
\text { (all species) }\end{array}$ \\
\hline$Y_{3}$ & 0.36 & 0.60 \\
\hline$Y_{4}$ & 2.0 & 4.0 \\
\hline$Y_{5}$ & 11 & 26 \\
\hline$Y_{6}$ & 62 & 169 \\
\hline \multicolumn{2}{|c|}{${ }^{\mathrm{a}} K_{o v}$ calculated according to equation 3.1} \\
${ }^{\mathrm{b}} K_{o v}$ calculated according to equation 3.2
\end{tabular}

Table 3.3 - Table showing the overall binding constants $\left(K_{o v}\right)$ calculated from 1:1 Langmuir and multivalency binding models respectively. $K_{o v}$ is calculated separately for fully bound peptide and all species (fully and partially bound).

In order to compare the Langmuir and multivalency fits more quantitatively, the multivalency fit parameters need to be translated in overall affinities, $K_{o v}$. For strong multivalent systems, the overall affinity is given by: ${ }^{3}$ 


$$
K_{o v}=K_{i}\left(K_{i} \times E M\right)^{n-1}
$$

This equation assumes that the species that binds with all sites to the surface is the main surface species, which is true for strong multivalent systems (with $K_{i} \times E M>10$ ) like the adamantane system studied before. However, in case of the peptides studied here, also lower valent species contribute to the binding, and therefore this equation can best be transformed into:

$$
\mathrm{K}_{o v}=\sum_{j=1}^{n}\left(\begin{array}{c}
n \\
j
\end{array}\right) K_{i}\left(K_{i} \times E M\right)^{j-1}
$$

Table 3.3 gives the $K_{o v}$ values as calculated from both equations. The higher values provided by equation 3.2 indicate that incorporating the lower valent species is indeed important. The $K_{o v}$ values calculated from incorporating all plausible bound species are comparable to the $K_{L M}$ values, and they show a similar enhancement factor (7) as found for the LFER. More importantly, the weakly multivalent nature of the peptide guests suggests that even at low peptide concentrations (where $E M=E M_{\max }$ ) the peptide exhibits a broad population of surface species with different valencies of interaction with the surface (see also details explained in Chapter 6). ${ }^{16}$

\subsubsection{Influence of molecular geometry on binding affinity}

To evaluate the effect of changing the distance between neighboring $\mathrm{Y}$ units, the tetravalent peptides $\mathrm{Ac}(\mathrm{SGYGS})_{4}, \mathrm{Ac}(\mathrm{SGY})_{4}$, and AcSYYGYYS were synthesized, and their binding to $\beta$-CD SAMs was evaluated and compared to $\mathrm{Y}_{4}$. In the case of $\mathrm{Ac}(\mathrm{SGYGS})_{4}\left(K_{L M}=6.3 \times 10^{4} \mathrm{M}^{-1}\right)$ and $\mathrm{Ac}(\mathrm{SGY})_{4}\left(K_{L M}=5.8 \times 10^{4} \mathrm{M}^{-1}\right)$ no significant difference in binding affinity compared to $\mathrm{Y}_{4}\left(K_{L M}=5.7 \times 10^{4} \mathrm{M}^{-1}\right)$ was observed. These sequences correspond to Y-Y distances of 19-23 $\AA$ and 11-13 $\AA$, respectively. When compared to the spacing of $\beta$-CD cavities of $21 \AA$ observed before, ${ }^{3}$ in particular the high affinity of $\mathrm{Ac}(\mathrm{SGY})_{4}$ is remarkable. This might be attributed to the fact that although the center to center distance between two adjacent $\beta$-CD cavities in monolayer is $21 \AA$, the edges of the cavities in two adjacent $\beta$-CD molecules are separated by only $12 \AA$. Therefore it might be possible a for peptide to bridge and occupy neighboring $\beta-C D$ cavities. 
In the case of AcSYYGYYS, where two pairs of adjacent tyrosines are separated by one glycine, no specific interaction with the $\beta$-CD SAM was observed in the concentration range used (Figure 3.7). The SPR titration curves of interaction between the peptide and the $\beta-\mathrm{CD}$ and $\mathrm{EG}_{6}$ SAMs are shown in Figure. 3.7. On the $\mathrm{EG}_{6}$ surface, a similar coupling angle change as that on a $\beta$-CD SAM was observed. This might be due to very poor solubility of the peptide in buffer solution, thus in the change in coupling angle on both SAMs may arise from non-specific adsorption of the peptide to the SAM.

a

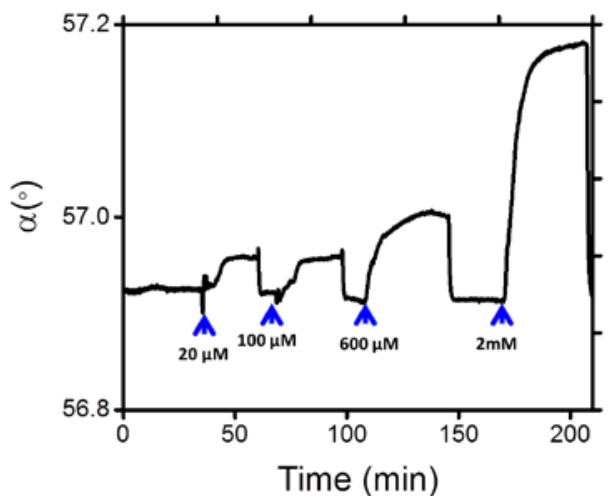

b

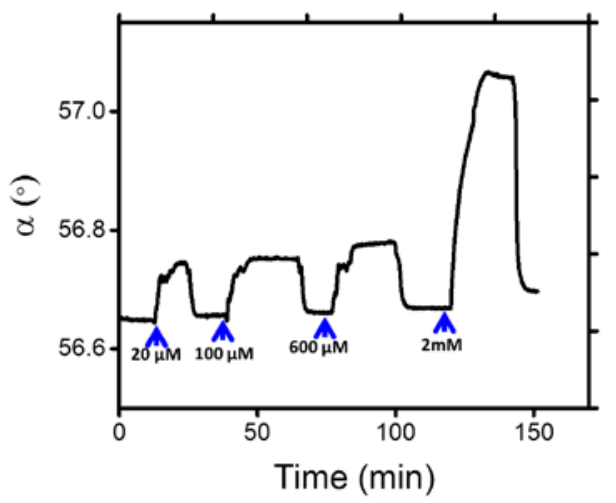

Figure 3.7 - SPR coupling angle time traces of AcSYYGYYS at a) $\beta-C D$ and b) EG ${ }_{6}$ SAMs as a function of peptide concentration.

Overall, these results indicate a small or negligible influence of linker length on the binding affinity of these peptides with the $\beta$-CD SAM as long as the aromatic units can interact with the host cavities. Only when the distance is really too short (as is the case for AcSYYGYYS), the affinity drops considerably. 

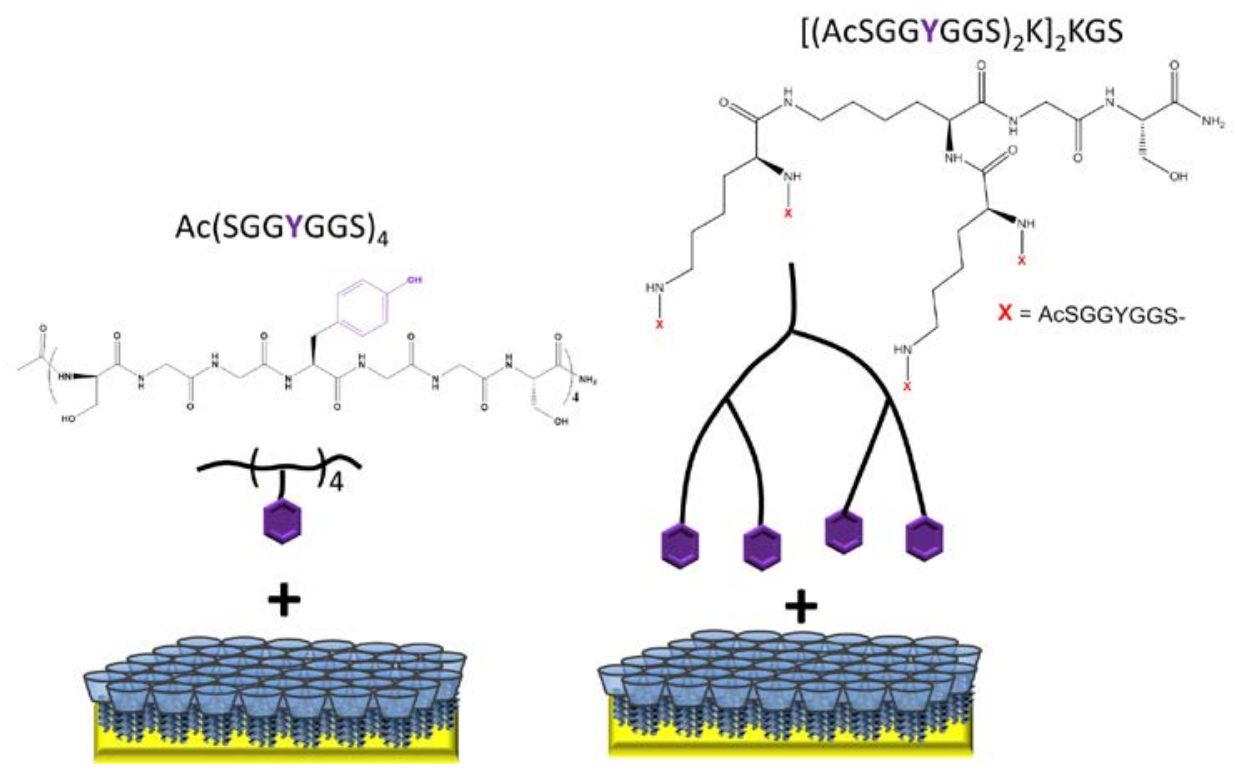

Figure 3.8 - Synthesized peptides with different molecular configurations (linear and branched).

In a next step, an attempt was made to verify the influence of molecular configuration on binding affinity. A branched guest peptide [(AcSGGYGGSS) $\left.{ }_{2} \mathrm{~K}\right]_{2} \mathrm{KGS}$ with four Tyr residues was synthesized (Figure 3.8). The Langmuir constant $\left(K_{L M}\right)$ calculated from fitting of the SPR data (Figure 3.9) was found to be $6.8 \times 10^{4} \mathrm{M}^{-1}$, which was very similar to the binding constant of its linear counterpart Ac(SGGYGGS $)_{4}\left(K_{L M}=5.7 \times\right.$ $\left.10^{4} \mathrm{M}^{-1}\right)$. Although the branched tetravalent guest [(AcSGGYGGSS $\left.)_{2} \mathrm{~K}\right]_{2} \mathrm{KGS}$ and the linear tetravalent guest differed in length and flexibility of the spacer as a result of the different molecular geometries, the distance between adjacent binding sites was long enough (52 $\AA$ ) to bind to adjacent $\beta$-CD receptors, resulting in similar binding constants with the $\beta$-CD SAM. Putting together these results suggests that, if the spacer in between the Tyr residues is long enough for them to bind to adjacent $\beta$-CD receptors on the surface, the overall binding constant is rather insensitive to length and configuration. 
a

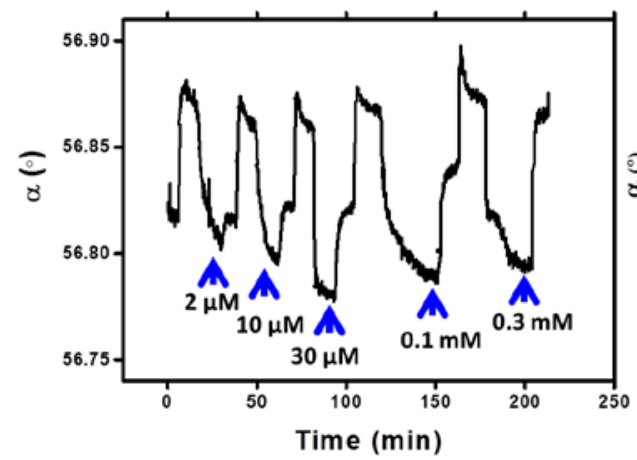

b

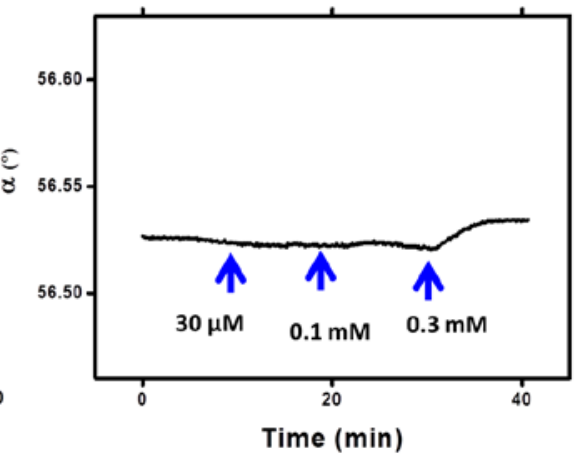

Figure 3.9 - SPR titration data of branched [(AcSGGYGGSS) $\left.{ }_{2} \mathrm{~K}\right]_{2} \mathrm{KGS}$ peptide on a) $\beta$-CD $\mathrm{SAM}$ b) $\mathrm{EG}_{6} \mathrm{SAM}$.

\subsection{Conclusions}

In conclusion, we have shown a well-defined and highly specific host-guest model system to study weak, peptide-based multivalent interactions at interfaces. We demonstrated that, by carefully designing the architecture of supramolecular peptide ligands with aromatic guest units as the interacting moieties, the multivalency of binding of these peptides to a $\beta$-CD surface can be exploited to tune their binding affinity with that surface. Both Langmuir and multivalency models can be fitted to the data, but the multivalency model provides deeper insight in the intrinsically weak nature of the interactions. Moreover it has been observed that if the optimum interligand spacing is maintained, ligands with different molecular geometries, i.e. linear and branched, can show similar binding affinities with the $\beta$-CD surface. This freedom of using supramolecular systems with branched and linear structures is further exploited in subsequent chapters to interrogate various physical and biological properties.

\subsection{Acknowledgements}

Dr. Qi An is gratefully acknowledged for her guidance in the synthesis of peptides and fruitful discussions. 


\subsection{Materials and methods}

General. Chemicals were obtained from commercial sources and used as received. Fmoc-Rink Amide MBHA resin (0.52 mmol/g), Fmoc-protected amino acids and related coupling agents for peptide synthesis were purchased from Multisyntech GmbH Chemicals. Hexa(ethylene glycol) methyl ether thiol $\left(\mathrm{EG}_{6}\right)$ was purchased from SigmaAldrich. Solvents employed in the purification of peptides were purchased from SigmaAldrich.

Equipment. JMS-T100LC mass spectrometer was used to determine the mass for the peptide. High-performance liquid chromatography (HPLC) experiments were performed with equipment from Waters. Surface plasmon resonance (SPR) experiment was conducted using $50 \mathrm{~nm}$ SPR gold substrates obtained from Ssens BV using equipment from Resonant probes $\mathrm{GmbH}$. The angle of minimum reflection was traced during experiments. The gold substrate with the monolayer was optically matched to the prism using an index matching oil. A flow cell was placed on the monolayer and was filled by target solutions using a syringe pump. The solutions of guest molecules were prepared with phosphate buffer. Phosphate buffered saline (PBS) or $5 \mathrm{mM}$ solution of $\beta$ cyclodextrin in PBS were used to wash the substrate after sample injection to establish the SPR baseline.

Synthesis. Peptides were synthesized with solid phase peptide synthesis with FmocRink Amide MBHA resin, following standard Fmoc protocols. Purification of the peptide was done with reversed phase HPLC. Preparative column (Waters 2535 quaternary gradient module with $\mathrm{XBridge}^{\mathrm{TM}}$ prep C18 $5 \mu \mathrm{m} \mathrm{OPD}^{\mathrm{TM}} 19 \times 250 \mathrm{~mm}$ preparative column)with eluents $0.1 \%$ aqueous TFA and $0.1 \%$ TFA in acetonitrile were used to purify crude peptide. The extent of purification was checked using an analytical HPLC (Waters 2535 quaternary gradient module XBridge C18 $5 \mu \mathrm{m}$ column with $4.6 \times 250 \mathrm{~mm}$ dimensions). Same eluents as that of preparative HPLC were used. HPLC retention times were observed following analytical HPLC with a solvent gradient of $0-90 \%$ acetonitrile over 90 min timescale. Mass of purified peak was determined using LC-MS (Waters 2535 module coupled to micromass LCT)). The quality of the peptides was checked by analytical HPLC and ESI mass spectra. Synthesis of heptakis- 
\{6-O-[12-(thiododecyl)dodecanoyl)]-2,3-di-O-methyl $\}$ - $\beta$-cyclodextrin and its assembly on gold surfaces was done as reported previously. ${ }^{17}$

\section{Ac(SGGYGGS) 3}

Peptide Ac(SGGYGGS) 3 was synthesized and purified, with a yield of $9.79 \mathrm{mg}, 5.7 \%$. ESI mass spectra of peptide Ac(SGGYGGS) ${ }_{3}$ confirmed the successful synthesis of the peptide. Chemical formula: $\mathrm{C}_{71} \mathrm{H}_{98} \mathrm{~N}_{22} \mathrm{O}_{31}$, Mw: 1754.7. [M+Na $\left.{ }^{+}\right]$: calculated: 1777.6; found: 1776.5; [M+2Na $\left.{ }^{+}\right] / 2$ : calculated: 900.3; found: 899.8. The analytical HPLC is given in Figure 3.10 .

\section{Ac(SGGYGGS) 4}

Peptide Ac(SGGYGGS) ${ }_{4}$ was synthesized and purified, with a yield of $28 \mathrm{mg}, 12.4 \%$. ESI mass spectra of peptide Ac(SGGYGGS) ${ }_{4}$ confirmed the successful synthesis of the peptide. Chemical formula: $\mathrm{C}_{94} \mathrm{H}_{129} \mathrm{~N}_{29} \mathrm{O}_{41}$, Mw: 2319.89. [M+2 $\left.\mathrm{H}^{+}\right] / 2$ : calculated: 1161.0; found: 1161.3; [M+3H $\left.\mathrm{H}^{+}\right] / 3$ : calculated: 773.3; found: 774.1. The analytical HPLC is given in Figure 3.10 .

\section{Ac(SGGYGGS)}

Peptide Ac(SGGYGGS) 5 was synthesized and purified, with a yield of $25 \mathrm{mg}, 8.6 \%$. ESI mass spectra of peptide Ac(SGGYGGS) $)_{5}$ confirmed the successful synthesis of the peptide. Chemical formula: $\mathrm{C}_{117} \mathrm{H}_{160} \mathrm{~N}_{36} \mathrm{O}_{51}, \mathrm{Mw}$ : 2885.10. [M+2 $\left.\mathrm{H}^{+}\right] / 2$ : calculated 1443.6, found: 1444.1; $\left[\mathrm{M}+3 \mathrm{H}^{+}\right] / 3$ : calculated: 962.7, found: 963.3 . The analytical HPLC is given in Figure 3.10.

\section{Ac(SGGYGGS) 6}

Peptide Ac(SGGYGGS) 6 was synthesized and purified, with a yield of 5.4\%. ESI mass spectra of peptide Ac(SGGYGGS) ${ }_{6}$ confirmed the successful synthesis of the peptide. Chemical formula: $\mathrm{C}_{140} \mathrm{H}_{191} \mathrm{~N}_{43} \mathrm{O}_{61}$, Exact mass: 3450.32. $\left[\mathrm{M}+2 \mathrm{Na}^{+}\right] / 2$ : calculated 1748.1, found: 1748.3 ; $\left[\mathrm{M}+3 \mathrm{Na}^{+}\right] / 3$ : calculated: 1173.1 , found: 1173.2 . The analytical HPLC is given in Figure 3.10. 


\section{Ac(SGGSGGS)}

Peptide Ac(SGGSGGS) $)_{5}$ was synthesized and purified, with a yield of $6.0 \%$. ESI mass spectra of peptide Ac(SGGSGGS) ${ }_{5}$ confirmed the successful synthesis of the peptide. Chemical formula: $\mathrm{C}_{87} \mathrm{H}_{140} \mathrm{~N}_{36} \mathrm{O}_{51}$, Exact mass: 2504.95. $\left[\mathrm{M}+\mathrm{Na}^{+}+\mathrm{H}^{+}\right] / 2$ : calculated 1264.5, found: 1264.2; [M+2 $\left.\mathrm{H}^{+}\right] / 2$ : calculated: 1253.5, found: 1253.1 . The molecular structure of the peptide is shown in Figure. 3.10

\section{6. $\left[(\mathrm{AcSGGYGGS})_{2} \mathrm{~K}\right]_{2} \mathrm{KGS}$}

The branched peptide $\left[\left(\text { AcSGGYGGSS) }{ }_{2} \mathrm{~K}\right]_{2} \mathrm{KGS}\right.$ (is obtained after purification, with a yield of $5.5 \%$ (5.7 mg). The ESI-MS spectra confirmed the successful synthesis of the peptide. Chemical formula: $\mathrm{C}_{135} \mathrm{H}_{199} \mathrm{~N}_{41} \mathrm{O}_{58}$. Exact mass: 3322.39. $\left[\mathrm{M}+2 \mathrm{Na}^{+}\right] / 2$ : calculated: 1684.2; found: 1684.4. $\left[\mathrm{M}+3 \mathrm{H}^{+}\right] / 3$ : calculated: 1108.5; found:1108.7. The analytical HPLC is given in Figure 3.10.

\section{Ac(SGY) 4}

Peptide $\mathrm{Ac}(\mathrm{SGY})_{4}$ was synthesized and purified, with a yield of $14.0 \%$. ESI mass spectra of peptide $\mathrm{Ac}(\mathrm{SGY})_{4}$ confirmed the successful synthesis of the peptide. Chemical formula: $\mathrm{C}_{56} \mathrm{H}_{70} \mathrm{~N}_{12} \mathrm{O}_{21}$, Exact mass: 1288.49. Found: 1288.65 ; $\left[\mathrm{M}+2 \mathrm{H}^{+}\right] / 2$ : calculated: 645.24 , found: 644.40

\section{Ac(SYYGYYS)}

Peptide Ac(SYYGYYS) is synthesized and purified, with a yield of 12.0\%. ESI mass spectra of peptide Ac(SYYGYYS) confirmed the successful synthesis of the peptide. Chemical formula: $\mathrm{C}_{44} \mathrm{H}_{51} \mathrm{~N}_{7} \mathrm{O}_{14}$, Exact mass: 943.95. Found: 943.58. The analytical HPLC showed a single peak, indicating product of high purity (>90\%). 

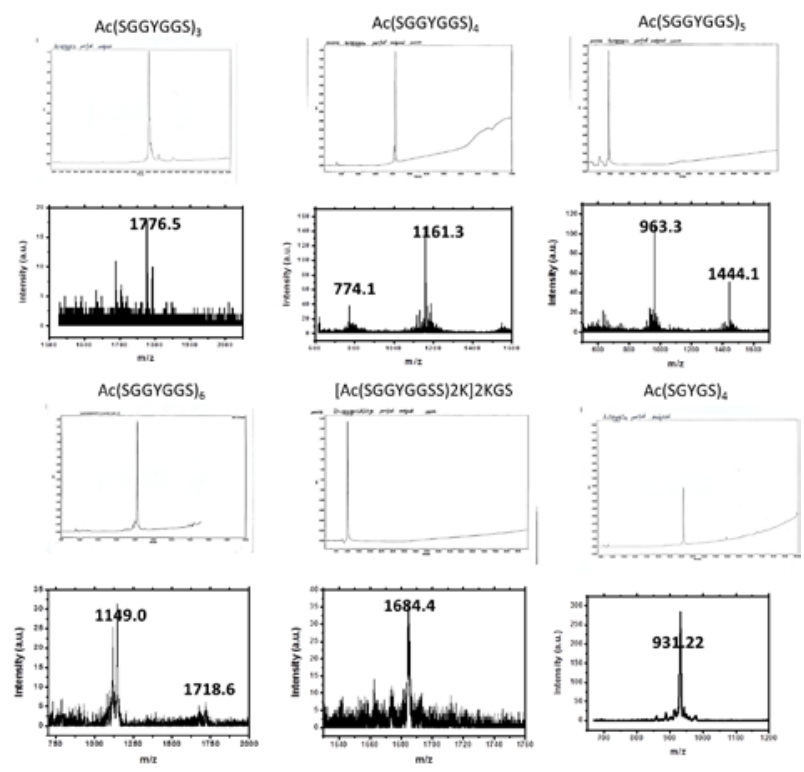

Figure 3.10 - HPLC and mass traces of purified peptides

\subsection{References}

1. Mammen, M. et al. Polyvalent interactions in biological systems: implications for design and use of multivalent ligands and inhibitors. Angewandte Chemie International Edition 37, 2754-2794 (1998).

2. Huskens, J. et al. Attachment of molecules at a molecular printboard by multiple host-guest interactions. Angewandte Chemie International Edition 41, 4467-4471 (2002).

3. Huskens, J. et al. A Model for describing the thermodynamics of multivalent host-guest interactions at interfaces. Journal of the American Chemical Society 126, 6784-6797 (2004).

4. Yang, L. et al. Reversible and oriented immobilization of ferrocene-modified proteins. Journal of the American Chemical Society 134, 19199-19206 (2012).

5. Nijhuis, C.A. et al. Multivalent dendrimers at molecular printboards: influence of dendrimer structure on binding strength and stoichiometry and their electrochemically induced desorption. Langmuir 21, 7866-7876 (2005). 
6. Nijhuis, C.A. et. al. Binding control and stoichiometry of ferrocenyl dendrimers at a molecular printboard. Journal of the American Chemical Society 126, 12266-12267 (2004).

7. Martinez-Veracoechea, F.J. et al. Designing super selectivity in multivalent nano-particle binding. Proceedings of the National Academy of Sciences 108, 10963-10968 (2011).

8. Carlson, C.B. et al. Selective tumor cell targeting using low-affinity, multivalent interactions. ACS Chemical Biology 2, 119-127 (2007).

9. Del Valle, E.M.M. Cyclodextrins and their uses: a review. Process Biochemistry 39, 1033-1046 (2004).

10. Crespo-Biel, O. et al. Writing with molecules on molecular printboards. Dalton Transactions, 2737-2741 (2006).

11. Amblard, M. et al. Methods and protocols of modern solid phase peptide synthesis. Molecular Biotechnology 33, 239-254 (2006).

12. Rekharsky, M. et al. Chiral recognition thermodynamics of $\beta$-cyclodextrin: the thermodynamic origin of enantioselectivity and the enthalpy-entropy compensation effect. Journal of the American Chemical Society 122, 44184435 (2000).

13. Beulen, M.W.J. et al. Host-guest interactions at self-assembled monolayers of cyclodextrins on gold. Chemistry - A European Journal 6, 1176-1183 (2000).

14. Stenberg, E. et al. Quantitative determination of surface concentration of protein with surface plasmon resonance using radiolabeled proteins. Journal of Colloid and Interface Science 143, 513-526 (1991).

15. Quinn, J.G. et al. Development and application of surface plasmon resonancebased biosensors for the detection of cell-ligand interactions. Analytical Biochemistry 281, 135-143 (2000).

16. Perl, A. et al. Gradient-driven motion of multivalent ligand molecules along a surface functionalized with multiple receptors. Nature Chemistry 3, 317-322 (2011).

17. Beulen, M.W.J. et al. Self-assembled monolayers of heptapodant $\beta$ cyclodextrins on gold. Langmuir 14, 6424-6429 (1998). 


\title{
Chapter 4
}

\section{Dual functional EYPD peptide conjugates facilitate binding to $\beta-C D$ surfaces and $\alpha_{9} \beta_{1}$ integrins}

\begin{abstract}
In this chapter the programmable multivalency concept was exploited to immobilize bioactive peptides on the $\beta$-cyclodextrin ( $\beta$-CD) self-assembled monolayer (SAM). A peptide (IFQEYPD) containing two different types of aromatic amino acids, i.e., tyrosine (Y) and phenylalanine (F) which have different intrinsic binding affinities with $\beta$-CD, was developed. The contributions of both aromatic amino acids to the overall binding affinity of the peptide to the $\beta$-CD SAM were evaluated. This peptide was coupled to the terminal functional groups of PAMAM dendrimers. Due to the globular nature of the PAMAM dendrimers, not all peptide moieties were able to interact with the $\beta$-CD surface. Using quantitative measurements of the binding affinities and by theoretical modeling, the numbers of peptide moieties interacting with the $\beta$-CD surface were quantified for a range of dendrimers. From this calculation the density of free peptides, i.e., those not interacting with the $\beta$-CD surface, was determined. This free peptide density is an important control parameter in the study of integrin clustering in cells to trigger growth factor activity as described in Chapter 5.
\end{abstract}




\subsection{Introduction}

Supramolecular host-guest chemistry provides an elegant way to generate surfaces to gain spatiotemporal control over bioactivity. ${ }^{1}$ Host-guest chemistry has wide applications in immobilizing various proteins, peptides and growth factors. ${ }^{2,3} \beta-\mathrm{CD}$ surfaces are appealing as host surfaces since they form a well-defined uniform monolayer on different solid surfaces. ${ }^{4,5}$ Various guest molecules can interact with $\beta$ $\mathrm{CD}$ surfaces to display dynamically bioactive ligands to trigger specific cell responses. ${ }^{2,6}$ We showed in the Chapter 3 that by controlling the number of host-guest interactions between multivalent peptide ligands and the $\beta$-CD surface, the binding affinity of these peptide ligands with the surface can be tuned.

Thus we envisioned that the conjugation of a bioactive peptide to the periphery of a PAMAM dendrimer can serve a dual functional role of binding to a $\beta$-CD surface as well as cell surface receptors. A previous study on other guest-modified dendrimers has indicated that ligand sites coupled to the periphery of a PAMAM dendrimer cannot interact all simultaneously with the $\beta$-CD surface, due to the globular nature of these dendrimers. ${ }^{7}$ Moreover, by understanding the multivalent host-guest binding behavior of the dendrimers in quantitative detail, it is possible to calculate the number of ligands interacting with the $\beta$-CD surface. ${ }^{8}$ When applied to peptide-functionalized dendrimers, these results imply that peptides that remain unbound to the $\beta$-CD surface would be available for interacting with cells and that the surface density of them can be tuned rationally.

As a bioactive peptide sequence we selected IFQEYPD as EYPD has been reported to be specific for binding to transmembrane $\alpha_{9} \beta_{1}$ integrin receptors known to induce cell migration and vascular endothelial growth factor (VEGF) activation. ${ }^{9}$ Moreover, this peptide contains a phenylalanine $(\mathrm{F})$ and a tyrosine $(\mathrm{Y})$ unit in its sequence which are known to form host-guest complexes with $\beta$-CD. ${ }^{10}$ In Chapter 3, we have shown that variation of the number of $\mathrm{Y}$ units in the peptide leads to a programmable overall affinity of the peptide in binding to the $\beta$-CD surface. In this chapter we discuss the influence of different numbers of $\mathrm{F}$ and $\mathrm{Y}$ in bioactive peptides on their binding affinity with the $\beta$-CD surface. We used the binding affinity data of the linear peptides and of peptide-conjugated dendrimers to estimate the surface binding valency of the globular 
dendrimers. This binding valency is then used to determine the density of free peptides (exposed ligand density), i.e., the peptides that are not interacting with the $\beta$-CD SAM. The surface coverage of dendrimers is expressed as free peptide per surface area and used to calculate the exposed ligand density per surface area. This exposed ligand density is used subsequently to control integrin signaling as described in Chapter 5.

\subsection{Results and discussion}

Figure 4.1 shows the linear bioactive peptide variants (IFQEYPD, GYGIFQEYPD) and their conjugates with PAMAM dendrimers (Figure 4.1). The peptides contains F and Y amino acids which are known to interact with a $\beta$-CD SAM by host-guest interactions. When coupled to the end groups of PAMAM dendrimers, some peptide moieties will interact with the $\beta$-CD SAM while others remain available to interact with integrin receptors. The dendrimer generation was varied to synthesize peptide dendrimer conjugates with different binding valencies, to provide control over the exposed ligand density (Figure 4.1c).

a)

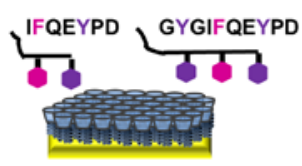

c)

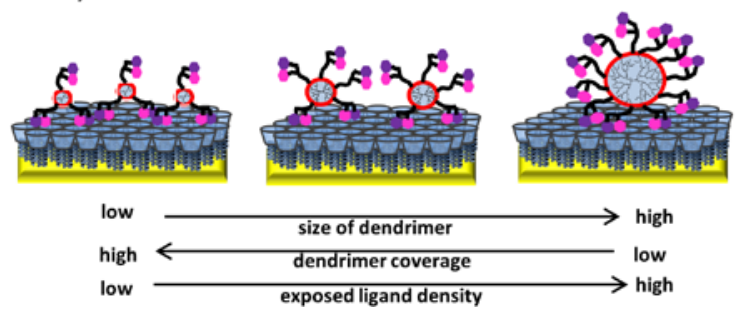

b)

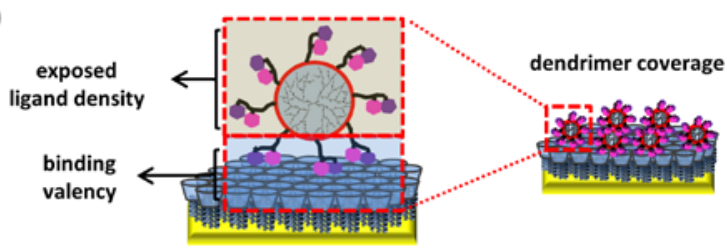

d)

$$
\begin{aligned}
& \text { AcSGGYGIFQEYPD, } Y_{2} F_{1} \\
& \text { Ac(IFQEYPD) })_{2}, Y_{2} F_{2} \\
& \text { Ac(IFQEYPD) })_{2} I F Q, Y_{2} F_{3} \\
& \text { Ac(IFQEYPD) })_{3}, Y_{3} F_{3} \\
& \text { AC(IYQEYPD) })_{2}, Y_{4} F_{0}
\end{aligned}
$$

Figure 4.1 - Schematic overview of IFQEYPD peptides and their dendrimer conjugates with tunable exposed ligand densities at a $\beta$-CD SAM. a) Molecular structures of linear peptides used to establish correlation between binding affinity and valency. b) Concept showing immobilization of dual functional dendrimers on $\beta$-CD SAM. c) Cartoon showing dependence of exposed ligand density on various molecular parameters. d) Linear peptides studied here to assess the 
contributions of the phenylalanine $(\mathrm{F})$ and tyrosine $(\mathrm{Y})$ units to the binding of the peptides to the $\beta$-CD SAM.

\subsubsection{Contribution from phenylalanine to the binding of a bioactive peptide to $\beta$ - CD surface}

In Chapter 3, we have investigated the effect of a valency of three to six tyrosine (Y) residues in a linear peptide on the binding affinity with the $\beta$-CD surface. We found that a stepwise increase in the valency of $\mathrm{Y}$ residues in a peptide led to an increase of the binding affinity with the $\beta$-CD surface by approximately a factor of 7 . When compared to other aromatic amino acids, tyrosine forms the strongest host-guest complex with $\beta$ $\mathrm{CD}\left(120 \mathrm{M}^{-1}\right) .^{10}$ The weaker binding phenylalanine $\left(60 \mathrm{M}^{-1}\right)^{10}$ is also contained in the sequence of the bioactive peptides targeted here, and its contribution to the overall binding affinity was studied first.

The linear peptides with $\mathrm{Y}$ and $\mathrm{F}$ are shown in Figure 4.1d. The separation between tyrosine and phenylalanine in these sequences is estimated to be $14 \AA$, which is sufficient to allow binding to two adjacent $\beta$-CD cavities as found experimentally for $\mathrm{Y}$ containing peptides in Chapter 3.

a

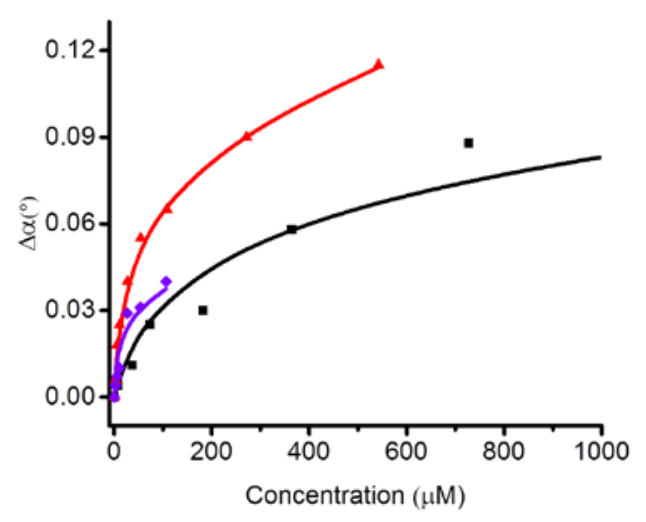

b

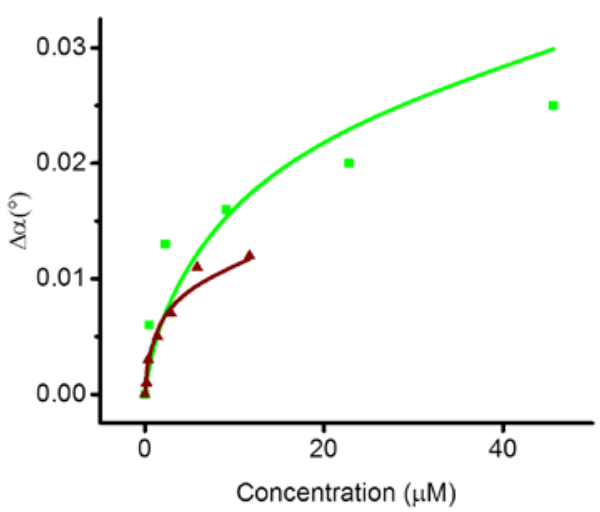

Figure 4.2 - SPR angle change, $\Delta \alpha$, for all linear peptides as a function of the peptide concentration, fitted to the multivalency model: $\mathrm{Y}_{2} \mathrm{~F}_{1}$ (black), $\mathrm{Y}_{2} \mathrm{~F}_{2}$ (red), and $\mathrm{Y}_{4} \mathrm{~F}_{0}$ (purple). b) $\mathrm{Y}_{2} \mathrm{~F}_{3}$ (green) and $\mathrm{Y}_{3} \mathrm{~F}_{3}$ (brown). 
After the synthesis of the various bioactive peptide variants, their binding affinity with the $\beta$-CD surface was quantified using SPR. The change in coupling angle $(\Delta \alpha)$ was plotted (Figure 4.2) against the concentration of each peptide and Langmuir fits (not shown) were used to obtain affinity constants $K_{L M}$ following the methodology described in Chapter 3. When plotting the $K_{L M}$ values for the series of peptides with $2 \mathrm{Y}$ units and 1-3 F units vs the valency, here defined as the total number of $\mathrm{Y}$ and $\mathrm{F}$ units together, a linear trend is observed (Figure 4.3). The slope of the trend line is clearly less than that of the pure tyrosine system (Chapter 3).

These results indicate: (i) a clear influence of phenylalanine on the binding affinity, as every additional $\mathrm{F}$ leads to an increase of $K_{L M}$ of about a factor 4 (slope: $\Delta \log K_{L M}=$ 0.6), and (ii) the slope for $F$ is lower than that of $Y$ signifying the difference in individual binding affinity $K_{i}$ between $\mathrm{Y}$ and $\mathrm{F}$.

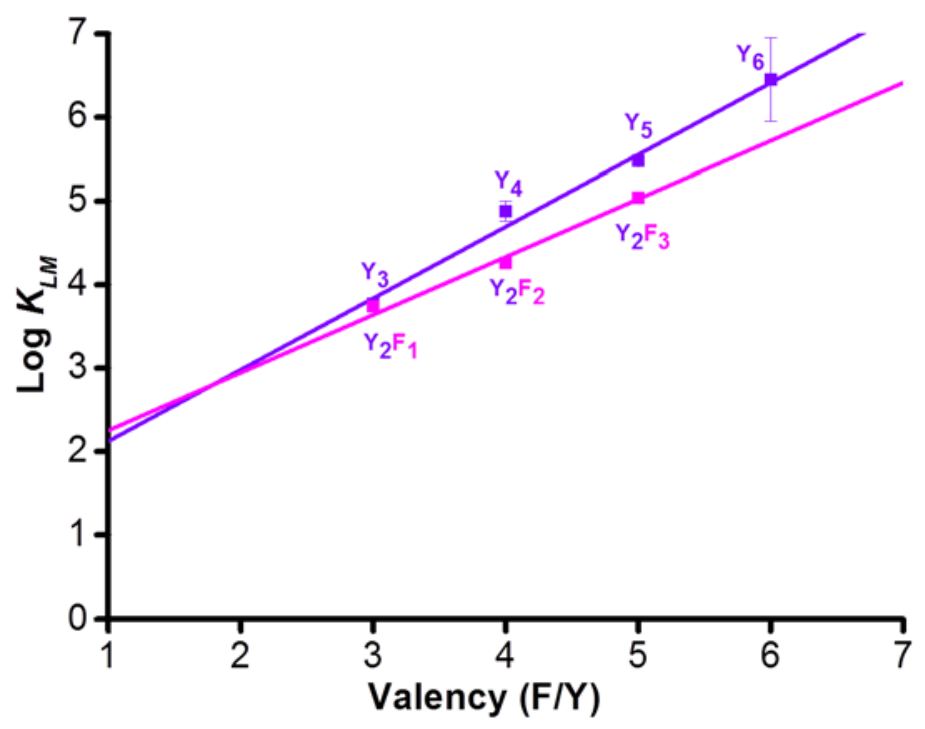

Figure 4.3 - Binding affinities $\left(\log K_{L M}\right.$ ) of the bioactive peptides with $2 \mathrm{Y}$ and 1-3 F units calculated using Langmuir fitting of SPR titration data plotted vs. the valency (total number of aromatic amino acids in the peptides). The pink colored line corresponds to a linear fit of the mixed F/Y peptides, while the purple markers and trend line corresponds to the $\log K_{L M}$ values of the pure $\mathrm{Y}$ system $\left(\mathrm{Y}_{3}-\mathrm{Y}_{6}\right.$, see Chapter 3$)$. 
As explained in Chapter 3, we extended this methodology to determine a linear free energy relationship (LFER), using the following equation:

$$
\log K_{L M, \text { calc }}=n_{Y} \cdot a_{Y}+n_{F} \cdot a_{F}+\mathrm{C}
$$

where $n$ indicates the number of aromatic amino acid residues ( $\mathrm{Y}$ or $\mathrm{F}$ ) and $a$ the dependence of $\log K_{L M}$ on these $\mathrm{Y}$ and $\mathrm{F}$ valencies. When using all $K_{L M}$ data shown in Figure 4.3, and also include the peptides $\mathrm{Y}_{3} \mathrm{~F}_{3}$ and $\mathrm{Y}_{4} \mathrm{~F}_{0}$ (see Figure 4.1d), we found an optimal fit for $a_{Y}=0.86, a_{F}=0.69$, and $\mathrm{C}=1.23$. The maximum deviation between a $K_{L M}$ value predicted by this LFER and an individually measured $K_{L M}$ was less than 0.1 log unit for all peptides studied here. The data and LFER are graphically shown in Figure 4.4, in which the number of $\mathrm{F}$ and $\mathrm{Y}$ units are plotted on the $\mathrm{x}$ and $\mathrm{y}$ axes, while the overall affinity is shown on the vertical $\mathrm{z}$ axis. The light blue-colored plane indicates the LFER found after the fit procedure while the data points represent the individual $K_{L M}$ values. The fitted $a$ values indicate that an additional $\mathrm{Y}$ unit gives an increase of affinity with a factor 7 , and an additional $\mathrm{F}$ with a factor 5 . In a multivalency context, and assuming that only the maximum-valency species is observed at the surface, $\mathrm{C}=$ $\log E M$, while $a_{Y}=\log \left(K_{i, Y} \cdot E M\right)$ and $a_{F}=\log \left(K_{i, F} \cdot E M\right)$. This would correspond to $K_{i, Y}=123 \mathrm{M}^{-1}, K_{i, F}=83 \mathrm{M}^{-1}$, and $E M=59 \mathrm{mM}$. These values compare favorably to literature values for the individual affinities $\left(K_{i, Y}=120 \mathrm{M}^{-1}, K_{i, F}=60 \mathrm{M}^{-1}\right)^{10}$ and the $E M$ (100 mM) found before for $\beta$-CD monolayers with adamantyl guests. ${ }^{8}$ Obviously, since their data were incorporated here and the fit quality was found to be high, the $\mathrm{Y}_{3}-\mathrm{Y}_{6}$ peptides studied separately in Chapter 3 gave very similar values for $K_{i, Y}, E M$, and the multivalent enhancement factor. 


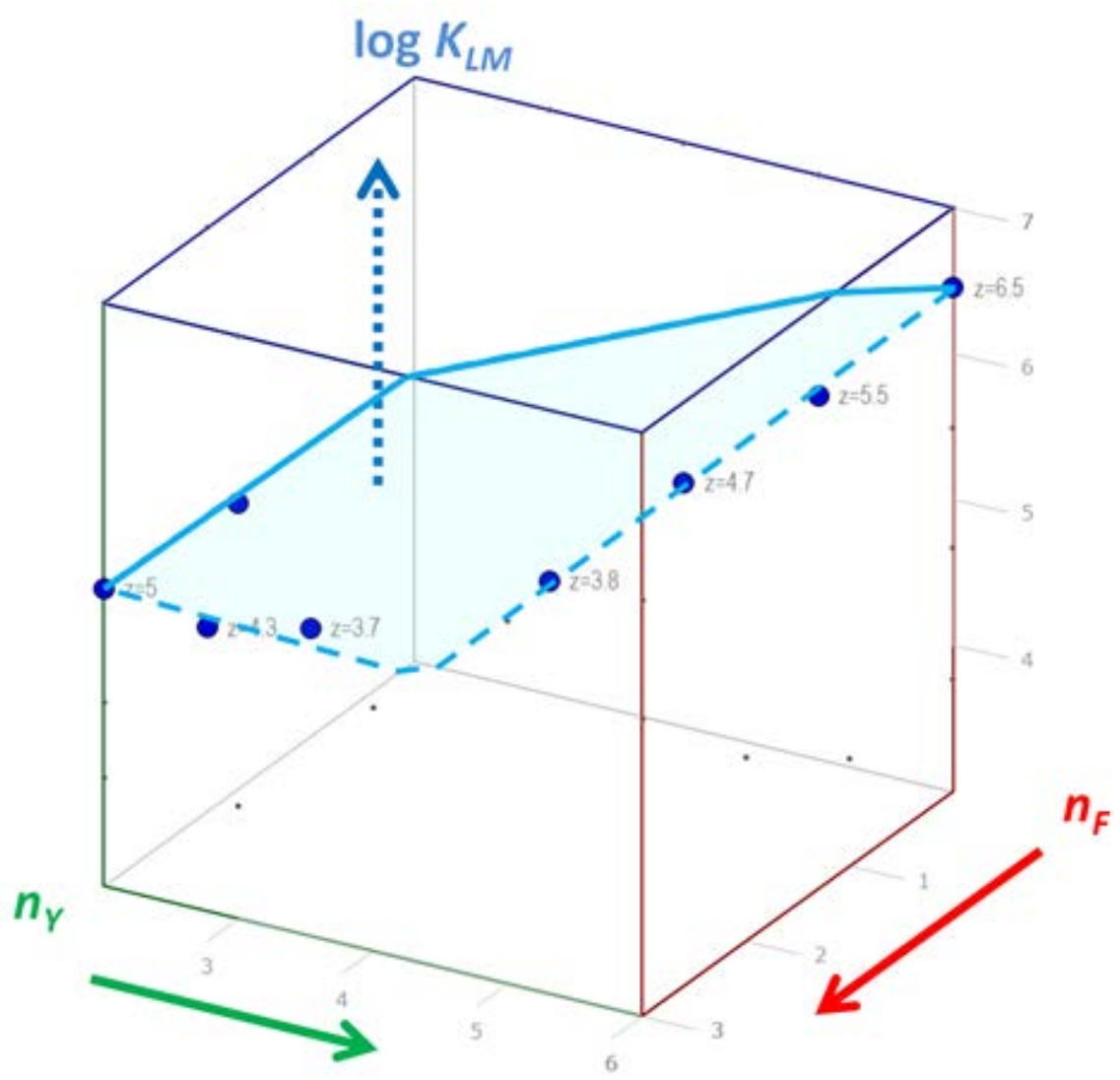

Figure 4.4 - 3D plot showing the influence of the numbers of $\mathrm{Y}$ and $\mathrm{F}$ sites in the linear peptides on the binding affinity $\left(\log K_{L M}\right)$. Log $K_{L M}$ values were fitted to a linear free energy relation (LFER). Solid lines of the blue-colored LFER plane run on outer exposed planes of the cube defined by $n_{F}=3, n_{Y}=6$, and $\log K_{L M}=7$, while the dashed lines run across inner cube planes $\left(n_{F}=0, n_{Y}=2\right.$, and $\left.\log K_{L M}=3\right)$.

Alternatively, as also discussed in detail in Chapter 3, we fitted the individual SPR graphs to the multivalency model (see fits in Figure 4.2). This model needs only independent parameters for $K_{i, Y}, K_{i, F}$, and $E M$, and allows to calculate the concentrations of every possible surface species (see also Chapter 6). As observed in Chapter 3, for only the Y-containing peptides, there is a strong compensation effect between $K_{i}$ and $E M$ when attempting to fit these parameters independently. Therefore, we fixed $K_{i, Y}=120 \mathrm{M}^{-1}, K_{i, F}=60 \mathrm{M}^{-1}$ to the values reported in literature, and optimized 
a single $E M$ value for all peptides together. The sum of errors for all calculated and measured SPR coupling angle data points of all titration curves together when using only this one variable, was only a factor 1.3 higher than when using four fit variables ( $K_{i, Y}, K_{i, F}$, and two $E M$ values for each of the peptide systems (Y-only or mixed Y/F peptides), as depicted in Figure 4.1d). The latter procedure provided unrealistically low $K_{i}$ values and too high $E M$ values. In the former case, when having a single $E M$ as the only fit parameter, an $E M$ of $43 \mathrm{mM}$ was found, in good agreement with the values found before and the one described in Chapter 3. Relaxing the model to allow two EM values for the two peptide systems separately, did not significantly improve the fit (factor 1.1 difference in the sum of errors). Whereas one could have expected different $E M$ values for each of the peptide series because they have different backbone sequences (SGGYGGS vs IFQEYPD) and different distances between the aromatic amino acids, the data shown here indicates that the structural difference is too little to have a significant effect on $E M$. This agrees well with the insensitivity of $K_{L M}$ found for Y-containing peptides with different inter-Y spacing, as described in Chapter 3.

The $K_{i}$ and $E M$ values found using the LFER and multivalency models compare well. The small discrepancies occur from: (i) intrinsically different fits of the individual SPR titration graphs between the Langmuir and multivalency models, because the multivalency model allows for changing of the species population with increasing coverage (see also Chapter 6) with a concomitant change of the average binding valency, and (ii) differences in the mathematical processes of linking all titration data together as outlined above. Using the multivalency model, multivalent enhancement factors $\left(K_{i} \cdot E M\right)$ of 5.2 and 2.6 were obtained for $\mathrm{Y}$ and $\mathrm{F}$, respectively. When incorporating all surface-bound species in calculating an overall affinity $K_{o v}$ (= $\left.\sum_{j=1}^{n}\left(\begin{array}{l}n \\ j\end{array}\right) K_{i}\left(K_{i} * E M\right)^{j-1}\right)$, see Chapter 3), these translate into an increase of $K_{o v}$ with a factor 6.2 and 4.0 for each additional Y or F unit, respectively, that is introduced into the peptide structure. As expected from the good fits of both models, also these values do not differ much from the values (7 and 5) mentioned above obtained from the LFER model. Regardless what model is chosen, the data indicate that the overall affinity is "programmable”, i.e., the affinity is systematically and predictably related to the number of $\mathrm{Y}$ and $\mathrm{F}$ units implemented in the peptide and their, independently measurable, intrinsic affinities. 


\subsubsection{Peptide dendrimer conjugates}

The results shown above indicate that the chosen bioactive peptides based on the IFQEYPD motif can be immobilized on the $\beta$-CD surface and that the binding affinity with the $\beta$-CD surface depends on the number and the type of the aromatic amino acids $\mathrm{Y}$ and $\mathrm{F}$ in a highly predictable manner. To couple peptides to dendrimers, we used carbonyldiimidazole (CDI) as a coupling agent as it is highly reactive towards hydroxyl, amino and carboxyl functional groups. ${ }^{11-13}$ For the synthesis of G0-(FY) $)_{4}$ we used a dendrimer with amine $\left(\mathrm{NH}_{2}\right)$ end groups and in the case of $\mathrm{G} 0.5-(\mathrm{FY})_{7}$ a dendrimer with carboxyl $(\mathrm{COOH})$ end groups while in the case of G2-(FY) $)_{15}$, G3-(FY) $)_{30}$, G6$(\mathrm{YFY})_{53}$ we used dendrimers with hydroxyl $(\mathrm{OH})$ end groups. The synthesis was carried out in two steps as shown in Scheme 4.1. The type of the bond formed after peptide coupling in step two in each dendrimer case is shown in Table 4.1.

\section{Step 1:}

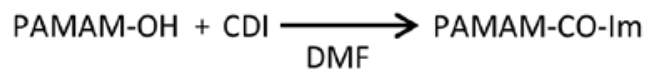

Step 2:

PAMAM-CO-Im + $\mathrm{NH}_{2}$-IFQEYPD-CONH DMF

PAMAM-CONH-IFQEYPD-CONH

Scheme 4.1 - Scheme showing two generalized functionalization steps involved in coupling of peptides to the end groups of PAMAM dendrimers, starting from activation of the dendrimer by CDI (step 1) followed by reaction with the peptide (step 2).

Activation of the dendrimers was confirmed using NMR spectroscopy. Excess of CDI was removed by a short washing step with water (which hydrolyzes the excess of free CDI), while activated PAMAM is known to be more stable towards water treatment. ${ }^{14}$ After the second step, complete disappearance of CDI was confirmed using NMR spectroscopy (details are given in the Materials and methods section). Of all strategies tried (shown in Materials and methods section) we found that the microwave-assisted 
procedure was shorter and yielded the highest extent of functionalization. In step two, a large excess of peptide was used under all the reaction conditions. After synthesis, the reaction mixture was first subjected to purification using preparative HPLC followed by size exclusion chromatography. From preparative HPLC it was observed that the functionalization was highest in the case of using microwave during the coupling step. The extents of functionalization were determined by UV/vis spectroscopy. Complete details of purification and characterization of the dendrimers is given in the Materials and methods section. Using the same strategy, various different types of dendrimer conjugates were prepared as shown in Table 4.1.

\begin{tabular}{|c|c|c|c|}
\hline Nomenclature & $\begin{array}{l}\text { Number of } \\
\text { end groups }\end{array}$ & $\begin{array}{c}\text { Number of } \\
\text { peptides } \\
\text { coupled }\end{array}$ & $\begin{array}{l}\text { Type of conjugation } \\
\text { bond formed }\end{array}$ \\
\hline $\mathrm{GO}-(\mathrm{FY})_{4}$ & 4 & 4 & \\
\hline G0-(YFY) $)_{2}$ & 4 & 2 & \\
\hline G0.5-(FY) $)_{7}$ & 8 & 7 & \\
\hline G2-(FY) 15 & 16 & 15 & \\
\hline G3-(FY) 30 & 32 & 30 & \\
\hline G3-(YFY) $)_{13}$ & 32 & 13 & \\
\hline G6-(YFY) $)_{53}$ & 256 & 53 & \\
\hline
\end{tabular}

Table 4.1 - Various types of peptide-dendrimer conjugates synthesized by coupling two different types of peptides (R), IFQEYPD (denoted by FY) and GYGIFQEYPD (denoted by YFY), to the terminal functional groups $\left(\mathrm{NH}_{2}, \mathrm{OH}\right.$, or $\left.\mathrm{COOH}\right)$ of various generations of PAMAM dendrimers 
(denoted by the number after the $G$ in the first column). The number of peptides coupled represents an experimental estimate of the extent of functionalization of the number of available end groups.

After the synthesis, the binding affinities of the dendrimers with the $\beta$-CD monolayer were determined by SPR, and $\log K_{L M}$ values were obtained by fitting the SPR titration data to a Langmuir binding model. It was observed that dendrimers containing YFY in their peptide sequence showed a higher binding affinity compared to dendrimers containing FY in the same sequence peptide, when coupled to same generation of PAMAM dendrimer. This can be attributed to average high Y content of the YFY dendrimers compared to FY containing dendrimers. From the $\log K_{L M}$ values of the dendrimers only, it was difficult to decipher the valency of interaction, i.e., the number of aromatic amino acids in each dendrimer interacting with the $\beta$-CD monolayer. Therefore we have used the data of the linear peptides to quantify the stoichiometry of the host-guest interactions in the globular dendrimers. In the case of dendrimers functionalized with the YF peptide, the ratio between $\mathrm{Y}$ and $\mathrm{F}$ is $1: 1$, so when implementing this in the LFER equation given above, this is reduced to:

$$
\log K_{L M, \text { calc }}=q\left(a_{Y}+a_{F}\right)+\mathrm{C}=1 / 2 n\left(a_{Y}+a_{F}\right)+\mathrm{C}
$$

where $q$ is the number of YF peptides interacting with the surface, and $n$ is the total valency of aromatic groups interacting with the surface. Similarly, for dendrimers functionalized with the YFY peptide, the equation is reduced to:

$$
\log K_{L M, \text { calc }}=q\left(2 a_{Y}+a_{F}\right)+\mathrm{C}=1 / 3 n\left(2 a_{Y}+a_{F}\right)+\mathrm{C}
$$

Therefore, when plotting $\log K_{L M}$ vs the valency $n$ (Figure 4.5), lines with slopes of $1 / 2\left(a_{Y}+a_{F}\right)=0.78$ and $1 / 3\left(2 a_{Y}+a_{F}\right)=0.80$ are obtained for the FY and YFY peptides. Figure 4.4 plots the measured $K_{L M}$ values of the dendrimers on the y axis and projects these values on the LFER lines given above. From the cross points, the corresponding $x$ axis values give the estimated valencies of the binding of the peptide-modified dendrimers, as reported in Table 4.2. For example, in the case of G0-(FY) ${ }_{4}$, the $\log K_{L M}$ value corresponds to a valency of close to 4 . Therefore, we presume that two FY peptide arms are involved in the interaction of the dendrimer with the $\beta$-CD surface. Because the dendrimer has a total of 4 peptide arms, 2 remain unbound and are exposed to the solution and available to interact with integrin receptors. Table 4.2 gives therefore the numbers of unused peptide arms as well as those numbers divided by the binding 
valency which is a measure for the density of unused peptide arms per unit surface area (here: per $\beta$-CD cavity). The density values of 4.5 and 5.5 for G3-(FY) 30 and G6$(\mathrm{YFY})_{53}$, respectively, are unrealistically high, probably indicating that steric hindrance will occur and will reduce the density. On the other hand, the weaker binding dendrimers probably leave a larger fraction of $\beta$-CD sites unused when binding to the surface, indicating that their density values might also be overestimated.

\begin{tabular}{|c|c|c|c|c|c|c|}
\hline $\begin{array}{c}\text { Type of } \\
\text { dendrimer }\end{array}$ & $\begin{array}{c}\text { Binding } \\
\text { affinity } \\
\left(\text { log } K_{L M}\right)\end{array}$ & $\begin{array}{c}\text { Valency of } \\
\text { interaction }\end{array}$ & $\begin{array}{c}\text { Number of } \\
\text { arms } \\
\text { interacting } \\
\text { with } \beta C D \\
\text { surface }\end{array}$ & $\begin{array}{c}\text { Unbound } \\
\text { peptide } \\
\text { arms }\end{array}$ & $\begin{array}{c}\text { Density of } \\
\text { unbound } \\
\text { peptide } \\
\text { arms per } \\
\beta C D \text { cavity }\end{array}$ & $\begin{array}{c}\text { Projected } \\
\text { density } \\
\text { of } \\
\text { unbound } \\
\text { peptide } \\
\text { arms per } \\
\beta C D \\
\text { cavity }\end{array}$ \\
\hline G0-(FY) $)_{4}$ & 4 & 4 & 2 & 2 & 0.5 & 0.7 \\
\hline G0-(YFY $)_{2}$ & 4.7 & 4 & 2 & 0 & 0 & 0 \\
\hline G0.5-(FY) & 5 & 5 & 3 & 4 & 0.8 & 1 \\
\hline G2-(FY) $)_{15}$ & 5.9 & 6 & 3 & 12 & 2 & 3 \\
\hline G3-(FY) $)_{30}$ & 6.1 & 6 & 3 & 27 & 4.5 & 3.4 \\
\hline G3-(YFY $)_{13}$ & 6.3 & 6 & 2 & 11 & 1.8 & 1.1 \\
\hline G6-(YFY $)_{53}$ & 8.1 & 9 & 3 & 50 & 5.5 & 2.2 \\
\hline
\end{tabular}

${ }^{1}$ Calculated assuming stretched conformations of dendrimers as shown in Figure 4.6

Table 4.2 - Table showing binding affinities $\left(K_{L M}\right)$, valencies of interaction calculated for each peptide dendrimer conjugate from binding affinity plots (Figure 4.5), and values, calculated from these valencies, for the number of interacting and unbound peptide arms and density of unbound peptides per $\beta$-CD-cavity. The density has alos been obtained from geometric considerations of dendrimers and $\beta$-CD surface (Figure 4.6). 

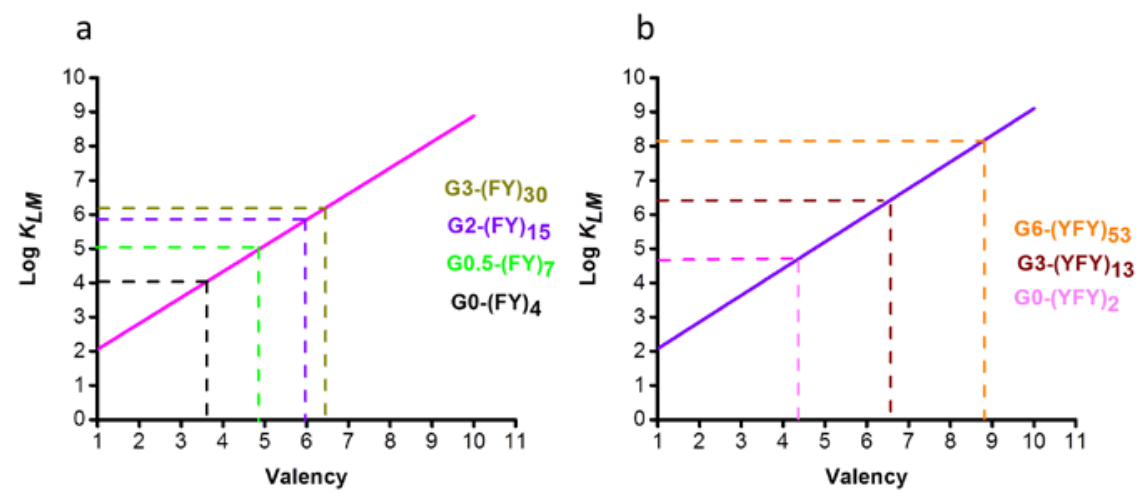

Figure 4.5 - Simulation plots of $\log K_{L M}$ vs valency (sum of Y and F units) for a) FY and b) YFY linear peptides using the LFER. Log $K_{L M}$ values obtained for peptide-dendrimer conjugates (shown by dashed lines at the y axes) projected onto the simulation lines to calculate the valency of interaction (with the $\beta$-CD surface) for each dendrimer.

To verify the experimental densities of unused peptide arms, we decided to make molecular models of the dendimers to estimate the projected numbers of $\beta$-CD cavities per dendrimer. As the dendrimers vary in size, the number of interaction sites and the resulting binding affinity with the $\beta$-CD surface also vary. These differences in the physical properties are known to influence the surface coverage, i.e., the total number of dendrimers present per surface area of the $\beta$-CD SAM. ${ }^{8}$ Cartoons depicting the sizes of the dendrimers and their projected surface areas on the $\beta$-CD SAM is shown in Figure 4.6. Sizes of the dendrimers and the distance of the tyrosine units from the dendrimer core was based on the assumption that they are present in a stretched conformation. 


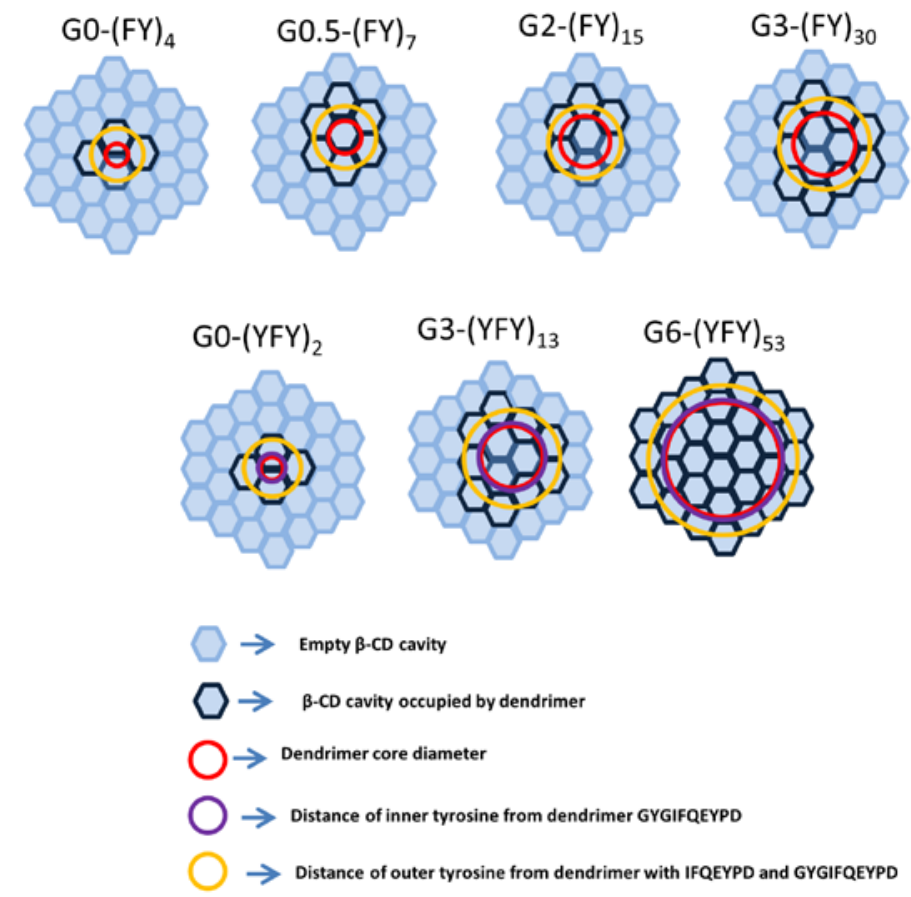

Figure 4.6 - Schematic representation of dendrimer coverages on the $\beta$-CD surface. All sizes of the dendrimers are scaled relative to the distance between two adjacent $\beta$-CD cavities (21 $\AA$ ). ${ }^{8}$

The pictorial representation in Figure 4.6 shows that the size of the G6-(YFY) $)_{53}$ dendrimer is relatively large when compared to the G0-(FY) $)_{4}$ and G0.5-(FY) dendrimers. Accordingly, more molecules of G0-(FY) $)_{4}$ or G0.5-(FY) $)_{7}$ can occupy the same surface area than that occupied by one molecule of G6-(YFY) $)_{53}$. The number of projected $\beta-C D$ cavities occupied by a dendrimer was translated subsequently into the density of unbound peptide units per $\beta$-CD cavity as shown in Table 4.3. These densities of unbound peptides were in good agreement with the values calculated from the binding valency for the lower generations of dendrimers. For the higher generations of dendrimers (G3-(FY) $)_{30}$, G3-(YFY) 13 and G6-(YFY) $)_{53}$ ), however, the modeled values were lower than obtained from the affinity data, as shown in Table 4.3, in particular for the G6 dendrimer. This indicates that the higher generation dendrimers can occupy a larger surface area compared to its binding valency, leaving some $\beta$-CD sites inaccessible by other dendrimers but unoccupied by guests. In contrast, in the case of 
the lower generations of dendrimers, the binding valencies of the guests are similar to the surface area occupied by the dendrimers. Yet, both strategies used to quantify the density of unbound peptides neglect the influence of surface coverage of the dendrimers on the $\beta$-CD SAM, whereas the coverage can have a strong influence on the binding valency (see Chapter 6).

To further quantify the coverage of dendrimers upon adsorption to the $\beta$-CD surface, quartz crystal microbalance (QCM) measurements were performed. By QCM the adsorbed mass on the surface is quantified by measuring a vibrational frequency change. From the observed frequency dampening (Figure 4.11), the mass adsorbed of each dendrimer was calculated, using the Sauerbrey equation. Table 4.3 shows the adsorbed masses for the three dendrimers (G6-(YFY) $)_{53}, \mathrm{G3}-(\mathrm{YFY})_{13}$, and G3-(FY) 30 ) studied here. The adsorbed mass can also be obtained from the SPR data reported above, in particular from the limiting SPR angle shifts. This requires conversion of the angle shift into adsorbed mass, calculated from the SPR angle shift where 1.00 mdeg $=1.05 \mathrm{ng} / \mathrm{cm}^{2}$ of analyte mass adsorbed. ${ }^{16}$ The adsorbed masses per surface area as calculated from the SPR data (Figure 4.7) shows a good agreement with the values obtained by QCM. The adsorbed mass did not increase with the size of the dendrimers, as in the case of G6-(YFY $)_{53}$ a significantly lower adsorbed mass was observed. It suggests that $\beta$-CD SAMs are not fully packed when adsorbing G6$(\mathrm{YFY})_{53}$. Possibly, the binding data obtained with SPR and QCM experience more mass transport limitation due to the very high molecular weight of this dendrimer. The adsorbed masses, using the values obtained from SPR, were converted into coverage of dendrimer molecules per surface area. Next, taking into account the numbers of peptide arms on the dendrimers that are not interacting with the $\beta$-CD surface, we calculated the exposed ligand density per surface area for each dendrimer (Table 4.3). 


\begin{tabular}{|c|c|c|c|c|}
\hline $\begin{array}{c}\text { Type of } \\
\text { dendrimer }\end{array}$ & $\begin{array}{c}\text { Mass } \\
\text { adsorbed } \\
\left(\mathrm{ng} / \mathrm{cm}^{2}\right) \\
\text { from SPR }\end{array}$ & 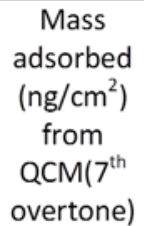 & $\begin{array}{c}\text { Number of } \\
\text { unbound } \\
\text { peptides per } \\
\mathrm{cm}^{2} \\
\left(10^{11}\right)\end{array}$ & $\begin{array}{c}\text { Density of } \\
\text { unbound } \\
\text { peptides } \\
\text { per } \beta-C D \\
\text { cavity }\end{array}$ \\
\hline $\mathrm{GO}-(\mathrm{FY})_{4}$ & 1.4 & - & 4.2 & 0.011 \\
\hline $\mathrm{G} 0.5-(\mathrm{FY})_{7}$ & 3.2 & - & 13.3 & 0.037 \\
\hline $\mathrm{G} 2-(\mathrm{FY})_{15}$ & 5.6 & - & 23.2 & 0.064 \\
\hline G3-(FY) $)_{30}$ & 20.1 & 20.3 & 96.9 & 0.271 \\
\hline G3-(YFY) $)_{13}$ & 5.3 & 3.3 & 14.1 & 0.039 \\
\hline G6- $(Y F Y)_{53}$ & 2.8 & 2.6 & 6.7 & 0.018 \\
\hline
\end{tabular}

Table 4.3 - Table showing the mass adsorbed per surface area calculated independently from SPR and QCM measurements. The mass adsorbed from SPR is converted to exposed ligand density by multiplying the number of molecules per surface area (from adsorbed mass) with the unbound peptide density from Table 4.2.

\subsection{Conclusions}

In conclusion, the multivalency of peptide-conjugated dendrimers was programmed to gain control over the exposed peptide density on the $\beta$-CD surface. In these peptidedendrimer conjugates, individual contributions from tyrosine and phenylalanine on the binding affinity was calculated using LFER modeling of the SPR data. This model was further extrapolated to generate simulation lines for two types of bioactive peptides, IFQEYPD and GYGIFQEYPD. These two types of peptides were coupled to the end groups of various generations of PAMAM dendrimers. It has been observed that, due to the globular nature of the PAMAM dendrimers, not all peptide moieties were able to interact with the $\beta$-CD surface. The valency of interaction of each dendrimer with the $\beta$ CD surface was calculated from the affinity data and from geometrical esimations. The $\log K_{L M}$ values of the dendrimers obtained from fitting of the SPR data, was projected onto LFER simulation lines to extract the valencies of interaction. This valency calculation gave an idea of how many of peptides are not interacting with the $\beta$-CD surface. From SPR and QCM measurements the mass of each dendrimer adsorbed on $\beta$ CD surface was estimated. This mass adsorbed per surface area was then multiplied with exposed ligand density per dendrimer to calculate exposed ligand density per 
surface area for each dendrimer. The methodology as a whole has allowed us to determine the number of peptides binding to the surface as well as the exposed peptide densities, and affinity and molecular structure contributions have been elucidated. The exposed peptide density will be further exploited in Chapter 5 to elicit integrin clustering mediated VEGF signaling in cellular and in vivo conditions.

\subsection{Acknowledgements}

Prof. Jurriaan Huskens and Prof. Pascal Jonkheijm are highly acknowledged for their help with modeling of SPR and QCM data.

\subsection{Materials and Methods}

General Chemicals were obtained from commercial sources and used as received. Fmoc-Rink Amide MBHA resin (0.52 mmol/g), Fmoc-protected amino acids and related coupling agents for peptide synthesis were purchased from Multisyntech GmbH Chemicals. Solvents employed in the purification of peptides were purchased from Sigma-Aldrich. Dimethylformamide (DMF) was distilled over magnesium sulfate and stored over $4 \AA$ molecular sieves and DOWEX 50WX8-400 strongly acidic resin under $\mathrm{N}_{2}$. Water used was MQ grade. All other reagents were purchased from Aldrich (Milwaukee, WI), Sigma (St. Louis, MO), Advanced Chemtech (Louisville, KY), Biosolve (Netherlands), Multisyntech (Netherlands) and used without further purification.

Equipment All reactions were performed in a special microwave reactor provided by CEM Corporation (U.S.A.) used with a single channel automated microwave synthesizer. ${ }^{1} \mathrm{H}$ and ${ }^{13} \mathrm{C}$ NMR spectra were recorded on a Bruker AC-600 (600 MHz) spectrometer. Size exclusion chromatography was performed using Superdex-75 column with MWCO 3000-70000 (GE Healthcare). A JMS-T100LC mass spectrometer was used to determine the mass of the peptides. High-performance liquid chromatography (HPLC) experiments were performed with equipment from Waters. Surface plasmon resonance (SPR) experiments were conducted using $50 \mathrm{~nm}$ SPR gold substrates from Ssens BV using equipment from Resonant-probes GmbH. The angle of 
minimum reflection was tracked during the experiments. The gold substrate with the monolayer was optically matched to the prism using an index matching oil. A flow cell was placed on the monolayer and was filled by intended solutions with a syringe pump. The solutions of guest molecules were prepared with phosphate buffer. A $5 \mathrm{mM}$ solution of cyclodextrin in phosphate buffer and the phosphate buffer itself were used to wash the substrates and to establish the baseline in the SPR experiments.

\section{Synthesis}

Peptides were synthesized with solid phase peptide synthesis with Fmoc-Rink Amide MBHA resin, following a standard Fmoc protocol. Purification of the peptide was done using reversed phase HPLC. The quality of peptide was investigated by analytical HPLC and ESI mass spectra. Preparative column (Waters 2535 quaternary gradient module with XBridge ${ }^{\mathrm{TM}}$ prep C18 $5 \mu \mathrm{m} \mathrm{OPD}^{\mathrm{TM}} 19 \times 250 \mathrm{~mm}$ preparative column) with eluents $0.1 \%$ aqueous TFA and $0.1 \%$ TFA in acetonitrile were used to purify the crude peptides. The extent of purification was checked using an analytical HPLC (Waters 2535 quaternary gradient module XBridge C18 $5 \mu \mathrm{m}$ column with $4.6 \times 250 \mathrm{~mm}$ dimensions). Same eluents as that of preparative HPLC were used for analytical HPLC. HPLC retention times were observed following analytical HPLC with a solvent gradient of $0-90 \%$ acetonitrile over a 90 min period. The mass of the peak corresponding to the purified peptide was determined using LC-MS (Waters 2535 module coupled to micromass LCT)).

\section{Ac(SGGYGIFQEYPD)}

Peptide Ac(SGGYGIFQEYPD) was synthesized and purified, with a yield of $6.32 \mathrm{mg}$, 3.7\%. ESI mass spectra of peptide Ac(SGGYGIFQEYPD) confirmed the successful synthesis of the peptide. Chemical Formula: $\mathrm{C}_{63} \mathrm{H}_{83} \mathrm{~N}_{13} \mathrm{O}_{22}$, Mw: $1374.47(\mathrm{M}+\mathrm{H})$; found: $1373.32(\mathrm{M}+\mathrm{H})$. The analytical HPLC showed a single peak, indicating a product of high purity.

\section{Ac(IFQEYPDIFQEYPD)}

Peptide Ac(IFQEYPDIFQEYPD) was synthesized and purified, with a yield of 8.43 mg, 4.9\%. ESI mass spectra of peptide Ac(IFQEYPDIFQEYPD) confirmed the 
successful synthesis of the peptide. Chemical Formula: $\mathrm{C}_{88} \mathrm{H}_{116} \mathrm{~N}_{16} \mathrm{O}_{28}$, Mw: 1844.81(M+H); found: 921.35(M+H $)^{2+}$. The analytical HPLC showed a single peak, indicating product of high purity.

\section{Ac(IFQEYPDIFQEYPDIFQ)}

Peptide Ac(IFQEYPDIFQEYPDIFQ) was synthesized and purified, with a yield of 5.72 mg, 4.2\%. ESI mass spectra of peptide Ac(IFQEYPDIFQEYPDIFQ) confirmed the successful synthesis of the peptide. Chemical Formula: $\mathrm{C}_{108} \mathrm{H}_{114} \mathrm{~N}_{20} \mathrm{O}_{38}$, Mw: 2233.03(M+H); found: $1142.23(\mathrm{M}+2 \mathrm{Na})^{2+}$. The analytical HPLC showed a single peak, indicating product of high purity.

\section{Ac(IFQEYPDIFQEYPDIFQEYPD)}

Peptide Ac(IFQEYPDIFQEYPDIFQEYPD) was synthesized and purified, with a yield of $3.69 \mathrm{mg}, 1.2 \%$. ESI mass spectra of peptide Ac(IFQEYPDIFQEYPDIFQEYPD) confirmed the successful synthesis of the peptide. Chemical Formula: $\mathrm{C}_{131} \mathrm{H}_{172} \mathrm{~N}_{24} \mathrm{O}_{41}$, Mw: $2737.21(\mathrm{M}+\mathrm{H})$; found: $1369.08(\mathrm{M}+\mathrm{H})^{2+}$. The analytical HPLC showed a single peak, indicating product of high purity.

\section{Ac(IYQEYPDIYQEYPD)}

peptide Ac(IYQEYPDIYQEYPD) was synthesized and purified, with a yield of 6.72 mg, 3.8\%. ESI mass spectra of peptide Ac(IYQEYPDIYQEYPD) confirmed the successful synthesis of the peptide. Chemical Formula: $\mathrm{C}_{88} \mathrm{H}_{116} \mathrm{~N}_{16} \mathrm{O}_{30}$, Mw: 1876.80 $(\mathrm{M}+\mathrm{H})$; found: $939.44(\mathrm{M}+\mathrm{H})^{2+}$. The analytical HPLC showed a single peak, indicating product of high purity. 
AC SGGYGIFQEYPD

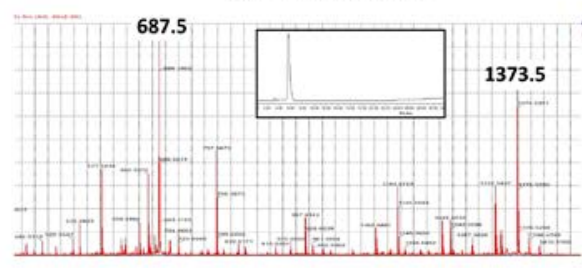

ACIFQEYPDIFQEYPDIFQ

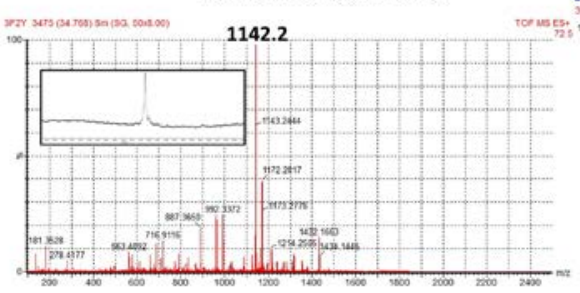

ACIFQEYPDIFQEYPD

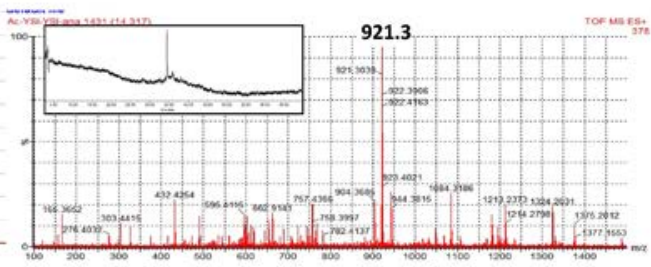

Ac IFQEYPDIFQEYPDIFQEYPD

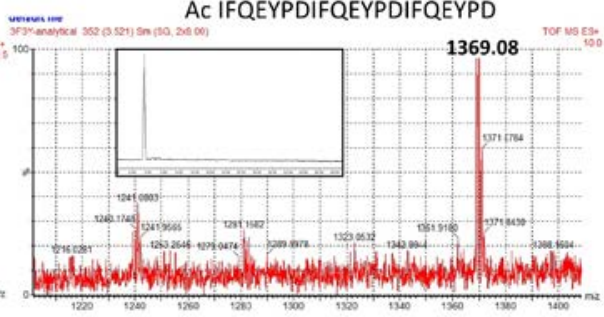

Figure 4.7- Representative mass and HPLC traces of purified peptides.

\section{Dendrimer synthesis and characterization}

Dendrimer synthesis was carried out following different reaction conditions to optimize the extent of functionalization of dendrimer with peptide as shown in Table 4.5. Microwave single coupling strategy was found to yield maximum extent of functionalization in G2-(FY) ${ }_{15}$ dendrimer (determined using HPLC and UV technique) and was used for synthesis of all the dendrimers.

\begin{tabular}{|c|c|c|c|c|}
\hline Sr. No. & Strategy & Temperature & Time (1" step) & Time $\left(2^{\text {nd }}\right.$ step) \\
\hline 1 & At room temperature & RT & $4 \mathrm{~h}$ & $24 \mathrm{~h}$ \\
\hline 2 & Conventional heating & $55^{\circ} \mathrm{C}$ & $4 \mathrm{~h}$ & $12 \mathrm{~h}$ \\
\hline 3 & Conventional heating & $55^{\circ} \mathrm{C}$ & $4 \mathrm{~h}$ & $24 \mathrm{~h}$ \\
\hline 4 & $\begin{array}{l}\text { Microwave single } \\
\text { coupling }\end{array}$ & $70^{\circ} \mathrm{C}$ & $10 \mathrm{~min}$ & $20 \mathrm{~min}$ \\
\hline 5 & $\begin{array}{l}\text { Microwave double } \\
\text { coupling }\end{array}$ & $70^{\circ} \mathrm{C}$ & $10 \mathrm{~min}$ & $\begin{array}{c}40 \mathrm{~min}(20 \mathrm{~min} \\
\text { each cycle) }\end{array}$ \\
\hline
\end{tabular}

Table 4.5 - Various strategies used to optimize the reaction conditions for step 1 and step 2 in coupling protocol. 
After synthesis its purification was done with preparative HPLC. A difference in a retention time of peptide-dendrimer conjugates under different reaction conditions was observed (Figure 4.8). UV calibration plots indicated that these differences in retention times were due to different extent of functionalization of dendrimer core with that of peptides.

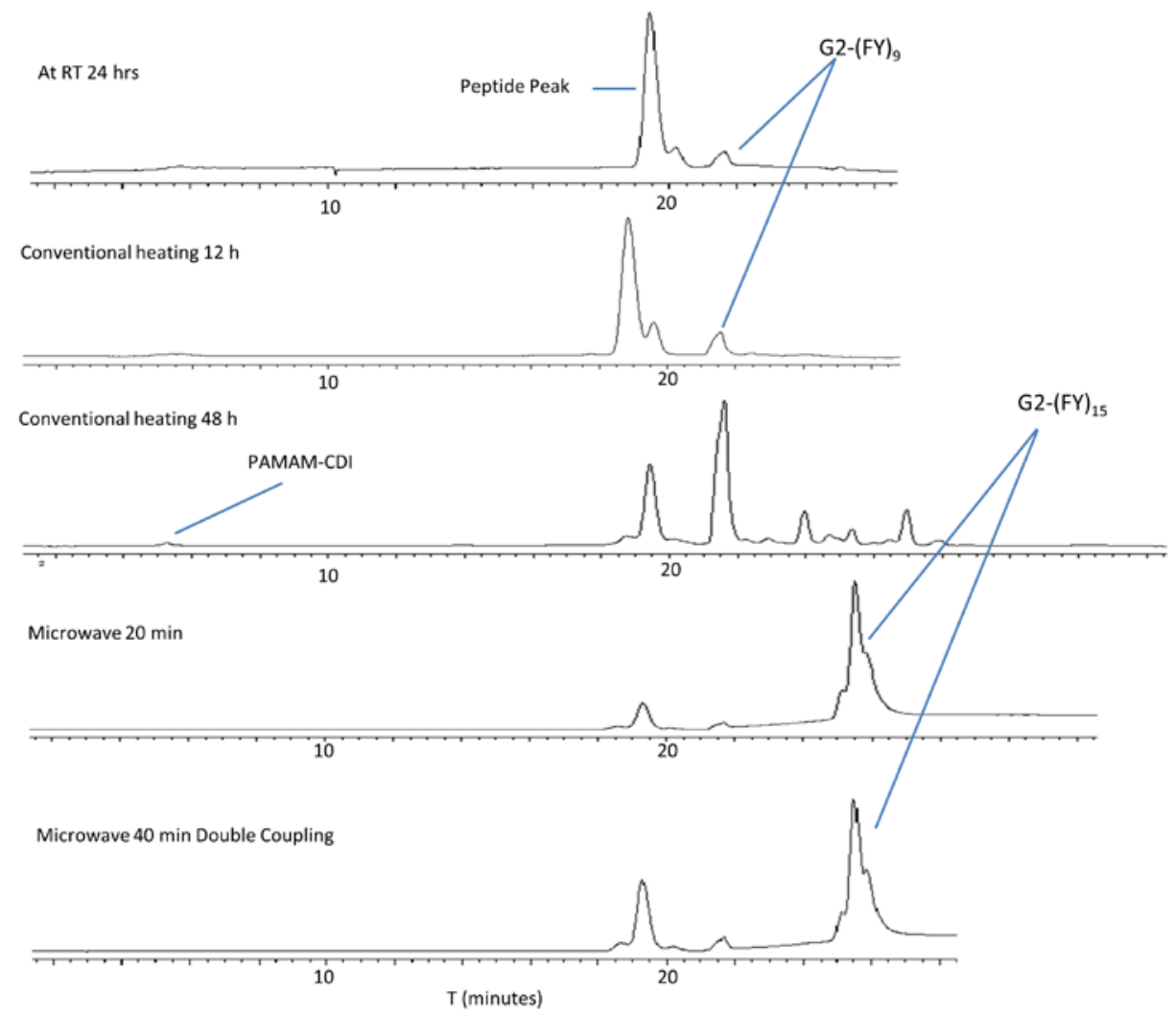

Figure 4.8 - Preparative HPLC chromatograms of peptide dendrimer conjugate (IFQEYPD coupling to end terminal of PAMAM G2.0) under different reaction conditions monitored at 280 nm.

The extent of functionalization of the dendrimers was quantified using UV/vis absorption spectroscopy following a reported procedure. ${ }^{17}$ A series of peptides (50, 100, 250, 500, 750, $1000 \mu \mathrm{g} \mathrm{mL}{ }^{-1}$ ) was prepared in methanol and their absorbance's were measured. We chose the UV absorbance at $280 \mathrm{~nm}$ as an indicator of the number of tyrosine's in a peptide. A standard curve of peptide concentration vs absorbance (at 280 
$\mathrm{nm}$ ) was plotted. The absorbance of unconjugated dendrimer was subtracted from the absorbance of the peptide-dendrimer conjugate products. Subtracted peptide-dendrimer conjugate absorbance's were compared with the calibration curve to estimate the amount of peptides that were conjugated to the dendrimers.

In liaison with UV/vis spectroscopy, NMR spectroscopy was used to further ensure the complete removal of CDI after peptide conjugation (Figure 4.9). The disappearance of CDI was confirmed (peaks 1, 2 and 3) with simultaneous evolution of tyrosine peaks after peptide conjugation to dendrimer in step 2. Peaks for PAMAM dendrimer were assigned according to literature. ${ }^{18}$ Further integration of protons was difficult due to large number of protons from dendrimer as well as peptides. Further attempts to characterize the dendrimer conjugates using matrix-assisted laser desorption ionization time-of-flight mass spectrometry (MALDI-TOF MS) failed as it is reported in the literature the choice of matrix is critical to get general molecular weight distribution in case of dendrimers. ${ }^{19}$

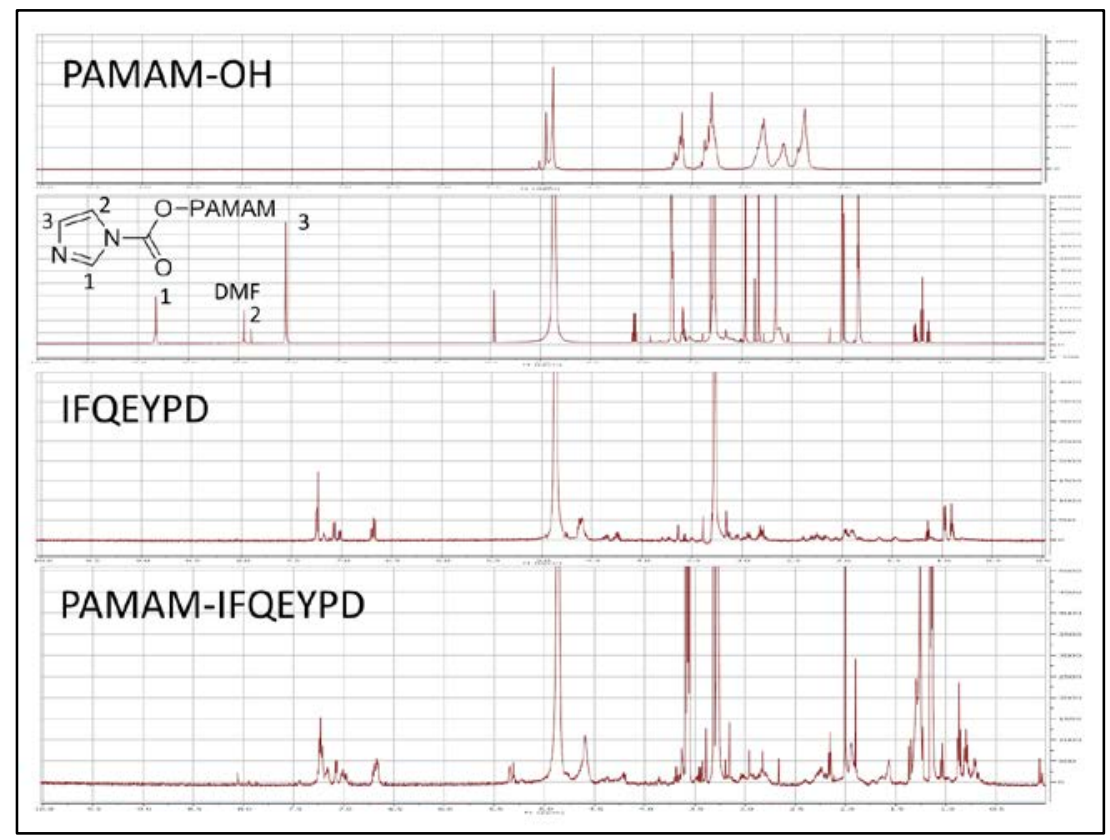

Figure 4.9- ${ }^{1} \mathrm{H}$ NMR spectra of various dendrimer derivatives. a) PAMAM-OH b) PAMAM coupled to CDI c) Starting peptide IFQEYPD; d) PAMAM-Peptide conjugate. 

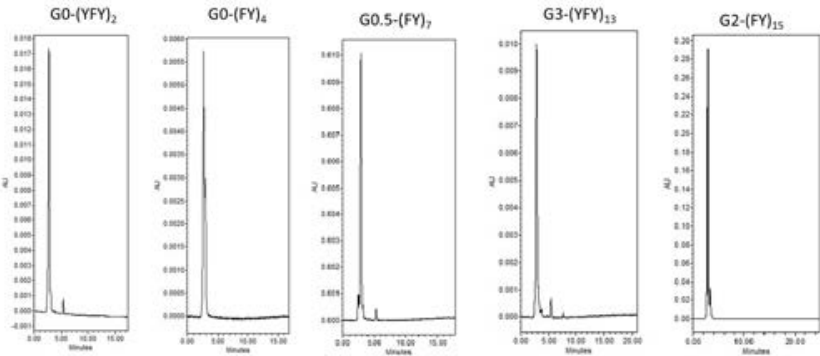

G0.5-(FY),

1044.40

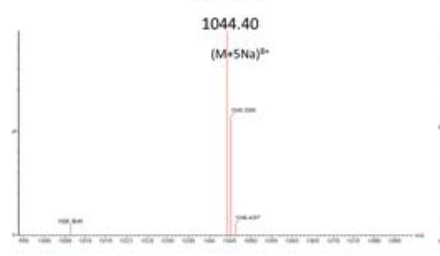

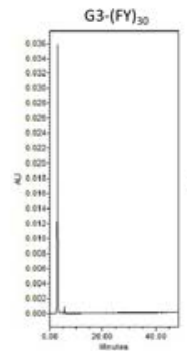

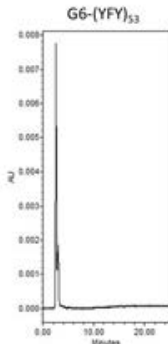

$\mathrm{G} 3 \cdot(\mathrm{FY})_{30}$

1689.20

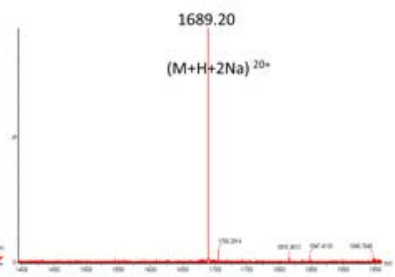

Figure 4.10- Representative HPLC and ESI-MS traces of purified dendrimer conjugates.

\section{QCM Measurements}

Measurements were performed on Q-Sense E4 system with QE 401 electronic unit, QCP 401 chamber platform and QFM 401 flow module. Gold coated disc (QSX 301) used for QCM measurement has the same dimensions as SPR disc and $\beta$-CD monolayer was fabricated on QCM disc using same protocol as that for SPR disc.

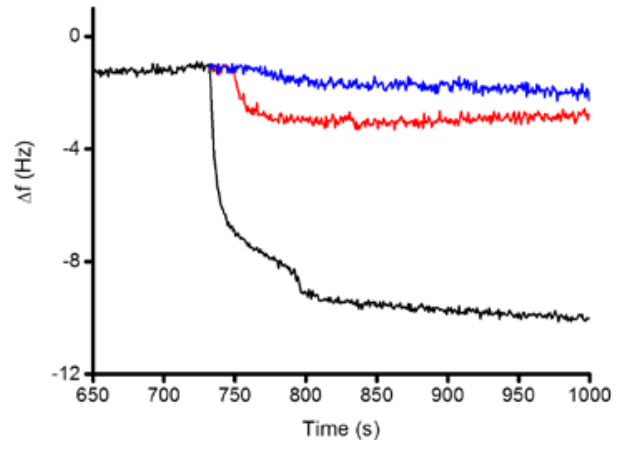

Figure 4.11 - QCM time traces showing the adsorption of dendrimers G6-(YFY) 53 (blue), G3$(\mathrm{YFY})_{13}$ (red), G3-(FY) $)_{30}$ (black) on the $\beta$-CD SAM at a concentration of each dendrimer for which saturation of the signal was observed in SPR measurements. 


\section{SPR titration of peptide/dendrimers}

SPR titration of the bioactive peptide/dendrimers was done similarly as described in

Chapter 3. Briefly, different concentrations of each sample was flowed over the $\beta$-CD surface and its angle change was measured till the concentration where no further enhancement in angle change was observed (surface saturating concentration). Due to high binding affinity of dendrimer with $\beta$-CD surface in most of the cases new SPR disc was used for each measurement.

\subsection{References}

1. Brinkmann, J., Cavatorta, E., Sankaran S.,Schmidt, B., van Weerd, J. \& Jonkheijm, P. About Supramolecular systems for dynamically probing cells. Chemical Society Reviews 43, 4449-4469 (2014).

2. Neirynck, P., Schimer, J., Jonkheijm, P. \& Brunsveld, L. Carborane-[small beta]-cyclodextrin complexes as a supramolecular connector for bioactive surfaces. Journal of Materials Chemistry B 3, 539-545 (2015).

3. Cabanas-Danés, J. \& Jonkheijm, P. A supramolecular host-guest carrier system for growth factors employing VHH fragments. Journal of the American Chemical Society 136, 12675-12681 (2014).

4. Ludden, M.J.W., Reinhoudt, D.N. \& Huskens, J. Molecular printboards: versatile platforms for the creation and positioning of supramolecular assemblies and materials. Chemical Society Reviews 35, 1122-1134 (2006).

5. Mulder, A. \& Huskens, J. Molecular printboards on silicon oxide: lithographic patterning of cyclodextrin monolayers with multivalent, fluorescent guest molecules. Small 1, 242-253 (2005).

6. Boekhoven, J., Rubert Pérez, C.M., Sur, S., Worthy, A. \& Stupp, S.I. Dynamic display of bioactivity through host-guest chemistry. Angewandte Chemie International Edition 52, 12077-12080 (2013).

7. Huskens, J., Deij, M.A. \& Reinhoudt, D.N. Attachment of molecules at a molecular printboard by multiple host-guest interactions. Angewandte Chemie International Edition 41, 4467-4471 (2002). 
8. Huskens, J. A Model for describing the thermodynamics of multivalent host-guest interactions at interfaces. Journal of the American Chemical Society 126, 6784-6797 (2004).

9. Oommen, S., Gupta, S.K. \& Vlahakis, N.E. Vascular endothelial growth factor A (VEGF-A) induces endothelial and cancer cell migration through direct binding to integrin $\alpha 9 \beta 1$ : identification of a specific $\alpha 9 \beta 1$ binding site. Journal of Biological Chemistry 286, 1083-1092 (2011).

10. Rekharsky, M. \& Inoue, Y. Chiral recognition thermodynamics of $\beta$ cyclodextrin: the thermodynamic origin of enantioselectivity and the enthalpy-entropy compensation effect. Journal of the American Chemical Society 122, 4418-4435 (2000).

11. Pathak, S., Singh, A.K., McElhanon, J.R. \& Dentinger, P.M. Dendrimeractivated surfaces for high density and high activity protein chip applications. Multivalency 20, 6075-6079 (2004).

12. Yui, N. Inhibitory effect of supramolecular polyrotaxane-dipeptide conjugates on digested peptide uptake via intestinal human peptide transporter. Bioconjugate Chemistry 13, 582-587 (2002).

13. Zhang, X. Formation of urea dipeptides from carbonyldiimidazole: application toward the protease inhibitors GE 20372 and MAPI. The Journal of Organic Chemistry 62, 6420-6423 (1997).

14. Podlech, J. in Houben-Weyl: Methods of organic chemistry additional and supplementary volumes to the $4^{\text {th }}$ edition. , Vol. E 22A 534-5372002).

15. Marx, K.A. Quartz crystal microbalance: a useful tool for studying thin polymer films and complex biomolecular systems at the solution-surface interface. Biomacromolecules 4, 1099-1120 (2003).

16. Wangkam, T. Adsorption of bovine serum albumin (bsa) on polystyrene (ps) and its acid copolymer. Current Applied Physics 12, 44-52 (2012).

17. Myung, J.H., Gajjar, K.A., Saric, J., Eddington, D.T. \& Hong, S. Dendrimermediated multivalent binding for the enhanced capture of tumor cells. Angewandte Chemie International Edition 50, 11769-11772 (2011).

18. Gomez, M.V., Guerra, J., Velders, A.H. \& Crooks, R.M. NMR characterization of fourth-generation PAMAM dendrimers in the presence and 
absence of palladium dendrimer-encapsulated nanoparticles. Journal of the American Chemical Society 131, 341-350 (2008).

19. Restani, R.B. Biocompatible polyurea dendrimers with ph-dependent fluorescence. Angewandte Chemie International Edition 51, 5162-5165 (2012). 


\title{
Chapter 5
}

\section{Peptide-functionalized dendrimers on $\beta$-CD substrates as platforms to trigger VEGF signaling}

\begin{abstract}
In this chapter, peptide conjugated dendrimers immobilized onto $\beta$-CD printboards have been used to trigger the vascular endothelial growth factor (VEGF) activity. Different dendrimers with varying degrees of bioactive peptides have been prepared. The bioactive peptide IFQEYPD has been selected for its reported affinity towards $\alpha 9 \beta 1$ integrin and for its ability to form host-guest interactions with $\beta$-CD through its aromatic amino acids F and Y. Due to steric constraints, the immobilized dendrimers have remaining unbound peptides that were able to interact with the extracellular domain of integrin proteins. Variations in the density of unbound peptides on the dendrimer surface have been used to control the $\alpha 9 \beta 1$ integrin receptor clustering on the surface of the human microvascular endothelial cells (HMVECs). The $\alpha 9 \beta 1$ integrin clustering-mediated VEGF-A activation has been studied in vitro and in vivo. Moreover, the supramolecular system was fabricated on a biologically relevant polymer substrate for use in advanced biomedical applications.
\end{abstract}




\subsection{Introduction}

Cell surface receptors play an important role in coordinating interactions with the extracellular environment and this leads to various intracellular events. ${ }^{1}$ Most of these cell surface receptors do not act as an individual entity but in dimeric or oligomeric complexes. The literature suggests that depending on the number and the type of the clustered receptors, cells show a different extent of signaling response. ${ }^{2,3}$ Most of the growth factors present in the extracellular environment also exhibit their activity via these extracellular matrix receptors. ${ }^{4}$ The vascular endothelial growth factor (VEGF) is one of the most important growth factors because of its involvement in the vasculogenesis and angiogenesis pathways that are crucial for embryogenesis and new blood vessel development. ${ }^{5}$ VEGF acts via various different types of receptors that involve proteoglycans such as heparin-sulphate and the integrin-receptor subfamily. The primary receptor by which VEGF prefers to interact with the cells are the VEGFR-1, VEGFR-2, and VEGFR-3 receptors. ${ }^{6,7}$ As VEGF acts via different types of the receptor system, it is difficult to assign the dependence of VEGF activity to a particular receptor system under in vivo conditions.

Various strategies have been reported to gain control over growth factor activity, which involves covalent coupling of the growth factor to a solid surface or incorporating it in a polymeric matrix. ${ }^{8,9}$ However, these strategies suffer from some drawbacks, such as the high amount of growth factor required and the lack of spatiotemporal control over growth factor activity. Recently a strategy was used to circumvent these two important issues. $^{10}$ In this strategy peptide-functionalized surfaces were used to trigger a “clustering” of the cell surface receptors (T $\beta R I$ and T $\beta R I I)$ used by the growth factor TGF- $\beta$. This receptor clustering then acted as a trigger for the recruitment of TGF- $\beta$ from a surrounding medium to these receptor cluster sites. A similar strategy used heparin from serum as a trigger for the recruitment of the FGF-2 growth factor on the peptide-functionalized surface. ${ }^{11}$ Both strategies involved covalent modification of a surface with the active peptide. This covalent modification, however, spatially restricts the ligands, which might affect the receptor clustering ability. Alternatively, various non-covalent supramolecular strategies have been used to promote ligand-mediated receptor clustering. ${ }^{12-15}$ 
Inspired by the plausible flexibility of ligands on supramolecular platforms, we present herein a strategy to nucleate the VEGF signaling complex on a supramolecular selfassembling platform. The VEGFR2- $\beta 1$ integrin cross-talk is a long suspected trigger for VEGF activation.,8,16 Recently, a peptide sequence (IFQEYPD) has been reported which is specific for the $\alpha 9 \beta 1$ integrin receptors present on the HMVEC cell surface. This sequence neither activates nor requires the VEGFR-2 receptor which is the primary receptor for VEGF activation. ${ }^{7}$ We envisioned that the clustering of the integrin receptors could be induced by the previously developed (Chapter 4) dendrimer platforms, causing the clustering to act as a trigger for the recruitment of VEGF at the receptor cluster sites.

\subsection{Results and discussion}

\subsubsection{Preorganization of VEGF: A bio-signaling complex on a $\beta$-CD SAM}

Figure 5.1 shows our strategy used to trigger VEGF-A activity on supramolecular platforms. Firstly, different types of dendrimers immobilized on surfaces were investigated for their integrin clustering ability using the isolated $(\alpha 9 \beta 1)$ integrin receptors as well as whole cells. Next the translation of the integrin clustering ability in the stimulation of VEGF was investigated using in vitro and in vivo experiments. 
a
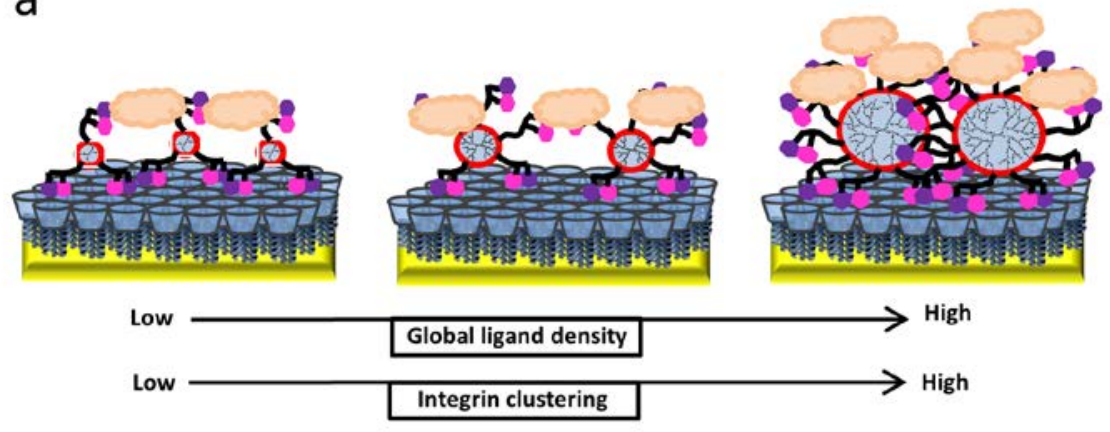

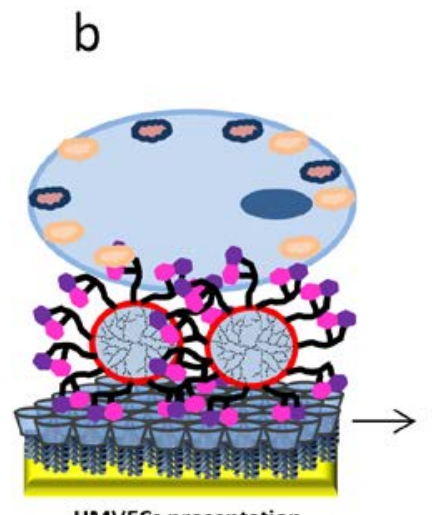

HMVECs presentation

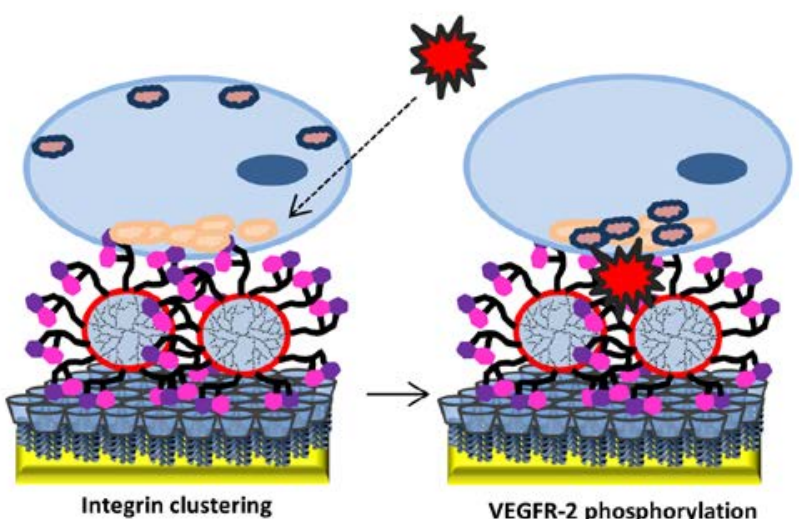

Integrin clustering

VEGFR-2 phosphorylation

VEGFR-2

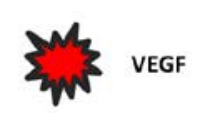

Figure 5.1 - Concept figure showing (a) the differences in the exposed ligand density to control an extent of integrin clustering, and (b) the stepwise assembly of each component to promote the final recruitment of the VEGF-A growth factor at the receptor cluster site.

\subsubsection{Effect of variation in exposed peptide density on integrin clustering}

In Chapter 4, the variation in the number of dendrimer-peptide valency was quantified using various techniques after their immobilization on the surface. For all dendrimers, the increase in the size and the extent of functionalization of the dendrimer led to the enhancement of the number of exposed (unbound) peptide per dendrimer. In the case of G6-(YFY) $)_{53}$, the surface coverage of the dendrimer was found to be low (see Chapter 4). Hence, even though the number of peptides per dendrimer was the highest in this 
case, the exposed peptide density remained low due to a low surface coverage. Impact of this variation of the exposed peptide density across the series of dendrimers on their ability to induce integrin clustering and VEGF triggering is described in the subsequent sections of this chapter.

In order to assess the integrin clustering ability of our platforms, an SPR experiment was designed where this ability was studied without the influence of any other cellular or extracellular factors. Each dendrimer was flowed over the $\beta$-CD monolayer at a surface saturating concentration (as found previously from SPR experiments, Chapter 4). After stabilization of the signal for $10 \mathrm{~min}$, a freshly prepared $2 \mu \mathrm{g} / \mathrm{ml}$ solution of commercially available $\alpha 9 \beta 1$ integrin was flowed through the SPR cell for $10 \mathrm{~min}$ followed by 10 min of stabilization and a PBS wash step. Each step of the dendrimer immobilization and the integrin adsorption was recorded (Figure 5.2a). A difference in the angle change between the dendrimer assembly and the integrin adsorption was recorded as a function of time. A control experiment consisted of flowing integrin over a bare $\beta$-CD surface in the absence of dendrimer.
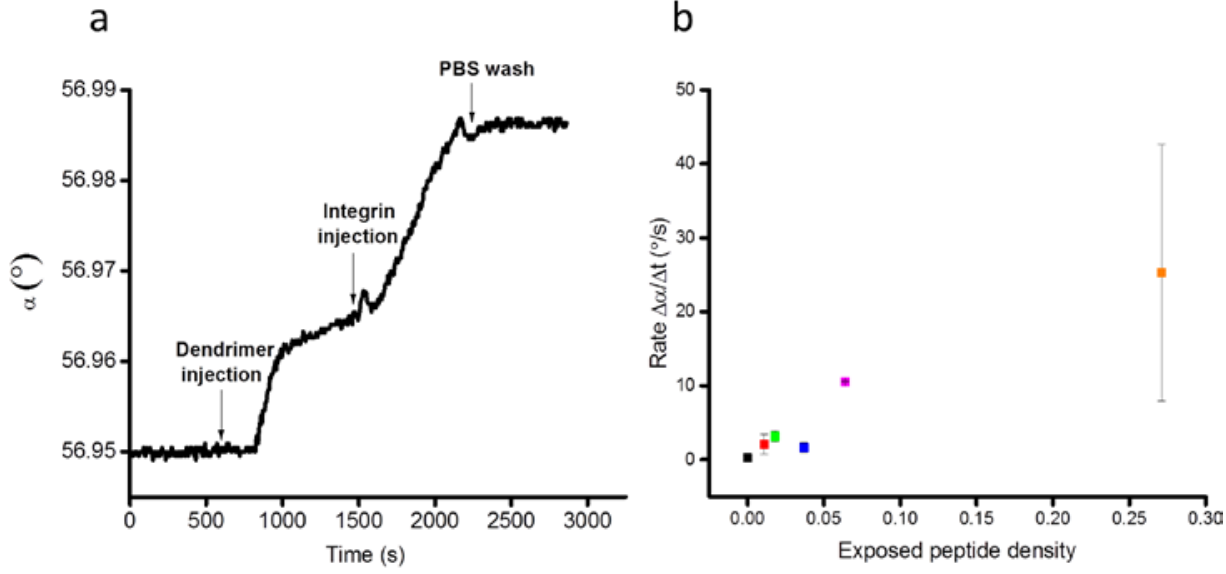

Figure 5.2 - a) Typical SPR plot of G0-(FY) $)_{4}$ showing the steps involved in studying the integrin adsorption on a dendrimer-functionalized surface. b) The rate of the SPR angle change during integrin adsorption step is plotted as a function of exposed peptide density (per $\beta$-CD cavity). Data points, G3-(FY) 30 (orange), G6-(YFY) $)_{53}$ (blue), G2-(FY) 15 (magenta), G0.5-(FY) 7 (green), $\mathrm{GO}-(\mathrm{FY})_{4}$ (red) and bare $\beta$-CD (black). Error bars represent standard error from two individual experiments. 
From the slope of the integrin adsorption step, the rate of adsorption was calculated. For all dendrimers, the rate of adsorption was plotted as a function of the exposed peptide density as shown in Figure 5.2b. A linear enhancement of the rate of integrin adsorption vs the exposed peptide density was observed. This suggests that by varying the exposed peptide density, the integrin adsorption can be controlled.

\subsubsection{Effect of exposed peptide density on the morphology of human dermal microvascular endothelial cells (HMVECs)}

As a next step, the effect of the variation of the exposed peptide density on the morphology of HMVECs was investigated. Bare $\beta$-CD substrates and surfaces coated with G0-(FY) 4 , G3-(FY) 30 and G6-(YFY) $)_{53}$ dendrimers and fibronectin (positive control) were fabricated on glass substrates (to avoid auto fluorescence and quenching that would result from using a gold substrate). These surfaces were incubated for $3 \mathrm{~h}$ in a solution of the HMVEC cells with approximately $2 \times 10^{5}$ cells per well. After fixation and permeabilization, the cells were stained for various morphological cues (details of the staining protocol are described in the Materials and methods section) and visualized by confocal laser scanning microscopy (CLSM).

The cells showed a normal, healthy morphology on bare $\beta$-CD and on the G0-(FY) 4 and G6-(YFY) 53 dendrimer-coated platforms. However, staining with actin and the focal adhesion protein vinculin (Figure 5.3a) revealed that the cell morphology on a surface with G3-(FY) $)_{30}$, which has the highest number of exposed peptide moieties per $\beta$-CD cavity, was more less spread when compared to HMVECs cultured on fibronectin or $\beta$ CD-coated surfaces. Moreover, the cells did not show the localization of focal adhesion protein vinculin at the end of the actin filaments indicating that the interaction between the cells and the surface is not integrin mediated. This behavior is attributed to the high exposed ligand density of the G3-(FY) $)_{30}$ dendrimer-coated substrate. Platforms with G3-(FY) 30 $_{30}$ dendrimer can expose $10^{13}$ peptides $\mathrm{cm}^{-2}$ while in the case of G0-(FY) 4 this number is only $4 \times 10^{11} \mathrm{~cm}^{-2}$ and for G6-(YFY) $)_{53}$ it is $7 \times 10^{11} \mathrm{~cm}^{-2}$. Literature has shown that when the ligand density of an RGD peptide was increased from $6 \times 10^{8}$ to 6 $\times 10^{11} \mathrm{~cm}^{-2}$, such a high density was detrimental for cell adhesion and spreading. ${ }^{17,18}$ It was argued that a high ligand density causes steric crowding of the peptides thus 
limiting the accessibility of the peptides to the cell surface receptors thus affecting the cell adhesion and spreading.

a
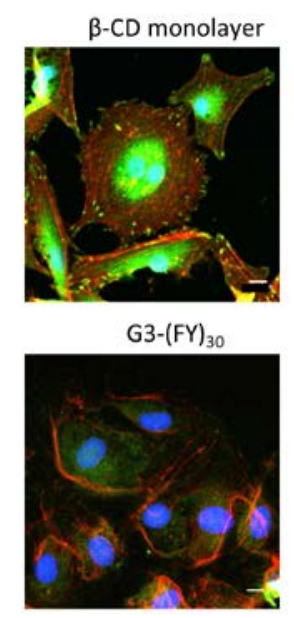

GO-(FY)

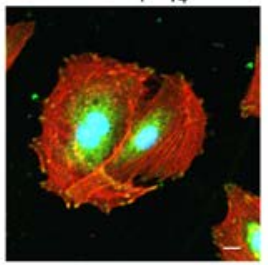

G6-(YFY) $)_{53}$

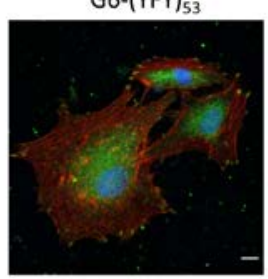

b

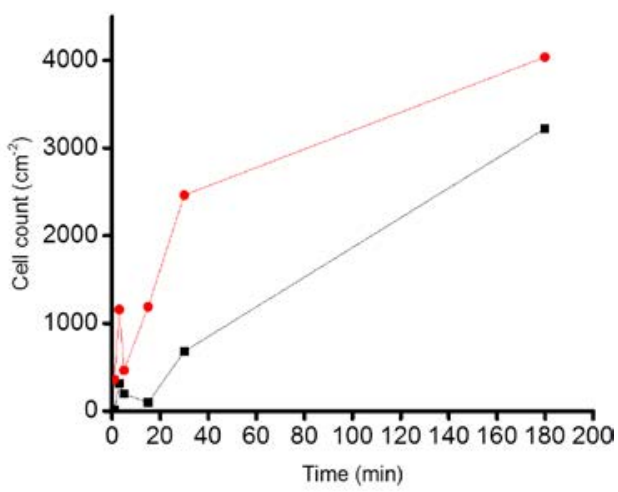

Figure 5.3 - a) Morphology attained by HMVECs on different surfaces after $3 \mathrm{~h}$ incubation studied by CLSM: actin (red), vinculin (green) and nuclei (blue) (scale bars $10 \mu \mathrm{m}$ ). b) Plot showing the adsorption kinetics of HMVECs on bare $\beta$-CD (black) and G6-(YFY) ${ }_{53}$ (red). The quantification was performed using the ImageJ software.

Next, we investigated the kinetics of the adhesion of HMVECs on these surfaces. We zoomed in on G6-(YFY) $)_{53}$ and bare $\beta-C D$ substrates for this study, because the morphology of the cells on the G3-(FY) $)_{30}$ surface was less efficient. The cells were incubated on G6-(YFY) $)_{53}$ and bare $\beta-\mathrm{CD}$ platforms at a predetermined cell concentration, and after each time point a sample was removed, rinsed, and stained for actin and DAPI. The cell density was determined from optical microscopy images and plotted as a function of time (Figure 5.3b). It was observed that in the $3 \mathrm{~h}$ incubation period there was a rapid increase in cell adhesion on the G6-(YFY) $)_{53}$ platform compared to the bare $\beta$-CD surface. After $3 \mathrm{~h}$, the morphology of the cells on each platform was studied by staining for actin, and the images showed a similar morphology in terms of shape and actin organization on both the surfaces with marginal difference in number of adhered cells. 


\subsubsection{Effect of variation in exposed peptide density on VEGF-A signaling}

Next the effect of the dendrimer platforms on eliciting VEGF-A signaling was assessed (Figure 5.4a,b). The HMVEC cells were incubated on $\beta-\mathrm{CD}$, G0-(FY) ${ }_{4}$, G3-(FY) ${ }_{30}$ and G6-(YFY) 53 platforms for $3 \mathrm{~h}$, and subsequently fixated and stained with antibodies specific for $\alpha 9 \beta 1$ integrin and phospho-VEGFR2 to check for integrin expression and VEGF-A activation. We used the $\alpha 9 \beta 1$ integrin antibody at a concentration of $20 \mu \mathrm{g} / \mathrm{ml}$ in TBP (Triton-X in PBS) at $4^{\circ} \mathrm{C}$ overnight followed by incubation with the secondary antibody as reported in literature. ${ }^{19}$ The hallmark for VEGF activation is the phosphorylation of tyrosine 1175 (pY-1175) at the VEGFR-2 receptor. ${ }^{20,16}$ Quantification of protein expression was done from total cell fluorescence from antibody staining as reported previously. ${ }^{21,22}$

Significant integrin staining was observed on the surface of HMVECs cultured on the $\beta$ $\mathrm{CD}$ platform (Figure 5.4b). The $\beta$-CD molecule is known to be an inhibitor of the caveolae/raft dependent $\beta 1$-integrin internalization. ${ }^{23,24}$ It interacts with the $\beta 1$-integrin on the cell surface, but instead of activating it for subsequent cascades of reactions, it inhibits its endocytosis. This could be the reason that integrin staining was observed at the cell periphery on bare $\beta$-CD substrates. In the case of the G6-(YFY) 53 platform, the majority of the integrins are supposedly endocytosed leading to less staining on the periphery of the cell. Moreover, literature also suggest that VEGFR-2 receptors, which are the primary cognate receptors for VEGF activation, localize in the caveolae, thus disruption of these caveolae by $\beta$-CD in the monolayer might explain the observed decreased VEGF activity compared to the dendrimer surfaces. ${ }^{25}$ This observation was useful in the sense that higher VEGF activation for the G6-(YFY) ${ }_{53}$ dendrimer surface can be attributed to the functional interaction between the peptide and the integrins leading to activation of VEGF which is not possible on a bare $\beta$-CD platform. 

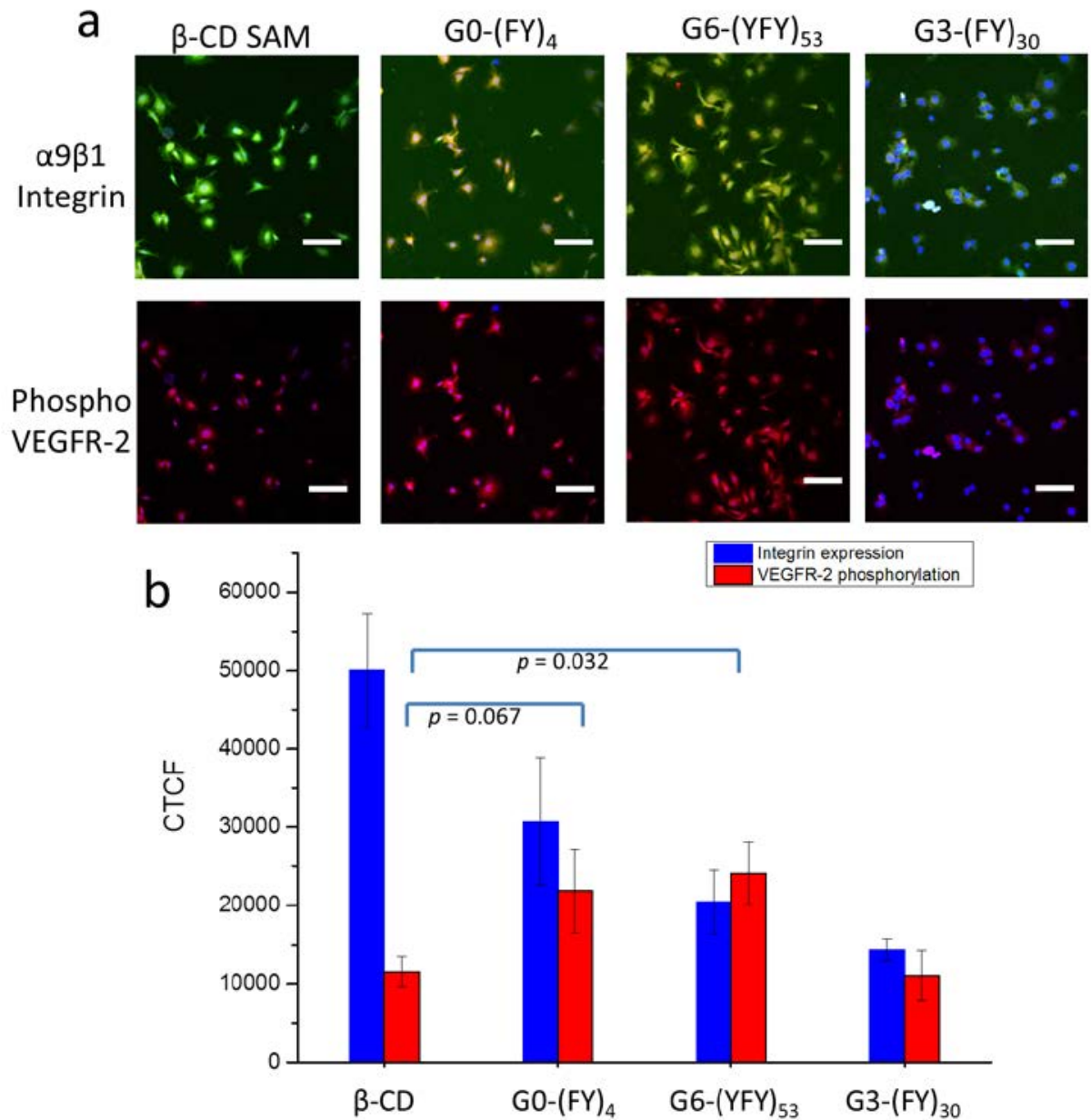

Figure 5.4 - a) Confocal laser scanning microscopy images of $\alpha 9 \beta 1$ integrin (green), phospho VEGFR-2 (pY-1175) (pink), and nuclei (blue) of HMVEC cells cultured on $\beta$-CD (control), G0$(\mathrm{FY})_{4}$, G3-(FY) $)_{30}$ and G6-(YFY) $)_{53}$ substrates, shown in the order of increasing exposed peptide density. Upper panel shows integrin + pY-1175 merged while lower panel shows only pY1175 staining (Scale bars $20 \mu \mathrm{m}$ ). b) Corrected total cell fluorescence (CTCF) intensities calculated as reported before. ${ }^{23}$ Error bars represent a single standard deviation, $p$ values were calculated from a student t-test $(n=70)$.

Although in the case of G0-(FY) 4 slightly higher integrin staining was observed compared to G6-(YFY) $)_{53}$, which can be attributed to the relatively lower endocytosis 
rate compared to G6-(YFY) $)_{53}$, the VEGFR-2 phosphorylation levels were found to be similar. In the case of the G3-(FY) $)_{30}$ dendrimer, due to the very high exposed ligand density, cells showed no focal adhesion formation suggesting suboptimal contact of the cells with the substrate, thus explaining the observed very low integrin expression. These observations suggest that the cells can respond to either the presence or the absence of functional ligands at the $\beta$-CD SAM. An optimal density of these peptides is essential to trigger VEGF phosphorylation as a too high density is detrimental for cell adhesion.

To further investigate the importance of the presence of $\alpha 9 \beta 1$ integrin receptors on the surface of the cells for VEGF activation, the human umbilical vein endothelial cell (HUVEC) type was chosen because it is known to express very few $\alpha 9 \beta 1$ integrin receptors. ${ }^{7}$ G6-(YFY) $)_{53}$-coated and bare $\beta$-CD substrates were incubated with HUVECs with an approximate cell seeding concentration of $2 \times 10^{5}$ cells $/ \mathrm{ml}$, while another substrate with G6-(YFY) $)_{53}$ was incubated with approximately the same density of HMVECs. After incubation for $3 \mathrm{~h}$, the cells were fixated and stained for both the integrin and the VEGFR-2 phosphorylation antibody and imaged using fluorescence microscopy, as shown in Figure 5.5.

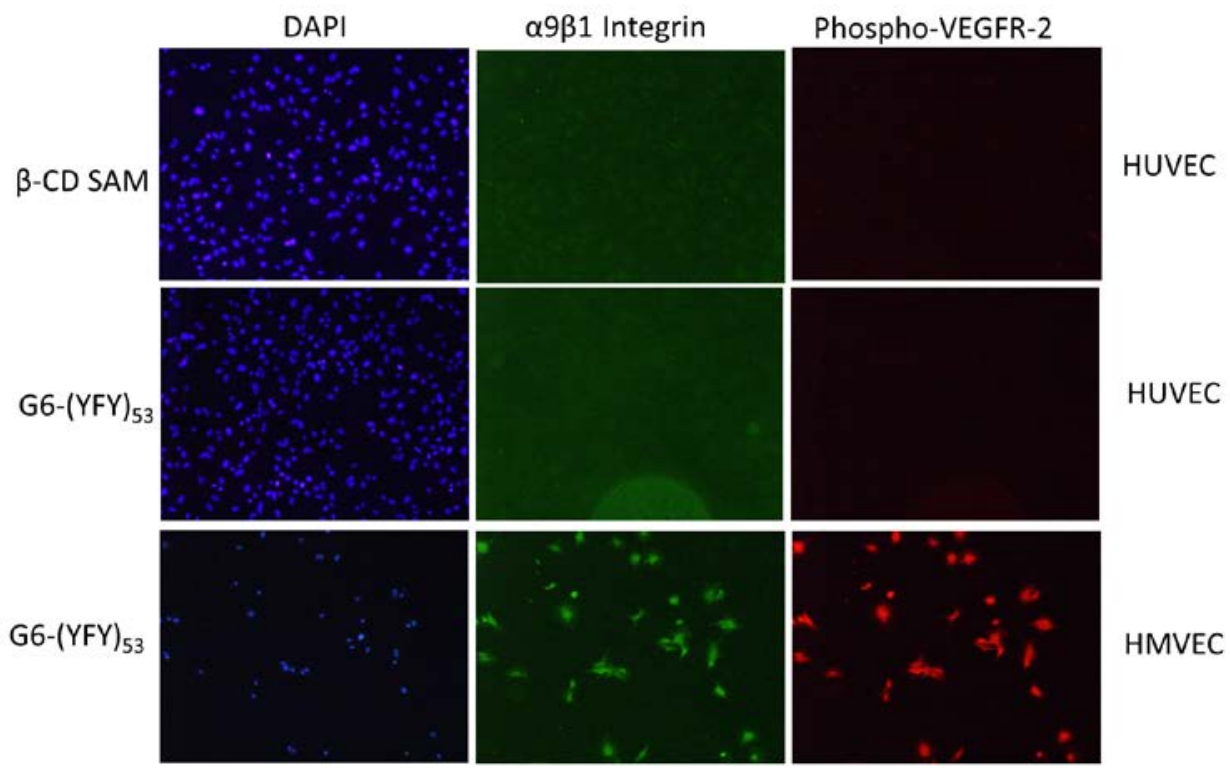


Figure 5.5 - Fluorescence microscopy images showing staining for $\alpha 9 \beta 1$ integrin and VEGFR-2 phosphorylation (pY-1175) in HUVEC and HMVEC cell lines adsorbed onto dendrimer-coated or bare $\beta$-CD surfaces (Green: $\alpha 9 \beta 1$ integrin; red: pY-1175; blue: DAPI).

Significant HUVEC cell adhesion was observed on the G6-(YFY) $)_{53}$ and $\beta$-CD platforms. However, staining was observed neither for $\alpha 9 \beta 1$ integrin nor for pY-1175, in contrast to HMVECs. This indicates that the specific $\alpha 9 \beta 1$ integrin receptor clustering observed for HMVECs is crucial for VEGF-A activity.

\subsubsection{VEGF-mediated angiogenesis induced by dendrimer-coated platforms}

After the cell experiments, these platforms were further extended to investigate the VEGF-A triggering activity in an in vivo model. There is a wide application of VEGF in supplying a pro-angiogenic signal to treat ischemic heart disease. ${ }^{26}$ But the conventional approach to deliver growth factors from the solution phase is costly and requires serial doses of expensive growth factors to maintain a therapeutic level. ${ }^{14,27}$ We envisioned that our G6-(YFY) 53 platform might be able to induce integrin clustering in vivo, to trigger the activity of VEGF present locally without any exogenous supply of growth factor.

In order to study the angiogenic property of our dendrimer platform, the chicken chorioallantoic membrane (CAM) assay was employed, as a representative model for investigating VEGF mediated angiogenesis. ${ }^{14} \mathrm{CAM}$ is formed by the fusion of the chorion and the allantoic membrane. It is characterized by a highly dense network of arteries and veins. In order to evaluate the angiogenic potential of the dendrimer platform, it has been applied on top of a pre-formed CAM, and the capillary perfusion as well as the formation of new capillaries were monitored for two days. Substrate preparation for the CAM experiments was the same as described previously for the cell experiments. First, the angiogenesis potential of the dendrimer was investigated in solution (Figure 5.6). A solution of $40 \mu \mathrm{g} / \mathrm{ml}$ of G6-(YFY) ${ }_{53}$ dendrimer and $100 \mathrm{ng} / \mathrm{ml}$ of VEGF in sterile PBS buffer was prepared. As a negative control we used PBS alone, while a solution of VEGF (100 ng/ml) functioned as a positive control. Images of CAM exposed to these solutions were taken on the $0^{\text {th }}$ and $2^{\text {nd }}$ day using a portable USB microscope (Veho VMS-001). A distance between the microscope and the egg was 
maintained to visualize the whole egg. Difference between the capillary density enhancement between the $0^{\text {th }}$ and $2^{\text {nd }}$ day in each sample is used as an indication of angiogenesis. Each experiment was repeated three times.

a

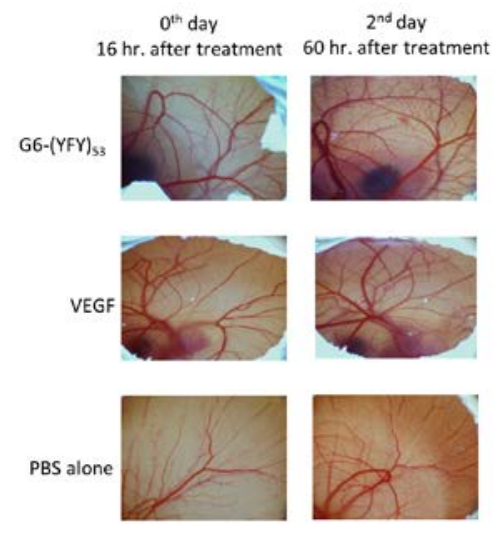

b

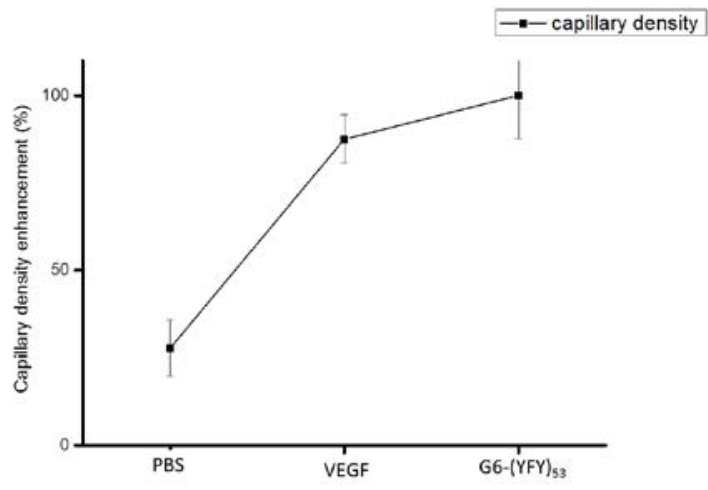

Figure 5.6 - a) Representative en face images of the surface of a CAM at the $0^{\text {th }}$ and $2^{\text {nd }}$ day of incubation with G6-(YFY) $)_{53}$ dendrimer $(40 \mu \mathrm{g} / \mathrm{ml})$, VEGF (100 ng/ml), PBS (carrier medium only). (error bars represent a single standard deviation) $(n=3)$. b) Quantification of the capillary density enhancement on the $2^{\text {nd }}$ day compared to the $0^{\text {th }}$ day of the CAM surfaces.

A 65\% higher angiogenic activity of the dendrimer solution was observed compared to the carrier solution (PBS). Therefore, next this dendrimer was immobilized on a $\beta-C D$ surface to investigate the potential of the dendrimer-coated platform to locally trigger the angiogenic activity. The dendrimer platform was placed upside down on the CAM membrane (Figure 5.7). 

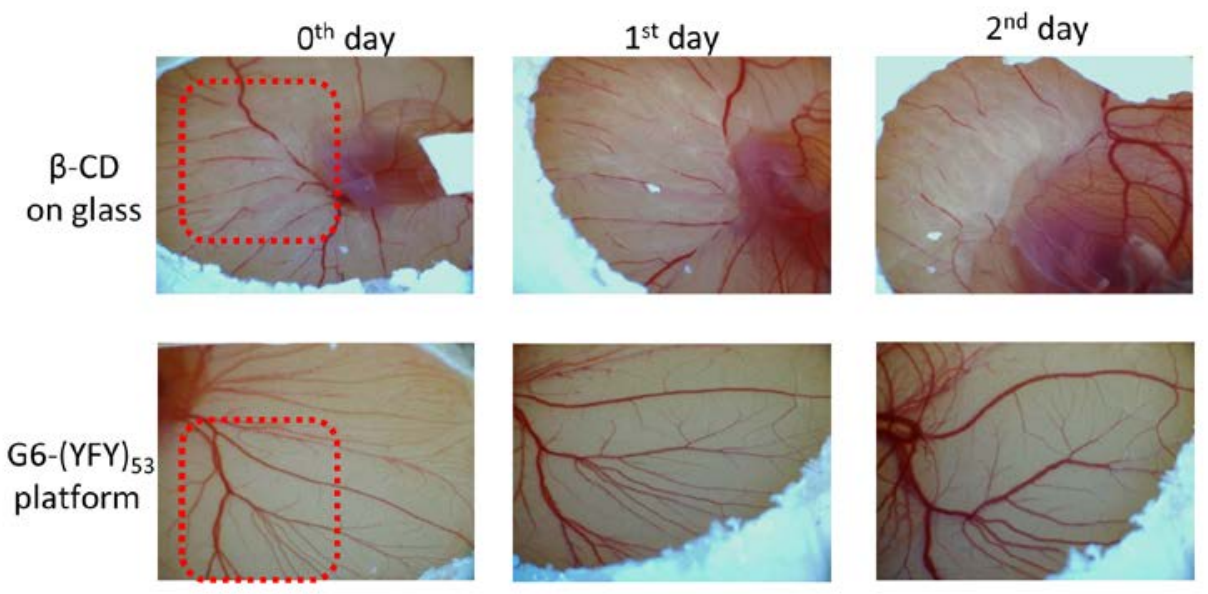

Figure 5.7 - Representative en face images of the surface of CAM on the $0^{\text {th }}, 1^{\text {st }}$ and $2^{\text {nd }}$ day upon contacting the CAM at the dotted areas with substrates containing bare $\beta$-CD or G6-(YFY) 53- $^{-}$ coated substrates. Marked areas in the images show the placement site of the substrates

The negative control, a bare $\beta$-CD surface, showed a decrease in the capillary density with time (Figure 5.7). This can be attributed to a diminished VEGF activity on the $\beta$ CD surface due to a caveolae-dependent VEGF inhibition as discussed previously. In contrast, when G6-(YFY) $)_{53}$ was immobilized on the $\beta$-CD surface, an increase in the capillary density and perfusion was observed on the consecutive days compared to the initial treatment $\left(0^{\text {th }}\right.$ day).

To gain further understanding of the physiology of the capillaries after exposing them to the platforms, the area of the CAM exposed to a platform was subjected to immunohistochemistry analysis. Details of the immunohistochemistry technique is given in the Materials and methods section. The analysis revealed that the CAM samples exposed to a G6-(YFY) $)_{53}$ substrate or a positive control of VEGF in solution showed the formation of a well-defined capillary epithelium, which is an indication of normal development of the capillary (Figure 5.8). ${ }^{28}$ In the case of a $\beta$-CD substrate exposed to the CAM surface, the capillary epithelium formation was found to be rudimentary. Moreover, in the case of the G6-(YFY) 53 and VEGF samples, staining of the CD-31 antibody representing VEGF activity was observed in the inner lining of the 
capillaries, while fewer staining sites were observed in the case of the $\beta$-CD exposed capillaries. $^{29,30}$

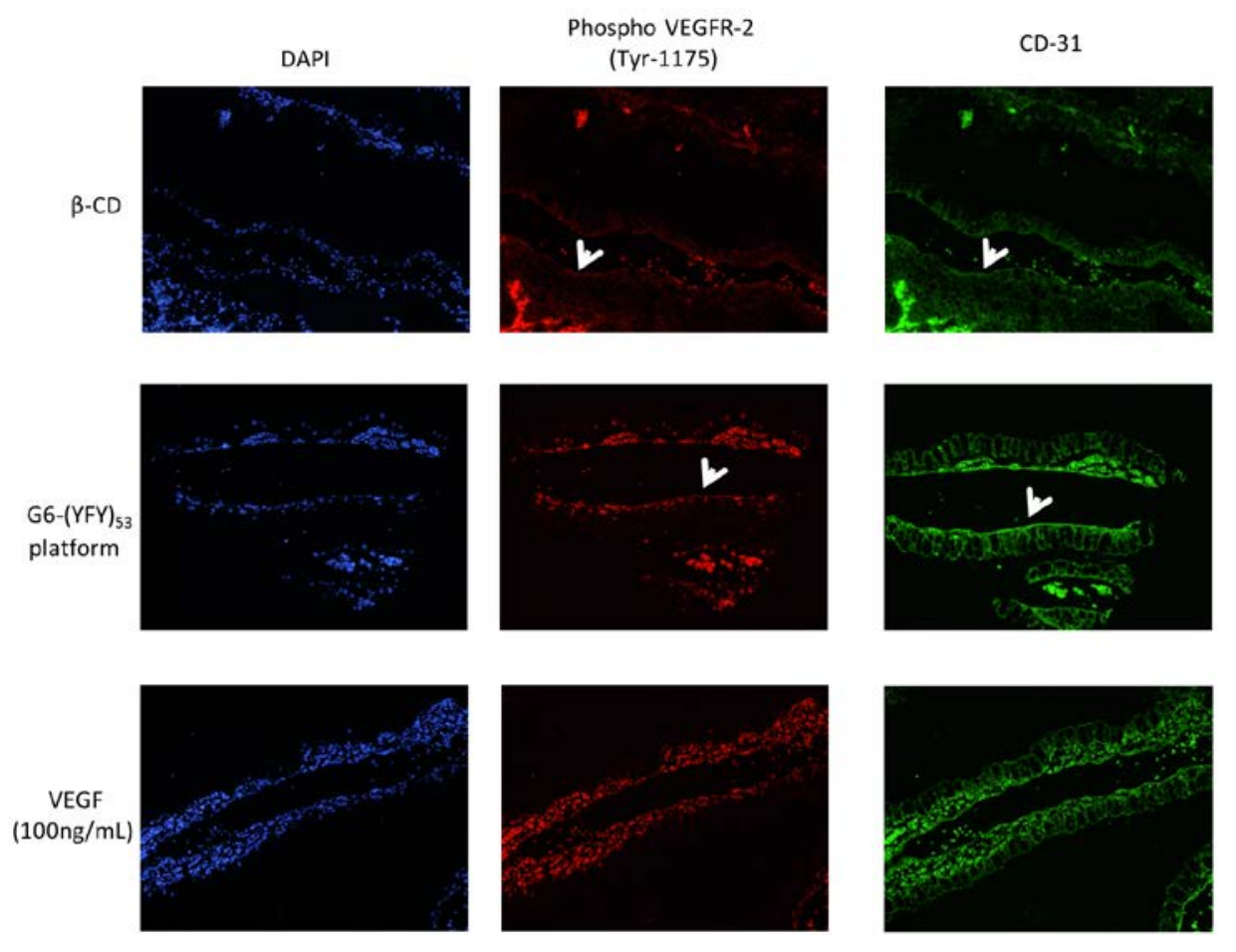

Figure 5.8 - Fluorescence immunohistochemistry images of the capillaries exposed to the various surfaces as indicated. Arrowheads show the staining of the CD-31 antibody on the inner walls of the capillaries (image area: $877 \mu \mathrm{m} \times 660 \mu \mathrm{m}$ ).

\subsubsection{Towards advanced biomedical application}

To demonstrate the potential biomedical application of our supramolecular system, the fabrication of the entire platform on a co-polymer of poly(trimethylene carbonate) crosslinked with $\varepsilon$-caprolactone (PTMC-CL) was carried out. PTMC is known to be inert, biocompatible and biodegradable with proven biomedical applications. ${ }^{31-33}$ The steps involved in the fabrication of the $\beta$-CD monolayer on this polymer are shown in Figure 5.9. Briefly, the polymer substrate was first rinsed with ethanol, dried under nitrogen flow and subjected to an oxygen plasma treatment for $10 \mathrm{~s}$ to generate aldehyde groups ${ }^{34}$ on the surface of the polymer. Immediately after oxygen plasma 
treatment, the polymer was incubated in an ethanolic solution of $\beta-C D$ amine ${ }^{17}$ for $1 \mathrm{~h}$. Subsequently, the polymer substrate was treated with a sodium cyano-borohydrate $\left(\mathrm{NaBH}_{4} \mathrm{CN}\right)$ solution in PBS containing $4 \%$ of ethanol to convert the initially formed and intrinsically reversible imine bonds into stable amines. Each step of the functionalization process was checked by contact angle measurements.

Bare PTMC without any treatment was hydrophobic as indicated by a high contact angle $\left(100^{\circ}\right)$. After oxygen plasma treatment, the surface showed a lower contact angle $\left(31^{\circ}\right)$, as generation of the aldehyde groups made the surface more hydrophilic. Coupling of $\beta$-CD-amine to the PTMC polymer followed by chemical reduction and extensive washing led to an increase of the contact angle to $54^{\circ}$ indicating the formation of a $\beta$-CD monolayer. The surface functionalization was further characterized by X-ray photoelectron spectroscopy (XPS) (Figure 5.10). After $\beta$-CD coupling, an increase in the nitrogen contents of the polymer was observed from approx. $1 \%$ for unfunctionalized and oxidized PTMC to $9 \%$ for $\beta$-CD-attached PTMC, which confirmed the attachment of $\beta$-CD-amine to the PTMC surface.

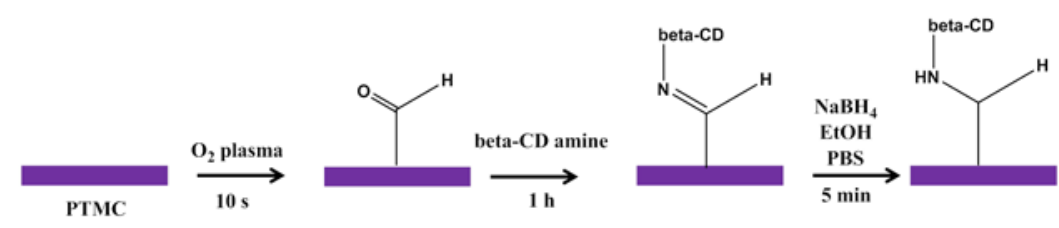

Figure 5.9 - Scheme showing the steps involved in the fabrication of a $\beta$-CD monolayer on a PTMC polymer substrate. 

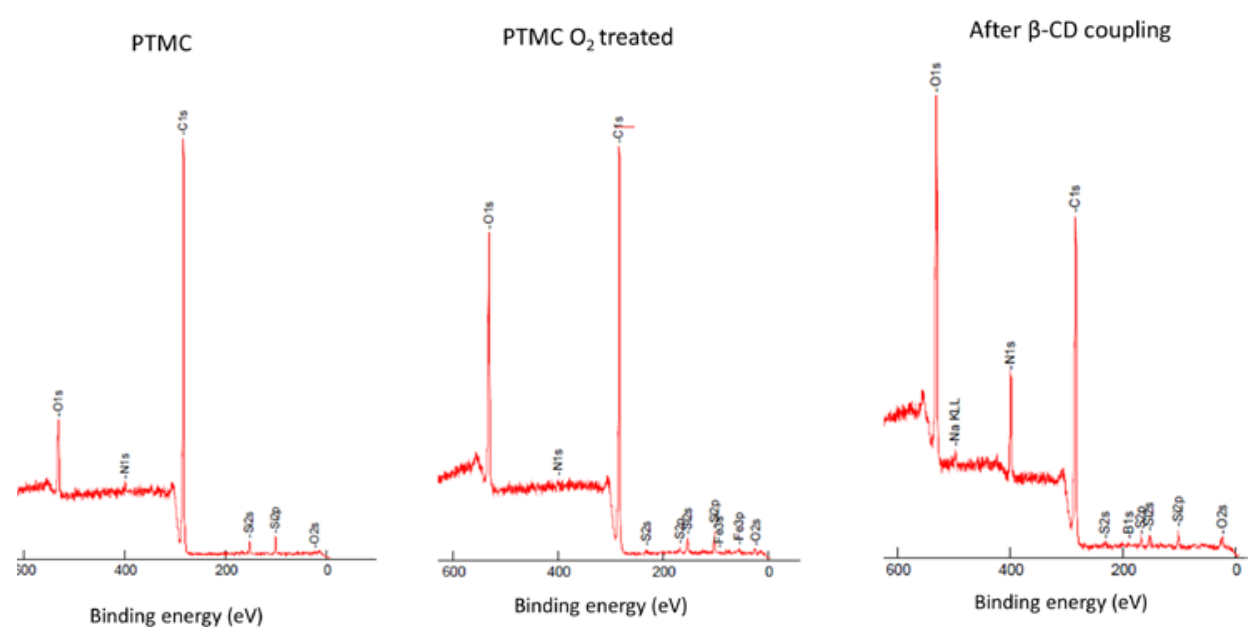

Figure 5.10 - XPS spectra showing changes in elemental composition after each fabrication step shown in Figure 5.10.

After fabrication and characterization of the $\beta$-CD monolayer, G6-(YFY) $)_{53}$ was assembled on the functionalized polymer film. Thereafter, these substrates were incubated with HMVEC cells. The HMVECs exhibited a comparable morphology on the dendrimer-coated polymer substrates as observed before on the gold and glass surfaces. Thus, we envision that our supramolecular dendrimer system can be extended to its fabrication on functional biopolymer substrates to achieve a dynamically displaying bioactive ligands which can support the immense potential of biodegradable polymers in sustained release and other therapeutic applications. ${ }^{35-37}$

\subsection{Conclusions}

The exposed peptide density exhibited by dendrimer fabrication on $\beta$-CD SAMs has been shown here to control integrin clustering and VEGF activation both in vitro and in vivo. The adhesion of pure integrin receptors was found to be strictly dependent on the variation in peptide density as observed from SPR experiment. This density dependence was also seen in morphology study with HMVEC cells, where surfaces with too high peptide density (G3-(FY) $)_{30}$ ) was found to be detrimental to the cell spreading. This integrin clustering was found to trigger VEGF activation in vitro. We found that 
presence of $\alpha 9 \beta 1$ integrin receptors was crucial for VEGF activation, as in case of HUVEC cell line no VEGF activation was observed due to very low $\alpha 9 \beta 1$ integrin expression. Our platforms also induced VEGF mediated angiogenesis under in vivo condition (CAM assay). Moreover by fabricating the entire platform on the biomedically relevant PTMC, it is possible to exploit this strategy for advance biomedical applications. Thus, the approach promises the spatio-temporal control over growth factor activity without the need for an exogenous supply of VEGF, and suggests the use of this approach in ischemic heart diseases where new blood vessel development is required in infarcted tissue area.

\subsection{Acknowledgments}

Dr. Nicole Georgi and Jenny Brinkmann are acknowledged for their help with the CAM assay and immunohistochemistry. Dr. Wies van Roosmalen is acknowledged for her help with the cell experiments. Dr. Erhan Bat is acknowledged for providing the PTMC polymer.

\subsection{Materials and Methods}

General. Chemicals were obtained from commercial sources and are used as received. Pure integrin $\alpha 9 \beta 1$ was obtained from R\&D systems (catalogue number 5438-A9). HMVEC and HUVEC cells was purchased from Lonza along with EBM-2 medium for cell culture. Antibiotic treatment free white leghorn eggs was purchased from Het Anker BV, Ochten. Staining antibodies for $\alpha 9 \beta 1$ integrin was purchased from Biolegend, phospho-VEGFR-2 (pY-1175), FITC-vinculin, phalloidin alexa-568 was purchased from cell signaling, DAPI was purchased from Sigma-Aldrich respectively.

\section{HMVEC/HUVEC cell culture}

As received, HMVEC cells were stored in $-80^{\circ} \mathrm{C}$ under nitrogen environment and taken out as and when required. T-75 flask was used for cell culture and coated with $10 \mu \mathrm{g} / \mathrm{ml}$ solution of fibronectin in sterile PBS for $1 \mathrm{~h}$ at $37{ }^{\circ} \mathrm{C}$ under $\mathrm{CO}_{2}$ incubator. After fibronectin coating of flask, it was washed 3 times with the culture medium. EBM-2 
medium was mixed with all the supplementary nutrients provided with the bullet kit (except VEGF-A) along with $10 \%$ FBS to constitute the final culture medium. Even though $10 \%$ FBS was added during cell culture, only $1 \%$ FBS was added to medium prepared for seeding the cells on dendrimers surface, as large excess of FBS in the medium can form a layer on the substrate diminishing the effect of active ligand. Afterwards the flask with $14 \mathrm{ml}$ of culture medium was incubated for $15 \mathrm{~min}$. and vial containing pellets of HMVECs was thawed and content of vial was transferred to the flask. After $1^{\text {st }}$ day the medium was changed with the fresh medium to remove DMSO in the medium (DMSO is the component of freezing media used to freeze cells). After $1^{\text {st }}$ day medium is changed every 2 days and cells were passaged every time they reached confluence. Cells are always used between passage 3 and 6. VEGF-A was always added freshly to the medium containing cells when they were ready for seeding on the substrates. VEGF-A concentration used was $10 \mathrm{ng} / \mathrm{ml}$ of medium to mimic its concentration in the natural environment. HUVEC cell culture was done using same protocol.

\section{Substrate preparation for cell experiments}

Dendrimers were stored in methanol for prolonged storage at a $1 \mathrm{mg} / \mathrm{ml}$ concentration. After the fabrication of $\beta$-CD monolayer on the glass, ${ }^{38}$ they were kept in the sterile atmosphere under nitrogen. Before experiments $\beta$-CD surface was rinsed three times with $70 \%$ ethanol and dried under nitrogen flow. Afterwards they are incubated with each dendrimer solution. After complete evaporation of methanol, platform was incubated with similar volume of PBS for at least $12 \mathrm{~h}$ to assure their proper immobilization on $\beta$-CD surface. Next day they were removed and again washed three times with milliQ water and transferred to the well plates. In the well plates they are again washed shortly with sterile PBS followed by 70 \% ethanol followed by two times with PBS.

\section{Staining procedure in morphology study}

Dendrimer platform was incubated with the HMVEC cells in approximately $2 * 10^{5}$ cells per well for $3 \mathrm{~h}$. Afterwards platform was removed and washed with the PBS 
buffer and fixated with buffered formaldehyde solution for 10 min. followed by three times washing with the PBS buffer. Then the cells were permeabilized with $0.1 \%$ triton-X in PBS solution with $250 \mathrm{mg}$ of bovine serum albumin (TBP) for $2 \mathrm{~h}$ at room temperature. Substrate was then incubated with vinculin-FITC in dilution of 1:50 in TBP solution at $4^{\circ} \mathrm{C}$ overnight. Next day the substrate was washed three times with TBP solution and incubated with Phalloidin-Alexa 568 in 1:100 dilution for $1 \mathrm{~h}$ at room temperature. Followed by two times washing with TBP and one time washing with the PBS and final incubation with DAPI in 1:100 dilution in PBS for $10 \mathrm{~min}$. Afterwards platform was washed with PBS and mounted with mounting medium and visualized for staining under confocal laser scanning microscope.

\section{CAM assay procedure}

In ovo CAM assay method is used to analyze the angiogenesis potential of G6-(YFY) $)_{53}$ dendrimer in the solution phase. Fresh the eggs as received are immediately transferred to humidified incubator at $37{ }^{\circ} \mathrm{C}$ for hatching. After 3 days using syringe with a very small needle, approximately 3-4 $\mathrm{ml}$ of albumen is removed from the egg using the protocol reported in the literature. ${ }^{39}$ Then the window of approx. 3" x 3" dimension was made in to the egg and both window and needle puncture point is closed using plastic adhesive tape. Eggs are further incubated for next 3 days. On $6^{\text {th }}$ day sample treatment is made and monitored further for the next 3 days. Quantification of the capillary density enhancement is done using reported procedure. ${ }^{40}$

\section{Immunohistochemistry (IHC)}

The embryo was sacrificed on $9^{\text {th }}$ day and CAM surface was flooded with neutral formalin solution for 14-16 h. After $16 \mathrm{~h}$ CAM became thick and was excised from the surface and dehydrated completely with sequential incubation with the ethanol with increasing concentration in water followed by paraffin embedding using standard technique. The paraffin embedded tissues are then sectioned in to the slices (approx. 3$10 \mu \mathrm{m}$ thick) using microtome. Sectioning was done in such a manner so as to get the sections with varied morphology. After mounting the sections on the clean glass slides coated with gelatin, sections are prepared for de-paraffinization and rehydration using 
incubation of slides in xylene followed by decreasing concentration of ethanol. After rehydration, endogenous peroxidase activity inhibition was done by incubating the slides with $3 \% \mathrm{H}_{2} \mathrm{O}_{2}$ solution in methanol at room temperature for $10 \mathrm{~min}$. followed by heat induced antigen retrieval in Tris/EDTA buffer at $95-100{ }^{\circ} \mathrm{C}$ for $10 \mathrm{~min}$. After antigen retrieval step slide was washed three times with PBS and then antibody staining was carried out in same way as we described in cell experiments. Antibody used for staining CAM sections was CD-31 (PECAM-1), and nuclei were stained with DAPI.

\subsection{References}

1. Kiessling, L.L., Gestwicki, J.E. \& Strong, L.E. Synthetic multivalent ligands as probes of signal transduction. Angewandte Chemie International Edition 45, 2348-2368 (2006).

2. Kiessling, L.L. \& Grim, J.C. Glycopolymer probes of signal transduction. Chemical Society Reviews 42, 4476-4491 (2013).

3. Shan, M. Nonsteroidal bivalent estrogen ligands: an application of the bivalent concept to the estrogen receptor. ACS Chemical Biology 8, 707-715 (2013).

4. Taipale, J. \& Keski-Oja, J. Growth factors in the extracellular matrix. The FASEB Journal 11, 51-59 (1997).

5. Ferrara, N., Gerber, H. P. \& LeCouter, J. The biology of VEGF and its receptors. Nature Medicine 9, 669-676 (2003).

6. Olsson, A.K., Dimberg, A., Kreuger, J. \& Claesson-Welsh, L. VEGF receptor signalling ? in control of vascular function. Nat Rev Mol Cell Biol 7, 359-371 (2006).

7. Oommen, S., Gupta, S.K. \& Vlahakis, N.E. Vascular endothelial growth factor a (VEGF-A) induces endothelial and cancer cell migration through direct binding to integrin $\alpha 9 \beta 1$ : identification of a specific $\alpha 9 \beta 1$ binding site. Journal of Biological Chemistry 286, 1083-1092 (2011).

8. Chen, T.T. Anchorage of VEGF to the extracellular matrix conveys differential signaling responses to endothelial cells. The Journal of Cell Biology 188, 595609 (2010). 
9. Cabanas-Danes, J., Huskens, J. \& Jonkheijm, P. Chemical strategies for the presentation and delivery of growth factors. Journal of Materials Chemistry B 2, 2381-2394 (2014).

10. Li, L., Klim, J.R., Derda, R., Courtney, A.H. \& Kiessling, L.L. Spatial control of cell fate using synthetic surfaces to potentiate TGF- $\beta$ signaling. Proceedings of the National Academy of Sciences 108, 11745-11750 (2011).

11. Hudalla, G.A., Koepsel, J.T. \& Murphy, W.L. Surfaces that sequester serumborne heparin amplify growth factor activity. Advanced Materials 23, 54155418 (2011).

12. Stabley, D., Retterer, S., Marshall, S. \& Salaita, K. Manipulating the lateral diffusion of surface-anchored EGF demonstrates that receptor clustering modulates phosphorylation levels. Integrative Biology 5, 659-668 (2013).

13. Manz, B.N., Jackson, B.L., Petit, R.S., Dustin, M.L. \& Groves, J. T-Cell triggering thresholds are modulated by the number of antigen within individual t-cell receptor clusters. Proceedings of the National Academy of Sciences 108, 9089-9094 (2011).

14. Webber, M.J. Supramolecular nanostructures that mimic VEGF as a strategy for ischemic tissue repair. Proceedings of the National Academy of Sciences 108, 13438-13443 (2011).

15. Boekhoven, J., Rubert Pérez, C.M., Sur, S., Worthy, A. \& Stupp, S.I. Dynamic display of bioactivity through host-guest chemistry. Angewandte Chemie International Edition, 52, 12077-12080 (2013).

16. Tugues, S. Tetraspanin CD63 promotes vascular endothelial growth factor receptor 2- $\beta 1$ integrin complex formation, thereby regulating activation and downstream signaling in endothelial cells in vitro and in vivo. Journal of Biological Chemistry 288, 19060-19071 (2013).

17. Le Saux, G., Magenau, A., Böcking, T., Gaus, K. \& Gooding, J.J. The relative importance of topography and RGD ligand density for endothelial cell adhesion. PLoS ONE 6, e21869 (2011).

18. Deeg, J.A. \& Spatz, J. Impact of local versus global ligand density on cellular adhesion. Nano Letters 11, 1469-1476 (2011).

19. Lafuste, P. ADAM12 and $\alpha 9 \beta 1$ integrin are instrumental in human myogenic cell differentiation. Molecular Biology of the Cell 16, 861-870 (2005). 
20. Holmqvist, K. The adaptor protein shb binds to tyrosine 1175 in vascular endothelial growth factor (VEGF) receptor-2 and regulates VEGF-dependent cellular migration. Journal of Biological Chemistry 279, 22267-22275 (2004).

21. Burgess, A. Loss of human greatwall results in G2 arrest and multiple mitotic defects due to deregulation of the cyclin b-cdc2/pp2a balance. Proceedings of the National Academy of Sciences 107, 12564-12569 (2010).

22. Potapova, T.A., Sivakumar, S., Flynn, J.N., Li, R. \& Gorbsky, G.J. Mitotic progression becomes irreversible in prometaphase and collapses when wee1 and CDC25 are inhibited. Molecular Biology of the Cell 22, 1191-1206 (2011).

23. Du, J. Integrin activation and internalization on soft ECM as a mechanism of induction of stem cell differentiation by ECM elasticity. Proceedings of the National Academy of Sciences 108, 9466-9471 (2011).

24. Yancey, P.G. Cellular cholesterol efflux mediated by cyclodextrins: demonstration of kinetic pools and mechanism of efflux. Journal of Biological Chemistry 271, 16026-16034 (1996).

25. Cho, C. H. Localization of VEGFR-2 and PLD2 in endothelial caveolae is involved in VEGF-induced phosphorylation of MEK and ERK. American Journal of Physiology - Heart and Circulatory Physiology 286, H1881-H1888 (2004).

26. Gupta, R., Tongers, J. \& Losordo, D.W. Human studies of angiogenic gene therapy. Circulation Research 105, 724-736 (2009).

27. Post, M.J., Laham, R., Sellke, F.W. \& Simons, M. Therapeutic angiogenesis in cardiology using protein formulations. Cardiovascular Research 49, 522-531 (2001).

28. Seidlitz, E., Korbie, D., Marien, L., Richardson, M. \& Singh, G. Quantification of anti-angiogenesis using the capillaries of the chick chorioallantoic membrane demonstrates that the effect of human angiostatin is age-dependent. Microvascular Research 67, 105-116 (2004).

29. Cristina, C. VEGF and CD31 association in pituitary adenomas. Endocr Pathol 21, 154-160 (2010).

30. Mineo, T.C. Prognostic impact of VEGF, CD31, CD34, and CD105 expression and tumour vessel invasion after radical surgery for ib-iia non-small cell lung cancer. Journal of Clinical Pathology 57, 591-597 (2004). 
31. Bat, E., van Kooten, T.G., Feijen, J. \& Grijpma, D.W. Macrophage-mediated erosion of gamma irradiated poly(trimethylene carbonate) films. Biomaterials 30, 3652-3661 (2009).

32. Bat, E. \& Grijpma, D.W. Ultraviolet light crosslinking of poly(trimethylene carbonate) for elastomeric tissue engineering scaffolds. Biomaterials 31, 86968705 (2010).

33. Zhang, Z., Kuijer, R., Bulstra, S.K., Grijpma, D.W. \& Feijen, J. The in vivo and in vitro degradation behavior of poly(trimethylene carbonate). Biomaterials 27, 1741-1748 (2006).

34. Hosseini, S., Ibrahim, F., Djordjevic, I. \& Koole, L.H. Recent advances in surface functionalization techniques on polymethacrylate materials for optical biosensor applications. Analyst 139, 2933-2943 (2014).

35. Soppimath, K.S., Aminabhavi, T.M., Kulkarni, A.R. \& Rudzinski, W.E. Biodegradable polymeric nanoparticles as drug delivery devices. Journal of Controlled Release 70, 1-20 (2001).

36. Jeong, B., Choi, Y.K., Bae, Y.H., Zentner, G. \& Kim, S.W. New biodegradable polymers for injectable drug delivery systems. Journal of Controlled Release 62, 109-114 (1999).

37. Nair, L.S. \& Laurencin, C.T. Biodegradable polymers as biomaterials. Progress in Polymer Science 32, 762-798 (2007).

38. Onclin, S., Mulder, A., Huskens, J., Ravoo, B.J. \& Reinhoudt, D.N. Molecular printboards: monolayers of $\beta$-cyclodextrins on silicon oxide surfaces. Langmuir 20, 5460-5466 (2004).

39. J. Korn, M. \& S. Cramer, K. Windowing chicken eggs for developmental studies. Journal of Visual Experiments e306 (2007).

40. Blacher, S. Quantification of angiogenesis in the chicken chorioallantoic membrane (CAM). Image Analysis \& Stereology 24, 169-180 (2005). 


\title{
Chapter 6
}

\section{Insights in the surface dynamics of weakly multivalent systems}

\begin{abstract}
In this chapter fluorescent guest peptides were microcontact printed on surfaces coated with $\beta$-CD receptor sites. In the presence of competitive host molecules in the surrounding medium, competition-induced surface spreading of the guest peptides was studied. The position of the tyrosine $(\mathrm{Y})$ and phenylalanine $(\mathrm{F})$ moieties within the guest peptides was varied and the effect of valency, individual affinity, and positioning of the guest moieties on spreading dynamics was investigated. A pentavalent peptide containing five tyrosines $\left(\mathrm{Y}_{5}\right)$ showed a lower spreading rate at low $\beta$-CD concentrations when compared to hexavalent peptides of the same overall affinity that contained also $\mathrm{F}$ sites $\left(\mathrm{FY}_{4} \mathrm{~F}\right.$ and $\left.\mathrm{YF}_{4} \mathrm{Y}\right)$. $\mathrm{Y}_{5}$ followed a sudden rise in spreading rate at intermediate $\beta$-CD concentrations with concomitant desorption of the peptide from the surface, suggesting a transition in spreading mechanism. Although the hexavalent $\mathrm{FY}_{4} \mathrm{~F}$ and $\mathrm{YF}_{4} \mathrm{Y}$ spread faster at low $\beta$-CD concentrations, desorption of these hexavalent peptides started to occur only at higher $\beta$-CD concentrations $\left(\mathrm{FY}_{4} \mathrm{~F}\right)$ or not at all $\left(\mathrm{YF}_{4} \mathrm{Y}\right)$. The apparently lower sensitivity towards competition from $\beta$-CD in solution is attributed to the lower intrinsic affinity of the F moiety for $\beta$-CD.
\end{abstract}




\subsection{Introduction}

Multivalent interactions play a key role in various biological transport mechanisms., ${ }^{1,2}$ Diffusion of multivalent biomolecules dictates the working of bio-signaling mechanisms at all length scales ranging from the molecular level, such as the bipedal motor protein diffusion along microtubules, to the cellular level, such as the movement of cells along blood capillaries. ${ }^{3,4}$ Remarkably in living cells, the motion of molecules is rarely three-dimensional but often shows two-dimensional (interfacial) or onedimensional (through pores) diffusion with an exquisite degree of control over directionality. For example, nuclear pores, which act as a gateway between the nucleus and the cytoplasm, exhibit one-dimensional diffusion of molecules with a high selectivity in transport provided by receptors along the interface., ${ }^{5,6}$ Another example involves the motion of adenosine triphosphate along actin filaments. Yet, due to the highly complex nature of the biological milieu, it is often very difficult to unravel the molecular and physicochemical aspects of such diffusion processes. Therefore, the field is in need of clear model systems taken out of the biological complexity, in order to understand these processes in more quantitative detail.

To study multivalent transport processes at an interface, a biological receptor system composed of phenylalanine glycine nucleoporins has been fabricated at a solid surface, and the diffusion of the complementary karyopherin receptors decorated on outside of colloidal particles was studied in the presence of various concentrations of karyopherins in the surrounding medium. ${ }^{5}$ It has been observed that the optimal binding affinity is crucial for diffusion of the colloidal particles along the surface comprising of phenylalanine glycine nucleoporins. The binding affinity was manipulated by adding different concentrations of free karyopherin receptors to the surrounding solution in order to effectuate the diffusion of the multivalent ligand along the surface.

To understand multivalent aspects of surface diffusion, a synthetic system has been reported by our group in which the diffusion of a multivalent adamantane ligand has been studied along a $\beta$-CD monolayer. ${ }^{7}$ Adamantane is known to form a strong hostguest complex with $\beta$-CD. In the case of bis-adamantane guest molecules, three different surface diffusion mechanisms, coined walking, hopping, and flying, have been 
observed, and the relative contributions of these mechanisms could be tuned by varying the concentration of competing $\beta$-CD in the solution. Recently it has been reported by Frenkel and coworkers that polymers that have their ligands clustered into contiguous domains, either located at the polymer chain ends or chain mid-section show differences in their surface adsorption behavior. ${ }^{8}$ This study used mean-field self-consistent field theory to show that when non-binding and strongly binding ligands are clustered into contiguous domains along the polymer chain, a higher selectivity in binding of the polymer to complementary binding surfaces can be observed when compared to cases where these ligands are uniformly distributed along the polymer chain.

In this chapter we show the design of a multivalent peptide system containing two types of binding moieties with different intrinsic binding affinities. We study the effects of valency, individual affinity, and positioning of aromatic amino acids in peptides on their diffusion dynamics at receptor surfaces. In that context, we designed three fluorescently labeled peptides containing different numbers of tyrosine $(\mathrm{Y})$ and phenylalanine $(\mathrm{F})$ moieties. Both these aromatic amino acids can form host-guest complexes with $\beta$-CD but with different individual affinities (K values of $120 \mathrm{M}^{-1}$ and $60 \mathrm{M}^{-1}$ for $\mathrm{Y}$ and $\mathrm{F}$, respectively). ${ }^{9}$ We synthesized three peptides, $\mathrm{Y}_{5}, \mathrm{FY}_{4} \mathrm{~F}$ and $\mathrm{YF}_{4} \mathrm{Y}$, that have a uniform distribution of sites, an equal spacing between the sites, and similar overall binding affinities. $\mathrm{Y}_{5}$ differs from the others in the valency, while $\mathrm{FY}_{4} \mathrm{~F}$ and $\mathrm{YF}_{4} \mathrm{Y}$ have a reversed positioning of the stronger $\mathrm{Y}$ and weaker $\mathrm{F}$ sites at the termini and core of the peptides. The fluorescent peptides were microcontact printed on $\beta$-CD surfaces, and changes of the printed patterns in time were monitored at different concentrations of $\beta$ CD in solution. The implemented methodology and mechanisms of multivalent spreading are explained in this chapter.

\subsection{Results and discussion}

\subsubsection{Spreading of multivalent peptides in printing experiments}

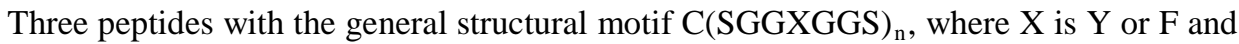
$\mathrm{n}$ is 5 or 6 , were synthesized using solid phase peptide synthesis. After purification of the crude peptides, the cysteine at the terminal position of the purified peptide was coupled to Oregon-488 (Oregon Green, OG) via the thiol-maleimide reaction. The 
purity of the dye-conjugated peptides was verified by analytical HPLC and ESI mass spectrometry as described in the Materials and methods section. The overall binding affinities with the $\beta$-CD monolayer were determined using surface plasmon resonance (SPR) spectroscopy for the parent peptides lacking the dye, and the log $K_{L M}$ values were found to be 5.49, 5.85 and 5.97 for $\mathrm{Y}_{5}, \mathrm{YF}_{4} \mathrm{Y}$ and $\mathrm{FY}_{4} \mathrm{~F}$, respectively. These values agree with the programmable affinity model described in Chapters 2 and 3, and the results indicate that the overall affinities of these peptides with the $\beta$-CD surface are quite similar.

a

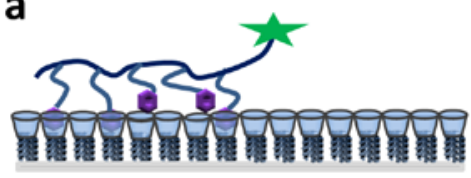

Adsorbed peptide

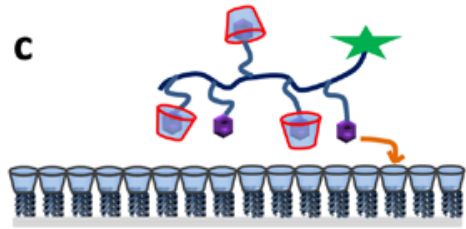

Hopping

e

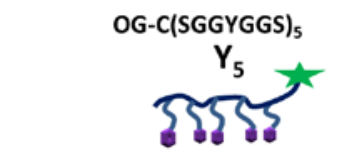

OG-CSGGYGGS(SGGFGGS) 4 SGGYGGS

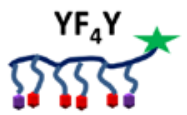

OG-CSGGFGGS(SGGYGGS) ${ }_{4}$ SGGFGGS

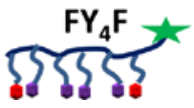

b

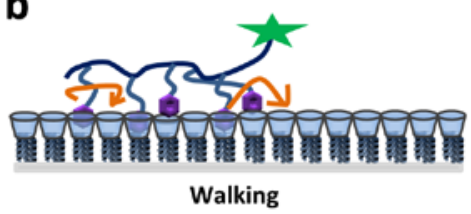

d

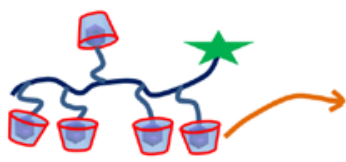

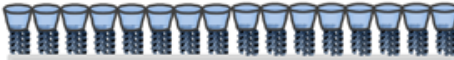

Flying

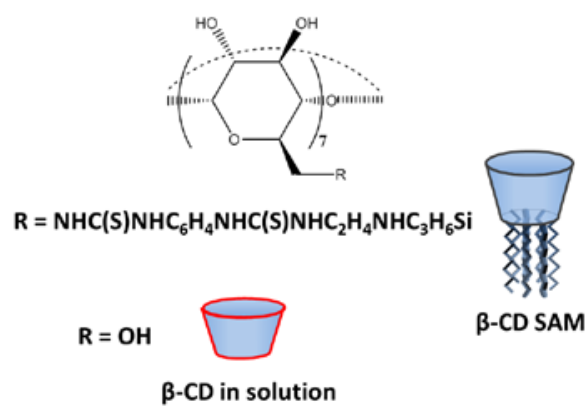

Figure 6.1 - Multivalent surface diffusion: a-d) Cartoons showing basic mechanisms involved in multivalent surface diffusion. e) Supramolecular components used in the model system. 
$\beta$-CD monolayers on glass surfaces were fabricated using a protocol described before, and the multivalent peptides were assembled onto these monolayers by microcontact printing ( $\mu \mathrm{CP}$ ) (Figure 6.2). ${ }^{7,}{ }^{10}$ Solutions of $20 \mu \mathrm{M}$ of peptide were used to print lines of $5 \mu \mathrm{m}$ wide with a spacing of $25 \mu \mathrm{m}$ in all printing experiments.

a

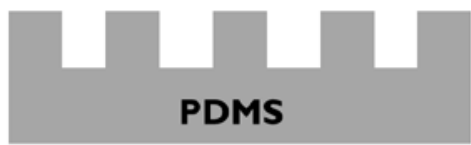

Inking (2 min)
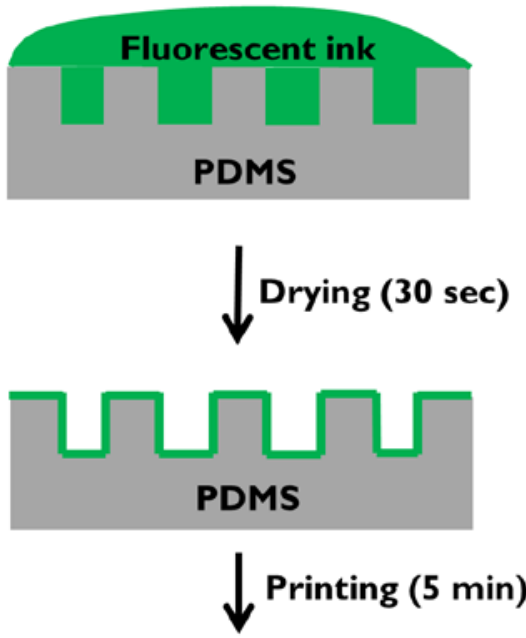

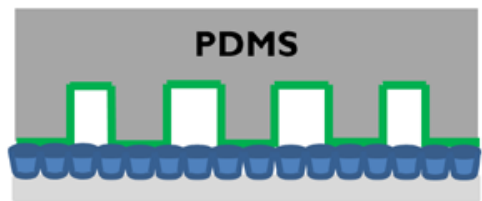

$\downarrow^{\text {stamp lift off }}$

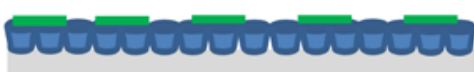

b

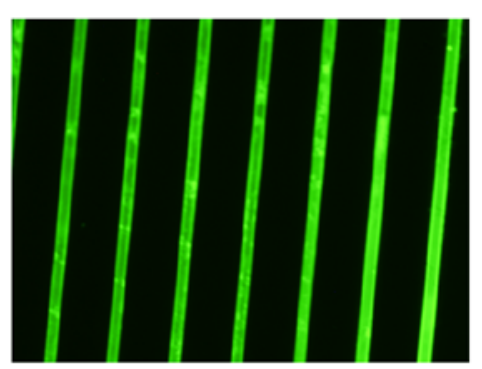

Figure 6.2 - a) Schematic representation of the microcontact printing process employed for immobilization of the multivalent peptides on the $\beta$-CD surface. b) A representative fluorescence microscopy image of $\beta$-CD surface after printing of the $\mathrm{Y}_{5}$ peptide.

After printing, the sample was covered with a rubber seal with a diameter of $2 \mathrm{~cm}$ leaving an accessible surface area of $2.86 \mathrm{~cm}^{2}$, and $500 \mu \mathrm{l}$ of solution was added on top of the sample. Peptides printed on the $\beta$-CD surface and held by host-guest interactions did not spread in dry conditions (Figure 6.1a). However, in the presence of water or $\beta$ $\mathrm{CD}$ solution, the guest molecules diffused on the host surface. The addition of $\beta$-CD 
molecules (in solution), which act as a mobile host, induced the competition for the aromatic amino acid guest sites in the peptides with the host sites at surface. Thus each peptide was investigated for their spreading behavior in the absence and presence of varying concentrations (0-6 mM) of $\beta-C D$ in solution.

The evolution of the supramolecular patterns in time was monitored by fluorescence microscopy. As a typical example, when a printed pattern of $\mathrm{Y}_{5}$ was incubated with a 2 $\mathrm{mM} \beta$-CD solution, the fluorescent lines started to "fade" within minutes (Figure 6.3a). The fluorescent lines were converted to intensity profiles as described before. ${ }^{7}$ In a typical fluorescence intensity profile as shown in Figure 6.3b, the intensity difference $(\Delta I)$ quantitatively represents the initial surface coverage of peptides printed on the $\beta$ CD surface. $I_{\max }, I_{\min }$ and $w$ were used to describe the changes in the supramolecular pattern in time. In particular, the change of the peak width $w$ with time (Figure 6.4c) was fitted to a line and the slope was used as the spreading rate.

a

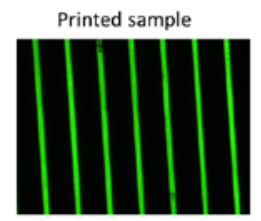

b

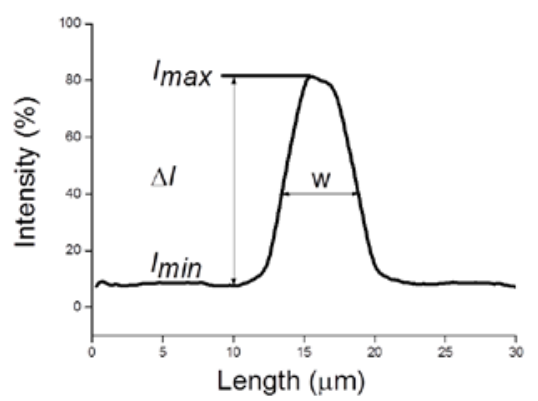

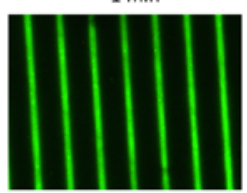

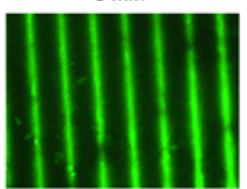

C
$5 \mathrm{~min}$

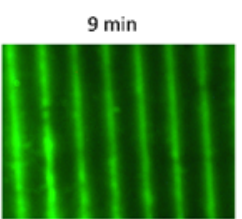

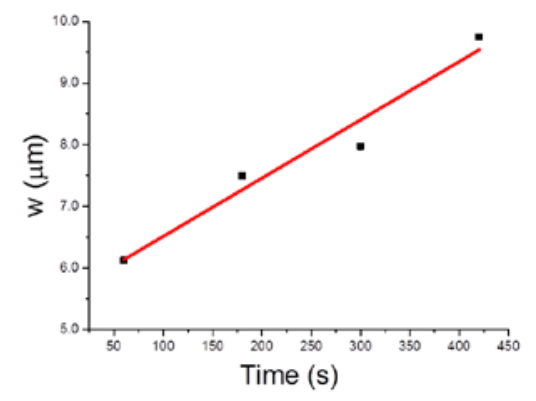

Figure 6.3 - a) Evolution of surface patterns of $\mathrm{Y}_{5}$ peptide as a function of time microcontact printed from a $20 \mu \mathrm{M}$ solution, rinsed and dried, and subsequently immersed (at $\mathrm{t}=0$ ) in a $2 \mathrm{mM}$ $\beta$-CD solution. b) Intensity profile as measured by integrating intensities over a section of a fluorescence microscopy image (printed sample) perpendicular to the lines, with parameters $w$ (full peak width at half maximum) and $\Delta I$ (intensity difference at peak maximum). c) Width (w) increase as a function of time; the slope of a line fitted to the data represents the spreading rate. 
For each peptide the spreading rates were calculated at varying $\beta$-CD concentrations. Figure 6.4 shows the resulting dependence of the spreading rate on the $\beta$-CD competitor concentration, for the peptides $\mathrm{Y}_{5}, \mathrm{YF}_{4} \mathrm{Y}$ and $\mathrm{FY}_{4} \mathrm{Y}$.

The spreading rate of $Y_{5}$ did not increase monotonously with the $\beta$-CD concentration. At low concentrations $(0-1 \mathrm{mM})$ of $\beta$-CD in solution, the spreading rate was low. Between 1 - $2 \mathrm{mM}$ a sudden increase in spreading rate was observed, while from 2 to 6 $\mathrm{mM}$ no further significant change in spreading rate was observed. At $\beta$-CD concentrations of $2 \mathrm{mM}$ and higher, the intensities (area under the intensity profiles as shown in Figure 6.3) decreased notably, while this effect was absent at low concentrations. This indicates desorption co-occurring with spreading at these higher concentrations. The decrease in $I_{\max }$ in this range is thus caused by both spreading on the surface and desorption from the surface into the bulk solution. As can be observed in Figure 6.4, the desorption is also signified by the rapid increase of the spreading rate at $2 \mathrm{mM} \beta-\mathrm{CD}$ and up.

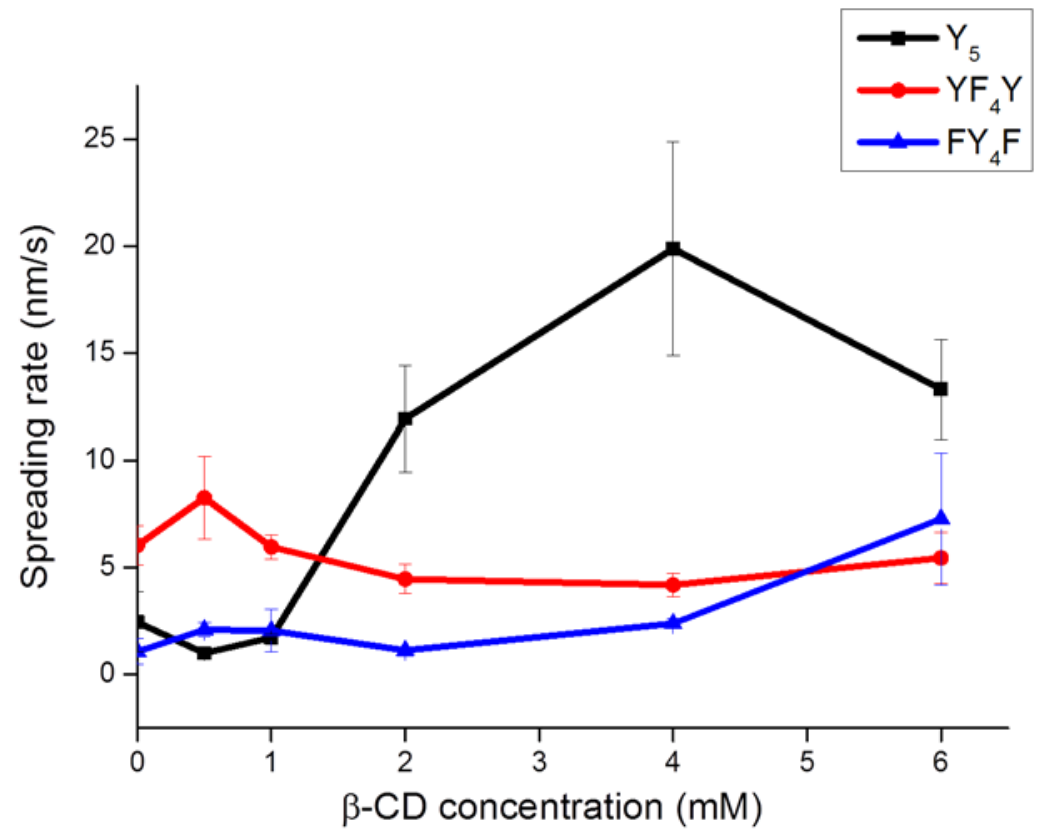

Figure 6.4 - Calculated spreading rates as a function of the $\beta$-CD concentration in solution for printed samples of $\mathrm{Y}_{5}, \mathrm{YF}_{4} \mathrm{Y}$ and $\mathrm{FY}_{4} \mathrm{~F}$. (Error bars represent single standard deviations for two or more independently performed measurements with at least three printed lines per sample for each $\beta$-CD concentration) 
The hexavalent $\mathrm{FY}_{4} \mathrm{~F}$ peptide showed similar spreading rates at low $\beta$-CD concentrations as $Y_{5}$. Interestingly, the rates did not change significantly up to $4 \mathrm{mM} \beta$ CD. Only at $6 \mathrm{mM} \beta$-CD, desorption of the peptide from the surface was observed. The $\mathrm{YF}_{4} \mathrm{Y}$ peptide, which has a core composed of the weaker binding $\mathrm{F}$ and termini of the stronger binding $\mathrm{Y}$, showed higher spreading rates than both other peptides at low $\beta$-CD competitor concentrations. Notably, across the whole range of $\beta$-CD concentrations tested here, neither desorption was visible for this peptide, nor did the spreading rate change appreciably. The slightly increased spreading rate at $0.5 \mathrm{mM} \beta$-CD compared to the rates at $0 \mathrm{mM}$ and $1 \mathrm{mM} \beta$-CD can possibly be attributed to a change of diffusion mechanism from mainly walking to hopping, in analogy to the increase in spreading rate observed for a divalent adamantyl derivative. ${ }^{7}$ Yet, this maximum in spreading rate is much less pronounced for the peptide studied here, as witnessed by the approx. 1.3fold increase in spreading rate obtained at $0.5 \mathrm{mM}$ vs that at $0 \mathrm{mM} \beta-\mathrm{CD}$, in comparison with the $>10$-fold enhancement in the case of the divalent adamantyl derivative. ${ }^{7}$

Collectively, the $\mathrm{Y}_{5}$ and $\mathrm{FY}_{4} \mathrm{~F}$ peptides showed lower spreading rates in the absence of desorption compared to $\mathrm{YF}_{4} \mathrm{Y}$. This is tentatively attributed to the presence of larger numbers of the stronger binding $\mathrm{Y}$ in the former two peptides and/or the positioning of the $\mathrm{Y}$ moieties in the core of these peptides. The early onset of desorption for $\mathrm{Y}_{5}$ at 2 $\mathrm{mM} \beta$-CD, compared to $\mathrm{FY}_{4} \mathrm{~F}$ and $\mathrm{YF}_{4} \mathrm{Y}$, can possibly be attributed to the difference in valency. However, another factor that is likely involved here is the fact that the weaker binding $\mathrm{F}$ also interacts less with $\beta$-CD in solution, and therefore is less sensitive to the increase of the concentration of this competitor. Thus it can be expected that peptides with larger numbers of the weaker binding $\mathrm{F}$ show less dependence on the $\beta$-CD concentration and exhibit an onset of desorption only at higher concentrations.

Also important to note is that all spreading rates, obtained in the absence of desorption, were higher for all three peptides compared to the divalent adamantyl derivative studied before. In the case of $\mathrm{YF}_{4} \mathrm{Y}$, the difference was even more than an order of magnitude. For the adamantyl derivatives, only the divalent guest showed appreciable spreading while the trivalent one was already apparently complexed irreversibly. In sharp contrast, the peptides with valencies of 5 and 6 were more mobile than the divalent adamantyl derivative. Equally important, this high mobility of the weaker binding peptides is paired to an equal $\left(\mathrm{Y}_{5}\right.$; both show an onset of desorption at $2 \mathrm{mM} \beta$-CD) or lesser 
$\left(\mathrm{FY}_{4} \mathrm{~F}\right.$ and $\left.\mathrm{YF}_{4} \mathrm{Y}\right)$ sensitivity for desorption. Again, this is attributed in part to the larger overall valencies of the peptides as well as the lesser sensitivity of the amino acid guest moieties to competition.

\subsubsection{Fluorescence recovery after photobleaching (FRAP) experiments}

In the above mentioned printing strategy, the gradient of effective concentration $C_{\text {eff, }}$, which is the concentration of free $\beta$-CD cavities at the surface, is known to play a role in the spreading of multivalent guests along the $\beta$-CD surface. ${ }^{7}$ In the case of the adamantyl guests studied before, these guests typically use all available legs to bind to the surface, and very low fractions of free $\beta$-CD host units can be reached before the partial release of binding sites is thermodynamically promoted. This creates a large difference between the fractions of free $\beta$-CD cavities in the printed and empty surface areas directly after the printing step. This was witnessed by a large difference in spreading rate between printed lines and samples that were subsequently backfilled with another guest, with an approx. 5-fold faster spreading in the former case. ${ }^{7}$ In contrast, the peptides used here do not bind with all of their legs to the $\beta$-CD surface simultaneously, even at low coverages, and therefore the contrast in $C_{\text {eff }}$ between the printed and empty areas is expected to be a lot lower. Therefore we measured the diffusion of peptides in the absence of a concentration gradient, by using fluorescence recovery after photobleaching (FRAP).

By FRAP, fluorescent molecules at the monolayer surface are photo-bleached and the recovery of fluorescence is monitored over a period of time. If the molecules are mobile, the fluorescence intensity of the bleached spot is recovered. Therefore, FRAP is commonly used to quantify the lateral diffusion of molecules along a surface. ${ }^{11}$ In applying FRAP to our peptide-covered $\beta$-CD surfaces, the photobleaching of the peptide-appended dye is expected not to influence the binding properties of the peptide. Therefore, when photobleaching a part of a $\beta$-CD surface that is homogeneously covered by peptide guests, the density of peptides is expected to remain unaltered as well as the fraction of free $\beta$-CD cavities. 

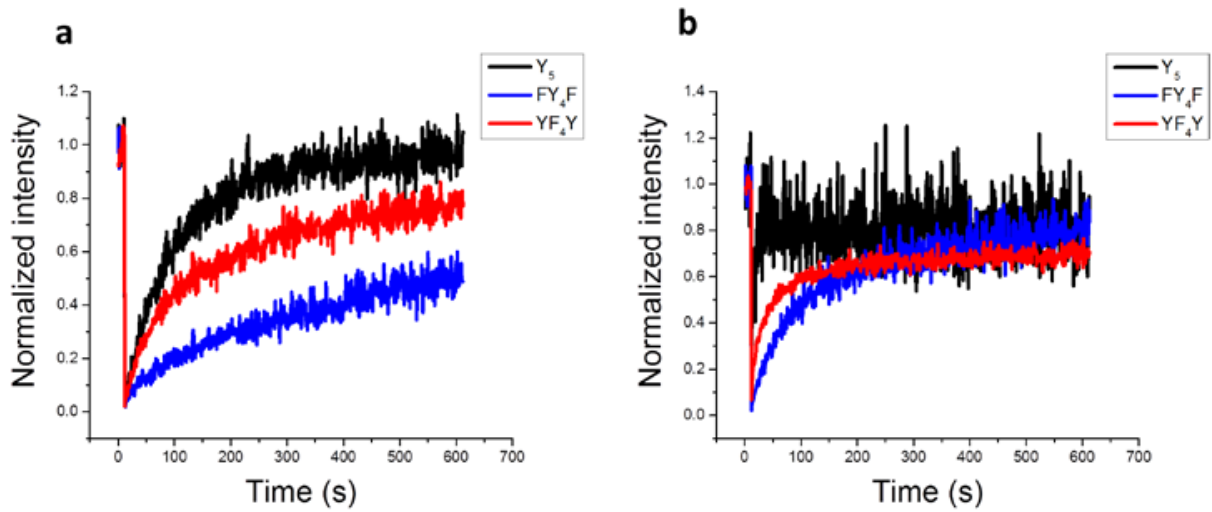

Figure 6.5 - FRAP recovery curves of peptides (present in a monolayer) under a) $0 \mathrm{mM}$ and b) 2 $\mathrm{mM} \beta$-CD solution.

\begin{tabular}{|c|c|c|c|c|}
\hline Peptide & {$[\beta-\mathrm{CD}]$} & $\begin{array}{c}\text { Spreading rate } \\
\text { (printing) } \\
\text { (nm/s) }\end{array}$ & $\begin{array}{c}D^{1} \\
\text { (FRAP) } \\
\left(\mu \mathrm{m}^{2} / \mathrm{s}\right)\end{array}$ & $\begin{array}{c}\text { Spreading rate } \\
\text { (FRAP) } \\
\text { (nm/s) }\end{array}$ \\
\hline $\mathrm{Y}_{5}$ & $0 \mathrm{mM}$ & $2.5 \pm 1.4$ & $0.08 \pm 5 \mathrm{E}-5$ & $3.6 \pm 0.001$ \\
\hline $\mathrm{Y}_{5}$ & $2 \mathrm{mM}$ & $11.9 \pm 2.4$ & $1.2 \pm 0.02$ & $13.9 \pm 0.08$ \\
\hline $\mathrm{YF}_{4} \mathrm{Y}$ & $0 \mathrm{mM}$ & $6.0 \pm 0.9$ & $0.04 \pm 0.01$ & $2.7 \pm 0.3$ \\
\hline $\mathrm{YF}_{4} \mathrm{Y}$ & $2 \mathrm{mM}$ & $4.5 \pm 0.7$ & $0.11 \pm 0.03$ & $4.2 \pm 0.8$ \\
\hline $\mathrm{FY}_{4} \mathrm{~F}$ & $0 \mathrm{mM}$ & $1.1 \pm 0.9$ & $0.013 \pm 0.006$ & $1.5 \pm 0.3$ \\
\hline $\mathrm{FY}_{4} \mathrm{~F}$ & $2 \mathrm{mM}$ & $1.1 \pm 0.7$ & $0.04 \pm 0.02$ & $2.5 \pm 0.6$ \\
\hline
\end{tabular}

${ }^{1}$ In the case of FRAP experiments, the diffusion coefficient $(D)$ was calculated from fitting of the FRAP recovery curves with a 1D diffusion model. ${ }^{2}$ The diffusion coefficients were converted into spreading rates $(\mathrm{nm} / \mathrm{s})$ by simulating a spreading experiment using the $D$ values between 10 $40 \mathrm{~min}$, giving the formula: spreading rate $(\mathrm{nm} / \mathrm{s})=12.6 \times \sqrt{\mathrm{D}}\left(\mathrm{um}^{2} / \mathrm{s}\right)$.

Table 6.1 - Spreading rates of multivalent peptides determined from printing and FRAP experiments. Error values $(1 \sigma)$ were deduced from two or more independent experiments.

The FRAP recovery profiles were recorded either in the presence of water or a $2 \mathrm{mM} \beta$ CD solution (Figure 6.5) and were fitted to a 1D diffusion model to calculate the diffusion coefficients for each condition. ${ }^{12}$ In principle, multivalent diffusion is not 
identical to Fick's diffusion as in the former case the binding affinity is related to the fraction of free surface sites in a more complex manner. ${ }^{7}$ However, the FRAP data showed a good fit with the Fick's diffusion model, likely because the fraction of free surface sites remained constant in this experiment. Thus we used the apparent diffusion coefficients obtained from the fitting of the FRAP data to predict the spreading rates of the peptide, as shown in Table 6.1.

The spreading rates obtained from the FRAP measurements were in good agreement with the ones obtained from the printing experiments, only the values for the peptide $\mathrm{YF}_{4} \mathrm{Y}$ at $0 \mathrm{mM} \beta$-CD concentration deviated from each other. The generally good agreement between the printing and FRAP experiments indicates that the influence of a change in effective concentration is marginal for the peptide systems. As indicated above, we attribute the negligible dependence on effective concentration in the case of the peptides to the partial use of the guest moieties in binding and in the resulting higher fractions of free $\beta$-CD sites at the surface. This is in contrast to the divalent adamantyl guest, which showed much faster diffusion when printed.

\subsubsection{Theoretical considerations}

\subsubsection{Thermodynamics}

In order to understand the differences observed in peptide diffusion, we aimed to simulate the speciation of all species present at the $\beta$-CD surface at varying competitor concentrations. At the same time, we aimed to compare the spreading data with the reported bis-adamantane (bis-Ad) system. ${ }^{7}$

Initially, we compared the affinities of the guest moieties (Y, F, Ad) and their abilities to form intramolecular bonds at the surface. We assumed the molecules (peptide or divalent adamantane ligand) to bind at an infinitely dilute concentration on the surface, in which case the effective molarity $(E M)$ is equal to $E M_{\max }$. The binding constant $K_{\text {intra }}$, which describes the binding of a guest moiety in a multivalent molecule that is already bound to the $\beta$-CD surface by other interaction sites, is given by $K_{i} \times E M$, where $K_{i}$ is the intrinsic affinity of the guest moiety. We call this factor $K_{i} \times E M$ the multivalent enhancement factor because the overall affinity of an n-valent guest binding with the maximal valency is given by $K_{o v}=K_{i} \times\left(K_{i} \times E M\right)^{n-1} . K_{\text {intra }}\left(=K_{i} \times E M\right)$ is equal to the ratio of bound and unbound guest sites. ${ }^{13}$ Therefore, the fraction of bound guest 
moieties can be estimated by $K_{i} \times E M /\left(1+K_{i} \times E M\right)$. Table 6.2 gives the $K_{i}$ and $E M$ values, as well as the bound and the unbound fractions derived from these, calculated in the absence of competitor. The values for the bound and unbound fractions show that, at any given moment in time, a sizeable fraction of the peptide guest moieties remains unbound, approx. 16\% in the case of Y and even $28 \%$ in the case of F.

\section{Tyrosine or Phenylalanine}

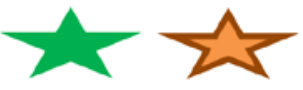

Fluorescent groups

\section{Adamantane}
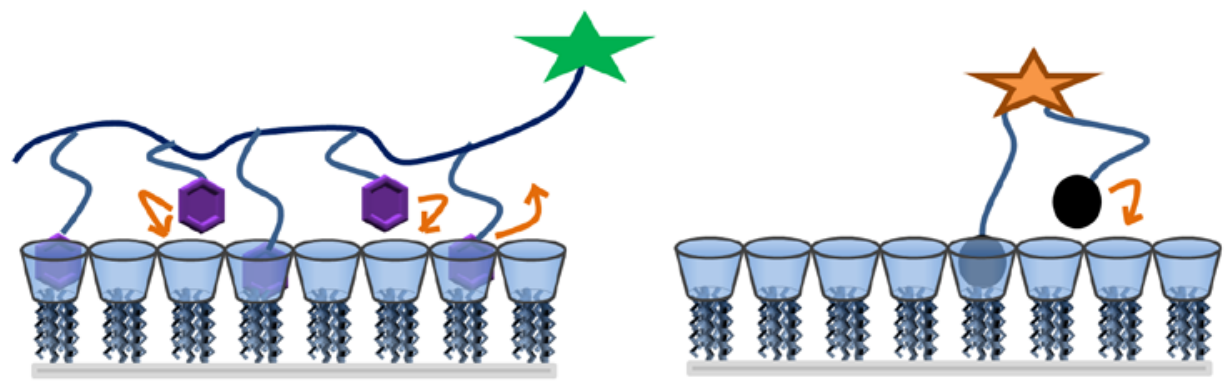

Figure 6.6 - Cartoon showing the equilibrium of individual guest sites when binding to the $\beta$-CD surface in case of the designed peptide (left) or adamantane (right) systems.

\begin{tabular}{|c|c|c|c|c|}
\hline Guest & $K_{i}\left(\mathrm{M}^{-1}\right)$ & $E M(\mathrm{M})$ & Bound (\%) & Unbound (\%) \\
\hline F & 60 & 0.043 & 72 & 28 \\
\hline $\mathrm{Y}$ & 120 & 0.043 & 84 & 16 \\
\hline $\mathrm{Ad}$ & 50000 & 0.1 & 99.98 & 0.02 \\
\hline
\end{tabular}

Table 6.2 - Thermodynamic parameters ( $K_{i}$ and EM) and the fractions of bound and unbound species for surface-bound guest moieties as calculated from these parameters, at infinitely dilute concentration of multivalent guest. $K_{i}$ of $\mathrm{Y} / \mathrm{F} / \mathrm{Ad}$ is reported in literature ${ }^{9,13}$ while $E M$ for $\mathrm{Y} / \mathrm{F}$ is extracted from modelling data discussed in Chapter 3 and for adamantane as reported previously. ${ }^{13}$ 
In the case of bis-Ad, only $0.02 \%$ of the sites are unbound. Obviously, there is a dynamic exchange between the bound and unbound states, at a rate that is primarily governed by the spontaneous dissociation rates $\left[\mathrm{s}^{-1}\right]$ of the bound guest moieties, which scales inversely with the affinity $K_{i}$. We attribute the experimentally observed difference in spreading rate between the divalent adamantane guest (a very low spreading rate (close to zero) at $0 \mathrm{mM} \beta$-CD concentration) in our previous study compared to the peptides in the current study to the much smaller fractions of unbound sites for the former. Similarly, peptide guests containing a majority of tyrosine groups $\left(\mathrm{Y}_{5}, \mathrm{FY}_{4} \mathrm{~F}\right)$ showed lower spreading rates than $\mathrm{YF}_{4} \mathrm{Y}$ which showed the highest spreading rate of all which can then be ascribed to the lowest number of simultaneously bound ligand moieties for this peptide. At zero or low competitor concentrations, the walking mechanism, which is the lateral surface diffusion of multivalent molecules for species in which at least one site remains bound during motion (Figure 6.1b), is expected to be dominant, and the rate of it will depend on the population of both the bound and unbound states and on the exchange rate between them. Basically, the weakening of the intrinsic affinity upon going from Ad to $\mathrm{Y}$ to $\mathrm{F}$ causes a more balanced population of both the bound and unbound states as well as a concomitant, faster dissociation rate of the bound state.

Another important observation has been the difference in onsets of visible desorption as a function of competitor concentration: for the bis-Ad guest, desorption occurred at about $2 \mathrm{mM} \beta$-CD, which was similar for $\mathrm{Y}_{5}$, but only at higher $\beta$-CD concentrations (6 $\mathrm{mM}$ ) for $\mathrm{FY}_{4} \mathrm{~F}$, and even higher (not observed in the currently used range) for $\mathrm{YF}_{4} \mathrm{Y}$. To explain the differences in spreading in the presence of competing $\beta-C D$, it is noted that the binding of a guest moiety that is not bound to a surface site, with $\beta$-CD from solution is governed by: $K=K_{i} \times[\beta-\mathrm{CD}]$. This explains in general terms that guests with a higher $K_{i}$ are more sensitive to competition. Complicating the direct comparison between the bis-Ad derivative and the peptides are the difference in valency and the difference in population of the bound and unbound states as explained above. Therefore, both the higher dependence on competition and the lower valency of the bis-Ad guest cause the observed early onset of desorption, while the higher efficiency in intramolecular bond formation, and the resulting higher overall affinity, is counteracting these effects. 
Yet there is another important factor known as "rebinding" which is known to play a role in the binding kinetics and desorption of proteins and particles to surfaces. ${ }^{14}$ According to this concept, when a ligand adsorbed on a surface desorbs into the solution, it has a probability of quickly rebinding to the surface depending on the concentration of free sites on the surface to readsorb to and on the diffusion rate of the molecule into the bulk. Qualitatively, rebinding is high when the desorption is limited by mass transfer (slow diffusion into solution compared to readsorption) and low when dissociation from the binding site is rate-limiting. A more quantitative description has been worked out for monovalently binding ligands to surface-bound receptor sites. ${ }^{14}$ Consider a reversible reaction between a ligand with a receptor surface with simultaneous diffusion into solution such that:

$$
\mathrm{A}+\mathrm{B} \underset{k_{d}}{\rightleftharpoons} \mathrm{k}
$$

Where $A$ is the concentration of the ligand in solution which is in equilibrium with a density of molecules on the surface, $C$, and $B$ is the surface coverage of sites on the surface which are not occupied by A. $k_{a}$ and $k_{d}$ are the association and dissociation rate constants of A with the receptor surface. In this case parameter $b$, which is a measure of the probability of rebinding of a ligand to the surface is given by:

$$
\begin{aligned}
& b=\frac{C}{A} \frac{1}{\gamma} ; \\
& \frac{C}{A}=\frac{N}{K_{d}+A}
\end{aligned}
$$

Where $\gamma$ is the average distance travelled by molecule in solution during the average surface residence time $k_{d}^{-1}$. N is the total density of surface sites (occupied and unoccupied), and $K_{d}$ is the dissociation equilibrium constant $\left(=k_{d} / k_{a}\right)$. The value of $N$ in case of the $\beta$-CD monolayer is reported in literature to be $6 \times 10^{-11} \mathrm{~mol} / \mathrm{cm}^{2}{ }^{13}$ The fraction $C / A$ is the thickness of a slab in solution that contains molecules equal to the density of molecules adsorbed on the surface. $\gamma$ can be expressed as 


$$
\gamma=\sqrt{\frac{D}{k_{d}}}
$$

Where $D$ is the diffusion coefficient of the ligand in solution. If $\gamma \gg C / A$, the molecule will diffuse away from the surface and rebinding is not favored whereas when $\gamma \ll C / A$, diffusion into solution will be comparatively slower, and rebinding is favored. Thus we tried to calculate the $C / A$ and $\gamma$ values for the bis-Ad and hexavalent $\left(\mathrm{FY}_{4} \mathrm{~F}\right)$ peptide system. The divalent adamantane has an overall affinity of: $K_{a}=K_{i}^{2} \times E M=2.5 \times 10^{8}$ $\mathrm{M}^{-1}$ thus $K_{d}=4 \mathrm{nM}$. For the $\mathrm{FY}_{4} \mathrm{~F}$ peptide, taken as a representative for all peptides: $K_{a}$ $=K_{i}\left(K_{i} \times E M\right)^{5}=9.33 \times 10^{5} \mathrm{M}^{-1}$ thus $K_{d}=1 \mu \mathrm{M}$. At initial conditions where all the ligands are bound to the surface, i.e. $\mathrm{A}=0: C / A=N / K_{d}$ (equation 3). Thus for the bisAd guest, $C / A=1.25 \times 10^{5} \mu \mathrm{m}$ and for $\mathrm{FY}_{4} \mathrm{~F}, \mathrm{C} / \mathrm{A}=600 \mu \mathrm{m}$. Similarly for the bis-Ad guest, $\gamma=15 \mu \mathrm{m}$, while that for $\mathrm{FY}_{4} \mathrm{~F}$ is $0.1 \mu \mathrm{m}$ (diffusion coefficients for both systems are assumed to be the same, i.e. $D=0.013 \mu \mathrm{m}^{2} / \mathrm{s}$ ).

In both cases, it follows that $\gamma \ll C / A$, which means that rebinding is highly favored. For the adamantane system, the value of the rebinding parameter $b$ was found to be $8.3 \times$ $10^{3}$ while for the hexavalent peptide $\left(\mathrm{FY}_{4} \mathrm{~F}\right), b=600$. Using the equation (3) for $\mathrm{C} / \mathrm{A}$ given above, we can calculate the value of $A$, i.e., the amount of ligand in solution, that is sufficient to inhibit the rebinding of ligands, which occurs when $b$ drops below 1 . In the case of the bis-Ad system, a micromolar concentration of ligand is already sufficient to lower $b$ to values below 1 . Due to the small dissociation constant of the bis-Ad system, molecules already present in solution can promptly bind to unoccupied sites on the $\beta$-CD surface before freshly desorbed molecules do. In the case of the peptides, however, a millimolar concentration of ligand is required to decrease the value of $b$ to below 1 . This means that, up to this peptide concentration, rebinding of newly desorbed peptides will be favorable over the binding of ones already present in solution. There is most likely an additional effect from the peptide keeping more of the surface $\beta$-CD sites free than the bis-Ad guest does, so that the number of available uncomplexed surface sites $(B)$ remains high for the peptides, while it is generally a lot lower for the bis-Ad derivative. Overall, these calculations confirm that also the weakly binding peptides can have large extents of rebinding, causing little or no desorption at low competitor concentrations. 
Equally important is to see what is the effect of the addition of competing $\beta$-CD on rebinding. The complexation with $\beta$-CD from solution leads to a decrease of the fraction of free, unbound guest sites also for guests that have completely desorbed from solution. At the same time, competition lowers the apparent affinity of a guest for the surface. In the equation (3) for $C / A$, this means that, upon increasing the competitor concentration, $A$ is decreased while $K_{d}$ is increased. So, even though desorption leads to an increase of the overall guest concentration, the concentration of guests that can re-adsorb, because they have free guest sites to form the interaction pairs, is actually going down. Therefore, the main effect on $b$ is the lowering of $C / A$ caused by the increase of $K_{d}$. As discussed above, both the lower valency and the higher efficiency in binding guest moieties with $\beta$-CD from solution in the case of the bis-Ad guest in comparison to the peptides, are causing that $K_{d}$ is increasing faster for the Ad system. This explains the early (at low $[\beta \mathrm{CD}]$ ) onset of desorption for the Ad system because rebinding gets unfavorable already at these relatively low competitor concentrations.

All considerations given so far above ignore the multivalent nature of the binding of the guests to the surface. To gain further understanding into the multivalent parameters involved in spreading of peptides on the $\beta$-CD surface, a thermodynamic equilibrium model was developed. The model is based on a full, explicit description of all the species involved in spreading using the intrinsic binding affinities $\left(K_{i}\right)$ of tyrosine and phenylalanine and the effective molarity $(E M)$ as the main model parameters. The model is an extension of the multivalent surface binding model developed before. ${ }^{13}$ There is, however, one main difference: the peptide guests implemented here are treated as linear systems, thus allowing the monitoring of the binding mode of each guest moiety separately as shown in Figure 6.7.

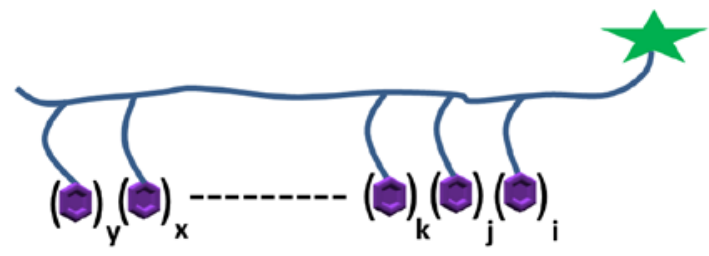

Figure 6.7 - Cartoon showing the linear peptide with arbitrary indication of sites. 
Every intermolecular surface binding of the first site i interacting with the surface is calculated from $K_{i}$ of the $\mathrm{i}^{\text {th }}$ site. All other species with higher valencies are calculated from the monovalent species for which site $\mathrm{i}$ is the one with the lowest number. This approach allows the introduction of a dependence of $E M$ on the spacing between the guest sites. An inverse relationship between $E M$ and the spacing is assumed here. In other words, the model assumes $E M_{i j}=E M_{0} /(j-i)$, where $j>i$, for species for which no sites are bound to the surface between sites $i$ and $j . E M_{0}$ is equal to $E M_{\max }(43 \mathrm{mM})$ at low coverage, but scales with the fraction of free surface $\beta-\mathrm{CD}$ sites $\theta_{\mathrm{f}}$, so $E M_{i j}=$ $\left(E M_{\max } \times \theta_{\mathrm{f}}\right) /(j-i)$. Using this model, the species distribution as a function of peptide concentration and of competitor concentration can be calculated.

For an arbitrary hexavalent peptide each guest moiety can be either: bound to a surface $\beta$-CD site, bound to a $\beta$-CD from solution, or remain unbound. Therefore, one can expect up to $3^{6}=729$ different species, of which $2^{6}(=64)$ are solution species and the remaining 665 are surface species. These are hugely enhanced numbers compared to the bis-Ad system, for which there exist 3 solution and 3 surface species. For clarity, it is therefore mandatory to make intelligible collections of species concentrations, for example, by grouping the species with the same numbers of sites bound (valency of bound species) to the surface as shown in Figure 6.8 for the binding of increasing concentrations of peptides, in the absence of $\beta$-CD competitor. 

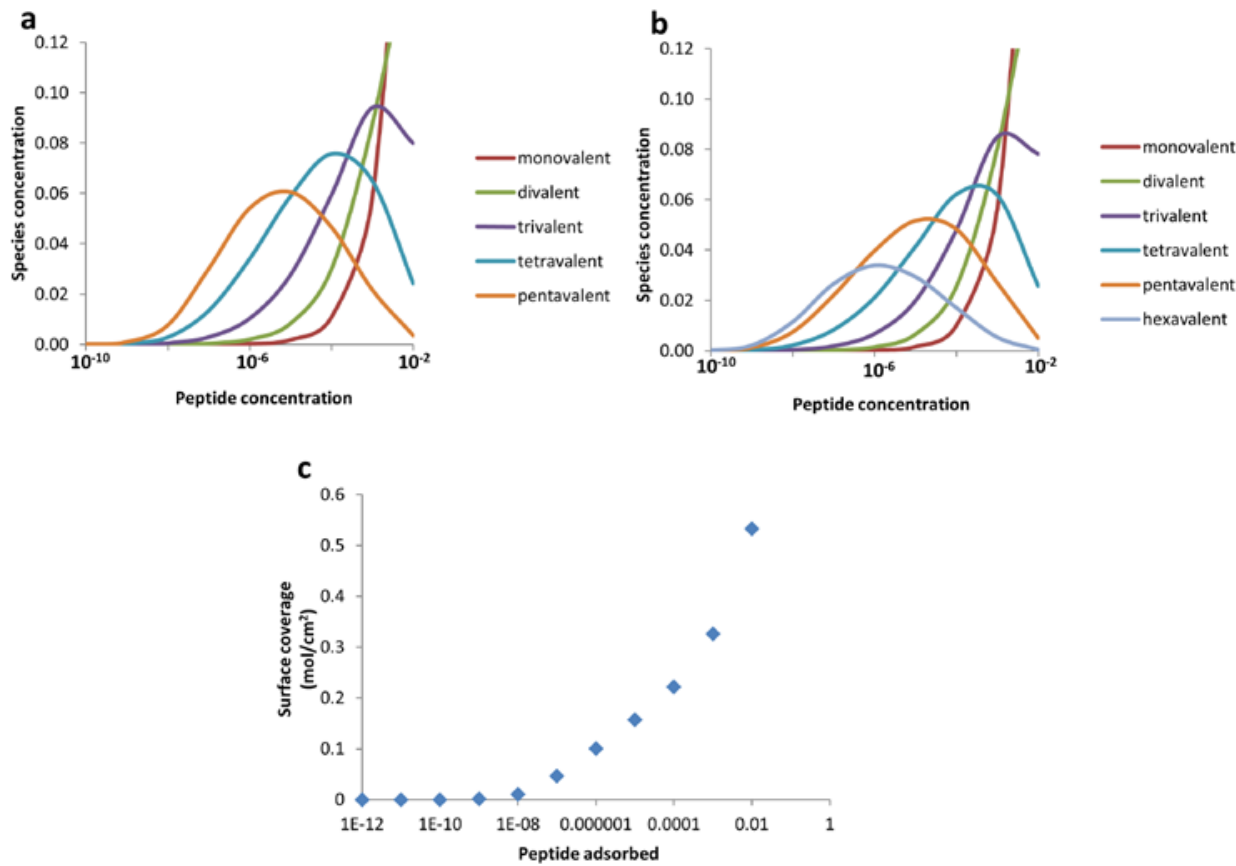

Figure 6.8 - Species distributions of a) pentavalent $\left(\mathrm{Y}_{5}\right)$ and $\left.\mathrm{b}\right)$ hexavalent $\left(\mathrm{FY}_{4} \mathrm{~F}\right)$ peptide at 0 $\mathrm{mM} \beta$-CD concentration. c) Graph of change in surface coverage as a function of total peptide concentration in solution.

The speciation in Figure 6.8 show several interesting aspects. First of all, already at the onset of adsorption at sub- $\mu \mathrm{M}$ concentrations, not only the species with all sites attached to the surface is observed, but also species with lower valencies are found. Upon increase of the peptide concentration in solution, a gradual decrease of the average valency of interaction is observed, as witnessed by the decrease of highervalent species and the increase of lower-valent ones. This is caused by the decrease of the fraction of free surface $\beta$-CD sites with increasing coverage, causing the decrease of $E M$ as explained above. ${ }^{13}$ Do note that this behavior is non-Langmuirian and causes the increase of coverage over a larger concentration range than for monovalent guests (Figure 6.7c). It is also distinctly different from the binding of strongly multivalent systems (with high $K_{i} \times E M$ factors, such as the bis-Ad system) where basically the fully bound (divalent) species is the only surface species present over a large concentration range (and for which the high-concentration regime at which valency is expected to go down never has been observed experimentally). 
Using this model we also calculated the decrease in the valency of the interaction of the multivalent peptides as a function of $\beta$-CD concentration as shown in Figure 6.9. This figure shows that the average binding valencies of the hexavalent peptides are over the whole range larger than those of the pentavalent $Y_{5}$. The valency of $Y_{5}$ with which it is bound to the surface at $2 \mathrm{mM} \beta$-CD is comparable to the valency of $\mathrm{YF}_{4} \mathrm{Y}$ at $9 \mathrm{mM} \beta$ CD. This underlines the difference in the onset of desorption between these peptides as discussed before. Also indicative may be the fraction of free sites at the solution species, as this determines the degree of rebinding: for example, the hexavalent $\mathrm{YF}_{4} \mathrm{Y}$ still has 4 (of 6) sites unbound at $10 \mathrm{mM} \beta$-CD, while for $Y_{5}$, the number of free sites drops below 4 (from 5) at $2 \mathrm{mM} \beta$-CD. That factor $4-5$ difference for the $\beta$-CD concentrations at which the valency drops below 4 is roughly also seen in the $\beta$-CD competitor concentration at which desorption starts to occur. Therefore, the overall valency is the main determining factor for predicting desorption vs rebinding.

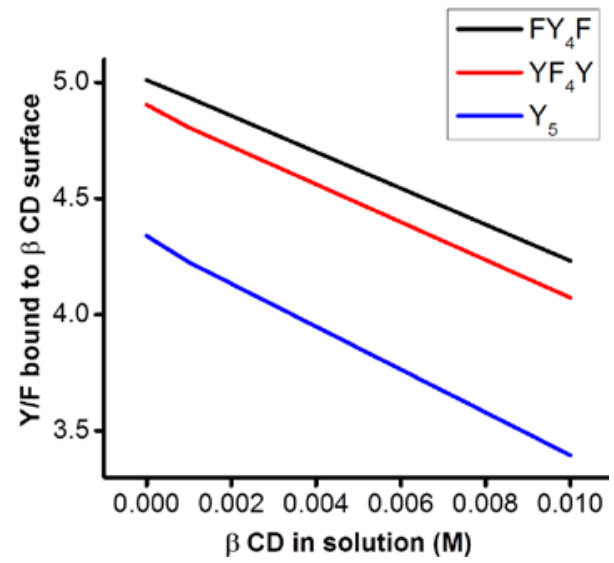

Figure 6.9 - Valency of interaction in peptide as a function of surface coverage at various $\beta$-CD concentrations.

Another interesting aspect is how the positioning of weak and strong binding sites in the peptides influences their spreading behavior. As discussed before, the affinity of phenylalanine to form a complex with $\beta$-CD is less compared to tyrosine. For peptides that contain only $\mathrm{Y}$ or $\mathrm{F}\left(\mathrm{Y}_{6}, \mathrm{Y}_{5}\right.$ and $\left.\mathrm{F}_{6}\right)$, there appears to be always a slight preference for the binding of ligand sites located at the core of the peptide to the $\beta$-CD surface compared to terminal sites. This is a statistical effect because a core site has two direct 
neighbors which can bind with a high $E M$, while the terminal sites have only one. Because binding $\beta$-CD from solution has no statistical preference, it binds (slightly) easier at the ends, and therefore the preference for using core sites to bind to the surface is slightly higher when increasing the $\beta$-CD concentration in solution. For $F Y_{4} F$, this effect is diminished (preference for core binding remains constant) and for $\mathrm{YF}_{4} \mathrm{Y}$, this effect is (slightly) enhanced (more preferred core binding, because terminal Y sites are more prone to $\beta$-CD binding from solution). Y sites bind stronger than $\mathrm{F}$ sites, both to $\beta$ CD surface sites as well as solution $\beta$-CD. Therefore, the effect of competition is seen more in peptides that are rich in Y. It should be noted that experimentally the difference in binding behavior is mainly observed in the onset of desorption, which does occur notably for $\mathrm{FY}_{4} \mathrm{~F}$, but not for $\mathrm{YF}_{4} \mathrm{Y}$. In contrast, the spreading rates of both peptides remain rather constant over a large concentration range, and the faster spreading peptide $\left(\mathrm{YF}_{4} \mathrm{Y}\right)$ is the one with the larger number of weak sites.

In contrast to the reported bis-Ad system, ${ }^{7}$ the large number of species and the complexity of the peptide systems prohibit the identification of a single or a few characteristic species to assign to the walking and hopping mechanisms (Figure 6.1b/c). The overall species distributions were changing upon increasing the $\beta$-CD concentration in solution, but both the surface-bound and unoccupied free sites of the peptides decreased. If we take the ratio between free and surface-bound sites as a measure for dynamicity (as discussed above in the section related to Figure 6.8, this ratio is roughly $0.02 / 100=0.0002$ for bis-Ad), the peptides are clearly more dynamic (ratios are typically between 0.2-0.3). The ratio is higher and more constant for F-containing peptides, and a bit lower and decreasing for Y-containing peptides. This may indicate that one can expect rather constant dynamics (as observed experimentally, see Figure 6.4), and maybe a bit slowing down of walking at higher $\beta$-CD in solution.

Similar to the mechanisms discussed before for the bis-Ad system, hopping of the peptides occurs by dissociation of monovalently bound species and rebinding of the complementary solution species which have at least one guest site free for interaction to a surface $\beta$-CD site (Figure 6.1c). Since the peptides keep, on average, several guest sites uncomplexed to $\beta$-CD from solution, rebinding remains highly favored, which is in sharp contrast to the bis-Ad system. 


\subsection{Conclusions}

In this chapter, the surface spreading of fluorescent penta- and hexavalent peptides in the presence of various concentrations of $\beta-C D$ in the surrounding solution has been studied. A complex spreading pattern of these peptides on the surface was observed. Several parameters were found to influence the spreading of the multivalent ligands on the $\beta$-CD receptor surface. In general, the valency of a peptide was found to influence the spreading to a larger extent than the individual contributions of the binding motifs. the pentavalent peptide $\left(\mathrm{Y}_{5}\right)$ showed desorption at higher $\beta$-CD concentrations, and therefore spreading by the flying mechanism, which was delayed in the hexavalent peptides $\left(\mathrm{YF}_{4} \mathrm{Y}, \mathrm{FY}_{4} \mathrm{~F}\right)$. For the hexavalent guests, the peptide with the weaker core $\left(\mathrm{YF}_{4} \mathrm{Y}\right)$ showed a higher spreading rate at low $\beta$-CD concentrations in solution compared to the one $\left(\mathrm{FY}_{4} \mathrm{~F}\right)$ with the stronger core. Both thermodynamic and kinetic parameters, such as rebinding, were found to contribute to the differences in behavior. Moreover, the presence of $\mathrm{F}$ in the hexavalent peptides led to a decrease of their probability to bind to $\beta$-CD in solution, and thus to a decreased sensitivity to competition. Thus we found that an increase of the number of $\mathrm{F}$ sites in a peptide (from $\mathrm{FY}_{4} \mathrm{~F}$ to $\mathrm{YF}_{4} \mathrm{Y}$ ), the sensitivity of the multivalent peptide to desorb from the surface was decreased. Improved understanding of the molecular motion of our biomimetic multivalent constructs can help to unravel some key mechanisms of ligand-receptor interactions at biological interfaces, and might find application in molecular medicine.

\subsection{Acknowledgements}

Gulistan Kocer is acknowledged for her help with the FRAP measurements. Dr. Damien Thompson and Dr. Pierre-Andre Cazade are acknowledged for their help with current ongoing molecular dynamics simulations. Shrikrishnan Sankaran is acknowledged for help with the conceptual figure.

\subsection{Materials and methods}

General. Chemicals were obtained from commercial sources and used as received. Fmoc-Rink Amide MBHA resin (0.52 mmol/g), Fmoc-protected amino acids and related coupling agents for peptide synthesis were purchased from Multisyntech GmbH 
Chemicals. Maleimide-terminated Oregon green-488 (OG) dye was purchased from life technologies. Solvents employed in the purification of peptides were purchased from Sigma-Aldrich.

Equipment. A JMS-T100LC mass spectrometer was used to determine the mass of the peptides. High-performance liquid chromatography (HPLC) experiments were performed with equipment from Waters. Preparative column (Waters 2535 quaternary gradient module with $\mathrm{XBridge}^{\mathrm{TM}}$ prep C18 $5 \mu \mathrm{m} \mathrm{OPD}^{\mathrm{TM}} 19 \times 250 \mathrm{~mm}$ preparative column)with eluents $0.1 \%$ aqueous TFA and $0.1 \%$ TFA in acetonitrile were used to purify crude peptide. Extent of purification was checked using an analytical HPLC (Waters 2535 quaternary gradient module XBridge C18 $5 \mu$ m column with $4.6 \times 250$ mm dimensions). Same eluents as that of preparative HPLC were used. HPLC retention times were observed following analytical HPLC with a solvent gradient of $0-90 \%$ acetonitrile over 90 min. Mass of purified peak was determined using LC-MS (Waters 2535 module coupled to micromass LCT))

Synthesis. Peptides were synthesized by solid phase peptide synthesis using Fmoc-Rink Amide MBHA resin, following standard Fmoc protocols. Purification of the peptide was achieved with preparative reversed phase HPLC. The purity of the peptides was analyzed by reverse phase analytical HPLC and ESI mass spectra.

\section{Conjugation of fluorescent dye to a peptide}

The coupling of the OG dye to the peptides was carried out in an oxygen free environment by continuously blowing nitrogen through the solvent. Cysteine-terminated peptides were dissolved in degassed phosphate (PBS) buffer at approx. $1 \mathrm{mg} / \mathrm{ml}$ concentration. The solution was then kept aside for 30 min under continuous flow of nitrogen. Afterwards 100 equiv of TCEP (180 $\mu$ g in $10 \mu \mathrm{l}$ per mg of peptide) was added to the solution and mixed thoroughly. The reaction was then incubated at room temperature for $10 \mathrm{~min}$. The dye solution was prepared in $50 \mu \mathrm{l}$ of anhydrous dimethylformamide and added to the peptide solution. The reaction mixture was immediately flushed with nitrogen and mixed thoroughly for $2 \mathrm{~h}$ at room temperature. The reaction mixture was left overnight at $2-8^{0} \mathrm{C}$. Thereafter, the reaction was subjected 
to purification with preparative reverse phase HPLC and the purity was determined using analytical HPLC.

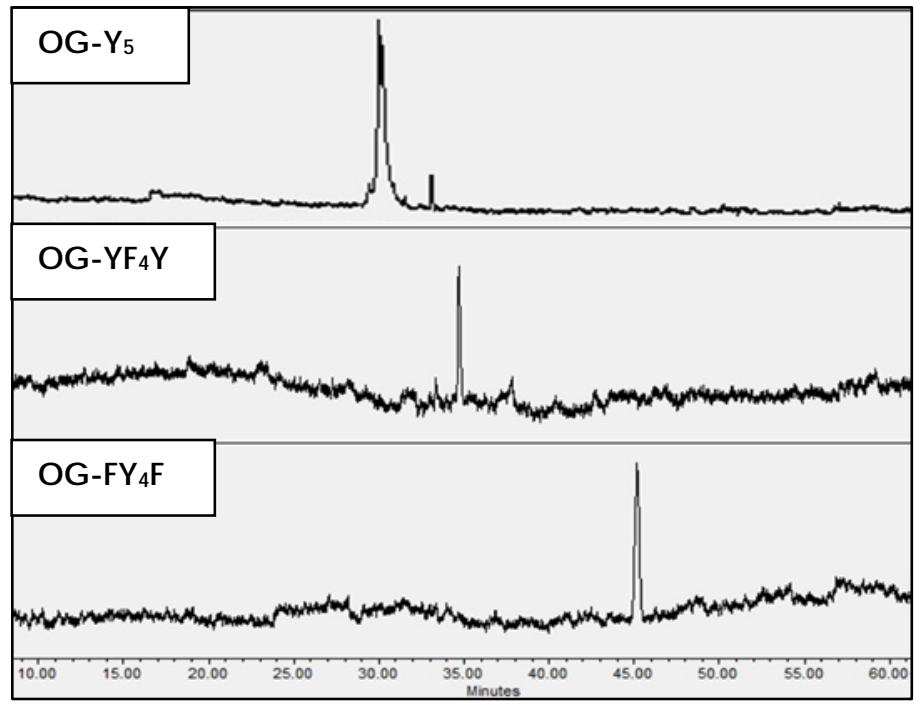

Figure 6.10 - Analytical HPLC traces of purified dye-coupled peptides monitored at $280 \mathrm{~nm}$.

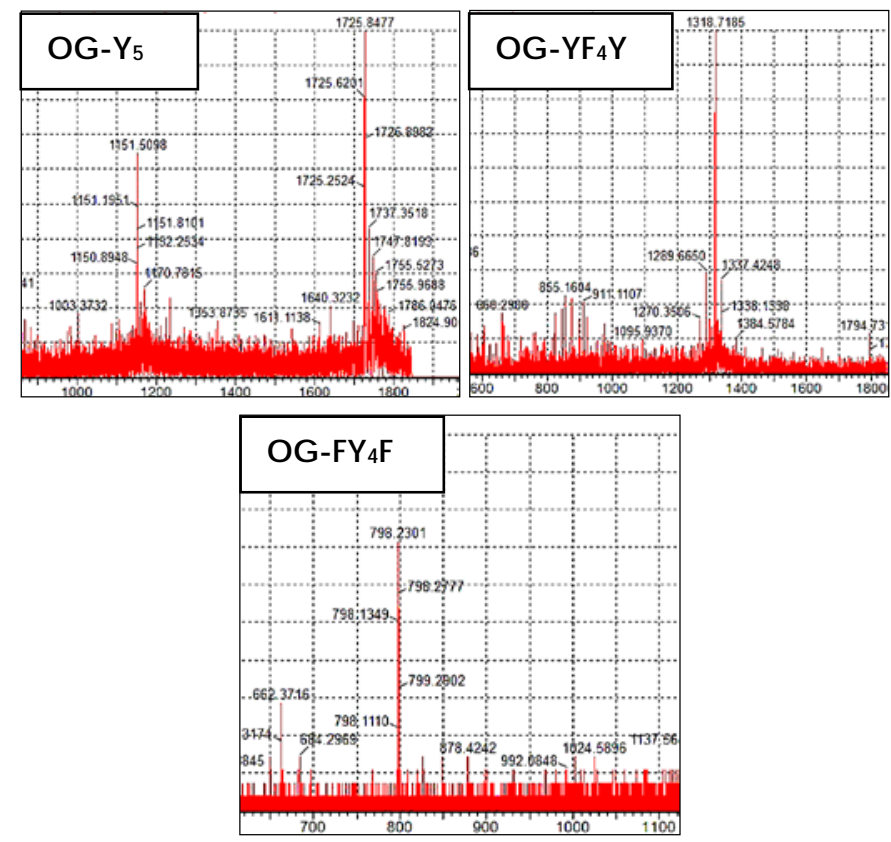

Figure 6.11 - Mass spectra of purified dye-coupled peptides. 
The reaction was then incubated at room temperature for $10 \mathrm{~min}$. The dye solution was prepared in $50 \mu \mathrm{l}$ of anhydrous dimethylformamide and added to the peptide solution. The reaction mixture was immediately flushed with nitrogen and mixed thoroughly for $2 \mathrm{~h}$ at room temperature. The reaction mixture was left overnight at $2-8^{0} \mathrm{C}$. Thereafter, the reaction was subjected to purification with preparative reverse phase HPLC and the purity was determined using analytical HPLC.

\section{OG-C(SGGYGGS)}

OG-C(SGGYGGS) 5 was synthesized and purified, with a yield of $1.21 \mathrm{mg}, 0.61 \%$. ESI mass spectra of OG-C(SGGYGGS $)_{5}$ confirmed the successful synthesis of the peptide. Chemical Formula: $\mathrm{C}_{145} \mathrm{H}_{177} \mathrm{~F}_{2} \mathrm{~N}_{37} \mathrm{O}_{59} \mathrm{~S}$, Mw: $3452.23 \mathrm{~g} / \mathrm{mol}$. $[\mathrm{M}+\mathrm{H}]^{2+}$ : calculated: 1727.13; found: 1726.89; $[\mathrm{M}+\mathrm{H}]^{3+}$ : calculated: 1151.74; found: 1151.50 . The analytical HPLC showed a single peak, indicating product of high purity ( $>90 \%)$.

\section{OG-CSGGFGGS(SGGYGGS) ${ }_{4}$ SGGFGGS}

Peptide OG-CSGGFGGS(SGGYGGS) ${ }_{4}$ SGGFGGS was synthesized and purified, with a yield of $1.57 \mathrm{mg}, 0.79 \%$. ESI mass spectra of OG-CSGGFGGS (SGGYGGS) SGGFGGS confirmed the successful synthesis of the peptide. Chemical Formula: $\mathrm{C}_{168} \mathrm{H}_{208} \mathrm{~F}_{2} \mathrm{~N}_{44} \mathrm{O}_{67} \mathrm{~S}, \mathrm{Mw}: 3985.77 \mathrm{~g} / \mathrm{mol}$. [M+H] ${ }^{5+}$ : calculated: 798.15; found: 798.23. The analytical HPLC showed a single peak, indicating a product of high purity ( $>90 \%)$.

\section{OG-CSGGYGGS(SGGFGS) 4 SGGYGGS}

Peptide OG-CSGGYGGS(SGGFGGS) ${ }_{4}$ SGGYGGS was synthesized and purified, with a yield of $1.38 \mathrm{mg}, 0.69 \%$. ESI mass spectra of OG-CSGGYGGS(SGGFGGS) SGGYGGS confirmed the successful synthesis of the peptide. Chemical Formula: $\mathrm{C}_{168} \mathrm{H}_{208} \mathrm{~F}_{2} \mathrm{~N}_{44} \mathrm{O}_{65} \mathrm{~S}, \mathrm{Mw}$ : 3953.77. [M+H] $]^{3+}$ : calculated: 1318.92; found: 1318.71. The analytical HPLC showed a single peak, indicating product of high purity (>90\%). 


\section{Substrate preparation}

SAMs of $\beta$-cyclodextrin on glass were prepared by a method reported by our group previously. ${ }^{8}$ Patterning of the molecular printboard with the fluorescent guest molecules was achieved using microcontact printing as reported previously. ${ }^{6}$

\section{Fluoroscence recovery after photobleaching (FRAP) measurements}

FRAP measurements were conducted using a Nikon A1 CLSM with a 20x objective. Data was corrected for acquisition bleaching and normalized. Diameter of the bleaching spot $(9.98 \mu \mathrm{m})$ was kept constant during all the measurements. We immersed the $\beta$-CD surface in a solution of $30 \mu \mathrm{M}$ fluorescent peptide for 15 min followed by washing with water to remove any unbound peptide from the surface. The surface was then sealed with rubber ring followed by incubation with $\beta$-CD solution, samples were then immediately subjected to FRAP measurement.

\section{FRAP data to spreading rate}

In order to convert the FRAP data to the spreading rates, a plot of one-dimensional diffusion vs time was simulated with an arbitrary $D$ value using Fick's diffusion law.

$$
n(x, t)=n_{0} \operatorname{erfc}\left(x /\left(2(D t)^{1 / 2}\right)\right)
$$

$n(x, t)$ represents the concentration of molecules at position $x$ and time $t$ from a boundary located at position $x=0$, where the concentration is maintained at a value $n_{0}$, erfc is complementary error function, and $D$ is a diffusion constant. The time points that are used to simulate the diffusion match that of the observation times in FRAP and in the printing experiments (0-40 min). The values of distance $x$ where $n=1 / 2 n_{0}$ where plotted as a function of time (Figure 6.10), and fitted to a linear equation using the timepoints between 10-40 min. The slope of these lines is taken as the spreading rate. This gave an empirical relationship (spreading rate $(\mathrm{nm} / \mathrm{s})=12.6 \times \sqrt{ } D\left(\mathrm{um}^{2} / \mathrm{s}\right)$ ) between the spreading rate and the diffusion constant $(D)$. The apparent $D$ values measured by FRAP where converted into spreading rates using this formula, thus leading to the spreading rate values shown in Table 6.1. 
a

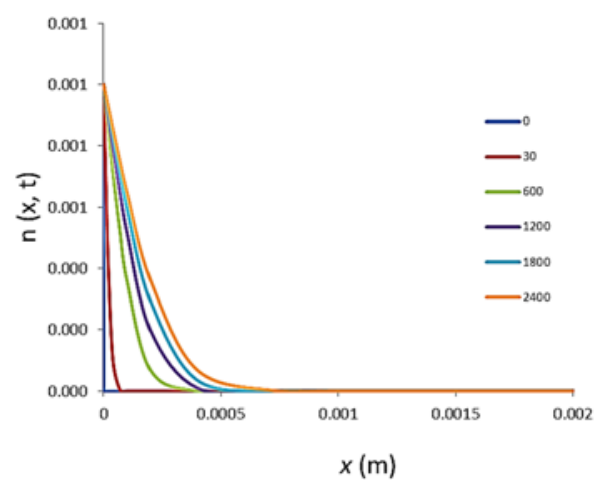

b

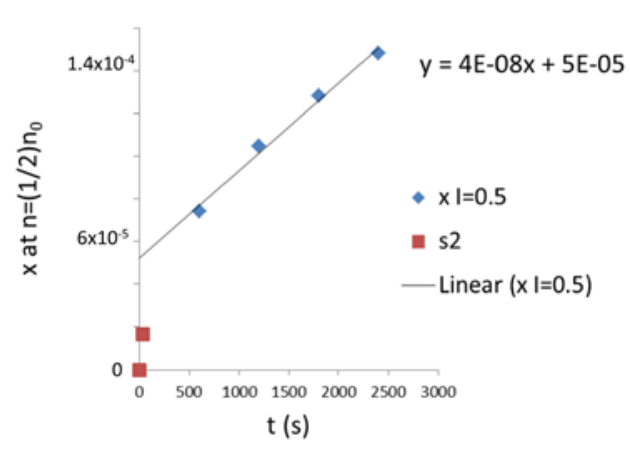

Figure 6.13 - a) Simulated graph showing change in $n(x, t)$ as a function of distance and time (0$2400 \mathrm{~s}$ ) for a diffusion constant $D=10^{-11} \mathrm{~m}^{2} / \mathrm{s}$. b) Simulated plot of $x$ at $n=1 / 2 n_{0}$ as a function of time, and a linear fit to the data points at $\mathrm{t}=10-40 \mathrm{~min}$.

\subsection{References}

1. Kiessling, L.L., Gestwicki, J.E. \& Strong, L.E. Synthetic multivalent ligands as probes of signal transduction. Angewandte Chemie International Edition 45, 2348-2368 (2006).

2. Mammen, M., Choi, S.-K. \& Whitesides, G.M. Polyvalent interactions in biological systems: implications for design and use of multivalent ligands and inhibitors. Angewandte Chemie International Edition 37, $2754-2794$ (1998).

3. Grunwald, D., Singer, R.H. \& Rout, M. Nuclear export dynamics of RNAprotein complexes. Nature 475, 333-341 (2011).

4. von Delius, M., Geertsema, E.M. \& Leigh, D.A. A synthetic small molecule that can walk down a track. Nature Chemistry 2, 96-101 (2010).

5. Schleicher, K.D. et al. Selective transport control on molecular velcro made from intrinsically disordered proteins. Nature Nanotechnology 9, 525-530 (2014).

6. Huskens, J. Diffusion: Molecular velcro in Flatland. Nature Nanotechnology 9, 500-502 (2014). 
7. Perl, A. et al. Gradient-driven motion of multivalent ligand molecules along a surface functionalized with multiple receptors. Nature Chemistry 3, 317-322 (2011).

8. Tito, N.B. \& Frenkel, D. Optimizing the selectivity of surface-adsorbing multivalent polymers. Macromolecules 47, 7496-7509 (2014).

9. Rekharsky, M. \& Inoue, Y. Chiral recognition thermodynamics of $\beta$ cyclodextrin: the thermodynamic origin of enantioselectivity and the enthalpy-entropy compensation effect. Journal of the American Chemical Society 122, 4418-4435 (2000).

10. Sadhu, V.B., Perl, A., Duan, X., Reinhoudt, D.N. \& Huskens, J. Supramolecular microcontact printing with receptor-functionalized PDMS stamps. Soft Matter 5, 1198-1204 (2009).

11. Sprague, B.L. \& McNally, J.G. FRAP analysis of binding: proper and fitting. Trends in Cell Biology 15, 84-91 (2005).

12. Kang, M., Day, C.A., Kenworthy, A.K. \& DiBenedetto, E. Simplified equation to extract diffusion coefficients from confocal frap data. Traffic 13, 1589-1600 (2012).

13. Huskens, J. et al. A model for describing the thermodynamics of multivalent host-guest interactions at interfaces. Journal of the American Chemical Society 126, 6784-6797 (2004).

14. Lagerholm, B.C. \& Thompson, N.L. Theory for ligand rebinding at cell membrane surfaces. Biophysical Journal 74, 1215-1228 (1998). 


\title{
Chapter 7
}

\section{Modulating the nucleated self-assembly of tri- $\beta^{3}$ - peptides using cucurbit[n]urils}

\begin{abstract}
The hierarchical nucleated self-assembly of tri- $\beta^{3}$-peptide has been elucidated using circular dichroism spectroscopy (CD), dynamic light scattering (DLS), atomic force microscopy (AFM) and scanning electron microscopy (SEM). Modulation of the assembly process through interactions between cucurbit[n]urils $(\mathrm{CB}[\mathrm{n}] \mathrm{s})$ and $\beta$ tyrosines in a tri- $\beta^{3}$-peptide is dependent on the size of $\mathrm{CB}[\mathrm{n}]$. While formation of larger aggregates can be effectively inhibited by CB[7], its larger family member CB[8] promoted the assembly of protofibrils into larger aggregates. General insight from differentially modulating supramolecular assembly can lead to new ways to change properties of supramolecular polymers.
\end{abstract}




\subsection{Introduction}

Nucleated self-assembly is the most prominent mechanism involved in the fibrillization of proteins observed in various disease conditions. ${ }^{1}$ For example, the process of prion infection is characterized by the conversion of normal host-coded proteins into abnormal misfolded $\beta$-sheet assemblies. These $\beta$-sheets then hierarchically assemble into dimers, tetramers, protofibrils and finally large fibrillar aggregates. ${ }^{2}$ Various synthetic peptides and other molecular systems self-assemble into fibrillar structures mimicking many aspects of nucleated assembly of proteins observed in nature. ${ }^{3-8}$ As expected, the nucleated aggregation of peptides requires distinct sequence motifs and their aggregation can be modulated using conventional factors such as concentration, $\mathrm{pH}$, time and temperature. More interestingly, the onset and regulation of peptide assembly can be activated by light or enzymatic switches. ${ }^{9-10}$

In spite of these advances, the programmable aggregation of peptides and molecules in a hierarchical manner remains elusive. In particular, recent research has demonstrated that the addition of chiral auxiliaries or seed molecules can lead to either the exclusive formation of metastable helical aggregates or allows control over fibrillar width and length as shown in mechanistic assembly studies on aromatic disc- and rod-like molecules. ${ }^{11-15}$ Promising results have also been reported by Moore and co-workers to control the final outcome of the nucleated assembly of $\alpha$-peptides by the addition of polymer-peptide conjugates into discrete nanostructures. ${ }^{16}$ Very recently, the addition of macrocycles $\mathrm{CB}[7]$ and $\mathrm{CB}[8]$ assisted the assembly of functional dimeric and tetrameric proteins, protein wires and cell clusters mediated by interactions of these macrocycles with aromatic amino acids in proteins. ${ }^{17-22} \mathrm{Kim}$ and co-workers interestingly achieved inhibition of $\alpha$-peptide fibril formation by specific $\mathrm{CB}[7]$ phenylalanine interactions. $^{23}$

$\alpha$-Peptides composed of less than 15 amino acids generally do not adopt defined helical conformations, in absence of structural constraints. In strong contrast, a surprising aspect of $\beta$-peptides is that they adopt defined helical structures over very short sequences despite the presence of the additional methylene units, which would be expected to provide the backbone with an increased freedom of orientation. ${ }^{24-25}$ Therefore, $\beta$-peptides have evolved as an intensively investigated class of nonbiological building blocks for new materials, catalysts and ligands for protein 
receptors. ${ }^{26-31}$ To the best of our knowledge the assembly mechanism of short $\beta$-peptides has not been reported so far, while there are no attempts to modulate their assembly by addition of molecular components.

Here we report the nucleated self-assembly of tri- $\beta^{3}$-peptides, composed of three $\beta$ amino acids, into supramolecular fibrils. The modulating capability of the $\mathrm{CB}[\mathrm{n}] \mathrm{s}$ on the nucleated self-assembly of tri- $\beta^{3}$-peptides was also investigated. We found that by varying the cavity size from $\mathrm{CB}[7]$ to $\mathrm{CB}[8]$ distinct phases of assembling tri- $\beta^{3}$ peptides can be arrested (Scheme 1). Given the limited size of the CB[7] cavity, only one aromatic amino acid can be simultaneously hosted and hence CB[7] is primarily acting as an inhibitor of self-assembly. In strong contrast, the larger $\mathrm{CB}[8]$ can form a ternary complex with two aromatic amino acids and hence $\mathrm{CB}[8]$ is acting primarily as cross-linker of multiple fibers.

\subsection{Results and discussion}

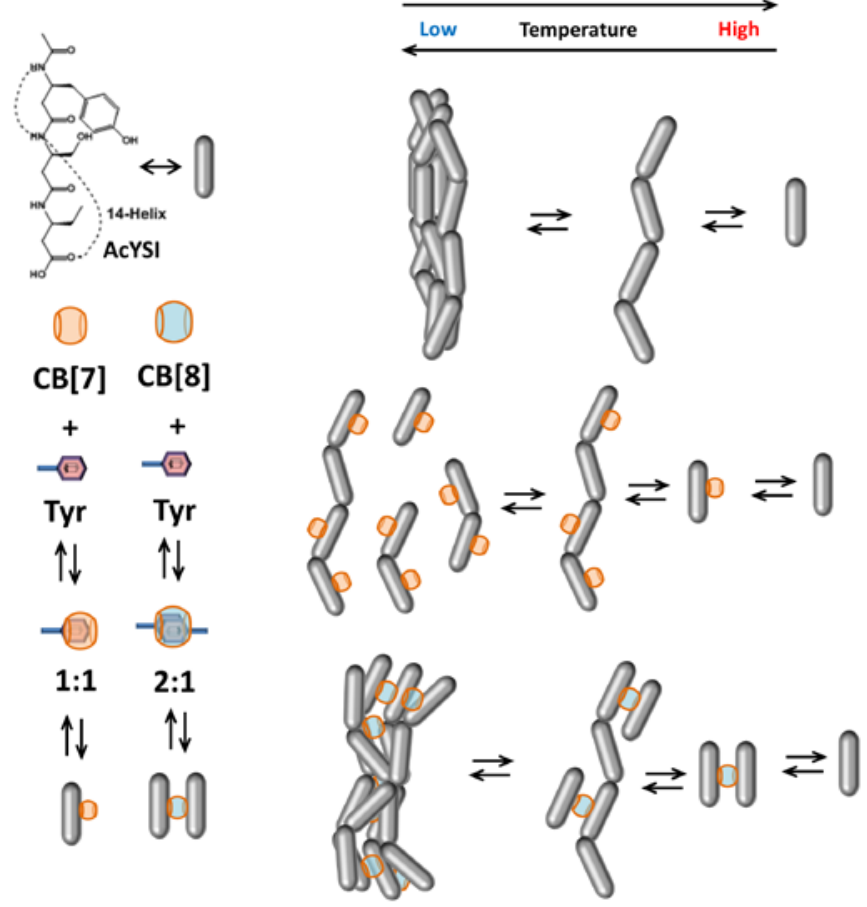

Figure 7.1 - Hierarchical assembly of AcYSI 
A tri- $\beta^{3}$-peptide (AcYSI, Scheme 7.1) was synthesized following standard procedures (details in materials and methods section). This short $\beta$-peptide is known to exhibit six axially oriented hydrogen bonding interactions facilitating the axial self-assembly during fiber formation as reported previously by others. ${ }^{28}$ In the peptide design, $\beta$ isoleucine facilitates aggregation by hydrophobic interactions, while $\beta$-serine enhances water solubility. The N-terminus is acetylated (Ac) to prevent formation of charges. Finally, the presence of $\beta$-tyrosine in the tri- $\beta^{3}$-peptide further stabilizes the selfassembled structures via $\pi-\pi$ stacking leading to the formation of larger fibrillar structures. $^{28}$

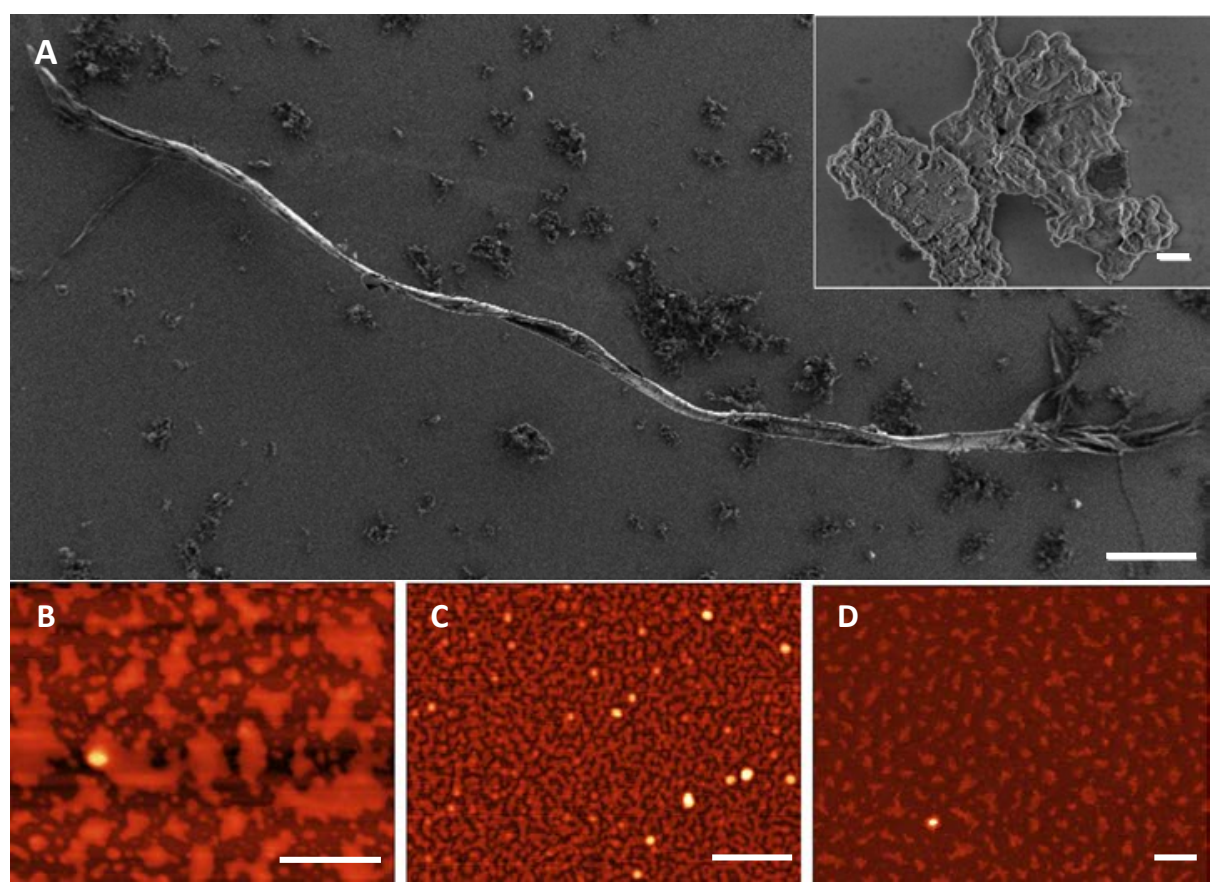

Figure 7.2 - (A) SEM images of AcYSI (3.9 mM) and CB[8] (34 $\mu \mathrm{M})$ and (inset) AcYSI (3.9 $\mathrm{mM}$ ) and CB[7] (34 $\mu \mathrm{M})$. AFM images of AcYSI alone: (B) $0.39 \mathrm{mM},(\mathrm{C}) 1.5 \mathrm{mM}$ and (D) 3.9 mM. Scale bars: (A) $100 \mu \mathrm{m}$; inset, (B), (C) and (D) $200 \mathrm{~nm}$.

Importantly, the $\beta$-tyrosine units also provide a handle to control the assembly process through host-guest interactions with $\mathrm{CB}$ [7] and $\mathrm{CB}$ [8]. First, the morphological changes upon adding $\mathrm{CB}[\mathrm{n}] \mathrm{s}$ to the self-assembled structures of AcYSI were visualized using SEM, AFM and optical microscopy (Figure 7.2). All samples were heated to $90^{\circ} \mathrm{C}$ and 
cooled down to $20^{\circ} \mathrm{C}$ to facilitate the possible entry of $\mathrm{CB}[\mathrm{n}] \mathrm{s}$ within the fibrils during the re-assembly of the peptides before depositing them onto a surface. Inspection of small deposits of concentrated solutions of AcYSI (3.9 mM) on various surfaces showed twisted fibrillar assemblies of several micrometers in length as readily observed using an optical microscope (Figure 7.15, materials and methods) and in agreement with observations by others. ${ }^{28}$ In contrast, when $34 \mu \mathrm{M}$ of $\mathrm{CB}[7]$ was added to these AcYSI assemblies, only small ill-defined clusters were detected (Figure 7.2 A, inset), whereas larger fibrillar assemblies were observed upon the addition of $34 \mu \mathrm{M}$ of CB[8] (Figure 7.2 A). Interestingly, the fibrils in the presence of $\mathrm{CB}[8]$ were larger in width and showed a more extended layered structure (Figure 7.16A) in comparison to fibers consisting of AcYSI alone (Figure 7.16, materials and methods).

Upon diluting the solution of AcYSI (without $\mathrm{CB}[\mathrm{n}] \mathrm{s}$ ) to $0.39 \mathrm{mM}$ no fibrils were observed, and only ill-defined structures were observed using AFM (Figure 7.2 B), however, at an intermediate concentration of $1.5 \mathrm{mM}$, wormlike fibrils were observed across the sample (Figure 7.2C). While isolated large fibrils were present at high concentration (3.9 mM), as imaged with SEM and optical microscopy, the presence of smaller fibrils was detected in the background using AFM (Figure 7.2D). Based on these measurements, we conclude that the hierarchical assembly of these short tri- $\beta^{3}$ peptides follows three stages. At low concentration monomeric tri- $\beta^{3}$-peptides exist that predominantly form small fibrils at intermediate concentrations, while larger fibrils are formed at high concentrations. Dynamic light scattering (DLS) measurements verified the involvement of different length scales in the assembly of AcYSI (Figure 7.3). At a low concentration (0.99 $\mathrm{mM})$, no fibrils were detected, whereas at an intermediate concentration of AcYSI (2.1 mM) aggregates of $141 \mathrm{~nm}$ in size were detected. At a high concentration of AcYSI (3.9 mM) larger aggregates of $240 \mathrm{~nm}$ were found in addition. Similar observations were made in amyloid assembly studies where protofibrils of intermediate size fuse to form larger, mature fibrils. ${ }^{32}$ 


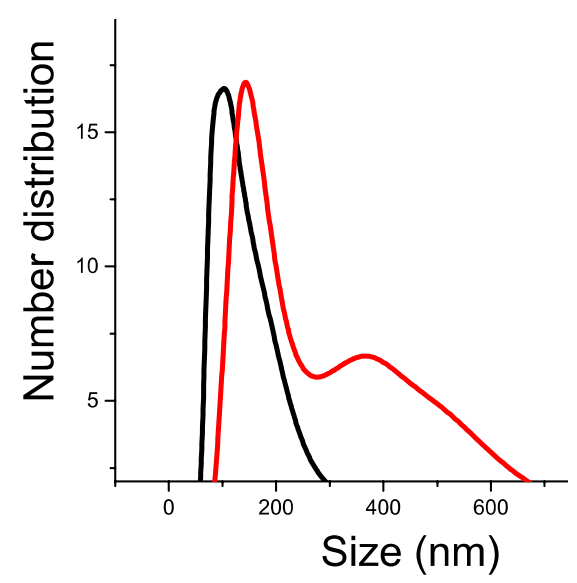

Figure 7.3 - DLS spectra of AcYSI at $2.1 \mathrm{mM}$ (black) and $3.9 \mathrm{mM}$ (red) concentration.

To gain further understanding into the mechanism of AcYSI assembly, CD spectroscopy was employed (Figure 7.4). No CD signal was observed in dimethyl sulfoxide and ethanol solutions up to a concentration of $3.9 \mathrm{mM}$ of AcYSI, indicating that these solvents do not support the assembly of AcYSI. When $0.30 \mathrm{mM}$ AcYSI solutions were prepared in PBS buffer also no CD signal was observed, indicative of the absence of folded tri- $\beta^{3}$-peptides. In contrast, upon increasing the concentration of AcYSI, the CD signal rose, while simultaneously the wavelength at which the CD intensity has its maximum value $\left(\lambda_{\max }\right)$ shifted bathochromically, signaling a gradual assembly of AcYSI into larger aggregates. A decrease in CD intensity and further red shift of $\lambda_{\max }$ was observed upon further increasing the concentration of AcYSI. This is in agreement with previous studies on $\alpha$-peptides that have shown that desolvation of tyrosine residues due to inclusion of the aromatic group in the hydrophobic core of the fiber during assembly leads to a gradual red shift of the CD spectra. ${ }^{33-34}$ Taken together, the changes in CD as a function of AcYSI concentration are supporting the AFM and SEM observations and indicate a hierarchical assembly process of individual monomeric tri- $\beta^{3}$-peptides (low concentration regime) into intermediate protofibrils (intermediate concentration regime) that interact to form mature fibrils (high concentration regime). 


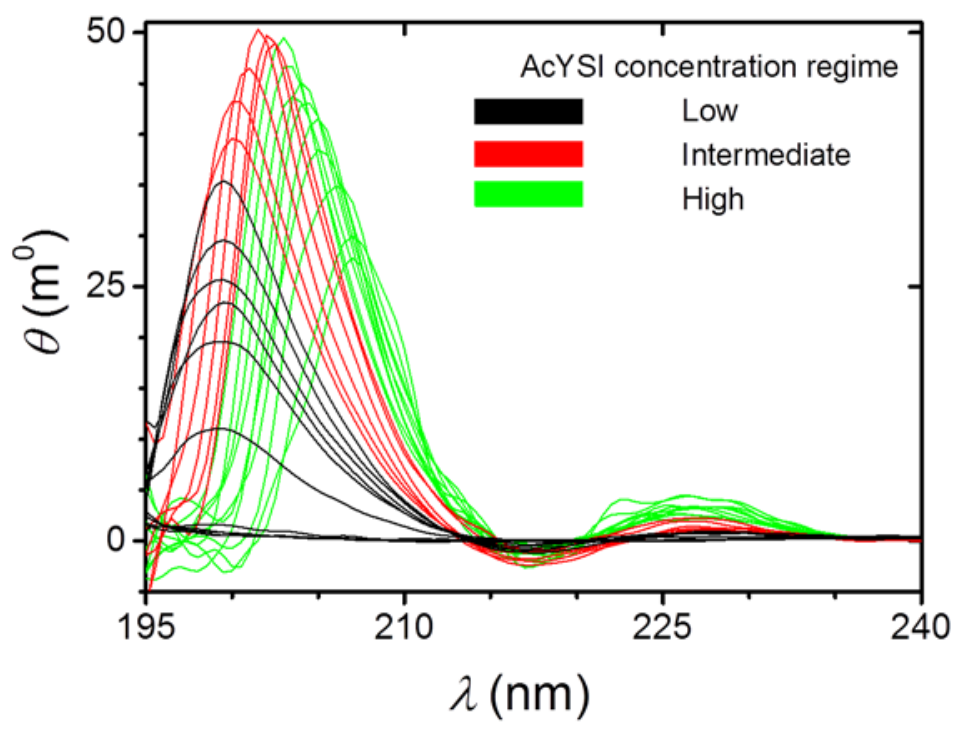

Figure 7.4 - CD spectra of AcYSI (PBS, $20^{\circ} \mathrm{C}$ ) at low (below $1.1 \mathrm{mM}$, black), high (above 2.1 $\mathrm{mM}$, green) or intermediate concentrations $(1.1-2.1 \mathrm{mM}$, red).

When $\lambda_{\max }$ is plotted against the AcYSI concentration (Figure 7.5), dilute solutions of AcYSI show no shift of this value up to a critical concentration of $1.1 \mathrm{mM}$ that marks a sudden change in $\lambda_{\max }$. This observation indicates the nucleation of the assembly process of helically folded peptides to form protofibrils. The concentration-dependent changes in this regime were analyzed using a nucleation-elongation model assuming a nucleus size of two tri- $\beta^{3}$-peptides, which reveals that the aggregate growth is highly cooperative. The growth of the protofibrils continues until $2.1 \mathrm{mM}$. At higher concentrations of AcYSI, up to $3.9 \mathrm{mM}$, changes in $\lambda_{\max }$ were monitored that deviate from the $1 \mathrm{D}$ growth model indicating substantial cross-linking of protofibrils. ${ }^{8}$ 


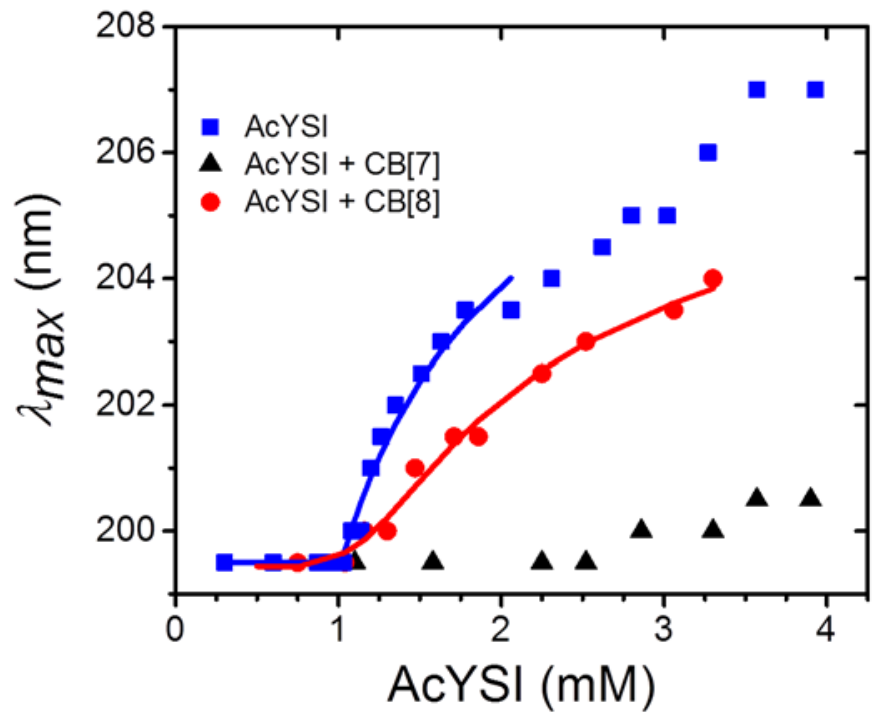

Figure $7.5-\lambda_{\max }$ of CD spectra (PBS, $20^{\circ} \mathrm{C}$, after heating) plotted vs AcYSI concentration (匹),

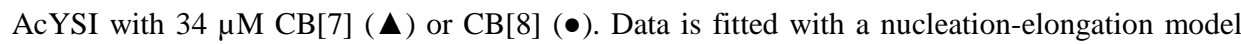
(lines).

Subsequently, the influence of temperature on the AcYSI assembly was investigated. To this end, AcYSI solutions were heated to $90^{\circ} \mathrm{C}$ and cooled down with $10^{\circ} \mathrm{C} / \mathrm{min}$ (Figures 7.6 A,B). In the case of high concentrations (above $2.1 \mathrm{mM}$ ) of AcYSI, CD spectra showed no change, indicating that the secondary structure of AcYSI at these concentrations are insensitive to temperature. Yet, in the case of intermediate concentrated AcYSI solutions (1.1 - $2.1 \mathrm{mM})$, no CD signal was found at $90^{\circ} \mathrm{C}$, indicating that protofibrils were disassembled, while from $70^{\circ} \mathrm{C}$ to $20^{\circ} \mathrm{C}$ the $\mathrm{CD}$ intensity gradually restored without hysteresis indicating that the protofibrils were completely re-assembled. Figure 7.7 shows for both concentration regimes a nearly temperature independent $\lambda_{\max }$. In the intermediate concentration regime (Figure 7.7, black), where AcYSI is in the protofibrillar state up to $70^{\circ} \mathrm{C}, \lambda_{\max }$ is $201.5 \mathrm{~nm}$, whereas in the high concentration regime (Figure 7.7, red), where AcYSI is in mature fibril state, $\lambda_{\text {max }}$ is $207 \mathrm{~nm}$. 

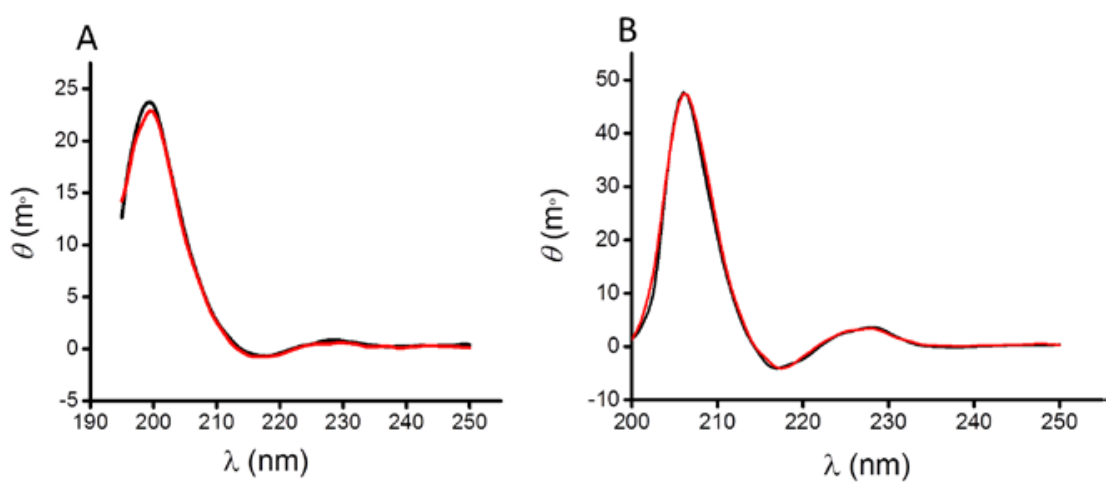

Figure 7.6 - CD spectra of (A) AcYSI $(1.1 \mathrm{mM})$ recorded at $20^{\circ} \mathrm{C}$ (black line) and after (red line) a temperature cycle from $20^{\circ} \mathrm{C}$ up to $90^{\circ} \mathrm{C}$ and down to $20^{\circ} \mathrm{C}$ at $10^{\circ} \mathrm{C} / \mathrm{min}$ (B) CD spectra of AcYSI (3.9 mM) recorded at $20^{\circ} \mathrm{C}$ (black line) and after (red line) a temperature cycle from $20^{\circ} \mathrm{C}$ up to $90^{\circ} \mathrm{C}$ and down to $20^{\circ} \mathrm{C}$ at $10^{\circ} \mathrm{C} / \mathrm{min}$.

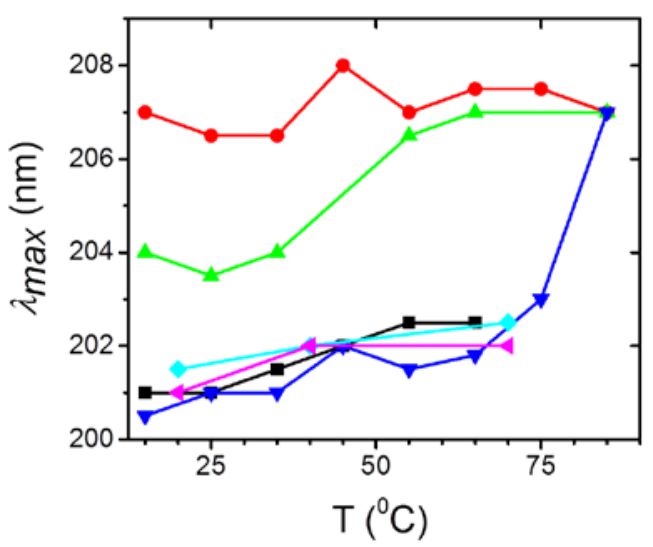

Figure $7.7-\lambda_{\max }$ of CD spectra plotted vs temperature for $1.1 \mathrm{mM}(\boldsymbol{\bullet})$ and $3.9 \mathrm{mM}(\bullet)$ AcYSI;

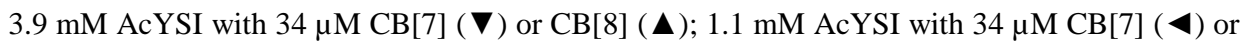
$\mathrm{CB}[8](\bullet)$.

Next, either $34 \mu \mathrm{M}$ of $\mathrm{CB}[7]$ or $\mathrm{CB}[8]$ was added to AcYSI in the protofibrillar state at $20^{\circ} \mathrm{C}$. No significant shift in $\lambda_{\max }$ was observed when compared to spectra of AcYSI alone (Figure 7.8 A, B). When CB[n]s were added to AcYSI in the disassembled state at $90^{\circ} \mathrm{C}$, after cooling down at $10^{\circ} \mathrm{C} / \mathrm{min}$, the melting curve closely resembled that of 
AcYSI alone (Figure 7.8 A, B, magenta and light blue). These results indicate that the macrocycles are not changing the protofibrillar assembly of AcYSI.
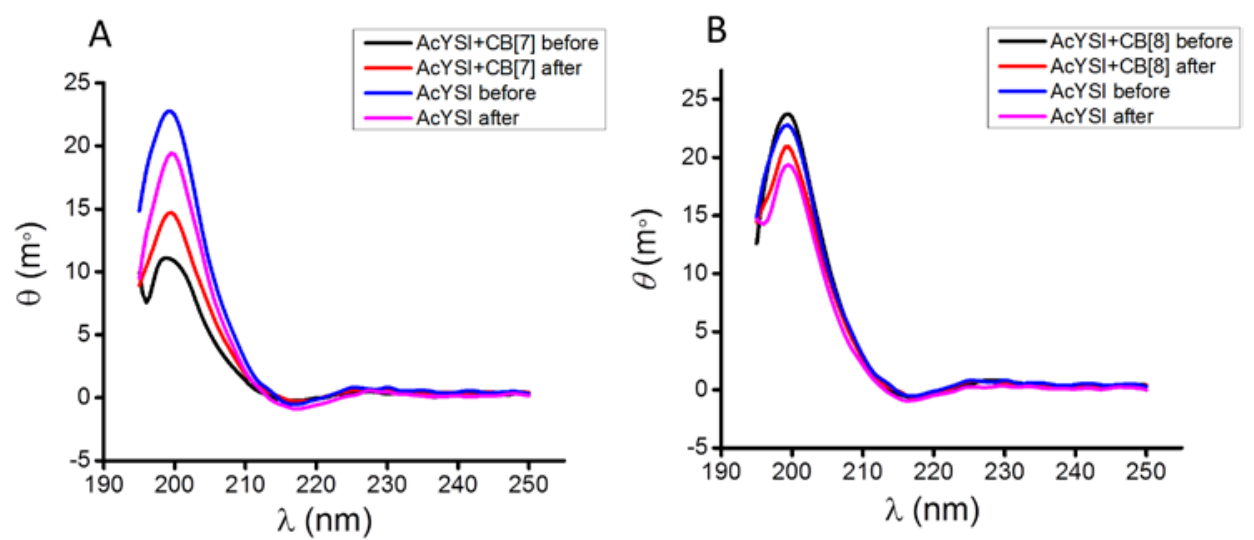

Figure 7.8 - CD spectra of A) AcYSI (1.1 mM); AcYSI (1.1 mM) and CB[7] (34 $\mu \mathrm{M})$ before and after temperature ramping from $20^{\circ} \mathrm{C}$ to $90^{\circ} \mathrm{C}$ and back to $20^{\circ} \mathrm{C}$, B) AcYSI (1.1 mM); AcYSI (1.1 mM) and $\mathrm{CB}[8](34 \mu \mathrm{M})$ before and after temperature ramping from $20^{\circ} \mathrm{C}$ to $90{ }^{\circ} \mathrm{C}$ and back to $20^{\circ} \mathrm{C}$.

To investigate the influence of $\mathrm{CB}[7]$ and $\mathrm{CB}[8]$ on the mature fibril state of AcYSI, 34 $\mu \mathrm{M}$ of $\mathrm{CB}[7]$ or $\mathrm{CB}[8]$ was added to AcYSI in the high concentration regime at $20^{\circ} \mathrm{C}$. Much to our surprise addition of $\mathrm{CB}[\mathrm{n}] \mathrm{s}$ lead to an immediate hypsochromic shift of $\lambda_{\max }$ for both samples (Figure 7.9 A,B) indicative of hindered maturation of fibrils by $\mathrm{CB}[7]$ and $\mathrm{CB}[8]$ as these $\mathrm{CD}$ spectra closely resemble that of AcYSI alone in the intermediate concentration regime where AcYSI is in the protofibrillar state (Figure 7.10). Interestingly, the spectra remained the same for at least $24 \mathrm{~h}$ and the shift in $\lambda_{\max }$ depends on the amount of macrocycles added. $\mathrm{A}$ CB[n]: $\beta$-Tyr ratio of $0.001(4 \mu \mathrm{M}$ $\mathrm{CB}[\mathrm{n}])$ appeared sufficient to modulate the assembly pathway (Figure 7.11 A,B). 

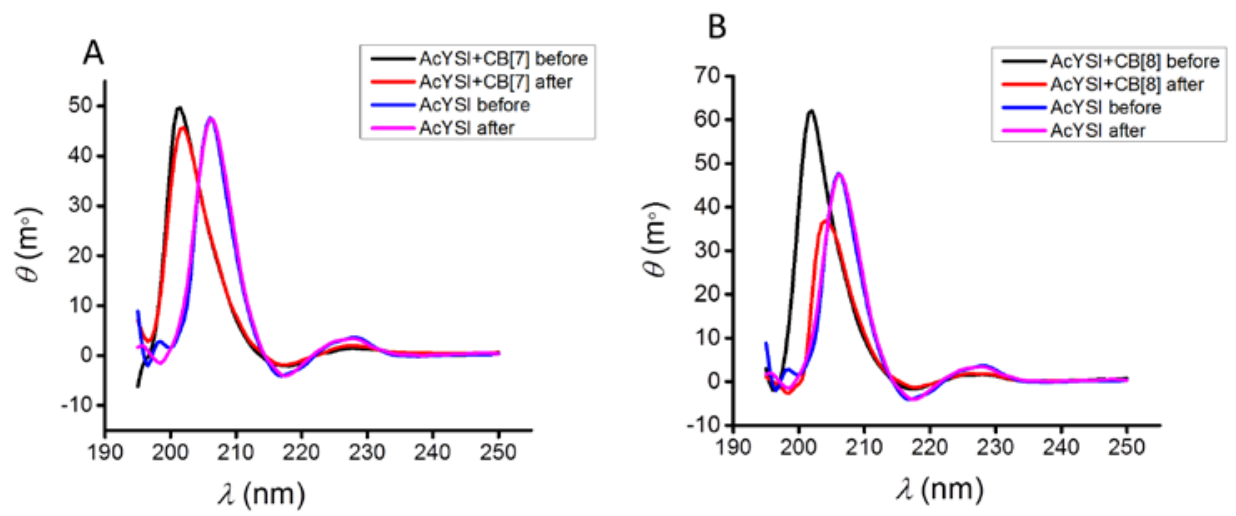

Figure 7.9 - A) AcYSI (3.9 mM); AcYSI $(3.9 \mathrm{mM})$ and CB[7] $(34 \mu \mathrm{M})$ before and after temperature ramping from $20^{\circ} \mathrm{C}$ to $90{ }^{\circ} \mathrm{C}$ and back to $20^{\circ} \mathrm{C}$ at the rate of $10{ }^{\circ} \mathrm{C} / \mathrm{min}$.B) AcYSI (3.9 mM); AcYSI (3.9 mM) and CB[8] $(34 \mu \mathrm{M})$ before and after temperature ramping from $20^{\circ} \mathrm{C}$ to $90{ }^{\circ} \mathrm{C}$ and back to $20^{\circ} \mathrm{C}$ at the rate of $10^{\circ} \mathrm{C} / \mathrm{min}$.

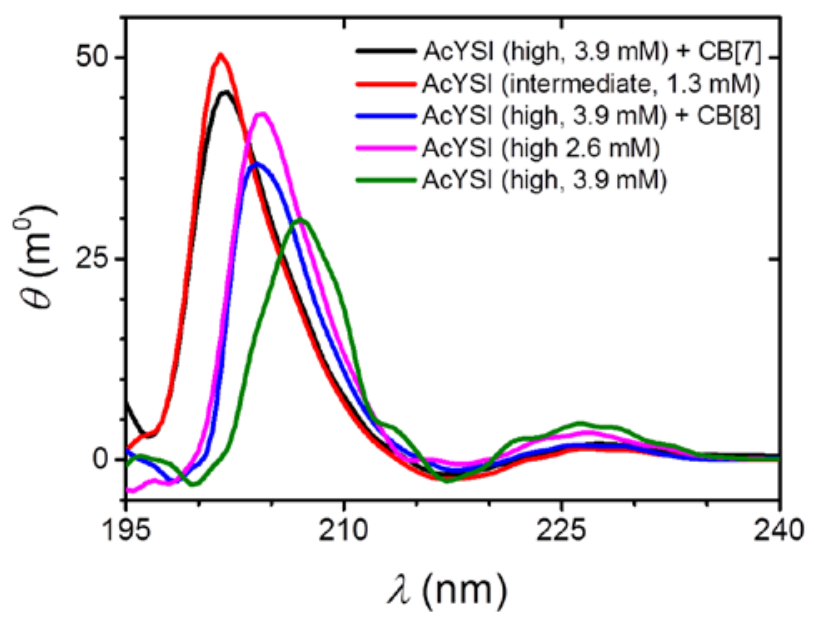

Figure 7.10 - CD spectra of AcYSI (3.9 mM), with $34 \mu \mathrm{M}$ CB[7] or $\mathrm{CB}[8]$ after heating to $90^{\circ} \mathrm{C}$ and cooling down to $20^{\circ} \mathrm{C}\left(10^{\circ} \mathrm{C} / \mathrm{min}\right)$. CD spectra of AcYSI $(1.3$ and $2.6 \mathrm{mM})$ are given for reference.

To examine whether kinetically trapped aggregates were formed, in the next experiment concentrated AcYSI solutions were heated to $90^{\circ} \mathrm{C}$ and in the presence of $34 \mu \mathrm{M} \mathrm{CB}$ [7] 
or $\mathrm{CB}[8]\left(\mathrm{CB}[\mathrm{n}]: \beta-\mathrm{Tyr}\right.$ ratio of 0.01 ) cooled down to $20^{\circ} \mathrm{C}$ at $10^{\circ} \mathrm{C} / \mathrm{min}$ (Figure 7.10 A,B). The same shift in $\lambda_{\max }$ of the $\pi-\pi^{*}$ transition was still observed for CB[7] (Figure 7.9 A), while, in strong contrast, for $\mathrm{CB}[8] \lambda_{\max }$ only shifted to $204 \mathrm{~nm}$ (Figure $7.9 \mathrm{~B}$ ) closely resembling one of the CD spectra of AcYSI in the high concentration regime. These results can be interpreted as follows. The CD spectrum of AcYSI ( $3.9 \mathrm{mM})$ in the presence of $\mathrm{CB}[8]$ and after heating is comparable to that AcYSI $(2.6 \mathrm{mM})$ in the absence of $\mathrm{CB}[8]$ (Figure 7.10), which indicates fibrils were formed with less intimate contacts compared to AcYSI alone at $3.9 \mathrm{mM}$.
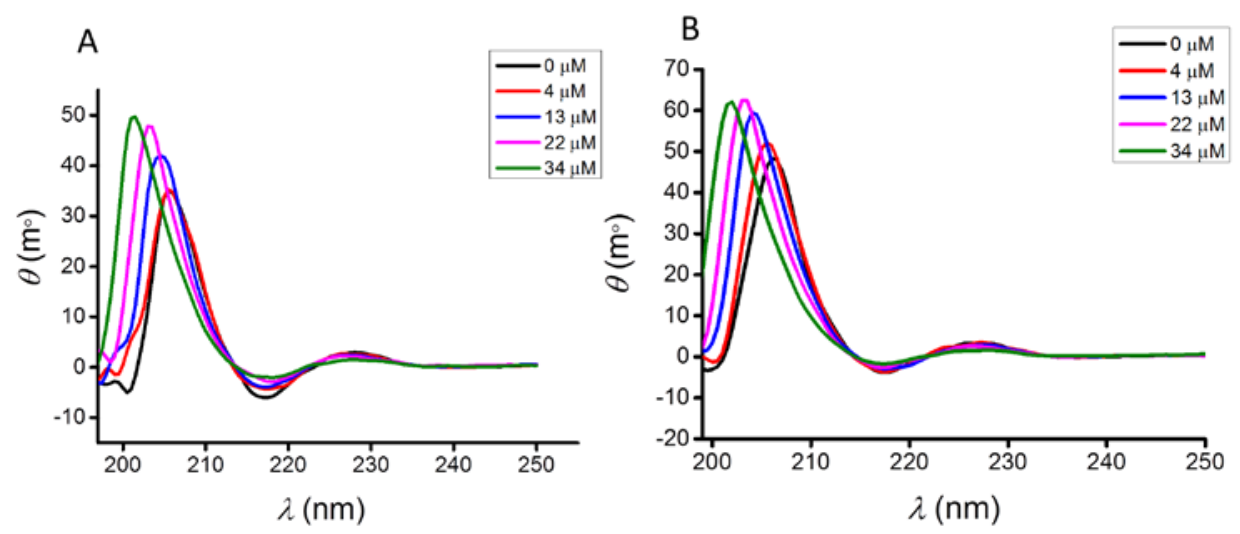

Figure 7.11 - CD spectra of (A) AcYSI (3.9 mM) and 0, 4, 13, 22 and $34 \mu \mathrm{M}$ of either (A) CB[7] or (B) $\mathrm{CB}[8]$.

This is in agreement with SEM images that showed a more extended layered fibril formation in the presence of $\mathrm{CB}[8]$ (Figure 7.16, materials and methods). These observations suggest that mature fibrils can form in the presence of $\mathrm{CB}[8]$, but that these fibrils are locally frustrated due to intercalation of $\mathrm{CB}[8]$, similar as observed previously in the case of assembling cross-linked rod-like molecules. ${ }^{13}$ When compared to the sample prior to heating, the CD spectrum of AcYSI and CB[8] resembles that of intermediate concentrations of AcYSI alone, indicative of the protofibrillar state (Figure 7.10). In contrast, in the case of $\mathrm{CB}$ [7] a much larger hypsochromic shift to $\lambda_{\max }=201$ nm was observed, indicating that $\mathrm{CB}[7]$ successfully suppresses the formation of mature fibrils, as witnessed by the match of $\lambda_{\max }$ with that of AcYSI alone at intermediate concentrations (Figure 7.10). These results clearly show that $\mathrm{CB}[7]$ and $\mathrm{CB}[8]$ have 
distinct influences on the assembly of AcYSI in the high concentration regime. In contrast to $\mathrm{CB}[8], \mathrm{CB}[7]$ can only bind a single tyrosine unit. As such it can inhibit the lateral assembly of protofibrils into mature fibrils thus arresting the assembly in the protofibrillar stage. Irrespective of temperature ramping $\mathrm{CB}$ [7] can stably arrest the protofibrillar state while $\mathrm{CB}[8]$ can cross-link these into larger fibrils depending on the equilibrating conditions.

To further investigate the modulation of the AcYSI assembly, CD spectra were measured of a series of solutions of AcYSI (0.59 to $3.9 \mathrm{mM})$ in the presence of CB[n] (CB[n]:AcYSI ratio remained constant, Figure 7.17, materials and methods). Figure 7.4 shows the change in $\lambda_{\max }$ against AcYSI concentration revealing that the presence of $\mathrm{CB}[\mathrm{n}] \mathrm{s}$ yields distinct shifts of $\lambda_{\max }$ with a strong difference between CB[7] and CB[8]. The assembly of AcYSI, in the presence of CB[8], nucleated at the same concentration as AcYSI alone, however the cooperativity is less. Also, the CD intensity is lower and $\lambda_{\max }$ is blue shifted indicating that complexation with $\mathrm{CB}[8]$ leads to less extended order of the fibrils, which is in agreement with SEM images in Figure 7.16. In the case of AcYSI in the presence of CB[7], nucleation-elongation is suppressed efficiently, only above $2.5 \mathrm{mM}$ some higher order assembly is taking place. Solutions of AcYSI with $\mathrm{CB}[8]$ also showed a markedly higher viscosity compared to solutions with $\mathrm{CB}$ [7] (Figure 7.12). This observation further corroborated that the macrocycles are interfering with the assembly of AcYSI, suggesting that, in the case of $\mathrm{CB}[8]$, a cross-linked network is formed that is not present in the case of CB[7]. This difference can be related to the possibility of $\mathrm{CB}[8]$ for binding two $\beta$-tyrosines from opposite sides of the cavity thus serving as a cross-linker between two protofibrils and yielding more viscous samples when compared to $\mathrm{CB}[7]$, in which case the mono-Tyr binding inhibits the lateral interaction of peptide fibrils. These observations are in good agreement with findings from the morphological study where large fibrils were observed in the case of $\mathrm{CB}[8]$, while in the presence of $\mathrm{CB}[7]$ no large fibrils were detected. 
A

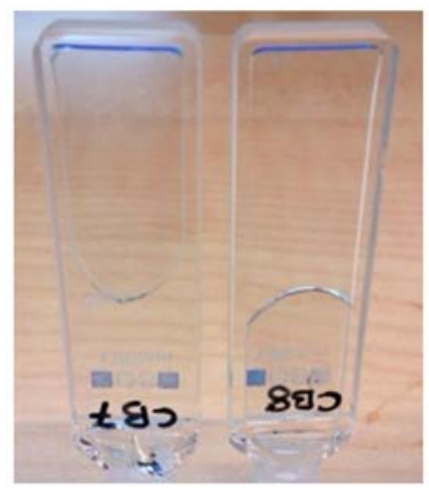

B

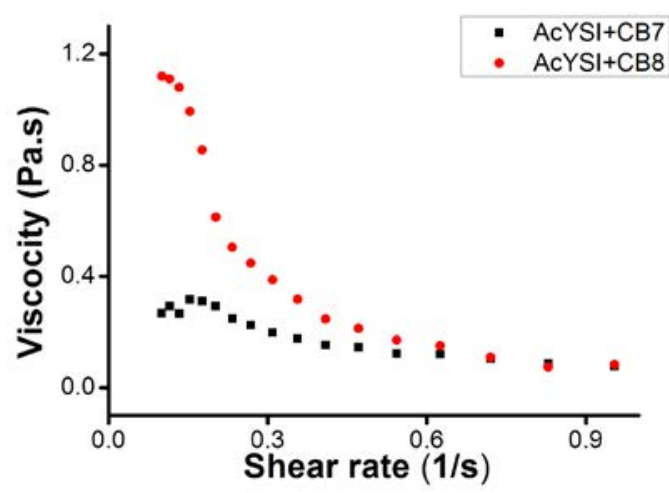

Figure 7.12 - A) Photo graph showing cuvettes containing $3.9 \mathrm{mM}$ of AcYSI with $34 \mu \mathrm{M}$ of either $\mathrm{CB}[7]$ or $\mathrm{CB}[8]$. B) Viscosity versus shear rate profile for AcYSI (3.9 mM) solution containing either $\mathrm{CB}[8]$ or $\mathrm{CB}[7](34 \mu \mathrm{M})$.

\subsection{Conclusions}

In conclusion, our study demonstrates that the self-assembly process of tri- $\beta^{3}$-peptides can be modulated by addition of cucurbit[n]urils. We achieved different phases of chiral assemblies by controlling the lateral interactions of peptide protofibrils. General insight from differentially modulating supramolecular assembly ${ }^{36}$ can lead to new ways to change properties of supramolecular polymers.

\subsection{Acknowledgements}

Dr. Peter Korevaar and Dr. Tom F. A. de Greef are highly acknowledged for their help in modelling of the $\mathrm{CD}$ data and overall discussions.

\subsection{Materials and methods}

General $\beta$-amino acids were purchased from AAPPTec LLC. Wang resin was purchased from Novabiochem. Cucurbit(n)uril (CB[7] and CB[8]) were purchased from Strem chemicals and verified by microcalorimetric titration against paraquat.

Synthesis Peptide synthesis was carried out manually as described previously. ${ }^{28}$ The tri$\beta^{3}$-peptide was synthesized on $0.1 \quad \mathrm{mM}$ scale using standard fluorenylmethyloxycarbonyl (Fmoc) chemistry on Wang resin (1.2 mmol/g loading). A 
typical coupling cycle consists of initially washing of the resin with N-methyl-2pyrrolidone (NMP) (3 x $1 \mathrm{~min}$ ), followed by adding a solution of Fmoc protected $\beta$ amino acids (3,1 eq. with respect to resin loading), hydroxybenzotriazole (HOBT) (3eq.), 2-(1H-benzotriazol-1-yl)-1,1,3,3tetramethyluronium hexafluorophosphate (HBTU) (3eq.) and N,N-diisopropylethylamine (DIPEA) (4.5eq.). 4Dimethylaminopyridine (DMAP) (0.1 eq.) in NMP was added drop wise only during coupling of the first $\beta$-amino acid to the resin. Coupling of the first $\beta$-amino acid was carried out for $12 \mathrm{~h}$ while from the second $\beta$-amino acid onward the coupling was carried out for 2 h. After each coupling cycle the resin was washed ( 3 x $30 \mathrm{sec}$ ) with NMP followed by capping of unreacted $\beta$-amino acids using $10 \% \mathrm{v} / \mathrm{v}$ acetic anhydride and $1 \% \mathrm{v} / \mathrm{v}$ DIPEA in NMP (2 x $20 \mathrm{~min})$. After washing $(3 \times 30 \mathrm{sec}$ ) with NMP, deprotection of the N-terminus of peptide was carried out using $40 \%$ piperidine in NMP (2 x $20 \mathrm{~min}$ ). After washing with NMP (3 x $30 \mathrm{sec}$ ) the same protocol was followed till the last $\beta$-amino acid was coupled to the resin. After the final Fmoc deprotection step, the peptide chain was capped with an acetyl group (by treating the resin with a solution of $10 \% \mathrm{v} / \mathrm{v}$ acetic anhydride and 1\%v/v DIPEA in NMP ( 2 x $20 \mathrm{~min}$ ) and subsequently cleavage of the peptide chain from the resin was achieved by treating resin with a cleavage solution containing $2.5 \% \mathrm{v} / \mathrm{v}$ water and $2.5 \%$ triisopropylsilane in trifluoroacetic acid (TFA) for $90 \mathrm{~min}$. The cleaved resin was washed twice with the cleavage solution ( 2 x $30 \mathrm{sec}$ ) and the cleaved $\beta$-peptide in TFA was collected. The TFA was then evaporated under a stream of $\mathrm{N}_{2}$ and the peptide was precipitated by the addition of diethyl ether. The precipitate was then filtered through a sintered glass funnel and reconstituted in $\mathrm{H}_{2} \mathrm{O}$ /acetonitrile (1:1) for lyophilization. After lyophilization, the crude peptide was purified using preparative high performance liquid chromatography (HPLC) (Waters 2535 quaternary gradient module with XBridge ${ }^{\mathrm{TM}}$ prep C18 $5 \mu \mathrm{m} \mathrm{OPD}{ }^{\mathrm{TM}} 19 \times 250 \mathrm{~mm}$ preparative column). The eluents used were $0.1 \%$ aqueous TFA and $0.1 \%$ TFA in acetonitrile. Extent of purification was checked using an analytical HPLC (Waters 2535 quaternary gradient module XBridge C18 $5 \mu \mathrm{m}$ column with $4.6 \times 250 \mathrm{~mm}$ dimensions). Same eluents as that of preparative HPLC were used. HPLC retention times were observed following analytical HPLC with a solvent gradient of 0-90\% acetonitrile over 90 min (Figure 7.13). Mass of purified peak was determined using LC-MS (Waters 2535 module coupled to micromass LCT)): AcYSI calc. 465.54 [M], found 466.39 [M+H], 488.37 [M+Na] (Figure 7.14). 


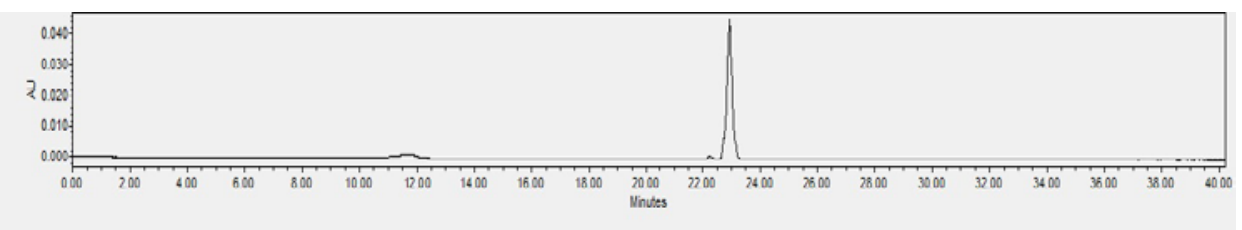

Figure 7.13 - Analytical HPLC trace of AcYSI.

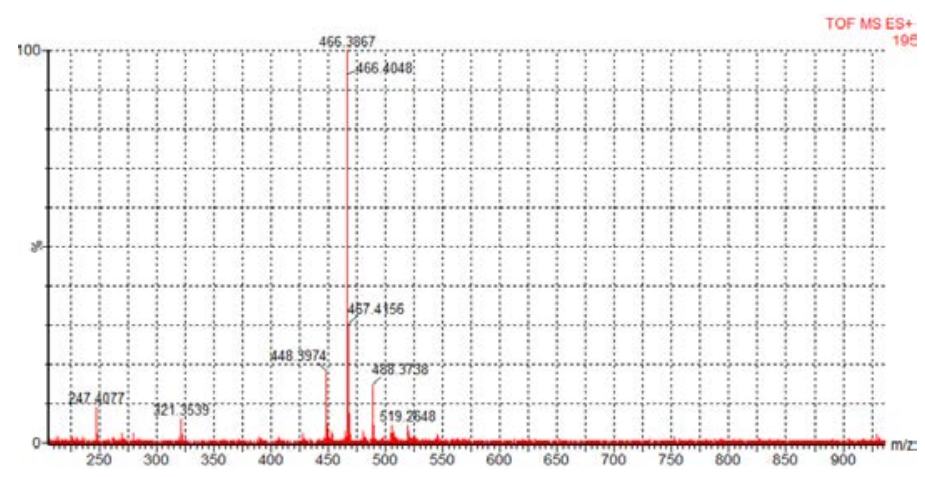

Figure 7.14 - ESI-ToF mass spectrum of AcYSI.

Optical microscopy Olympus CKX41 was used for inspection of samples using optical microscopy. Solutions (in milliQ water) were deposited on clean glass slides using a micropipette and dried carefully.
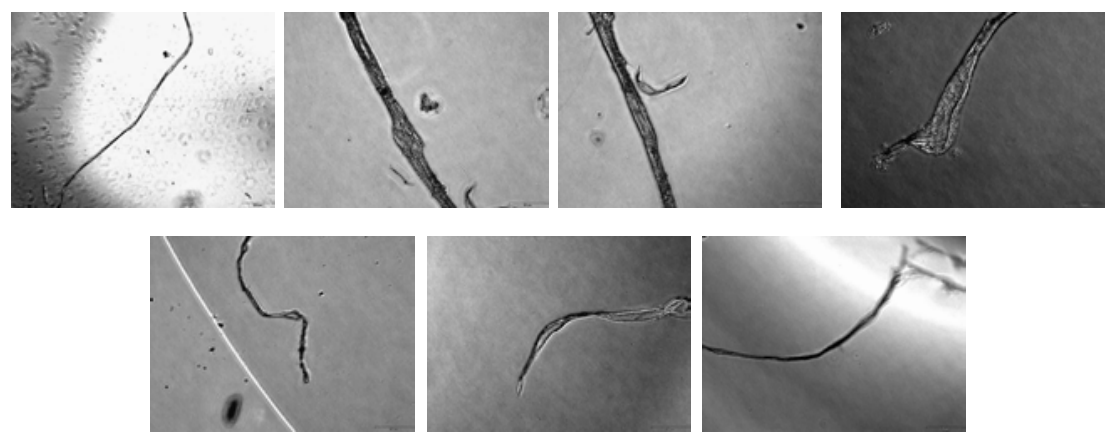

Figure 7.15 - Several optical images of deposits of AcYSI fibrils (3.9 mM). 


\section{SEM microscopy}

Scanning electron microscopy imaging was performed on Carl Zeiss Merlin scanning electron microscope. $2 \mu \mathrm{L}$ of the peptide solution (in milliQ water) was drop cast on copper TEM grids. The solution was dried and analyzed without further treatment of the samples.
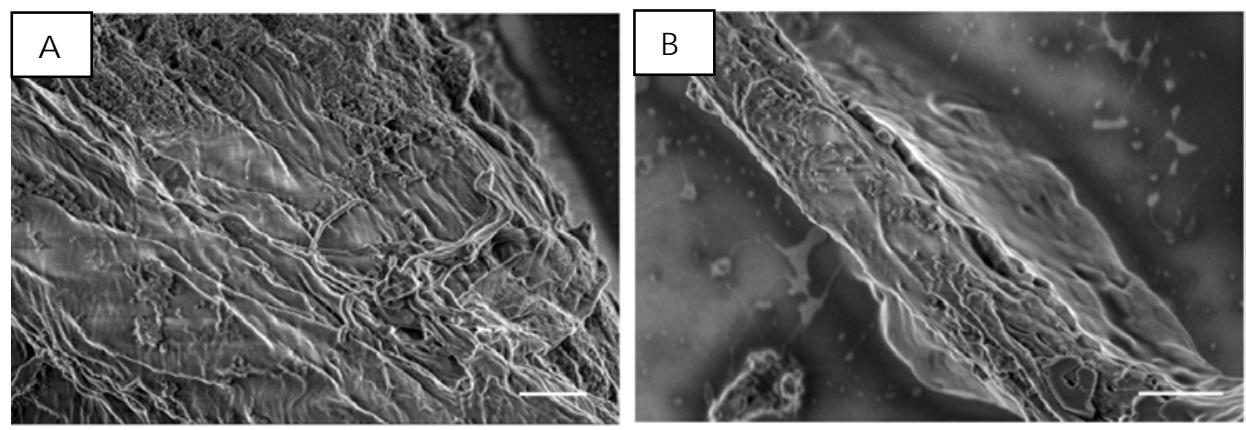

Figure 7.16 - (A) SEM images of AcYSI (3.9 mM) and CB[8] (34 $\mu \mathrm{M})$ and (B) AcYSI alone (3.9 mM). Scale bar $1 \mu \mathrm{m}$.

\section{Viscosity measurements}

Viscosity measurement was done on RHEOPLUS/32 Multi9 V3.10 instrument. 300 ul each of peptide alone, peptide with $\mathrm{CB}[7]$ and $\mathrm{CB}[8]$ containing solution was placed on viscometer and viscosity was measured from shear rate of 0.1 to 100.50 data points were collected for each measurement. Spectra are shown in Figure 7.12.

\section{CD measurements}

All the measurements are done in sterile PBs on Jasco-J1500 instrument. CD scale was 200 mdeg/1.0 Dod, spectra was measured from 195 to 250nm at the scanning speed of $20 \mathrm{~nm} / \mathrm{min}$. Data pitch was set at $0.5 \mathrm{~nm}$ and bandwidth at $1.0 \mathrm{~nm}$. For each measurement two spectra's are measured separately instead of accumulation. In case of temperature interval scan measurement complete spectra's are measured from $90^{\circ} \mathrm{C}$ to $5^{\circ} \mathrm{C}$ with interval of $10^{\circ} \mathrm{C}$ and incubation time ta a particular temperature before scanning was $20 \mathrm{~s}$. with data pitch of $0.1 \mathrm{~nm}$. 

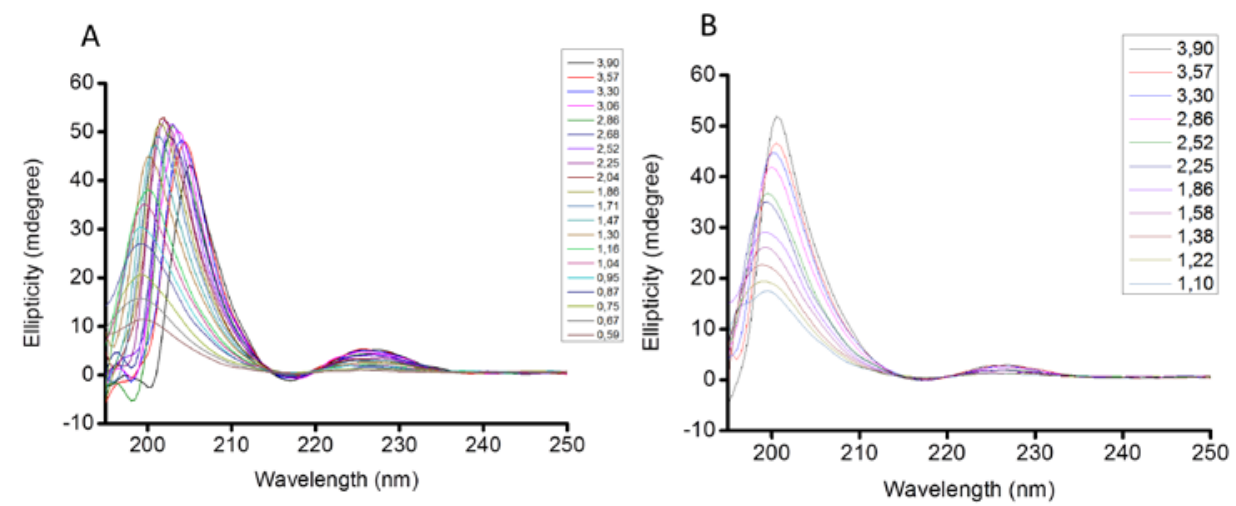

Figure 7.17 - CD spectra of (A) AcYSI (3.9 mM) and CB[7] (34 $\mu \mathrm{M})$ and (B) AcYSI (3.9 $\mathrm{mM})$ with $\mathrm{CB}[8](34 \mu \mathrm{M})$.

\section{The nucleation aggregation model}

The equilibrium model describes the aggregation process as sequential monomer addition equilibria :

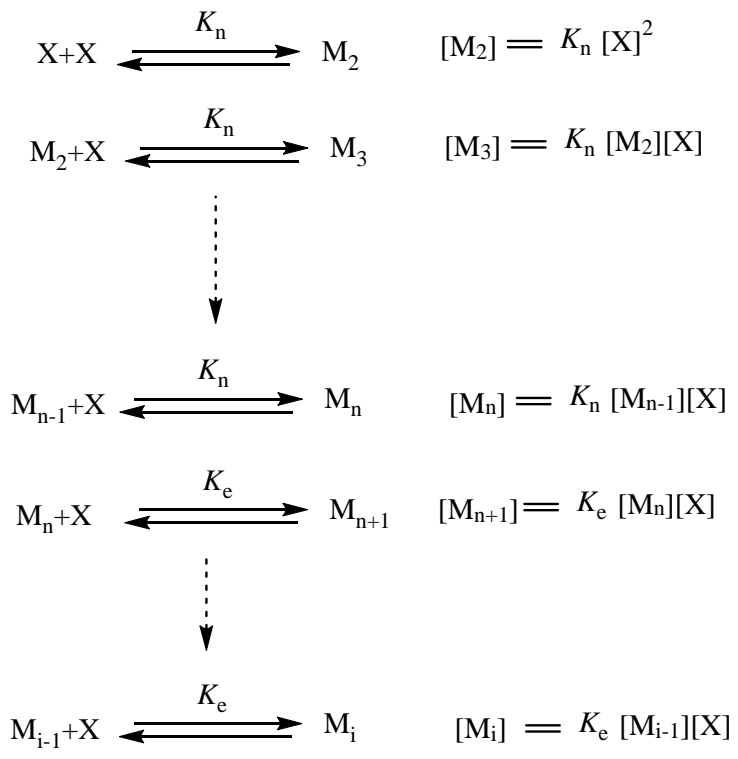

In the above model $\mathrm{X}$ represents the monomer, i.e. the hydrogen bonded dimer of 2 peptide monomers. In case of a co-operative aggregation process, $K_{\mathrm{n}}<K_{\mathrm{e}}$ (with $K_{\mathrm{n}}$ the equilibrium constant of nucleation, $K_{\mathrm{e}}$ the equilibrium constant of elongation and 
co-operativity $\left.\sigma=K_{\mathrm{n}} / K_{\mathrm{e}}\right)$. In an isodesmic aggregation process $K_{\mathrm{n}}=K_{\mathrm{e}}(\sigma=1)$. The concentration of each species $\mathrm{M}_{\mathrm{i}}$ equals $\left[\mathrm{M}_{\mathrm{i}}\right]=K_{\mathrm{n}}{ }^{\mathrm{i}-1}[\mathrm{X}]^{\mathrm{i}}$ for $\mathrm{i} \leq \mathrm{n}$ and $\left[\mathrm{M}_{\mathrm{i}}\right]=K_{\mathrm{e}}{ }^{\mathrm{i}-\mathrm{n}} K_{\mathrm{n}}{ }^{\mathrm{n}-}$ ${ }^{1}[\mathrm{X}]^{\mathrm{i}}$ for $\mathrm{i}>\mathrm{n}$. With dimensionless concentration $\mathrm{m}_{\mathrm{i}}=K_{\mathrm{e}}\left[\mathrm{M}_{\mathrm{i}}\right]$, dimensionless monomer concentration $\mathrm{x}=K_{\mathrm{e}}[\mathrm{X}]$, the dimensionless concentration of each species $\mathrm{M}_{\mathrm{i}}$ equals $\mathrm{m}_{\mathrm{i}}$ $=\sigma^{\mathrm{i}-1} \mathrm{x}^{\mathrm{i}}$ for $\mathrm{i} \leq \mathrm{n}$ and $\mathrm{m}_{\mathrm{i}}=\sigma^{\mathrm{n}-1} \mathrm{x}^{\mathrm{i}}$ for $\mathrm{i}>\mathrm{n}$. Hence dimensionless mass balance is

$$
X_{\text {tot }}=\sigma^{-1} \sum_{i=1}^{n} i(\sigma X)^{i}+\sigma^{n-1} \sum_{i=n+1}^{\infty} i(X)^{i}
$$

with dimensionless total concentration $X_{\text {tot }}=\mathrm{K}_{\mathrm{e}} \cdot \mathrm{C}_{\text {tot }}$ and $\mathrm{C}_{\mathrm{tot}}$ the total monomer concentration in mol/L. Evaluating both sums in eq. 1 using standard expressions for converging series yields :

$$
\begin{array}{r}
X_{t o t}=\sigma^{-1}\left(\frac{(\sigma X)^{n+1}(\mathrm{n} \sigma x-n-1}{(\sigma x-1)^{2}}+\frac{(\sigma X)}{(\sigma x-1)^{2}}\right) \\
-\sigma^{n-1}\left(\frac{(X)^{n+1}(\mathrm{n} x-n-1}{(x-1)^{2}}\right)
\end{array}
$$

Solving equation 2 using standard numerical methods in matlab yields the dimensionless monomer concentration $\mathrm{X}$. Subsequently, if all species with $\mathrm{i}>1$ are considered aggregates, the degree of aggregation can be defined as:

$$
\Phi=\left(X_{t o t}-X\right) / X_{t o t}
$$

After fitting our concentration dependent CD data, assuming initial nucleus size of two. We saw very good fitting suggesting highly co-operative nature of self-assembly. Although it was not possible to reliably extract degree of co-operativity from the fitting but elongation constant $\left(K_{\mathrm{e}}\right)$ was estimated to be $0.0098 \mathrm{mM}^{-1}$. Due to very sharp transition of peptide assembly from monomers to small protofibrills, it was possible to model this regime. At high concentration these individual fibrils comes in close proximity and starts to twist around themselves showing multi step "self-twining" assembly process. But due to very diffused transition of individual fibers to twisted fibers it was not possible to model this regime. 
AFM measurements Atomic force microscopy imaging was performed on AFM Nanoscope III, (Bruker) with intermittent contact mode. $2 \mu \mathrm{L}$ of the peptide solution was applied to freshly cleaved mica surface using glass capillary displacement method. The sample was covered with petri dish to slow down rate of evaporation. After 15 min the sample surface was dried under the stream of $\mathrm{N}_{2}$. Afterwards samples were immediately imaged. Image processing was done using Gwyddion software.

\subsection{References}

1. Wilson, M.R., Yerbury, J.J. \& Poon, S. Potential roles of abundant extracellular chaperones in the control of amyloid formation and toxicity. Molecular BioSystems 4, 42-52 (2008).

2. Aguzzi, A. \& Calella, A.M. Prions: Protein Aggregation and Infectious Diseases. Physiological Reviews 89, 1105-1152 (2009).

3. Auer, S., Dobson, C.M., Vendruscolo, M. \& Maritan, A. Self-Templated Nucleation in Peptide and Protein Aggregation. Physical Review Letters 101, 258101 (2008).

4. Shimizu, T., Masuda, M. \& Minamikawa, H. Supramolecular Nanotube Architectures Based on Amphiphilic Molecules. Chemical Reviews 105, 1401-1444 (2005).

5. O'Leary, L.E.R., Fallas, J.A., Bakota, E.L., Kang, M.K. \& Hartgerink, J.D. Multi-hierarchical self-assembly of a collagen mimetic peptide from triple helix to nanofibre and hydrogel. Nature Chemistry 3, 821-828 (2011).

6. Tidhar, Y., Weissman, H., Wolf, S.G., Gulino, A. \& Rybtchinski, B. Pathway-Dependent Self-Assembly of Perylene Diimide/Peptide Conjugates in Aqueous Medium. Chemistry - A European Journal 17, 6068-6075 (2011).

7. Roche, C. et al. Homochiral Columns Constructed by Chiral Self-Sorting During Supramolecular Helical Organization of Hat-Shaped Molecules. Journal of the American Chemical Society 136, 7169-7185 (2014).

8. Jonkheijm, P., van der Schoot, P., Schenning, A.P.H.J. \& Meijer, E.W. Probing the Solvent-Assisted Nucleation Pathway in Chemical SelfAssembly. Science 313, 80-83 (2006). 
9. Yang, Z., Liang, G., Wang, L. \& Xu, B. Using a Kinase/Phosphatase Switch to Regulate a Supramolecular Hydrogel and Forming the Supramolecular Hydrogel in Vivo. Journal of the American Chemical Society 128, 3038-3043 (2006).

10. Haines, L.A. et al. Light-Activated Hydrogel Formation via the Triggered Folding and Self-Assembly of a Designed Peptide. Journal of the American Chemical Society 127, 17025-17029 (2005).

11. Kang, J. et al. A rational strategy for the realization of chain-growth supramolecular polymerization. Science 347, 646-651 (2015).

12. Korevaar, P.A. et al. Pathway complexity in supramolecular polymerization. Nature 481, 492-496 (2012).

13. Ogi, S., Sugiyasu, K., Manna, S., Samitsu, S. \& Takeuchi, M. Living supramolecular polymerization realized through a biomimetic approach. Nature Chemistry 6, 188-195 (2014).

14. Ogi, S., Stepanenko, V., Sugiyasu, K., Takeuchi, M. \& Würthner, F. Mechanism of Self-Assembly Process and Seeded Supramolecular Polymerization of Perylene Bisimide Organogelator. Journal of the American Chemical Society 137, 3300-3307 (2015).

15. van der Zwaag, D., de Greef, T.F.A. \& Meijer, E.W. Programmable Supramolecular Polymerizations. Angewandte Chemie International Edition 54, 8334-8336 (2015).

16. Song, Y., Cheng, P.-N., Zhu, L., Moore, E.G. \& Moore, J.S. Multivalent Macromolecules Redirect Nucleation-Dependent Fibrillar Assembly into Discrete Nanostructures. Journal of the American Chemical Society 136, 5233-5236 (2014).

17. Chinai, J.M. et al. Molecular Recognition of Insulin by a Synthetic Receptor. Journal of the American Chemical Society 133, 8810-8813 (2011).

18. Heitmann, L.M., Taylor, A.B., Hart, P.J. \& Urbach, A.R. Sequence-Specific Recognition and Cooperative Dimerization of N-Terminal Aromatic Peptides in Aqueous Solution by a Synthetic Host. Journal of the American Chemical Society 128, 12574-12581 (2006). 
19. Logsdon, L.A. \& Urbach, A.R. Sequence-Specific Inhibition of a Nonspecific Protease. Journal of the American Chemical Society 135, 1141411416 (2013).

20. Dang, D.T., Schill, J. \& Brunsveld, L. Cucurbit[8]uril-mediated protein homotetramerization. Chemical Science 3, 2679-2684 (2012).

21. Hou, C. et al. Construction of Protein Nanowires through Cucurbit[8]urilbased Highly Specific Host-Guest Interactions: An Approach to the Assembly of Functional Proteins. Angewandte Chemie International Edition 52, 5590-5593 (2013).

22. Sankaran, S., Kiren, M.C. \& Jonkheijm, P. Incorporating Bacteria as a Living Component in Supramolecular Self-Assembled Monolayers through Dynamic Nanoscale Interactions. ACS Nano 9, 3579-3586 (2015).

23. Lee, H.H. et al. Supramolecular Inhibition of Amyloid Fibrillation by Cucurbit[7]uril. Angewandte Chemie International Edition 53, 7461-7465 (2014).

24. Patgiri, A., Joy, S.T. \& Arora, P.S. Nucleation Effects in Peptide Foldamers. Journal of the American Chemical Society 134, 11495-11502 (2012).

25. Cheng, R.P., Gellman, S.H. \& DeGrado, W.F. $\beta$-Peptides: From Structure to Function. Chemical Reviews 101, 3219-3232 (2001).

26. Pandya, M.J. et al. Sticky-End Assembly of a Designed Peptide Fiber Provides Insight into Protein Fibrillogenesis. Biochemistry 39, 8728-8734 (2000).

27. Ishihara, Y. \& Kimura, S. Four-peptide-nanotube bundle formation by selfassembling of cyclic tetra- $\beta$-peptide using g-quartet motif. Peptide Science 100, 141-147 (2013).

28. Del Borgo, M.P. et al. Supramolecular Self-Assembly of N-Acetyl-Capped $\beta$-Peptides Leads to Nano- to Macroscale Fiber Formation. Angewandte Chemie 125, 8424-8428 (2013).

29. Horne, W.S. \& Gellman, S.H. Foldamers with Heterogeneous Backbones. Accounts of Chemical Research 41, 1399-1408 (2008).

30. Boersma, M.D. et al. Evaluation of Diverse $\alpha / \beta$-Backbone Patterns for Functional $\alpha$-Helix Mimicry: Analogues of the Bim BH3 Domain. Journal of the American Chemical Society 134, 315-323 (2012). 
31. Bautista, A.D., Appelbaum, J.S., Craig, C.J., Michel, J. \& Schepartz, A. Bridged $\beta 3$-Peptide Inhibitors of p53-hDM2 Complexation: Correlation between Affinity and Cell Permeability. Journal of the American Chemical Society 132, 2904-2906 (2010).

32. Hill, S.E., Robinson, J., Matthews, G. \& Muschol, M. Amyloid Protofibrils of Lysozyme Nucleate and Grow Via Oligomer Fusion. Biophysical Journal 96, 3781-3790 (2009).

33. Korevaar, P.A., Newcomb, C.J., Meijer, E.W. \& Stupp, S.I. Pathway Selection in Peptide Amphiphile Assembly. Journal of the American Chemical Society 136, 8540-8543 (2014).

34. Matsuo, K. et al. Characterization of Intermolecular Structure of $\beta 2$ Microglobulin Core Fragments in Amyloid Fibrils by Vacuum-Ultraviolet Circular Dichroism Spectroscopy and Circular Dichroism Theory. Journal of Physical Chemistry B 118, 2785-2795 (2014).

35. Schenning, A.P.H.J., Jonkheijm, P., Peeters, E. \& Meijer, E.W. Hierarchical Order in Supramolecular Assemblies of Hydrogen-Bonded Oligo(pphenylene vinylene)s. Journal of the American Chemical Society 123, 409416 (2001).

36. Kumar, M. et al. A dynamic supramolecular polymer with stimuli-responsive handedness for in situ probing of enzymatic ATP hydrolysis. Nature Communications 5, 1-8 (2014). 


\section{Summary}

Multivalency is the key principle dictating receptor-ligand interactions in biological systems. To understand these interactions in detail a model system taken out of biological complexity is required. Most of the model systems reported so far have been focused on employing strong intrinsic interactions between ligands and receptors. But strong intrinsic interactions in a multivalent system cause large changes in affinity with valency (i.e., cause a high multivalent enhancement factor) and cause the interaction pairs to be in the bound state for most of the time. These properties can lead to a kinetically trapped state of the multivalent complex, which is in contrast to biological systems in which lower multivalency enhancement factors avoid such a kinetically trapped state.

To resolve this issue a weakly multivalent system has been developed in this thesis. The result is a model system with a small multivalency enhancement factor, thus ensuring higher valences not result in a kinetically trapped state of the complex.

We envision that the weakly multivalent model system described in this thesis will help to understand the mechanism of multivalent interactions, for example, the interaction of the influenza virus with a cell, in a biological context. This improved understanding will help to design the next generation of molecular and regenerative medicines. 


\section{Samenvatting}

Multivalentie speelt een sleutelrol in de interacties tussen receptoren en liganden in biologische systemen. Om deze interacties te kunnen begrijpen is een modelsysteem nodig buiten de biologische complexiteit. Het merendeel van de huidige modelsystemen maakt gebruik van sterke intrinsieke interacties tussen liganden en receptoren. Sterke intrinsieke interacties resulteren echter in grote veranderingen in affiniteit bij veranderende valenties (d.w.z ze veroorzaken een hoge multivalente versterkingsfactor). Dientengevolge zijn de interactieparen voor het grootste deel van de tijd in de gebonden toestand. Dit kan een kinetisch ingevroren toestand van het multivalente complex veroorzaken. In biologische systemen echter is de multivalente versterkingsfactor kleiner waardoor deze toestand voorkomen wordt.

Om een oplossing te bieden voor dit probleem is in dit proefschrift een zwak multivalent systeem ontwikkeld. Het resultaat is een modelsysteem met een kleine multivalente versterkingsfactor waardoor hogere valenties niet resulteren in een kinetisch ingevroren complex.

Het zwak multivalente modelsysteem dat in dit proefschrift beschreven is, geeft inzicht in het mechanisme van multivalente interacties in een biologische context, bijvoorbeeld betreffende de interactie tussen een griepvirus en een cel. Dit inzicht zal helpen bij het ontwerp van de volgende generatie moleculaire en regeneratieve therapieën. 


\section{Acknowledgements}

In the summer of 2011, I started a new phase in my scientific career at the Molecular Nanofabrication (MNF) group. It is a great pleasure to express my gratitude for people who helped me during this four years of my doctoral study. Everyone's contribution in shaping of this thesis is indispensable. Firstly, I would like to thank my daily supervisor Prof. Pascal Jonkheijm, for providing me an opportunity to pursue $\mathrm{PhD}$ research under your guidance. It is still one of the happiest moments in my life when I got the offer letter from you. I admire your pleasant personality and logical thinking ability. Your ability to inspire students is phenomenal. Individual meetings have always been a source of motivation for me. Whenever I faced a scientific problem, I could just pass by your office and you were always there for troubleshooting and providing future research directions. Moreover, I really enjoyed the complete freedom provided by you regarding designing of experiments, which helped me to acquire wide range of skills. Next I want to thank my co-promotor Prof. Jurriaan Huskens. You are leading by example. I am highly fascinated with your ability to think fast, clearly and holistically. Every meeting or discussion with you was a great learning experience, particularly during chapter corrections. During social gatherings, I enjoyed your company a lot, especially our discussion about literature and art. Both of you helped me to grow personally and scientifically and I could not wish for a better combination of supervisors than you two. Next, I want to thank Dr. Dorothee Wasserberg (Dodo) and Dr. Qi An (Angel) for your help with the peptide synthesis and SPR techniques during the initial days of my $\mathrm{PhD}$ study. I could always come to you two for any help regarding experimental setups. Next, I want to thank my collaborator Dr. Damien Thompson for your help with MD simulations. Your point of view on host-guest systems gave me new insight into the topic. I am very grateful to you for the possibility to work in your laboratory. I would also like to thank Dr. Tom de Greef and Dr. Peter Korevaar, for their intellectual input and modelling of data in Chapter 7. It is a very intellectually stimulating experience discussing results with you two. Dr. Wim Verboom, Dr. Tibor Kudernac, Prof. Dr. Jeroen Cornelissen, Dr. Nathalie Katsonis, thanks for your feedback on my projects during colloquium presentations. I want to thank Richard, Marcel, Bianca and Regine for your prompt and indispensable help with all the technical problems while 
doing experiments. Nicole and Izabel, thanks for all the help with bureaucratic work, especially during last month’s visa extension issue $:-$. Your kind personality and helpful nature is always heart-warming.

Next, I want to thank my lab mate Tom, you always helped me with any difficulty in setting up instruments. I am a great admirer of your pleasant and cool personality and wish you best of luck with your future endeavours. Supitchaya, a close friend. I really enjoyed our funny conversations and wish you all the best with finishing your thesis. I want to thank my colleagues from lab 4 Sarah, Rik, Martijn, Anne, and Rianne for your help. Rajesh, you are like my elder brother, always helping and guiding me. I really enjoyed our trips and dinners. Chumki, all the best with your $\mathrm{PhD}$ and I wish you two a great life ahead.

I want to thank my paranimfen: Bettina, your pleasant personality and good sense of humour is very refreshing. Moreover, your organization skills are very evolved which was visible from our adjacent computer desks $(;)$. I wish you all the best with your thesis writing. Gülistan, thanks for helping me with the FRAP experiments. I really enjoyed our scientific discussions. I admire your ability to easily fragment complex research questions into smaller logical parts. I wish you all the best with finishing your $\mathrm{PhD}$. Raquel, thanks for your help and all the fun time we spend during thesis writing. Your infectious smile acted as a bolster dose during our monotonous thesis writing. I want to thank Jordi for your help with understanding the complicated SPR setup. Carlo, thanks for your academic as well as non-academic help. I want to thank my group members Joao, Nicole, Maike, Wies and Jens for a great time and wish you best of luck in future endeavours. Jasper, all the best with your start-up. Shirish, thanks for your help with all scientific and non-scientific difficulties. I really enjoyed our scientific discussion especially during social gatherings. Jenny, thanks for your help with the cruel CAM assay (:). Your help with biological experiments was crucial in shaping of Chapter 5. I wish you two a very happy married life and great future ahead. Emanuela, your ability to pay attention to details is incredible. Furthermore your empathy towards colleagues is admirable. Mark, thank you for your help with the Dutch summary. All the best you two with finishing your PhDs.

Shane, Pierre and Melissa, friends from Ireland, thanks for your help during my stay there. I really enjoyed the fun time we spend during lunch and trips. Carmen, Laura, Roberto, I really enjoyed your company during all social gatherings, best of luck with 
your future. I want to thank my current and former colleagues Rick, Luilin, Alexis, Andrea, Daniele, Ye Liang, Alexander, Sven, Pieter, Melanie, Marten, Benjamin, Alejandro, Jealemy, Mudassir, Nicolai, Piotr, Vijay, Rindia, Supaporn, Wilfried, He, Andreas, Janneke, Wouter, Aijie, Christoph, Tetiana, Shyam, Wei, Mark, Rianne, Federico, Raluca for your help. Stan, thanks for your help with the peptide synthesis during your short internship with me. Jijuan and Tegar, thanks for your help with SPR experiments during your bachelor internship with me.

Amal, I really enjoyed your company, especially during lunch time. I will miss our fitness schedule. Wish you the best of luck with your academic career. Finally I would like to thank my best friends, Aditya and Rasika. Aditya, you are always there to give support and advice on taking important descisions in my life. Rasika, I admire your cool personality. I learned important social skills from you and I am sure due to our complementary skill set we will have a great life ahead.

Enschede, August 2015

Tushar Satav 
Tushar Narendra Satav was born in Amravati, India on March 24th 1987. He received a M.S. (Pharm.) degree in medicinal chemistry from National Institute of Pharmaceutical Education and Research (NIPER), India under the supervision of Prof. Dr. Asit K. Chakraborti (FRSC) in July 2011. Towards the fulfillment of M.S. (Pharm.) degree he has submitted a thesis entitled "New scaffolds for selective COX-2 inhibition: Design, synthesis and biological evaluation of novel compounds”. During his M.S. (Pharm.) study, he developed competency in organic chemistry, molecular docking and biological assay techniques.

Since August 2011, he is a PhD under the supervision of Prof. Dr. Ir. Pascal Jonkheijm and Prof. Dr. Ir. Jurriaan Huskens at the University of Twente, where he developed a model system to study weakly multivalent systems. As a step further, he developed a well-defined platform by using this weakly multivalent system for use in new blood vessel development of interest to potentially treat ischemic heart diseases. The results of his research are described in this thesis. 


\section{List of publications}

Patents

1. Asit K. Chakraborti, Dinesh Kumar, Tushar Satav, “An Improved Acid Catalyzed One Pot Synthesis of 2-Styryl Quinoline.” Indian Patent Application No. 2673/DEL/2011 filed on 15.09.2011 (IP17892/VR).

Papers

1. Tushar Satav, Jurriaan Huskens and Pascal Jonkheijm

"Effects of Variations in Ligand Density on Cell Signaling”

Small (2015)

2. Tushar Satav, Qi An, Nicole Georgi, Wies van Roosmalen, Jenny Brinkmann, Dr. Damien Thompson, Jurriaan Huskens and Pascal Jonkheijm

"Supramolecular platforms with programmable multivalency to trigger VEGF-A activity”

Manuscript in preparation

3. Tushar Satav, Gulistan Kocer, Damien Thompson, Jurriaan Huskens and Pascal Jonkheijm

"Surface dynamics of weakly multivalent ligand molecules along a surface functionalized with receptors"

Manuscript in preparation

4. Tushar Satav, Peter Korevaar, Tom de Greef, Jurriaan Huskens and Pascal Jonkheijm

"Modulating the Nucleated Self-Assembly of Tri- $\beta^{3}$-peptides using Cucurbit[n]urils"

Manuscript in preparation 